\title{
Top Quarks at the Tevatron
}

\section{Measurements of the Top Quark Production and Decay with the $D \varnothing$ Experiment}

by

Jonas Strandberg

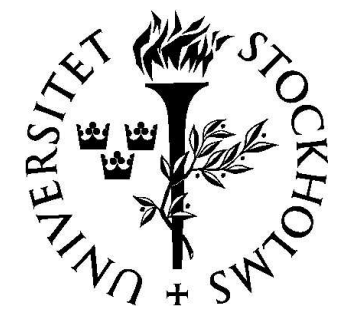

Department of Physics

Stockholm University

2006 
Doctoral Dissertation 2006

Fysikum

Stockholm University

Roslagstullsbacken 21

10691 Stockholm

\begin{abstract}
This thesis presents two measurements of the top quark using $230 \mathrm{pb}^{-1}$ of data recorded with the $\mathrm{D} \varnothing$ detector at the Tevatron accelerator. The first measurement determines the top pair production cross section at $\sqrt{s}=1.96 \mathrm{TeV}$ in proton-antiproton collisions. In the standard model of particle physics the top quark decays almost exclusively into a $W$ boson and a $b$ quark. Candidate events are selected by requiring that at least one jet in the event is tagged with the secondary vertex algorithm. The measured $t \bar{t}$ cross section is:
\end{abstract}

$$
\sigma_{t \bar{t}}=8.6_{-1.5}^{+1.6} \text { (stat. }+ \text { syst.) } \pm 0.6 \text { (lumi.) pb. }
$$

The second measurement uses the observed and predicted number of events with 0,1 and $2 b$-tagged jets to estimate the ratio $R$,

$$
R=\frac{B(t \rightarrow W b)}{B(t \rightarrow W q)}
$$

where $q$ stands for any down-type quark. The measured value is

$$
R=1.03_{-0.17}^{+0.19}(\text { stat }+ \text { syst }),
$$

in good agreement with the standard model prediction of $R \approx 1$. The result is used to set a lower limit on $R$ :

$$
R>0.61 \text { (95\% C.L.). }
$$

(C) Jonas Strandberg 2006

ISBN 91-7155-222-7

Printed by Universitetsservice US AB, Stockholm 2006 


\section{Contents}

1 Introduction 1

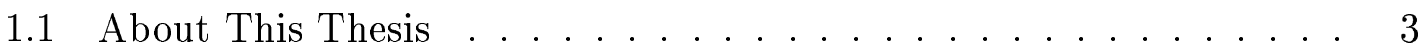

1.2 Author's contributions . . . . . . . . . . . . . 4

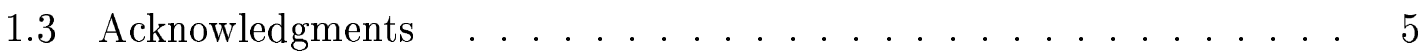

2 Theoretical Background $\quad 7$

2.1 Interactions and Local Gauge Invariance . . . . . . . . . . . 7

2.1.1 The Electro-Weak Interaction ............. 8

2.1.2 The Strong Interaction . . . . . . . . . . . . 10

2.1.3 The Higgs Boson . . . . . . . . . . . . . . . 10

2.2 The Top Quark . . . . . . . . . . . . . . . . . . . 12

2.2.1 Top Quark Production .......................... 12

2.2.2 Top Quark Decay _................. 14

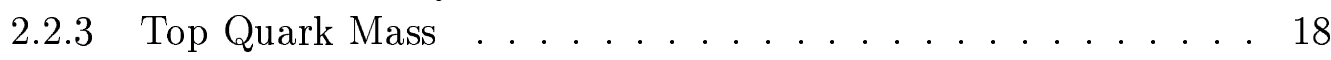

3 The $D \varnothing$ Detector 23

3.1 Charged Particle Identification ............... 24

3.1.1 The Silicon Microstrip Tracker ............ . 25

3.1.2 The Central Fiber Tracker . . . . . . . . . . 28

3.2 Electromagnetic and Hadronic Showers . . . . . . . . . . . . . 29

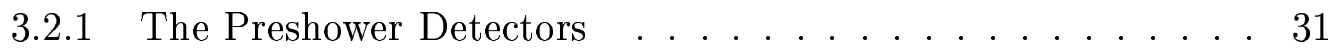

3.2 .2 The Calorimeter . . . . . . . . . . . . 32

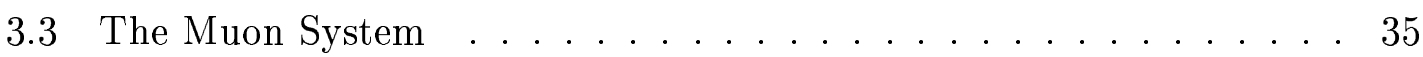

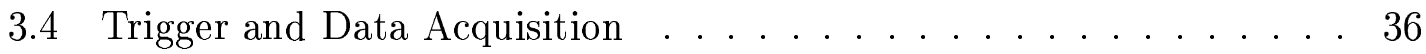

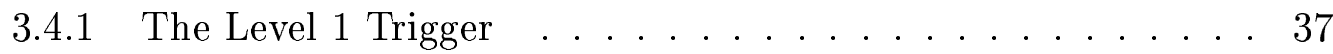

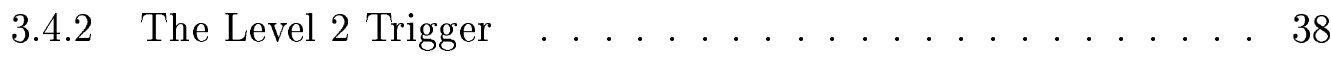

3.4 .3 The Level 3 Trigger . . . . . . . . . . . . . 38

3.5 Luminosity System . . . . . . . . . . . . . . . . 39

4 Object Identification $\quad 41$

4.1 Primary Interaction Point . . . . . . . . . . . . . . 42

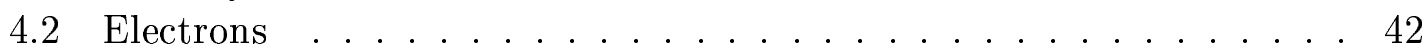




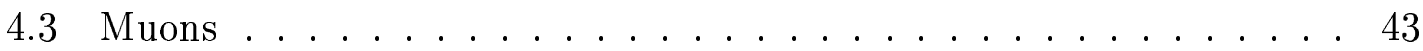

4.4 Jets . . . . . . . . . . . . . . . . . . 45

4.4.1 Track Based Jets ................. . . . . . . . . . . . . . . . 46

4.5 Missing Transverse Energy _.............. . . 47

5 Sample Definitions $\quad 49$

5.1 Data Samples . . . . . . . . . . . . . . . . . 49

5.1.1 The Preselected Signal Sample ... . . . . . . . . 49

5.1 .2 The Muon-in-jet Sample . . . . . . . . . . . . . 50

5.1 .3 The Multijet Data Sample . . . . . . . . . . . 50

5.2 Simulated Samples . . . . . . . . . . . . . . . . 50

5.2 .1 The $t \bar{t}$ Sample . . . . . . . . . . . . . 51

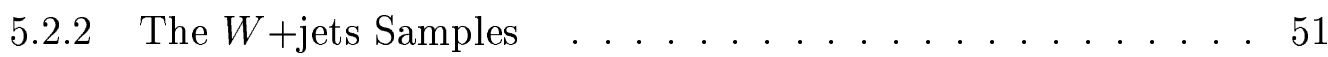

5.2.3 Other Physics Backgrounds ............. 55

6 The Preselected Signal Sample $\quad 59$

6.1 Trigger Selection and Luminosity . . . . . . . . . . . . . 59

6.2 Selection Criteria . . . . . . . . . . . . . 60

6.2.1 Common Selection Criteria for the $e+$ jets and the $\mu+$ jets Channels .................. 61

6.2.2 Selection Criteria Specific to the $e+$ jets Channel ..... . 61

6.2.3 Selection Criteria Specific to the $\mu+$ jets Channel . . . . . . 62

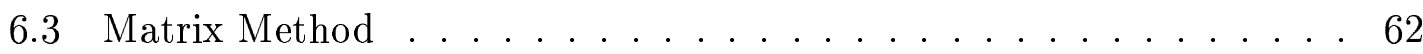

6.4 Sample Composition .................... 63

$\mathbf{7}$ Identification of jets from $b$ quarks $\quad \mathbf{6 9}$

7.1 Secondary Vertex Algorithm . . . . . . . . . . . . 69

7.2 Taggability .......................... 71

7.2.1 Flavor Dependence of Taggability . . . . . . . . . . 73

7.3 Efficiency for Heavy-Flavor Jets . . . . . . . . . . . . . 75

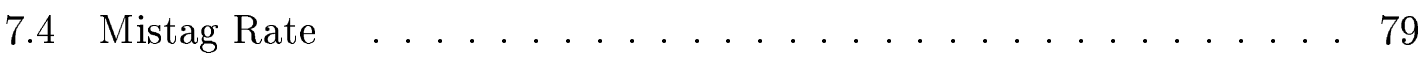

7.4.1 Correction Factors . . . . . . . . . . . . 81

8 The $b$-tagged Signal Sample $\quad 87$

8.1 Event Tagging Probability . . . . . . . . . . . . . . 87

8.1.1 The Multijet Background . . . . . . . . . . . 88

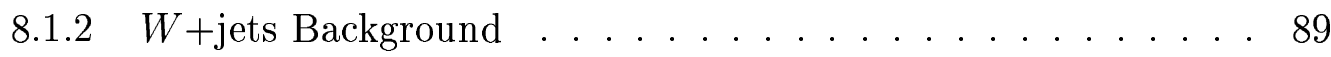

8.1.3 Other Physics Backgrounds . . . . . . . . . . 90

8.1 .4 The $t \bar{t}$ Signal . . . . . . . . . . . . . . . . . 90

8.2 Final Sample Composition . . . . . . . . . . . . . . 90

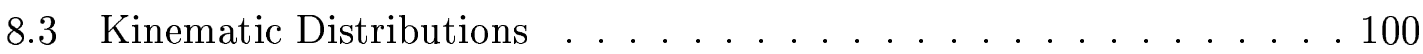


9 Results

9.1 Measurement of $\sigma_{t \bar{t}} \ldots \ldots \ldots \ldots 111$

9.1.1 Systematic Uncertainties . . . . . . . . . . . 114

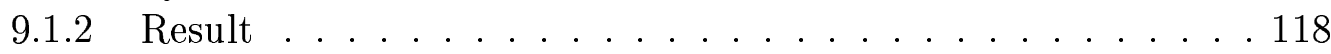

9.2 Simultaneous Measurement of $\sigma_{t \bar{t}}$ and $R \ldots \ldots . \ldots . \ldots 120$

9.2.1 $R$-dependence of Event Tagging Probabilities for $t \bar{t}$ Events . . 122

9.2.2 Number of $t \bar{t}$ Events in the Sample Without b-tags . . . . . 124

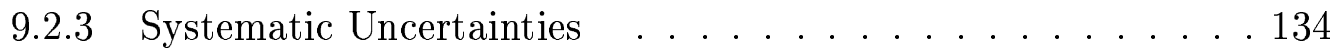

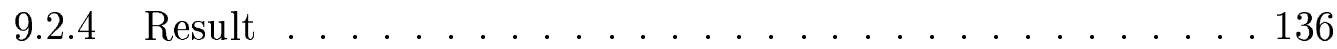

10 Conclusions and Outlook 141 



\section{Chapter 1}

\section{Introduction}

Particle physics is the study of the fundamental constituents of matter and their interactions. During the 20th century remarkable progress was made in the understanding of physics at the microscopic scale. The theories describing the interactions between elementary particles were summarized into the standard model of particle physics (SM). The SM describes the strong interaction between quarks and gluons, called Quantum Chromo Dynamics (QCD) [1, 2, 3], and the unified theory of electromagnetic and weak interactions called the electro-weak theory $[4,5,6]$. The electro-weak theory and QCD are quantum field theories based on the principle of local gauge invariance $[7,8]$. All known elementary particles and their properties are included in the standard model. The particles are divided into fermions with half-integral spin and bosons with integral spin. The building blocks of matter are fermions whereas the force carriers which allow particles to interact with each other are bosons.

The SM has successfully predicted many experimental results to an extraordinary precision [9]. One central aspect of the theory is however still unconfirmed by experiments. The electro-weak symmetry is not an exact symmetry at low energies. In the SM the electro-weak symmetry is broken by postulating the existence of a new complex scalar field, called the Higgs field $[10,11,12]$. As a consequence of the Higgs field there must exist a new massive spin-0 particle called the Higgs boson. The detection of the Higgs boson has been one of the major goals of experimental high energy physics since its prediction 40 years ago.

Despite its predictive success, the standard model cannot be a complete theory of everything. The most obvious reason is that it fails to incorporate gravity and thus breaks down at energies of the order of the Planck scale $\left(m_{\mathrm{P}} \sim 10^{18} \mathrm{GeV} / c^{2}\right)$. The recent discovery that neutrinos oscillate between the different flavor states $[13,14]$ implies that they have a non-zero mass which is not predicted by the standard model. Furthermore, the SM does not provide a satisfactory answer to the origin of the matter versus anti-matter asymmetry in nature [15].

Large particle colliders have been built to test the predictions of the SM and 


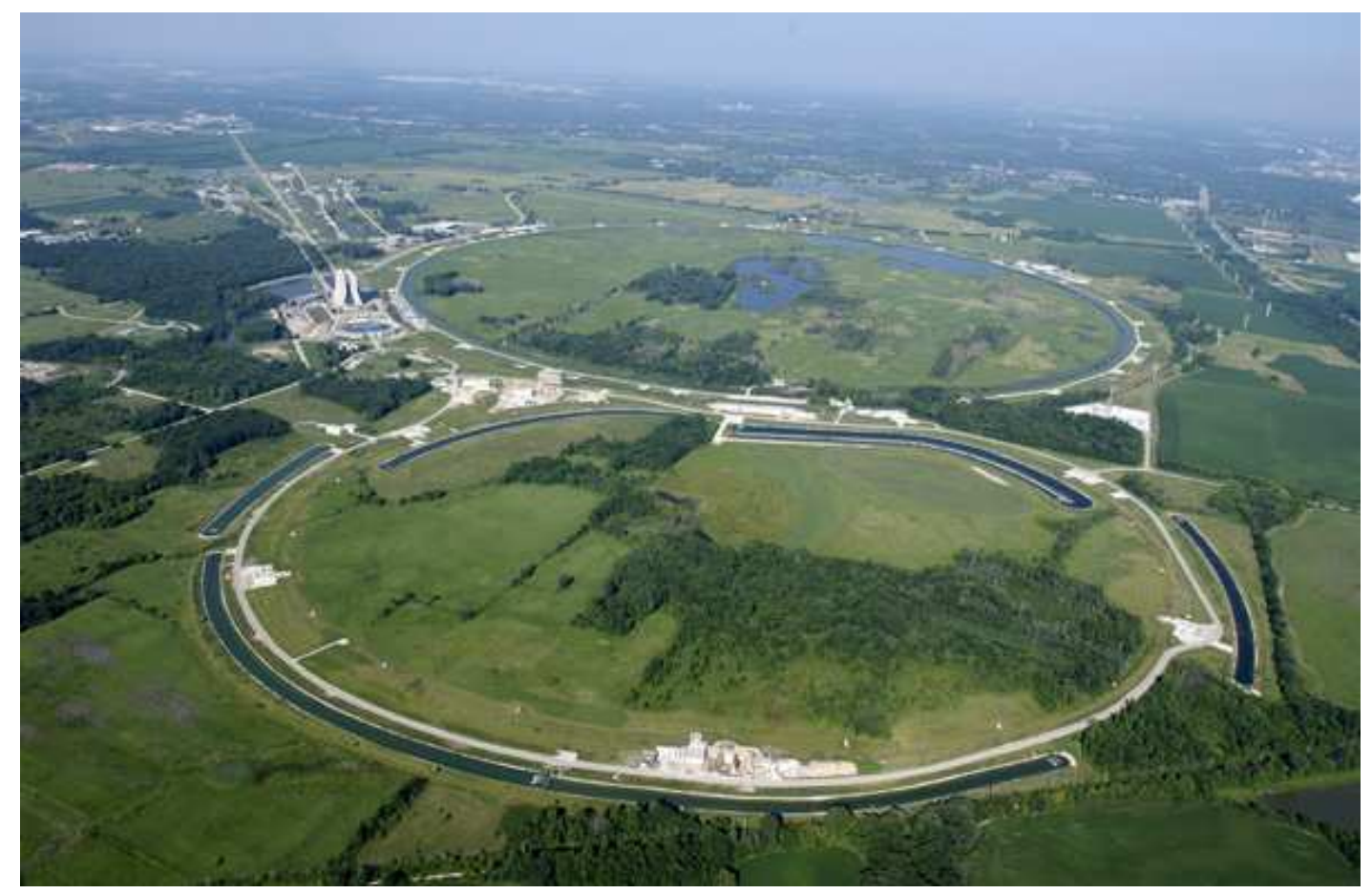

Figure 1.1: An aerial view of Fermilab with the Main Injector in the foreground and the Tevatron accelerator in the background.

to search for new physics. Beams of particles are accelerated to high energies and brought together to collide at interaction points. Surrounding the interaction points particle detectors are built to record and analyze the results of the collisions.

The Tevatron collider located at Fermilab near Chicago is the highest energy collider currently operating in the world. It collides beams of protons and antiprotons at a center-of-mass energy of $1.96 \mathrm{TeV}$. The Tevatron has a radius of $1 \mathrm{~km}$ and the protons and anti-protons are circulated around the ring in a magnetic field of 4.2 T. An aerial view of Fermilab is shown in Fig. 1.1.

$\mathrm{D} \varnothing$ and $\mathrm{CDF}$ are the two experiments recording the collisions at the Tevatron. The first data taking period, called Run I, lasted from 1992 to 1996. During this period the Tevatron operated at the center-of-mass energy of $1.8 \mathrm{TeV}$. Many interesting physics results were obtained in Run I, the most important being the long awaited discovery of the top quark in 1995 by both D $\varnothing$ and CDF [16, 17]. Starting in 1996, the Tevatron accelerator underwent major upgrades aimed mainly at increasing the instantaneous luminosity [18]. The two experiments, DØ and CDF, were also upgraded to take full advantage of the increased collision rate [19, 20]. Data taking was resumed in March 2001, which marked the start of the Run II of the Tevatron.

The Tevatron is still the only accelerator built with sufficient center-of-mass 
energy to produce top quark pairs. The increase in luminosity and center-of-mass energy has led to a much larger sample of top quark events in Run II compared to Run I. The properties of the top quark are important parameters in the standard model, and in all theories trying to extend the standard model to include new physics. Due to its large mass ${ }^{1}$ the top quark must couple strongly to the Higgs boson. Together with electro-weak precision measurements [9], the value of the top quark mass constrains the allowed mass range for the Higgs boson. The large mass of the top quark could also provide hints for the origin of mass and be a probe for physics beyond the standard model.

\subsection{About This Thesis}

This thesis is based on two measurements in the $t \bar{t} \rightarrow \ell+$ jets final state. The measurements are presented in the following papers, referred to in the text by their Roman numerals:

I DØ Collaboration

"Measurement of the $t \bar{t}$ Production Cross Section in $p \bar{p}$ Collisions at $\sqrt{s}=1.96$ TeV using Lepton + Jets Events with Lifetime b-tagging"

Phys. Lett. B 626 (2005) 35.

II D Collaboration

"Simultaneous Measurement of $B(t \rightarrow W b) / B(t \rightarrow W q)$ and the $t \bar{t}$ Production Cross Section at $\sqrt{s}=1.96 \mathrm{TeV}$ "

Approved by the DØ Collaboration for submission to Phys. Rev. Lett.

The thesis is organized as follows. Chapter 2 gives an overview of the standard model and discusses in more detail the role of the top quark. Chapter 3 describes the $\mathrm{D} \varnothing$ detector. The identification of important physics objects is described in Chapter 4 . Chapter 5 defines the different samples needed in the analysis. Chapter 6 describes the sample of candidate events after the first selections, based on event kinematics, have been applied. Chapter 7 gives a more in-depth description of the identification of jets originating from $b$ quarks. The identification of $b$-quark jets is called $b$-tagging, and plays a crucial role in the two measurements in Papers I and II. Chapter 8 describes the final sample of candidate events after the $b$-tagging algorithm has been applied. Finally, Chapter 9 describes how the number of observed events after all selections is translated into the two measurements in Papers I and II. Chapter 10 summarizes the results and compares them to similar measurements performed by the $\mathrm{D} \varnothing$ and CDF collaborations.

\footnotetext{
${ }^{1}$ The top quark is 35 times larger than the second heaviest quark, the $b$ quark.
} 


\subsection{Author's contributions}

This thesis presents the result of my research work at Fermilab during the years 2000 to 2005. In the summer of 2000, I started working with the production of the D $\varnothing$ Silicon Microstrip Tracker (SMT) [21]. At this time the assembly of silicon modules was at its peak. My task was to diagnose and repair malfunctioning modules.

At the end of 2000 the detector was fully assembled and ready to be installed in the collision hall. Starting in February of 2001 I contributed to the cabling and commissioning of the SMT. In March the same year beams were circulated in the Tevatron for the first time in Run II. At this point only a quarter of the SMT was cabled, but the Tevatron beam was very unstable in this early stage of Run II and there were many week-long shutdowns. At the end of May, the SMT was fully cabled and the work was shifted to commissioning of the detector.

I started my work in the top group in the autumn of 2002. Due to the very large size of the $D \varnothing$ data sample, it is not feasible for all users to analyze every event. Therefore the data needs to be split into smaller samples more suitable for analysis. In $\mathrm{D} \varnothing$ this procedure is called skimming, and my first assignment in the top group was to do skimming of offline events into smaller physics samples. This task later became centralized within the $\mathrm{D} \varnothing$ experiment.

I am one of the developers of the top group analysis code. The code resides in a software package called top_analyze. I was responsible for the implementation and maintenance of the $b$-tagging and primary vertex code.

In January of 2003 I became involved in the analysis of the $t \bar{t}$ production cross section using $b$-tagging. It was the first analysis of the $t \bar{t}$ cross section in D $\varnothing$ that uses $b$-tagging. A significant part of the work went into studying the performance of the secondary vertex algorithm. I have been responsible for deriving parameterizations for $b$-tagging efficiency and mistag rates. This is described further in Chapter 7.

During 2003 to 2004 I worked mostly on the analysis presented in Paper I. This analysis has been done in several iterations with increasing size of the data sample. The result has been communicated at the several conferences.

In 2004 I started working on an extension of the analysis in Paper I. The measured fractions of $t \bar{t}$ events with 0,1 and $2 b$-tagged jets were used to perform a simultaneous measurement of the $t \bar{t}$ cross section and the ratio $R=B(t \rightarrow W b) / B(t \rightarrow W q)$, where $q$ is any down-type quark. The result of this measurement is presented in Paper II. 


\subsection{Acknowledgments}

To the members of the $D \varnothing$ Collaboration for all the hard work you put into running the experiment.

To my advisor Barbro Åsman for getting me into particle physics. For all the time spent at Fermilab. For teasing me about basketball. For always trusting me, believing in me and for making these five years totally worth it.

To my second advisor Christophe Clément for your brains and your good sense of humor. For throwing parties and making us forget about work. For reminding us about work. For half of my publications.

To Mom and Dad for raising me, loving me and always being proud of me.

To my sisters. For the fights and the fun.

To Aurelio Juste, for all the physics you taught me. For all the physics you made me do. For inspiring me.

To Lisa Shabalina, Flera Rizatdinova and Sasha Khanov for being my $b$-tagging gurus.

To Markus Klute, Marc-Andre Pleier and Tobias Golling for setting the standard.

To Nils Gollub, Yann Coadou and Per Hansson for great laughs, long nights and lively discussions.

To everyone in the particle physics group in Stockholm.

To my love, Sara. You mean the universe to me.

Thank you all! 



\section{Chapter 2}

\section{Theoretical Background}

The standard model describes all the known elementary particles as well as three of the four fundamental forces of nature. The gravitational force is very weak at distances and energies currently available in particle physics experiments and will be so for the foreseeable future. Although the large scale behavior of gravity is accurately described by the theory of general relativity very little is known about the quantum nature of the force. It is therefore omitted from the standard model.

The birth of the standard model was the unification of the weak and the electromagnetic forces by Glashow, Weinberg and Salam in $1968[4,5,6]$. Another milestone in the development of the SM was the formulation of strong force in terms of Quantum Chromo Dynamics (QCD) [1, 2, 3] in the 1970's.

The matter particles in the SM are spin- $1 / 2$ fermions. They are divided into six leptons and six quarks. The leptons and quarks are grouped into three generations. Each generation contains one electron-like and one neutrino-like lepton plus an uptype and a down-type quark. The forces are mediated by spin- 1 bosons. The exchange particle for the electromagnetic force is the photon $\gamma$. The weak force is mediated by the $W^{ \pm}$and $Z$ bosons and the strong force is mediated by the eight gluons $g$. All particles in the SM are summarized in Table 2.1.

\subsection{Interactions and Local Gauge Invariance}

The exact form of the interactions between particles in the SM can be derived by postulating the invariance of the Lagrangian under local gauge transformations. To ensure invariance, new terms have to be introduced in the Lagrangian. The new terms correspond to interactions between the matter particles, and can be shown to reproduce the correct equations of motion. The unified weak and electromagnetic force is described by the $\mathrm{SU}(2) \times \mathrm{U}(1)$ symmetry group while the strong force is described by the $\mathrm{SU}(3)$ group. The forces are described in more detail in Secs. 2.1.1 and 2.1.2 below. 


\begin{tabular}{lccc}
\hline \hline & \multicolumn{3}{c}{ Generation } \\
& $\mathrm{I}$ & $\mathrm{II}$ & $\mathrm{III}$ \\
\hline Leptons & $\nu_{e}(1953)$ & $\nu_{\mu}(1962)$ & $\nu_{\tau}(2000)$ \\
& $e(1897)$ & $\mu(1936)$ & $\tau(1975)$ \\
\hline Quarks & $u(1968)$ & $c(1974)$ & $t(1995)$ \\
& $d(1968)$ & $s(1968)$ & $b(1977)$ \\
\hline Gauge bosons & \multicolumn{3}{c}{$W^{ \pm}, Z(1900)$} \\
& \multicolumn{3}{c}{$g_{1}, \ldots, g_{8}(1979)$} \\
& $H($ Not discovered) \\
\hline \hline
\end{tabular}

Table 2.1: The elementary particles in the standard model and their year of discovery. The Higgs boson $H$ has not been discovered yet.

\subsubsection{The Electro-Weak Interaction}

The gauge principle associated with the electromagnetic interaction is the invariance of the Lagrangian under local phase rotations. The exact form of the interaction between electrically charged fermions and the mediator of the electromagnetic interaction, the photon, can be derived from this invariance.

The form of the weak interaction is derived from the invariance under gauge transformations in weak isospin space. This leads to interactions where a charged boson $W^{ \pm}$or a neutral boson $W^{0}$ is exchanged. The charged states correspond to the observed $W^{ \pm}$bosons and they lead to charged current interactions in agreement with observations. However the $W^{0}$ boson implies a neutral current process with the same strength as the charged current process. This is not in agreement with observations. The solution to the problem with the neutral current weak interactions lies in the unification of the electromagnetic and the weak force. By requiring invariance under the combined $\mathrm{SU}(2)_{L} \times \mathrm{U}(1)_{Y}$ symmetry group, and introducing another neutral boson $B^{0}$ as the gauge boson for the $\mathrm{U}(1)_{Y}$ group, one can define the photon and the $Z$ boson as linear combinations of the $B^{0}$ and $W^{0}$ bosons,

$$
\begin{aligned}
& \gamma=B^{0} \cos \theta_{W}+W^{0} \sin \theta_{W} \\
& Z=-B^{0} \sin \theta_{W}+W^{0} \cos \theta_{W}
\end{aligned}
$$

where $\theta_{W}$ is the weak mixing angle. It can be shown that the unification condition

$$
e=g_{W} \sin \theta_{W}=g_{Z} \cos \theta_{W}
$$

is such that the $\gamma$ in equation 2.1 has precisely the properties of the observed photon.

After electro-weak unification one problem still remains. In any unbroken gauge field theory, the gauge bosons are required to be massless. Explicit mass terms 
for the bosons in the Lagrangian are not invariant under gauge transformations. However both the $W^{ \pm}$and $Z$ bosons are heavy particles according to experiments. This problem is solved through the mechanism of spontaneous symmetry breaking, which is described in Sec. 2.1.3.

The weak interaction is maximally parity violating and couples only to lefthanded fermions. Experiments have shown that the charged weak currents only mediate transitions within each generation of leptons, coupling with the same strength to charged leptons and neutrinos. Flavor changing neutral currents have never been observed which excludes transitions between generations by $Z$ bosons.

The properties of the weak force are incorporated in the SM by the introduction of weak isospin. Every particle is assigned a weak isospin $I$ and a value for the projection of the third component $I_{3}$. The left handed leptons and quarks are part of an $I=1 / 2$ isospin doublet while right-handed quarks and charged leptons are $I=0$ isospin singlets, see Table 2.2. The $W^{ \pm}$bosons are assigned an isospin value of 1 which allow them to mediate transitions between the left-handed leptons and quarks. The $Z$ boson is an isospin 0 particle, and therefore does not mediate transitions between different flavors. This ordering of the particles neatly expresses the invariance of the weak force under $\mathrm{SU}(2)_{L}$ rotations in the weak isospin space.

\begin{tabular}{|c|c|c|c|c|c|c|}
\hline & \multicolumn{3}{|c|}{ Leptons } & $I_{3}$ & $\mathrm{Y}$ & Q \\
\hline doublet & $\left.\begin{array}{c}\nu_{e} \\
e\end{array}\right)$ & $\begin{array}{c}\nu_{\mu} \\
\mu\end{array}$ & $\left.\begin{array}{c}\nu_{\tau} \\
\tau\end{array}\right)$ & $\begin{array}{r}1 / 2 \\
-1 / 2\end{array}$ & $\begin{array}{l}-1 \\
-1\end{array}$ & $\begin{array}{r}0 \\
-1\end{array}$ \\
\hline singlet & & & & & & \\
\hline singlet & $e_{R}$ & $\mu_{R}$ & $\tau_{R}$ & 0 & -2 & -1 \\
\hline doublet & $\left.\begin{array}{l}u \\
d^{\prime}\end{array}\right)$ & $\begin{array}{l}c \\
s^{\prime}\end{array}$ & & $\begin{array}{r}1 / 2 \\
-1 / 2\end{array}$ & $\begin{array}{l}1 / 3 \\
1 / 3\end{array}$ & $\begin{array}{r}2 / 3 \\
-1 / 3\end{array}$ \\
\hline singlet & $u_{R}$ & $\overline{c_{R}}$ & $t_{R}$ & 0 & $4 / 3$ & $2 / 3$ \\
\hline singlet & $d_{R}^{\prime}$ & $s_{R}^{\prime}$ & $b_{R}^{\prime}$ & 0 & $-2 / 3$ & $-1 / 3$ \\
\hline
\end{tabular}

Table 2.2: The electro-weak eigenstates of the matter particles in the standard model together with their value of the weak isospin $I_{3}$, their hypercharge $Y$ and their electric charge $Q=I_{3}+Y / 2$.

If the weak eigenstates listed in Table 2.2 were also the mass eigenstates, weak transitions would only be allowed within each generation. This is true for the charged leptons. The same was thought to be true for the neutrinos until recently when neutrino oscillations were observed $[13,14]$. For the quarks, the mass eigenstates differ from the weak eigenstates which allows the weak interaction to mediate transitions between the different generations. The weak eigenstates of the down-type quarks $\left(d^{\prime}, s^{\prime}, b^{\prime}\right)$ can be written as superpositions of the three down-type quark mass eigen- 
states $(d, s, b)$ with the coefficients given by the $3 \times 3$ CKM matrix:

$$
\left(\begin{array}{c}
d^{\prime} \\
s^{\prime} \\
b^{\prime}
\end{array}\right)=\left(\begin{array}{lll}
V_{u d} & V_{u s} & V_{u b} \\
V_{c d} & V_{c s} & V_{c b} \\
V_{t d} & V_{t s} & V_{t b}
\end{array}\right)_{C K M}\left(\begin{array}{c}
d \\
s \\
b
\end{array}\right)
$$

The matrix elements $V_{i j}$ are proportional to the coupling at the quark- $W$ vertex, describing the transition from quark $i$ to $j$. Since the neutrinos have been observed to have non-zero mass a similar matrix must also exist for the mixing in the neutrino sector.

\subsubsection{The Strong Interaction}

The complicated spectrum of mesons and baryons was successfully described in the 1960's as being bound states of quarks. To avoid violation of the Pauli principle, a new quantum number called color had to be introduced.

The strong interaction between quarks and gluons is derived from local gauge invariance under $\mathrm{SU}(3)$ rotations in color space. Local invariance can only be achieved by introducing 8 new gauge fields into the Lagrangian, corresponding to the eight gluons which mediate the strong force. The gluons themselves also carry color charge, giving rise to gluon self-interactions.

All hadrons are postulated to be color singlets. Mesons consist of a quarkantiquark pair in a color-anticolor state. Baryons consist of three quarks, with each quark carrying a different color to make the baryon colorless. Besides the two or three valence quarks, there exists a sea of virtual quarks and gluons which also contribute to the total energy and momentum of the hadron.

\subsubsection{The Higgs Boson}

The non-zero mass of the weak gauge bosons implies that the local SU $(2)_{L} \times \mathrm{U}(1)_{Y}$ symmetry of the electro-weak Lagrangian is not an exact symmetry. However the Lagrangian should still be invariant under $\mathrm{U}(1)_{\mathrm{EM}}$ transformations, since the photon is observed to be massless.

The only known way of accomplishing the task of breaking $\mathrm{SU}(2)_{L} \times \mathrm{U}(1)_{Y}$ down to $\mathrm{U}(1)_{\mathrm{EM}}$ while maintaining the initial gauge invariance of the Lagrangian is by the formalism of spontaneous symmetry breaking. A symmetry is said to be spontaneously broken if the theory's Lagrangian is invariant under this symmetry but its vacuum state is not.

In the Higgs mechanism $[10,11,12]$ the spontaneous symmetry breaking is induced by a new electro-weak doublet of complex scalar fields. These fields interact through a potential with an assumed form as illustrated in Fig. 2.1. The key feature of this potential is that at least one of the components of the complex Higgs fields must be non-zero for it to reach its minimum. 


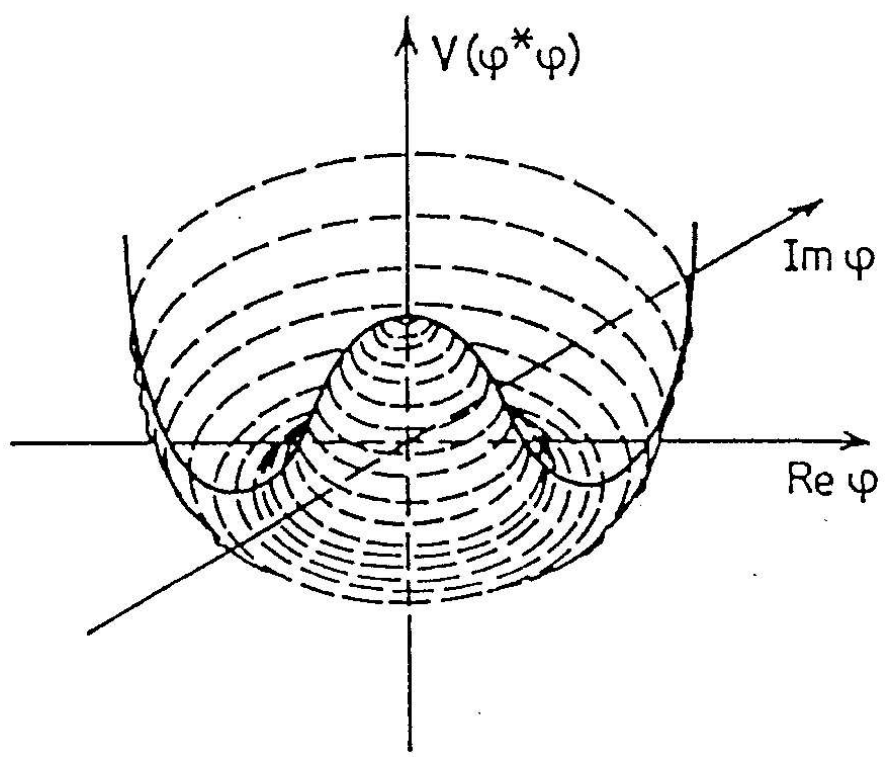

Figure 2.1: The Higgs potential as a function of two of its four degrees of freedom.

To predict the particle spectrum of the theory, the Lagrangian is evaluated in the vicinity of a specific vacuum state. In order to obtain an electrically neutral vacuum, the non-zero component is chosen to be along the real axis of the neutral Higgs field. The process of selecting a specific vacuum state breaks the initial $\mathrm{SU}(2)_{L} \times \mathrm{U}(1)_{Y}$ symmetry, and the real component of the neutral Higgs field acquires a non-vanishing vacuum expectation value $v$. When expanding the Lagrangian around its vacuum state, effective mass terms for the electro-weak gauge bosons $W^{ \pm}$and $Z$ appear. Three of the initial four degrees of freedom of the Higgs fields are transformed into the longitudinal components of the weak gauge bosons $W^{ \pm}$and $Z$. The remaining degree of freedom gives rise to a new physical state, the Higgs boson, with charge and spin 0 .

In the standard model, the masses of the weak gauge bosons are related to the vacuum expectation value of the Higgs field $v$ through:

$$
\begin{aligned}
m_{W} & =\frac{g}{2} v \\
m_{Z} & =\frac{g}{2 \cos \theta_{W}} v=\frac{m_{W}}{\cos \theta_{W}}
\end{aligned}
$$

The $\rho_{0}$-parameter, defined as

$$
\rho_{0}=\frac{m_{W}^{2}}{m_{Z}^{2} \cos ^{2} \theta_{W}}
$$

is predicted to be unity which is in very good agreement with the observed value [22]. 
This result represents one of the great achievements of the SM and imposes strict limits on possible new theories or extensions of the SM.

The vacuum expectation value of the Higgs field can be determined to be:

$$
v \approx 246 \mathrm{GeV} .
$$

However, the mass of the Higgs boson $\left(m_{H}\right)$ remains a free parameter of the model and must be determined experimentally. The search for the Higgs boson has become one of the most pressing topics in high energy physics. To date, no Higgs boson has been observed. The most stringent direct search limit comes from the combined LEP experiments. They exclude a SM Higgs boson with a lower bound on its mass of $m_{H}=114.4 \mathrm{GeV} / c^{2}$ at the $95 \%$ confidence level (CL) [23].

\subsection{The Top Quark}

The top quark was predicted since the discovery of the $b$ quark in 1977, and the discovery of the top quark in 1995 completed the three generation structure of the Standard Model.

The top quark is the heaviest known elementary particle with a mass of approximately $175 \mathrm{GeV} / c^{2}$. The Tevatron, with a center-of-mass energy of $\sqrt{s}=1.96 \mathrm{TeV}$, is at present the only collider where top quarks can be produced and studied. The measurements of the production rate and the properties of the top quark represent important tests of the standard model.

\subsubsection{Top Quark Production}

The top quark can be produced either in pairs via the strong force or singly via the weak force. To date, top quarks have only been observed in the pair production mode. The Feynman diagrams for the leading order pair production processes are shown in Fig. 2.2.

The $t \bar{t}$ cross section increases with the center-of-mass energy, as shown in Fig. 2.3. The relative importance of the $g g \rightarrow t \bar{t}$ processes also increase with the center-ofmass energy. The partons (quarks or gluons) which take part in the hard scatter process carry only a fraction $x$ of the energy of the incoming proton or anti-proton. At higher center-of-mass energies the minimum value of $x$ needed to produce a $t \bar{t}$ pair is lower. Since the gluon density increases rapidly at small values of $x$ the processes involving gluons in the initial state increase in significance at high energies. Figure 2.4 shows the probability to find a certain type of quark or gluon as a function of $x$. This is called a parton density function (PDF). The vertical lines in the plot indicate the minimum values of $x$ needed to produce a $t \bar{t}$ pair, assuming both incoming partons in the hard scatter process carry the same momentum fraction. The dominant pair production mode for $t \bar{t}$ events at the Tevatron energy is the $q \bar{q} \rightarrow t \bar{t}$ process, contributing to $85 \%$ of the total cross section. The $g g \rightarrow t \bar{t}$ process 

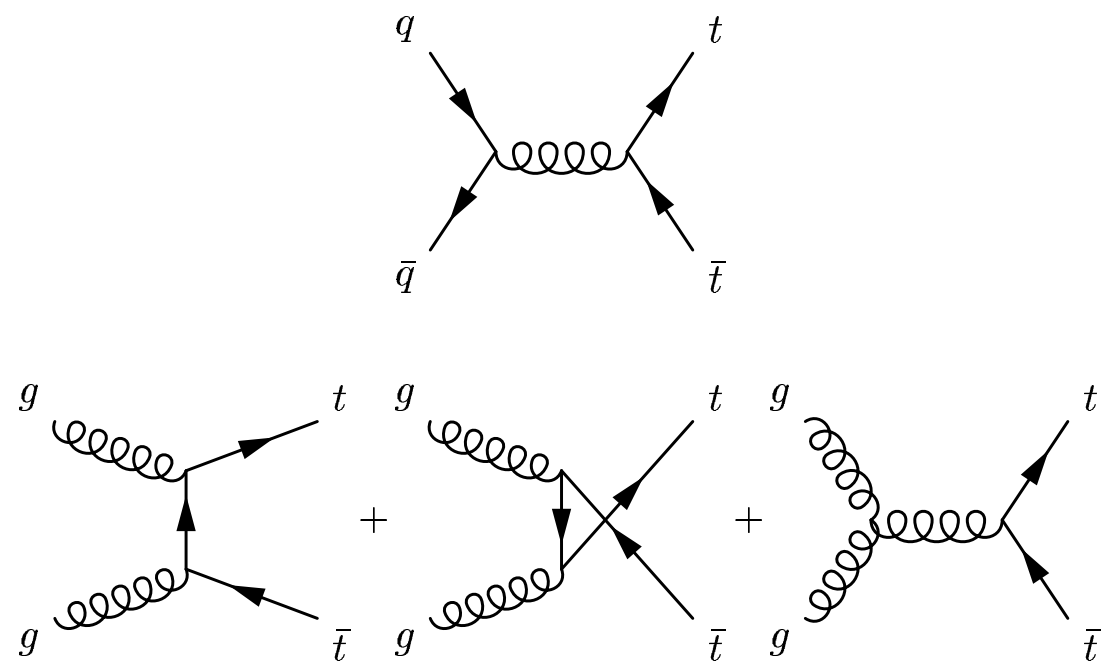

Figure 2.2: Lowest order Feynman diagrams for the production of $t \bar{t}$ pairs at the Tevatron.

contributes only $15 \%$ at the Tevatron but will become the dominant production mode at the LHC. The theoretical $t \bar{t}$ cross section and the contributions from quarkantiquark annihilation and gluon fusion are summarized in Tab. 2.3.

\begin{tabular}{lcccc}
\hline & $\sigma_{t \bar{t}}[26,27]$ & $\sigma_{t \bar{t}}[28,29,30]$ & $q \bar{q} \rightarrow t \bar{t}$ & $g g \rightarrow t \bar{t}$ \\
\hline Tevatron Run I $(\sqrt{s}=1.8 \mathrm{TeV}, p \bar{p})$ & $5.19_{-0.68}^{+0.52} \mathrm{pb}$ & $5.24 \pm 0.31 \mathrm{pb}$ & $90 \%$ & $10 \%$ \\
Tevatron Run II $(\sqrt{s}=1.96 \mathrm{TeV}, p \bar{p})$ & $6.70_{-0.88}^{+0.71} \mathrm{pb}$ & $6.77 \pm 0.42$ & $85 \%$ & $15 \%$ \\
LHC $(\sqrt{s}=14.0 \mathrm{TeV}, p p)$ & $833_{-39}^{+52} \mathrm{pb}$ & $872.8_{-27.6}^{+2.3} \mathrm{pb}$ & $10 \%$ & $90 \%$ \\
\hline
\end{tabular}

Table 2.3: The theoretical $t \bar{t}$ cross sections for the Tevatron Run I and Run II and the LHC. The first column of $t \bar{t}$ cross sections are taken from Ref. [26], with updates from Ref. [27]. The second column of $t \bar{t}$ cross sections are taken from Refs. [28, 29]. A more detailed discussion on the systematic uncertainties can be found in Ref. [30].

The top quark mass dependence of the $t \bar{t}$ cross section is shown in Fig. 2.5 [28]. For a constant center-of-mass energy of $1.96 \mathrm{TeV}$, the cross section decreases with increasing top quark mass.

\section{Single Top Quark Production}

So far, only top quark pair production has been observed in experiments. Single top quarks can be produced via the weak interaction. Some examples of leading order Feynman diagrams for single top production are shown in Fig. 2.6. The expected production cross section is $0.88 \pm 0.07 \mathrm{pb}$ in the s-channel and $1.98 \pm 0.21 \mathrm{pb}$ in 


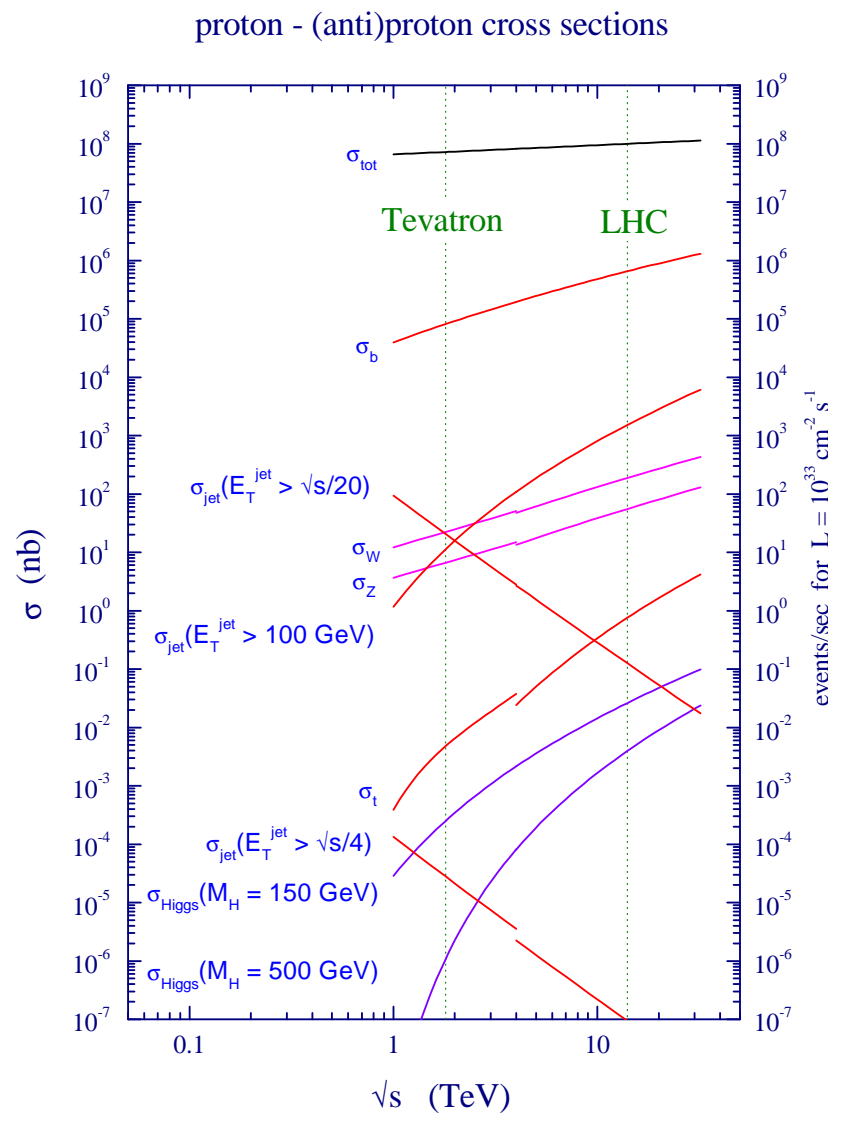

Figure 2.3: QCD predictions for hard scattering cross sections at the Tevatron and the LHC [24]. The $t \bar{t}$ cross section is labeled $\sigma_{t}$ in this plot.

the t-channel $[31,32,33,34,35]$. The theoretical cross section for single top quark production is comparable to the $t \bar{t}$ cross section. However the final state for single top production contains fewer jets which leads to a higher level of background.

\subsubsection{Top Quark Decay}

The top quark has a very short lifetime of approximately $5 \times 10^{-25} \mathrm{~s}$, corresponding to a decay width of $\Gamma_{t} \approx 1.5 \mathrm{GeV} / c^{2}[36]$. The lifetime is one order of magnitude shorter than the characteristic time scale of the strong interaction. The top quark will therefore decay before it can hadronize and form bound states.

The top quark decays into a $W$ boson and a down-type quark. The flavor of the down-type quark is determined by the CKM matrix. Since the matrix element $V_{t b}$ is constrained in the SM to be

$$
0.9990<\left|V_{t b}\right|<0.9992
$$

the top quark decays almost exclusively into a $W$ boson and a $b$ quark [22]. Paper II 


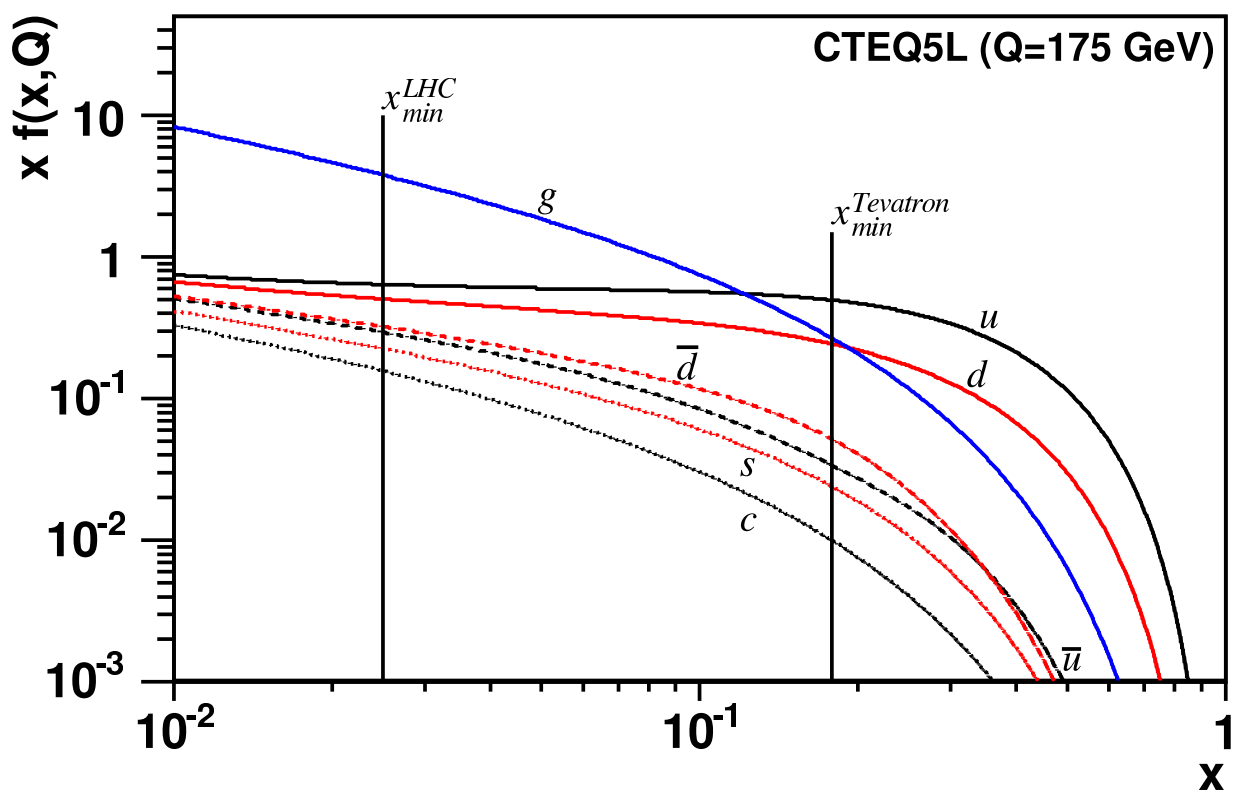

Figure 2.4: Probability to find a certain type of quark or gluon as a function of the momentum fraction $x$ of the proton, given by the CTEQ5L [25] parameterizations. The minimum values of $x$ needed to produce a $t \bar{t}$ pair at the LHC and at the Tevatron are indicated by the vertical bands, assuming both incoming partons carry the same momentum fraction.

presents a measurement of the quantity:

$$
R=\frac{\left|V_{t b}\right|^{2}}{\left|V_{t b}\right|^{2}+\left|V_{t s}\right|^{2}+\left|V_{t d}\right|^{2}} .
$$

In the SM the ratio $R$ is equal to $\left|V_{t b}\right|^{2} \approx 1$. A deviation from the expected value of $R$ implies sources of new physics. The existence of a fourth quark generation would imply a non-unitary $3 \times 3$ CKM matrix. Non-standard model particles in the selected $t \bar{t}$ sample or in the decay of the top quark could also influence the measured value of $R$.

The final state of the $t \bar{t} \rightarrow W^{+} b W^{-} \bar{b}$ process depends on the decays of the two $W$ bosons. A $W$ boson can decay to a charged lepton and a neutrino or to a $q \bar{q}^{\prime}$ pair. All three lepton generations $(e, \mu, \tau)$ are kinematically allowed. The hadronic $W$ decay modes are kinematically limited to the production of first or second generation $q \bar{q}^{\prime}$ pairs. All three leptonic $W$ decay modes have the same probability if higher order corrections are neglected. The two hadronic modes are three times as likely to occur as a leptonic decay due to the color factor of three. Altogether there are nine potential decay modes, all with the same probability of $1 / 9$ at leading order. Due to higher order corrections this symmetry between the decay modes is slightly 


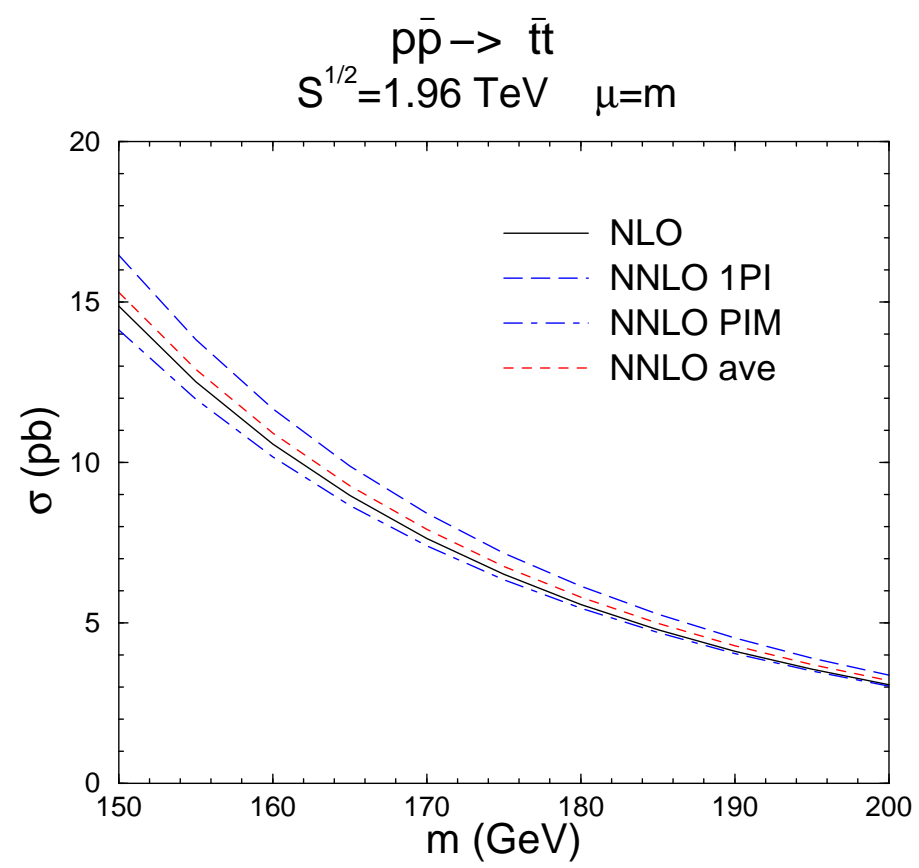

Figure 2.5: The top quark mass dependence of the $t \bar{t}$ cross section. The exact definition of the terms which are considered in the perturbative expansion referred to as "NNLO" can be found in [28].

broken. A summary of the $W$ decay modes is shown in Tab. 2.4.

\begin{tabular}{|c|c|c|c|}
\hline \multicolumn{3}{|c|}{ Decay mode } & $B R[22]$ \\
\hline$\overline{W^{+}}$ & $\rightarrow$ & $e^{+} \nu_{e}$ & $(10.75 \pm 0.13) \%$ \\
\hline$W^{+}$ & $\rightarrow$ & $\mu^{+} \nu_{\mu}$ & $(10.57 \pm 0.15) \%$ \\
\hline$W^{+}$ & $\rightarrow$ & $\tau^{+} \nu_{\tau}$ & $(11.25 \pm 0.20) \%$ \\
\hline$W^{+}$ & $\rightarrow$ & $u \bar{d}, c \bar{s}$ & $(67.60 \pm 0.27) \%$ \\
\hline
\end{tabular}

Table 2.4: Best known branching fractions for the decay of a real $W^{+}$boson [22]. The branching fractions for the charge conjugated processes are identical.

The possible final states of $t \bar{t}$ events are categorized according to the decays of the two $W$ bosons:

Dilepton: Both $W$ bosons decay leptonically. The signature is two charged leptons, two neutrinos and two $b$ quarks, i.e. $\ell \nu \ell^{\prime} \nu^{\prime}+2$ jets.

Lepton plus jets: One $W$ boson decays leptonically and one hadronically. The signature is one charged lepton, one neutrino, a $q \bar{q}^{\prime}$ pair and two $b$ quarks, i.e. $\ell \nu+4$ jets. 

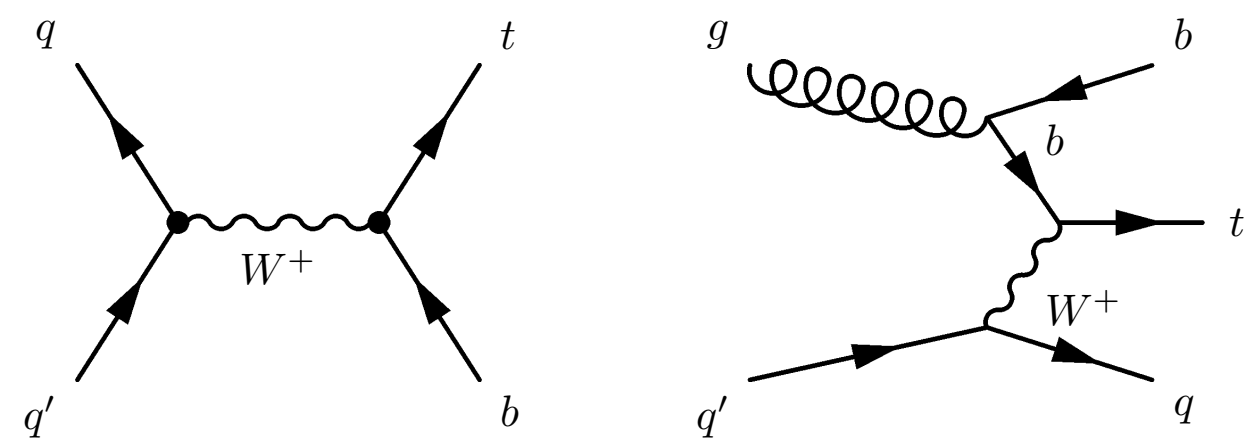

Figure 2.6: Examples of single top quark production via the weak interaction. The main contributions at the Tevatron are from the s-channel process (left) and the t-channel process (right).

All hadronic: Both $W$ bosons decay hadronically. The signature is two $q \bar{q}^{\prime}$ pairs and two $b$ quarks, i.e. 6 jets.

The resulting final states for $t \bar{t}$ events are shown in Fig. 2.7. The all hadronic channel has the largest branching fraction but suffers from a very large multijet background. Final states including $\tau$ leptons are also experimentally challenging. Therefore it is custom to only consider final states containing electrons or muons. This will include a subsample of the $\tau$ final states when the $\tau$ decays leptonically $\tau \rightarrow \nu_{\tau} \nu_{\ell} \ell$ with $\ell=\mu, e$. Table 2.5 gives the relevant branching fractions for each signature.

\begin{tabular}{ccccc}
\hline Channel & \multicolumn{3}{c}{ Decay mode } & $B R[22]$ \\
\hline & $t \bar{t}$ & $\rightarrow$ & $e^{+} \nu_{e} e^{-} \bar{\nu}_{e} b b$ & $(1.14 \pm 0.02) \%$ \\
& $t \bar{t}$ & $\rightarrow$ & $e^{+} \nu_{e} \mu^{-} \bar{\nu}_{\mu} b \bar{b}$ & $(2.28 \pm 0.04) \%$ \\
& $t \bar{t}$ & $\rightarrow$ & $\mu^{+} \nu_{\mu} \mu^{-} \bar{\nu}_{\mu} b \bar{b}$ & $(1.14 \pm 0.02) \%$ \\
$e e$ & $t \bar{t}$ & $\rightarrow$ & $e_{(\tau)}^{+} e_{(\tau)}^{-} b \bar{b}+\nu^{\prime} s$ & $(1.58 \pm 0.03) \%$ \\
$\mu \mu$ & $t \bar{t}$ & $\rightarrow$ & $e_{(\tau)}^{+} \mu_{(\tau)}^{-} b \bar{b}+\nu^{\prime} s$ & $(3.16 \pm 0.06) \%$ \\
$e \mu$ & $t \bar{t}$ & $\rightarrow$ & $\mu_{(\tau)}^{+} \mu_{(\tau)}^{-} b \bar{b}+\nu^{\prime} s$ & $(1.57 \pm 0.03) \%$ \\
\hline & $t \bar{t}$ & $\rightarrow$ & $e^{+} \nu_{e} q \bar{q}^{\prime} b b$ & $(14.52 \pm 0.09) \%$ \\
& $t \bar{t}$ & $\rightarrow$ & $\mu^{+} \nu_{\mu} q \bar{q}^{\prime} b \bar{b}$ & $(14.52 \pm 0.09) \%$ \\
$e+$ jets & $t \bar{t}$ & $\rightarrow$ & $e_{(\tau)}^{+} q \bar{q}^{\prime} b \bar{b}+\nu^{\prime} s$ & $(17.11 \pm 0.11) \%$ \\
$\mu+$ jets & $t \bar{t}$ & $\rightarrow$ & $\mu_{(\tau)}^{+} q \bar{q}^{\prime} b \bar{b}+\nu^{\prime} s$ & $(17.04 \pm 0.11) \%$ \\
\hline all hadronic & $t \bar{t}$ & $\rightarrow$ & $q \bar{q}^{\prime} q \bar{q}^{\prime} b b$ & $(46.19 \pm 0.46) \%$ \\
\hline & $t \bar{t}$ & $\rightarrow$ & $\tau$ final states & $(20.21 \pm 0.13) \%$ \\
\hline
\end{tabular}

Table 2.5: The $t \bar{t}$ decay channels and the best known branching fractions [22]. The charge conjugated final states are implied. $\ell_{(\tau)}$, with $\ell=(e, \mu)$, include both the decay modes $W \rightarrow \ell \nu$ and $W \rightarrow \tau \nu \rightarrow \ell+\nu \nu \nu$. The final states considered in $\mathrm{D} \varnothing$ measurements are indicated in the column labeled "Channel". 


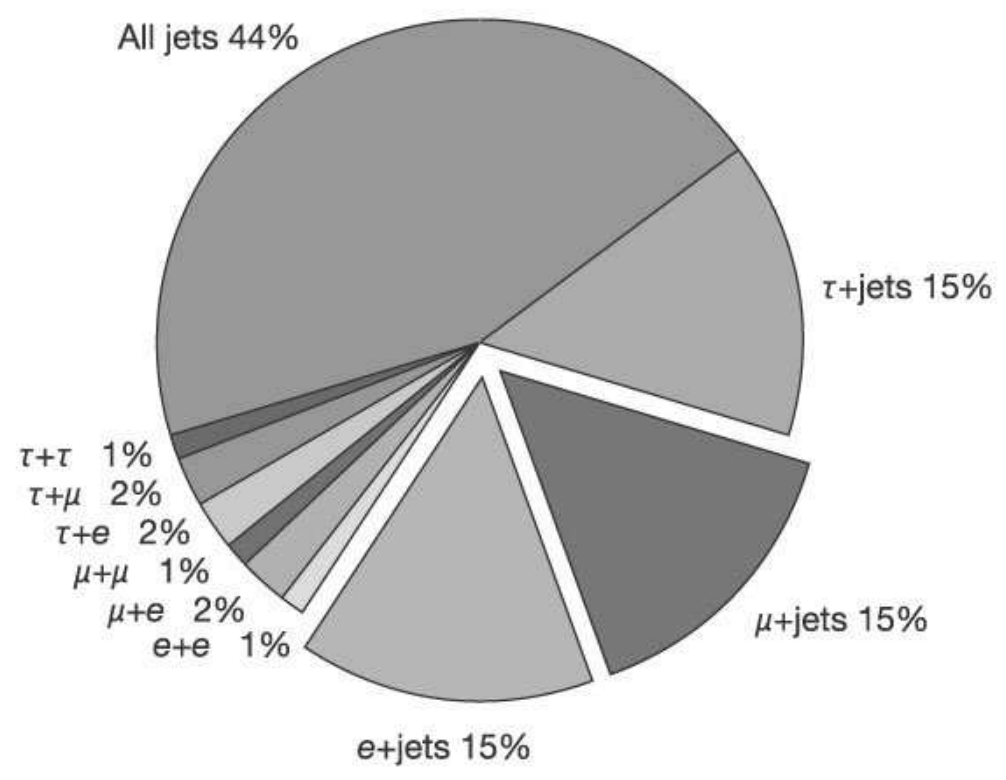

Figure 2.7: The final states of $t \bar{t}$ events are classified according to the decays of the two $W$ bosons.

\subsubsection{Top Quark Mass}

The top quark is heavier than any other elementary particle and its mass is measured with better relative precision than any other quark, as shown in Fig. 2.8. The Tevatron experiments have measured the top quark mass to be [37]:

$$
m_{t}=172.7 \pm 2.9 \text { (stat. }+ \text { syst.) } \mathrm{GeV} / c^{2} \text {. }
$$

This result is the combination of the published Run I measurements $[38,39,40]$ and the preliminary Run II results [41, 42, 43, 44, 45, 46, 47].

The top quark mass is a very important parameter in the standard model, and in all theories trying to extend the SM to include new physics. To generate such a large mass for the top quark the coupling to the Higgs boson must be $\sim 1$. This means that the mass of the Higgs boson strongly depends on the mass of the top quark through loop corrections. An example of a loop correction involving top quarks is shown in Fig. 2.9(a). Radiative corrections also influence the mass of the $W$ boson. The two most important corrections come from loops involving top quarks and loops involving Higgs bosons, shown in Fig. 2.9(b). The most likely value for the mass of the Higgs boson can be deduced from a global fit to all precision electroweak observables [9]. The constraint on the mass of the Higgs boson is shown in Fig. 2.10(a). Figure 2.10(b) shows the constraint on the Higgs boson mass in the $\left(m_{t}, m_{W}\right)$-plane.

The perspective for the full Run II data set is to measure the top quark mass 


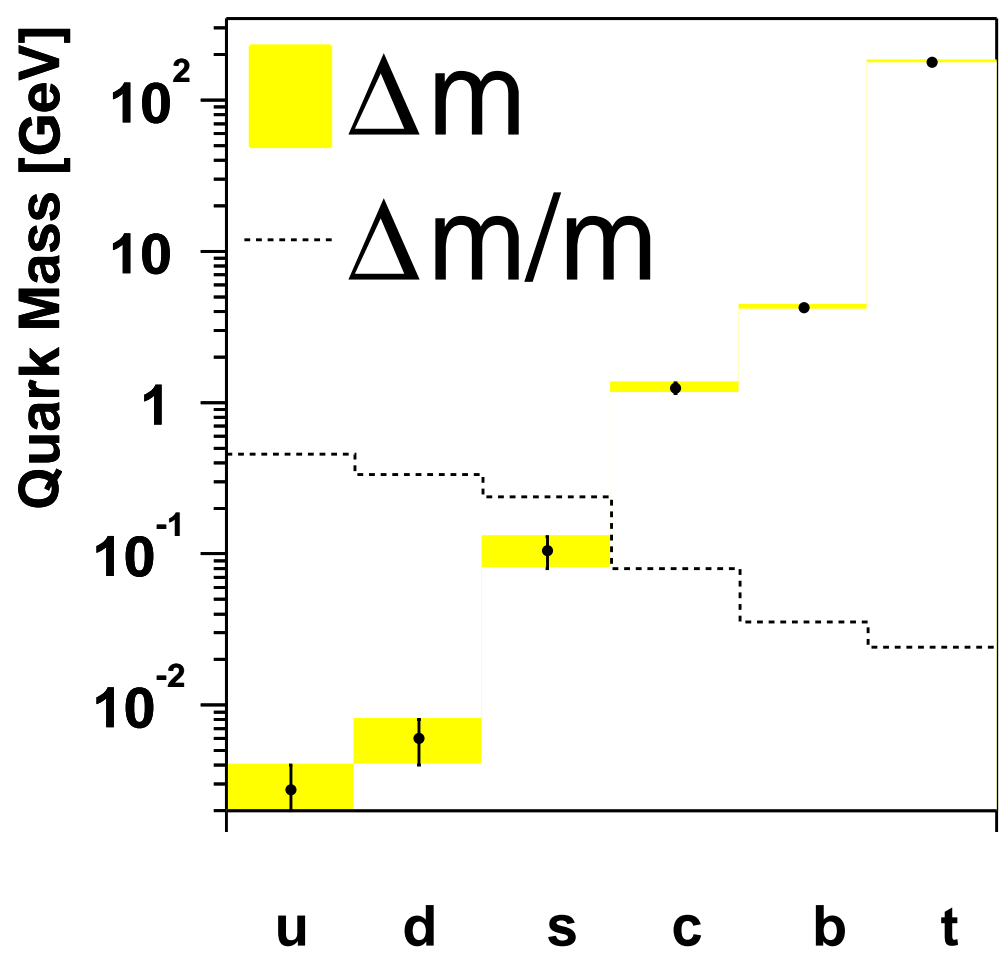

Figure 2.8: The quark masses and their uncertainties.

to a precision better than $3 \mathrm{GeV} / c^{2}$ per experiment. With an uncertainty on the $W$ mass of $20 \mathrm{MeV} / c^{2}$, the Higgs mass is expected to be constrained to better than $40 \%$ [48]. At the LHC the mass of the top quark will be measured with a precision of approximately $1 \mathrm{GeV} / c^{2}[49,50]$. 


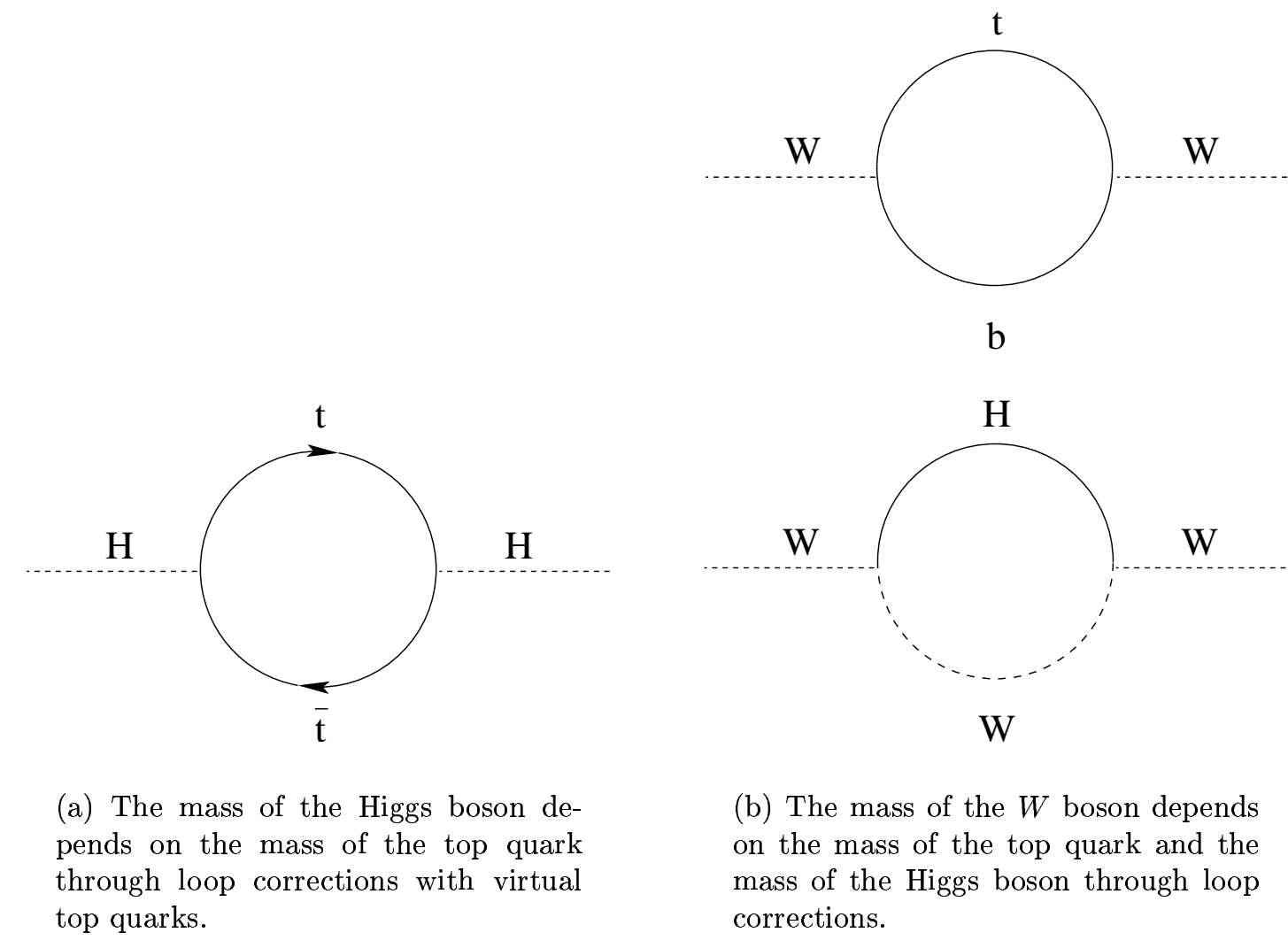

Figure 2.9: Loops corrections to the mass of the Higgs boson and the mass of the $W$ boson. 


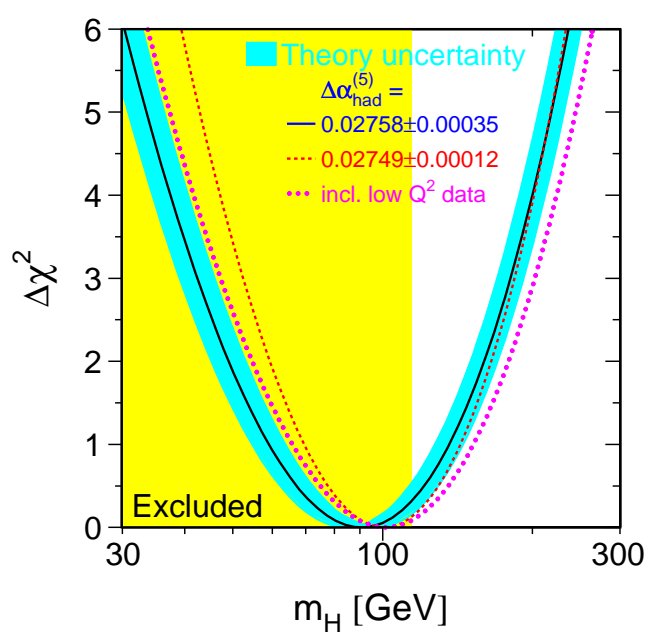

(a) Blueband plot, showing the indirect measurement of the Higgs boson mass from all electro-weak data together with the $95 \%$ confidence lower limit on the Higgs mass of $114.4 \mathrm{GeV} / c^{2}$ [9].

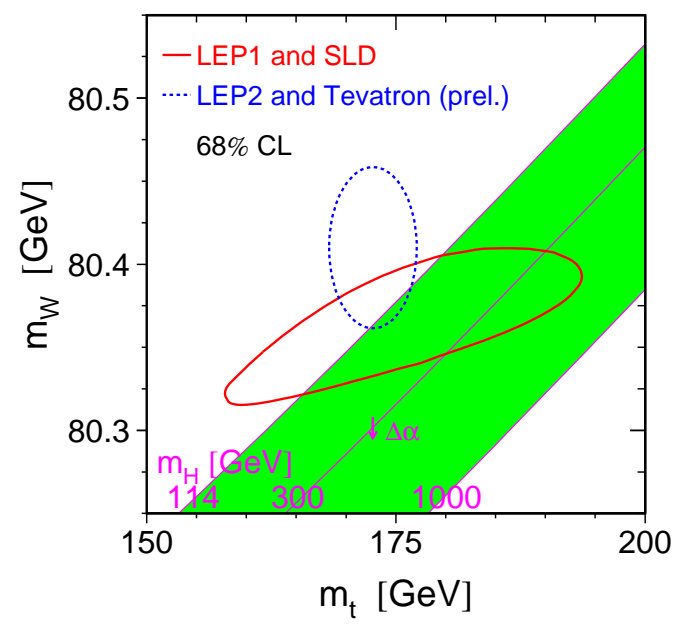

(b) Contour curves of $68 \%$ confidence level in the $\left(m_{t}, m_{W}\right)$-plane, for the indirect (LEP1, SLD data) and direct (LEP2, $p \bar{p}$ data) determination in a global fit to electro-weak precision data [9]. Also shown is the expected correlation between $m_{t}$ and $m_{W}$ in the SM for different Higgs boson masses.

Figure 2.10: Constraints on the mass of the Higgs boson from electro-weak precision fits. 



\section{Chapter 3}

\section{The $\mathrm{D} \emptyset$ Detector}

The $\mathrm{D} \varnothing$ detector [19], shown in Fig. 3.1, is a typical multi-purpose high energy physics detector. It is capable of identifying a variety of objects, including electrons, muons and hadronic jets. This chapter describes the subdetectors of the $\mathrm{D} \varnothing$ detector, the three levels of the trigger system and the data acquisition system.

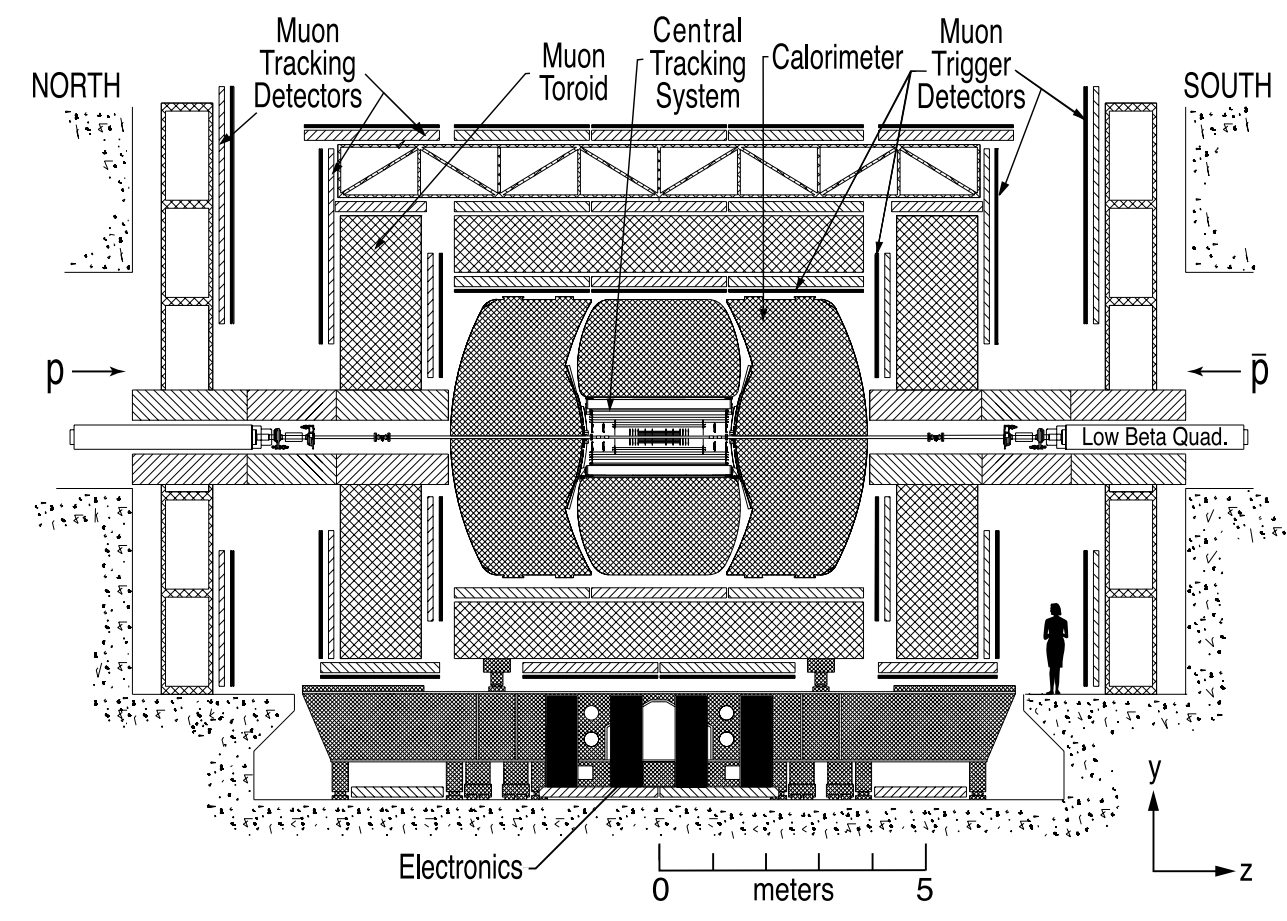

Figure 3.1: Cross section of the $D \varnothing$ detector in the vertical plane. The definition of the $\mathrm{D} \varnothing$ coordinate system is shown in the lower right corner.

The definition of the $\mathrm{D} \emptyset$ coordinate system is shown in Fig. 3.1. The positive $z$ axis points along the direction of the proton beam, the positive $x$-axis points radially 
outward from the center of the Tevatron and the positive $y$-axis is directed upwards. Other often used coordinates are the standard polar coordinates:

$$
\begin{aligned}
r & =\sqrt{x^{2}+y^{2}} \\
\theta & =\arccos \frac{z}{\sqrt{x^{2}+y^{2}+z^{2}}} \\
\phi & =\arctan \frac{x}{y}
\end{aligned}
$$

Since the angle $\theta$ is not invariant under Lorentz transformations along the $z$-axis, it is more common to use the rapidity $y$

$$
y=\frac{1}{2} \ln \left(\frac{E+p_{z}}{E-p_{z}}\right)
$$

or the pseudorapidity $\eta$

$$
\eta=-\ln \left(\tan \frac{\theta}{2}\right) .
$$

The pseudorapidity is equal to the rapidity when particle masses are neglected. Neglecting the mass is appropriate for highly relativistic particles. The term "forward" is used to describe objects or regions at large $|\eta|$. The separation between two directions, labeled 1 and 2, can be expressed as the distance $\Delta \mathcal{R}$ between them in the $(\eta, \phi)$-plane, defined as

$$
\Delta \mathcal{R}=\sqrt{\left(\eta_{1}-\eta_{2}\right)^{2}+\left(\phi_{1}-\phi_{2}\right)^{2}} .
$$

The separation $\Delta \mathcal{R}$ is a Lorentz invariant quantity.

\subsection{Charged Particle Identification}

To be able to identify charged particles and measure their charge and momenta, the $\mathrm{D} \varnothing$ detector is equipped with a tracking system inside a $2 \mathrm{~T}$ magnetic field provided by a solenoid magnet. The tracking detectors $[21,51]$ are located closest to the beam pipe. Their central location enables precise measurements of the primary interaction point and the impact parameter of charged particles. The magnetic field bends the trajectory of charged particles in the $(x, y)$-plane as they pass through the tracking detectors. The momenta of the particles are inferred from the radius of their trajectory:

$$
p_{T}[\mathrm{GeV} / c]=0.3 \cdot r[\mathrm{~m}] \cdot B[\mathrm{~T}] .
$$

To get an accurate estimate of the curvature it is desirable to have many measurements along the particle trajectory as well as a long lever arm. This is achieved by combining a high resolution silicon based device closest to the beam pipe, surrounded by a larger detector based on scintillating fibers. The D $\varnothing$ tracking system is shown in Fig. 3.2. 


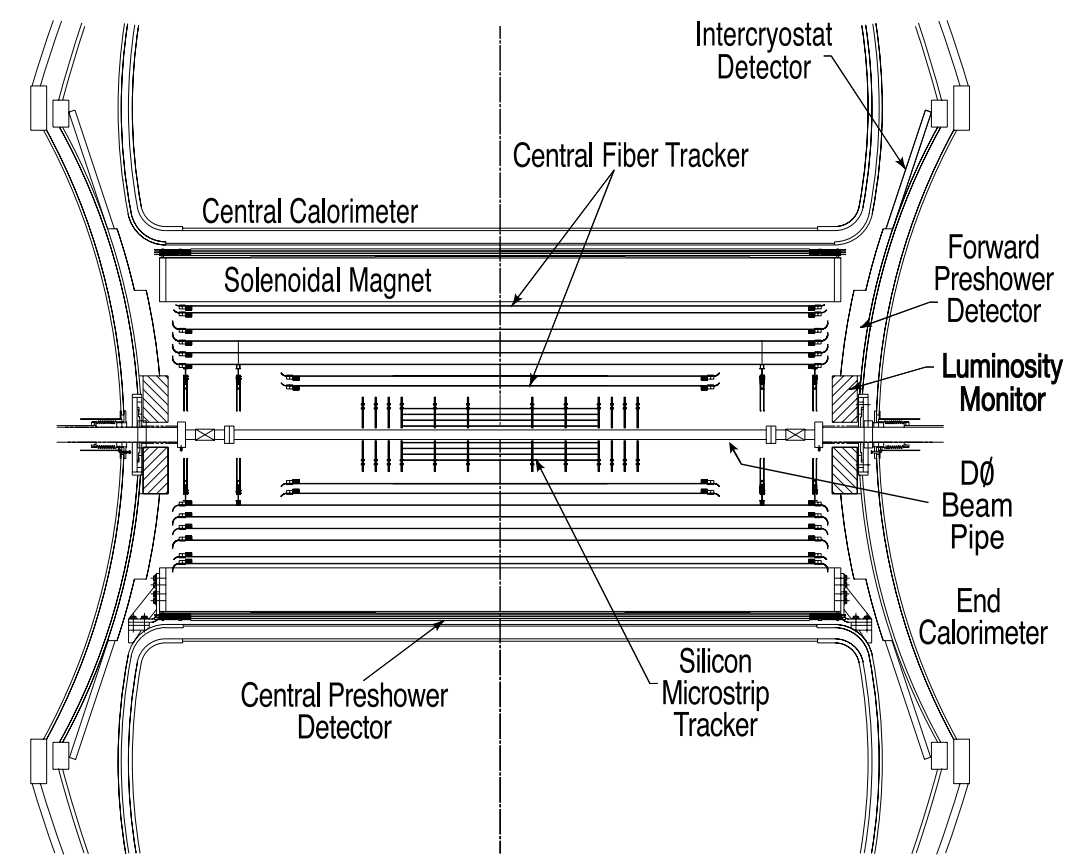

Figure 3.2: The D $\varnothing$ tracking system. Also shown are the locations of the solenoid, the preshower detectors, luminosity monitors and the calorimeters.

The Silicon Microstrip Tracker (SMT) is the high resolution part of the tracking system. It is especially important for vertex reconstruction and precise momentum measurements. The Central Fiber Tracker (CFT) is located outside of the SMT. The CFT provides several measurement points along the particle trajectory with higher efficiency but lower resolution than the SMT. Surrounding the CFT is the $2.8 \mathrm{~m}$ long superconducting solenoid with a radius of $60 \mathrm{~cm}$.

High precision tracking is necessary to reconstruct secondary decay vertices from $B$-hadrons. The $B$-hadrons have a lifetime of $\mathcal{O}(1)$ ps which translates into a decay length of $\mathcal{O}(1) \mathrm{mm}$. This is enough to separate the $B$-hadron decay from the primary interaction. This technique allows for the identification of jets coming from $b$ quarks, so called $b$-jets. The identification of $b$-jets is an important tool when looking for the decay products of top quarks.

\subsubsection{The Silicon Microstrip Tracker}

The design of the SMT [21] is driven by the physics requirements of top quark physics and $B$-physics. The high mass of top quarks implies that narrow jets with a large number of tracks are produced in the central part of the detector. High position resolution is therefore essential. It is also desirable to cover a large solid angle. Since the luminous region extends over $60 \mathrm{~cm}$ in $z$, the SMT has to cover a 
significant region in this direction.

To meet these criteria the SMT is built up of six barrels extending out to $|z|=40$ $\mathrm{cm}, 12$ smaller F-disks placed in between and just outside the barrels, and four larger H-disks located at $|z|=100 \mathrm{~cm}$ and $|z|=121 \mathrm{~cm}$, as shown in Fig. 3.3. Tracks with low $|\eta|$ are measured primarily in the barrels while forward tracks pass through the disks. The barrels and disks consist of silicon microstrip detectors with a hit resolution of approximately $10 \mu \mathrm{m}$. The impact parameter resolution for central tracks is approximately $15 \mu \mathrm{m}$. With the H-disks the SMT covers a region up to $|\eta|=3$.

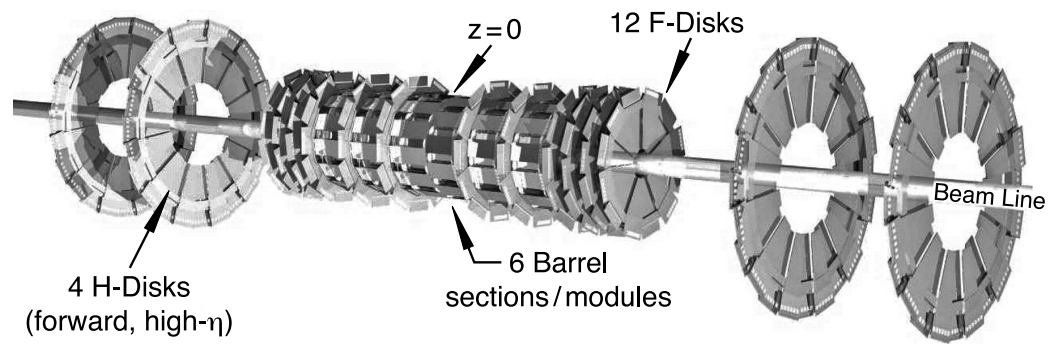

Figure 3.3: The DØ Silicon Microstrip Tracker. There are six barrels, 12 smaller F-disks located in between and just outside the barrels and 4 larger H-disks.

The smallest detector element consists of a silicon sensor and its readout chips. The detector elements are called ladders in the barrels and wedges in the disks. Figure 3.4 shows the layout of a ladder.

Each barrel in the SMT consists of eight layers, where two layers are needed to provide full coverage in $\phi$, as shown in Fig. 3.5(a). The eight layers are evenly arranged between radii of $2.7 \mathrm{~cm}$ and $9.4 \mathrm{~cm}$ and are numbered from 1 to 8 with increasing radius. Layers 1-4 are made up of 6 ladders per layer whereas layers 5-8 have 12 ladders per layer. The F-disks contain 12 wedges, and the H-disks are made up of 24 wedges. A drawing of an F-disk is shown in Fig. 3.5(b). The inner radius of the F-disks is $2.6 \mathrm{~cm}$ and the wedges extend $7.5 \mathrm{~cm}$ in the radial direction. The $\mathrm{H}$-disks have an inner radius of $9.5 \mathrm{~cm}$ and the wedges are $14.6 \mathrm{~cm}$ long.

There are three types of ladders and two types of wedges, which are all read out by SVXIIe chips [52]. Each chip is connected to 128 silicon strips, with widths varying from 50-150 $\mu \mathrm{m}$. The different types of ladders are called 3-chip, 6-chip and 9-chip ladders, named after their number of readout chips. The 3-chip ladders are single-sided and are located in the two outermost barrels in layers 1, 2, 5 and 6 . The 6-chip ladders are double-sided sensors with a stereo angle of $90^{\circ}$. They are located in the four inner barrels in layers 1,2,5, and 6. Layers 3,4,7 and 8 of all barrels are populated by the 9-chip ladders. These are double-sided detectors with a stereo angle of $2^{\circ}$. The wedges in the F-disks are double sided with a stereo angle of $15^{\circ}$ per side (the strips are at an angle of $30^{\circ}$ with each other) while the wedges 

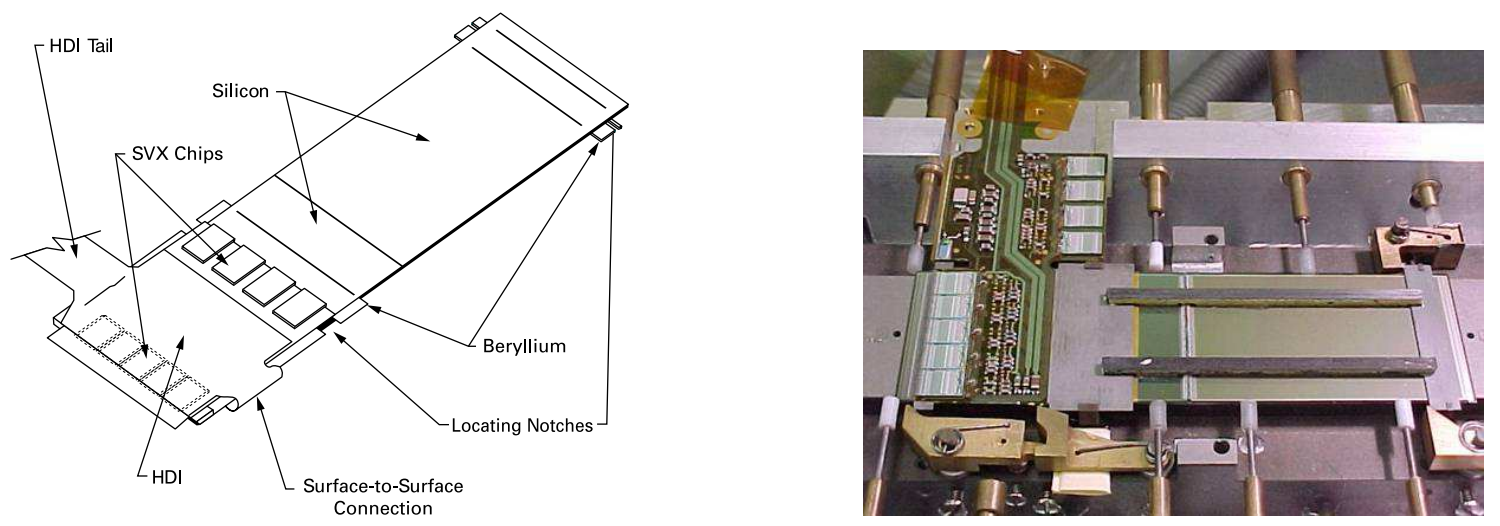

Figure 3.4: Drawing of a silicon ladder (left) and a photo of a double sided ladder during assembly of the silicon onto the unfolded High Density Interconnect, HDI (right).

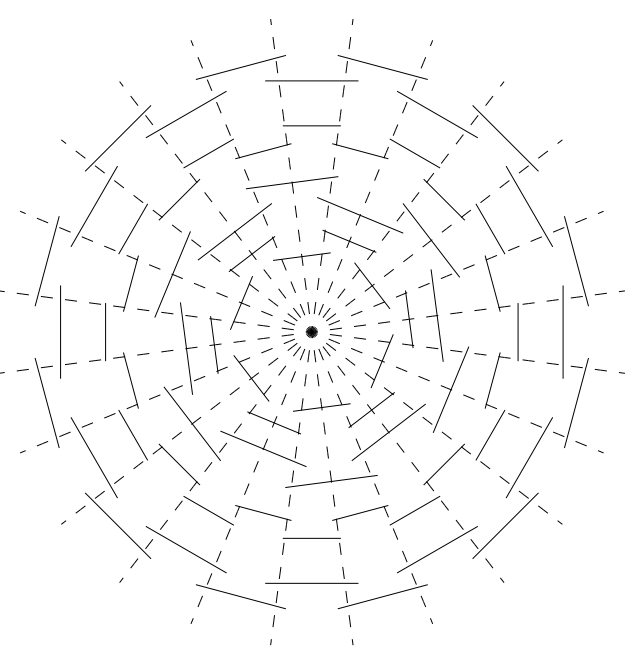

(a) Drawing of the ladder structure in an SMT barrel. A barrel consists of eight layers. Two layers are needed to provide a complete coverage in $\phi$.

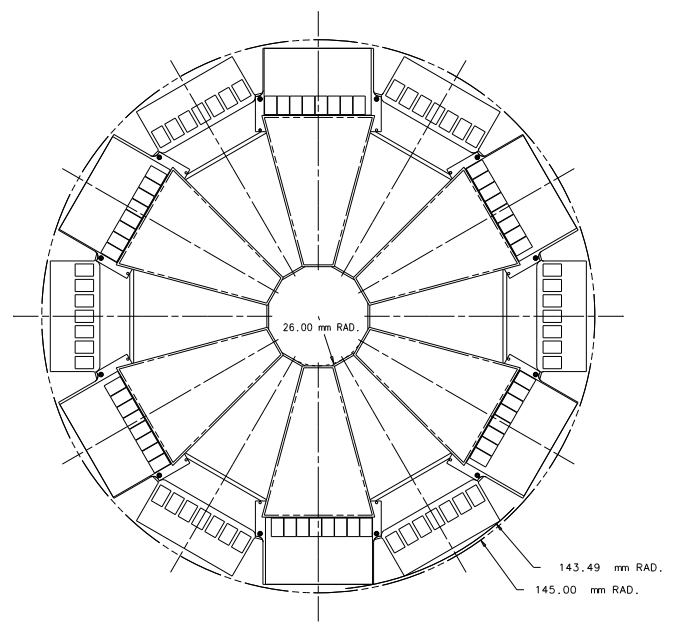

(b) A drawing of the F-disk layout in the $(r, \phi)$-plane.

Figure 3.5: Layouts of the SMT barrels and disks, viewed in the $(r, \phi)$-plane. 
in the H-disks consists of two single-sided detectors with stereo angles of $15^{\circ}$ glued together.

The SVXIIe chip was designed specifically for Run II and is used to read out both the SMT and the CFT. The chip collects the charge on every strip and digitizes the signal. The collected signal in each channel is then compared to a hit threshold which is common for all 128 channels. If the signal exceeds the threshold the strip is signaled as a hit strip. Since the SMT has approximately 793,000 readout channels, the event size grows too large if all channels are read out in every event. Therefore only the hit strips and their neighboring strips are read out.

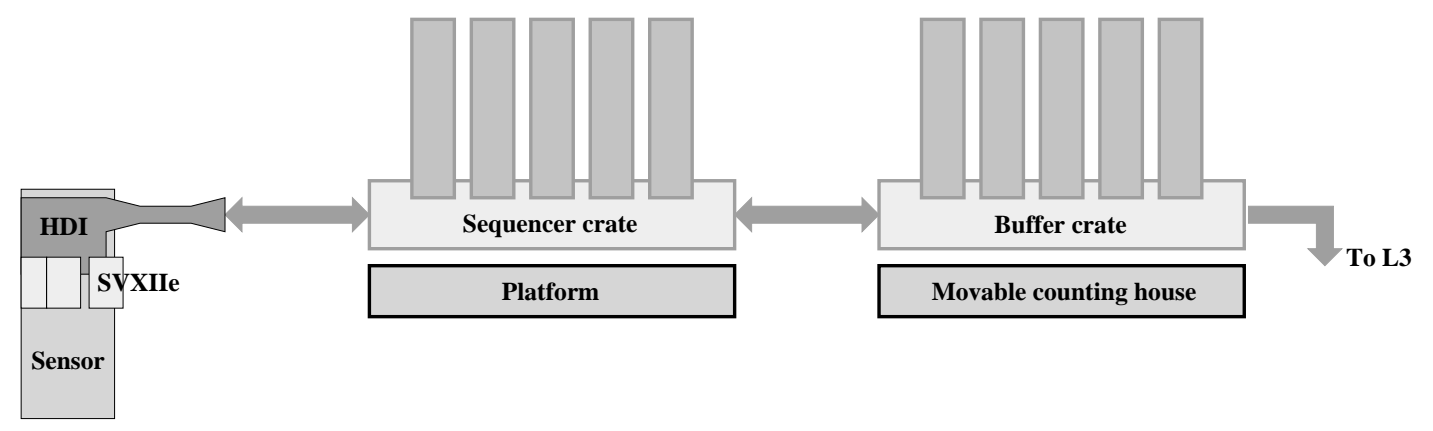

Figure 3.6: Cartoon of the major components in the SMT readout chain. The silicon sensors are read out by SVXIIe chips. The chips are controlled by sequencers which are located under the $\mathrm{D} \varnothing$ detector in an area called the "Platform". The sequencers relay the information to buffer crates once a Level 1 trigger accept occurs. If the event passes the Level 2 trigger the information is passed on to the Level 3 trigger system.

The sequencer, shown in Fig. 3.6, controls the SVXIIe chips and is also responsible for relaying the readout to the buffer crates for storage once a Level 1 trigger accept occurs. The buffer crates stores the readout until a Level 2 trigger accept has been issued and then passes the information on to the Level 3 trigger system. If the event is rejected at Level 2 the information is discarded. In the Level 3 trigger system the information from the SMT is used to reconstruct the charged particle tracks in the event.

\subsubsection{The Central Fiber Tracker}

The CFT [19] consists of eight cylindrical layers of fibers and occupies the radial space from 20 to $52 \mathrm{~cm}$ from the center of the beampipe. To accommodate the forward H-disks in the SMT, the two innermost layers are $1.66 \mathrm{~m}$ long whereas the six outer layers are $2.52 \mathrm{~m}$ long, as shown in Fig. 3.2. This corresponds to an $\eta$ coverage of $|\eta|<1.7$. 
Each layer of fibers consists of two sub-layers, one with fibers along the beam direction $(z)$ called an axial layer and one with the fibers at a stereo angle in $\phi$ of $+3^{\circ}(u)$ or $-3^{\circ}(v)$. The $u$ and $v$ types of layers are called stereo layers. Figure 3.7 illustrates the difference between $z, u$ and $v$ layers. From the smallest cylinder

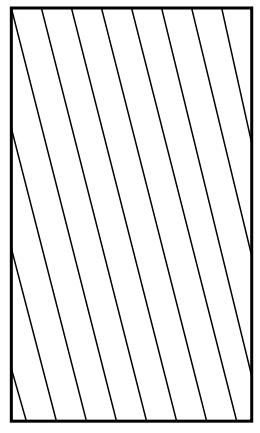

u

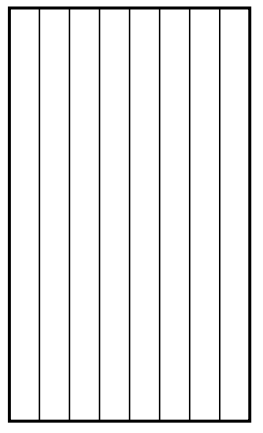

Z

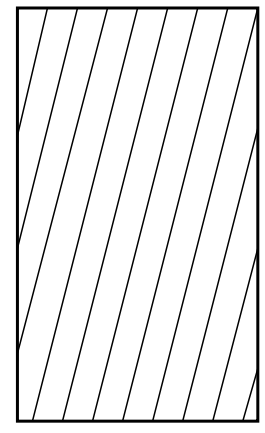

V

Figure 3.7: Layers with fibers directed along the $z$-axis are called axial layers $(z)$. Layers where the fibers are inclined with respect to the $z$-axis are called stereo layers $(u$ and $v)$.

outwards, the fiber orientation is $z u-z v-z u-z v-z u-z v-z u-z v$. Each sublayer (axial or stereo) in turn consists of two layers of $835 \mu \mathrm{m}$ diameter scintillating fibers. One layer is offset by half the fiber spacing with respect to its partner, removing all gaps. A drawing of the eight axial layers is shown in Fig. 3.8. The small fiber diameter gives the CFT a hit resolution of approximately $100 \mu \mathrm{m}$ for each of the axial or stereo layers.

The light signal from the fibers are transported through clear fiber waveguides and converted to electrical signals in the Visible Light Photon Counters (VLPCs). The VLPCs are avalanche photodetectors made of impurity-band silicon and capable of detecting single photons. They are operated at $9 \mathrm{~K}$ and provide fast response and high gain. The CFT has a total of 76,800 channels of VLPC readout.

The signal from the VLPCs are digitized by the SVXIIe chip mounted on Analog Front-End (AFE) boards and controlled by sequencers. On the AFE boards the analog signal is sampled before digitization and used in the Level 1 central track trigger. Only the axial layers are used for triggering.

\subsection{Electromagnetic and Hadronic Showers}

Outside the solenoid there are a number of calorimeter detectors aimed at measuring the energy of electrons, photons and jets of hadrons. When these particles traverse matter they lose energy. The energy loss depends on the type of particle and on the atomic properties of the material. 


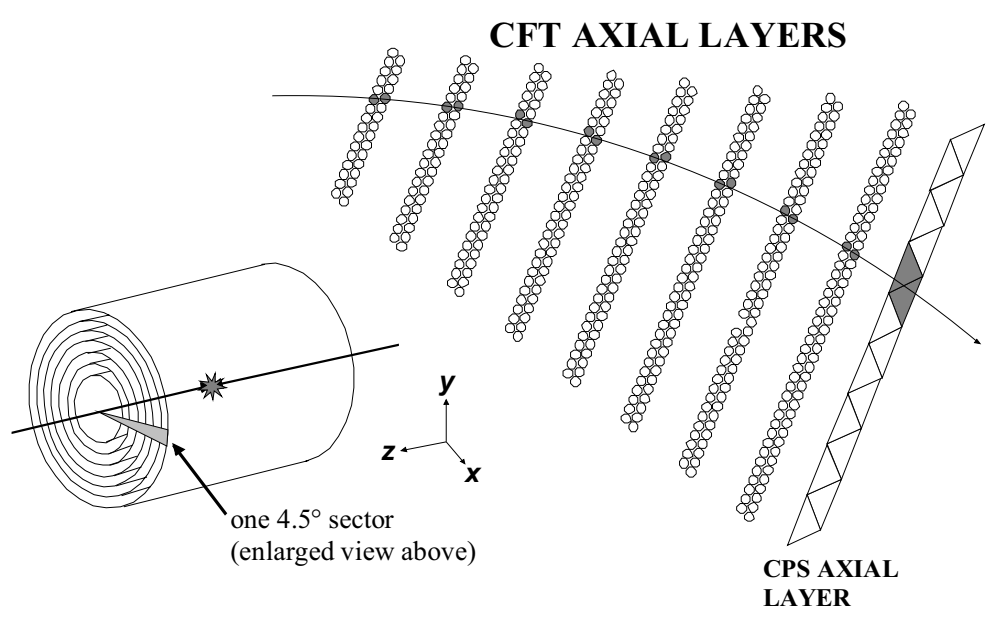

Figure 3.8: A $4.5^{\circ}$ wedge of the CFT, showing the eight axial layers. Each axial layer consists of two layers of fibers, one layer offset by half the fiber spacing with respect to its partner. Outside each axial layer there is a stereo layer which is not shown in this picture.

Electrons passing through matter lose energy primarily through ionization and through bremsstrahlung. Above the critical energy $E_{c}$ bremsstrahlung is the dominant process. The critical energy can be approximated by [22]

$$
E_{c}=\frac{800}{Z+1.2} \mathrm{MeV},
$$

where $Z$ is the atomic number. The emitted photons produce $e^{+} e^{-}$pairs, which in turn emit photons. The resulting shower of electrons and photons grows until the energy of the electrons falls below the critical energy, where they primarily interact through ionization. The mean distance over which an electron loses all but $1 / e$ of its energy is called the radiation length $X_{0}[22]$,

$$
X_{0}=\frac{716.4 A}{Z(Z+1) \ln (287 / \sqrt{Z})} \mathrm{g} \mathrm{cm}^{-2},
$$

where $A$ is the atomic mass of the medium in $\mathrm{g} \mathrm{mol}^{-1}$.

Photons interacting with matter will produce $e^{+} e^{-}$pairs, creating an electromagnetic shower in the calorimeter. Since the photons are not electrically charged they will not leave a track in the tracking detectors. This is the main signature which distinguishes photons from electrons.

Hadronic particles passing through matter will interact inelastically with nuclei to produce primarily pions and nucleons. At high energies the produced particles will in turn interact with nearby nuclei to produce a shower of hadronic particles. 
The characteristic length scale is the nuclear interaction length $\lambda_{I}[22]$ :

$$
\lambda_{I} \approx 35 \cdot A^{1 / 3} \mathrm{~g} \mathrm{~cm}^{-2}
$$

where $A$ is the atomic mass of the medium in $\mathrm{g} \mathrm{mol}^{-1}$. A significant fraction of the energy of the initial hadron escapes the hadronic cascade. The main sources of energy loss are through unbinding of nuclei by spallation, non-ionizing collisions and neutrino production.

\subsubsection{The Preshower Detectors}

The preshower detectors, shown in Fig. 3.2, provides an early energy sampling of showers as well as a precise position measurement. They are scintillator based devices and function as hybrids between tracking detectors and calorimeters. Their main purpose is to aid in identification of electrons and photons in the trigger decision and in the offline event reconstruction. The preshower detector consists of a central and a forward part, located in between the solenoid and the calorimeter.

The Central Preshower detector (CPS) is mounted on the solenoid and covers the region of $|\eta|<1.3$ with an inner radius of $72 \mathrm{~cm}$ and an outer radius of 74 $\mathrm{cm}$. The solenoid, which is $0.9 X_{0}$ thick, is followed by a $\mathrm{Pb}$ radiator approximately one radiation length thick. Outside the $\mathrm{Pb}$ radiator are three layers of triangular scintillator strips. The solenoid and $\mathrm{Pb}$ radiator converts electrons and photons into showers which are sampled in the scintillators. This provides a good discrimination against charged pions which pass through the radiator without showering. The scintillator strips in the three layers are arranged in a $z-u-v$ geometry. The $u$ layer has a stereo angle of $+23.8^{\circ}$ and the $v$ layer a stereo angle of $-24.0^{\circ}$. Embedded in the center of each triangular strip is a fiber that collects the light signal and transports it to the readout. Figure 3.9 shows a cross section of a scintillator layer.

The Forward Preshower detector (FPS) sits on the end calorimeter cryostats and covers the region $1.5<|\eta|<2.5$, as shown in Fig. 3.2. Closest to the interaction region in $z$ are two layers of scintillator strips in a $u-v$ configuration. The stereo angles of the two layers are $\pm 22.5^{\circ}$. Charged particles register a single three dimensional hit in these layers. Following the scintillator layers is a $2 X_{0}$ thick lead-stainless-steal absorber. Outside the absorber are two additional scintillator layers in the same $u-v$ configuration as the first two layers of scintillators. Electrons and photons will shower in the absorber leading to a cluster of energy in the outer scintillator layers.

The light collected in the scintillator strips is carried through the readout fibers and converted into electrical signals by VLPCs. The VLPCs are interfaced to AFE boards in the same configuration as in the CFT. The downstream readout is also common to both the SMT and the CFT. The fast readout allows the preshower detectors to be part of the trigger system at all levels. Only the axial preshower layers are used in the Level 1 trigger. The geometry of the CPS matches that of the outer layer of the CFT for triggering purposes. The stereo layers are used in Level 2 

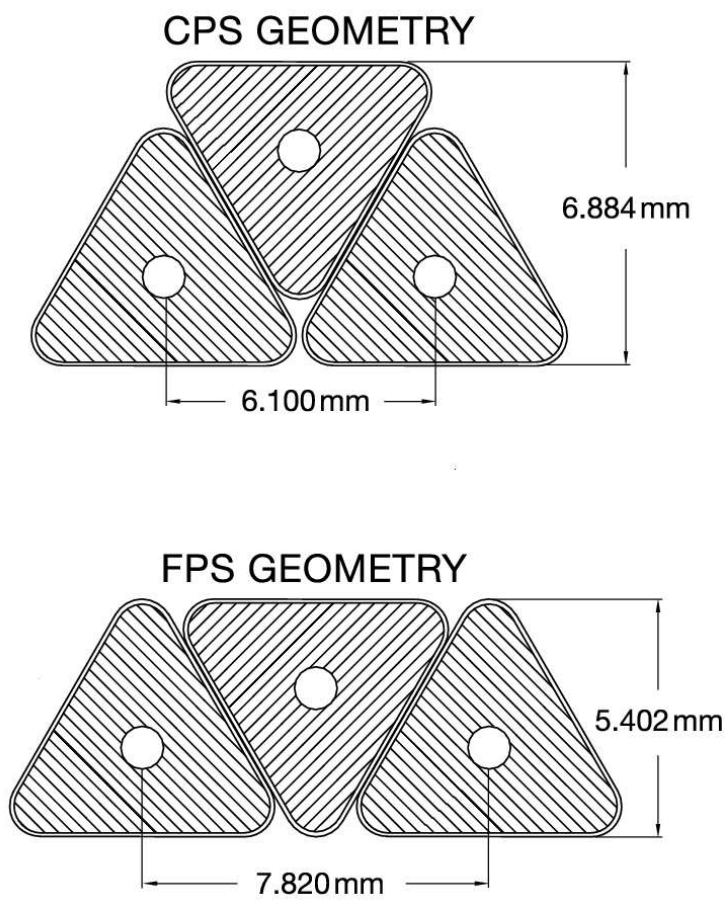

Figure 3.9: Cross section of a layer in the CPS and the FPS. The circles inside the triangular scintillators show the location of the fibers used to read out the light signals.

and Level 3 of the trigger.

\subsubsection{The Calorimeter}

The calorimeter absorbs electrons, photons and hadrons and measures their energy. The calorimeter is crucial for the reconstruction of hadronic jets from the fragmentation of quarks and gluons. The direction of neutrinos in the event can be inferred from the measured momentum imbalance in the transverse plane. The calorimeter is used in all levels of the trigger system to select events with electrons, photons and high $p_{T}$ jets.

The $\mathrm{D} \varnothing$ calorimeter is a hermetic and radiation-hard liquid argon sampling calorimeter in three parts. The central calorimeter and the two end detectors are shown in Fig. 3.10(a). From the center of the D $\varnothing$ detector and outwards they contain an electromagnetic section (EM), a fine hadronic section (FH) and a coarse hadronic section $(\mathrm{CH})$. In order to achieve the same energy response for electromagnetic and hadronic particles, $e / h \approx 1$ (compensating calorimeter), different absorber plates are used at different locations. The active medium is liquid argon in all the calorimeters. Each calorimeter is located within a cryostat which keeps the operat- 
ing temperature at $90 \mathrm{~K}$. In between the central and forward calorimeter cryostats there is a region with reduced coverage.

The central calorimeter (CC) provides coverage out to $|\eta| \leq 1.0$. Nearly depleted uranium is used as the absorber in the EM sections. The thickness is different for all four EM layers. From the innermost layer and outwards they are approximately 1.4, 2.0, 6.8 and $9.8 X_{0}$ thick. One radiation length in uranium corresponds to approximately $3 \mathrm{~mm}$. Uranium is used as the absorber material due to its high density and compensating response. In the $\mathrm{FH}$ section a uranium-niobium (2\%) alloy is used as absorber material. The three layers correspond to approximately 1.3, 1.0 and $0.76 \lambda_{I}$. The nuclear interaction length $\lambda_{I}$ is much longer than the radiation length. In uranium

$$
1 \lambda_{I}^{U r} \approx 10.5 \mathrm{~cm} \approx 30 X_{0}^{U r} .
$$

In the single coarse hadronic layer copper is used as the absorber material with a total thickness of $3.2 \lambda_{I}$.

The forward calorimeters (EC) extend the acceptance out to $|\eta| \approx 4.0$, as shown in Fig. 3.10(b). In the four EM layers depleted uranium is used as absorber material with a thickness of 1.6, 2.6, 7.9 and $9.3 X_{0}$. The EC hadronic calorimeter consists of three concentric parts as shown in Fig. 3.10(a). A uranium-niobium alloy is used as absorber material in the $\mathrm{FH}$ section and stainless steel is used in the $\mathrm{CH}$ section. The two EC inner hadronic modules are cylindrical with an inner and outer radii of 3.92 and $86.4 \mathrm{~cm}$. Each of the four FH layers is $1.1 \lambda_{I}$ thick. The $\mathrm{CH}$ section consists of a single layer which is $4.1 \lambda_{I}$ thick. The EC middle hadronic modules have four FH layers, each $0.9 \lambda_{I}$ thick, and a single $\mathrm{CH}$ section with a thickness of $4.1 \lambda_{I}$. The outer hadronic modules are made from stainless steel with a maximum thickness of $6.0 \lambda_{I}$.

The calorimeter readout cells form towers, as shown in Fig. 3.10(b). The transverse size of the readout cells are comparable to the transverse sizes of showers, 1-2 $\mathrm{cm}$ for electromagnetic showers and about $10 \mathrm{~cm}$ for hadronic showers. Towers in both EM and hadronic sections cover an area of $\Delta \eta=0.1$ and $\Delta \phi=2 \pi / 64 \approx 0.1$. The third layer of the EM modules, located at the maximum of the electromagnetic shower, is segmented twice as finely in both $\eta$ and $\phi$ to allow for a more precise reconstruction of the location of the shower center. There are a total of 47,032 readout cells in the calorimeter.

Between the central and forward calorimeter cryostats there is a region with reduced coverage. A significant amount of unsampled material from the SMT and CFT cables which are routed through this region degrades the energy resolution. Additional sampling is achieved with layers of scintillator detectors attached to the exterior surfaces of the end cryostats. They cover the region $1.1<|\eta|<1.4$ divided into tiles each covering $\Delta \eta \times \Delta \phi \approx 0.1 \times 0.1$.

The energy resolution of the electromagnetic and hadronic modules was studied in Run I using pions and electrons from a test beam [53] with energies between 10 


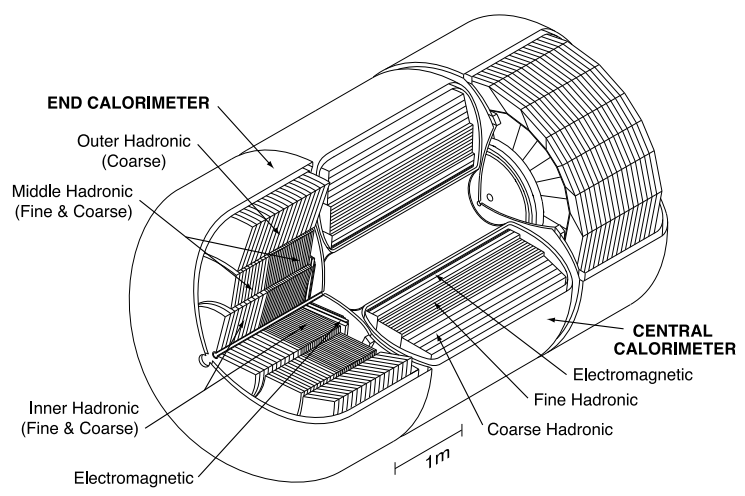

(a) Isometric view of the central calorimeter and the two end calorimeters.

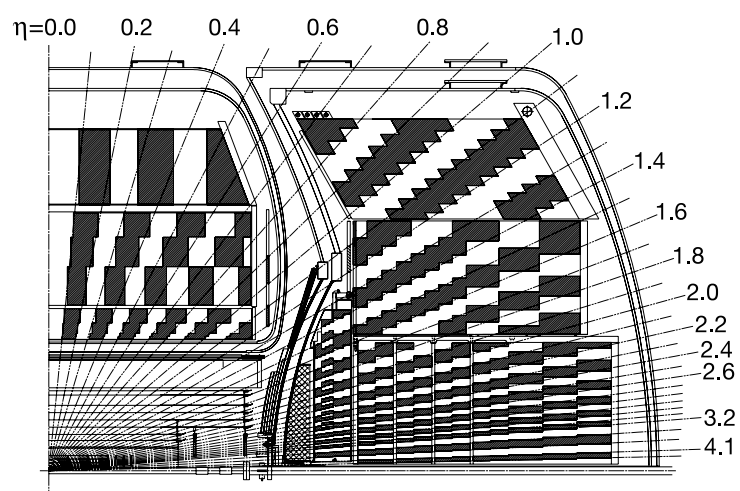

(b) Drawing of one quadrant of the $\mathrm{D} \emptyset$ calorimeter. The shading pattern indicates groups of cells ganged together into readout towers. The rays indicate pseudorapidity intervals from the center of the detector.

Figure 3.10: Drawings of the D $\varnothing$ calorimeter.

and $150 \mathrm{GeV}$. The energy resolution can be parametrized as:

$$
\frac{\sigma(E)}{E}=\sqrt{C^{2}+\left(\frac{S}{\sqrt{E}}\right)^{2}+\left(\frac{N}{E}\right)^{2}} .
$$

The dominant term $S$ in the energy resolution is due to sampling fluctuations. The noise term $N$ models the uranium and readout electronics noise. The constant term $C$ originates from calibration errors and other systematic effects. The numerical values for $S, N$ and $C$ found in the test beam study are given in Tab. 3.1.

\begin{tabular}{cccc}
\hline Particle & $C$ & $S$ & $N$ \\
\hline$e$ & $0.0115_{-0.0036}^{+0.0027}$ & $(0.135 \pm 0.005) \sqrt{\mathrm{GeV}}$ & $0.43 \mathrm{GeV}$ \\
$\pi$ & $0.032 \pm 0.004$ & $(0.45 \pm 0.04) \sqrt{\mathrm{GeV}}$ & $0.975 \mathrm{GeV}$ \\
\hline
\end{tabular}

Table 3.1: Calorimeter energy resolution parameters measured in the Run I test beam data [53]. Uncertainties on $N$ were not determined.

The energy resolution of the hadronic calorimeter in Run II can be measured in random collisions without trigger bias (so called "zero-bias" events). The central tracking system can be used to identify single tracks from isolated charged particles which are predominately charged pions. The tracking system provides a momentum 
measurement which can be used to determine the expected energy deposition in the calorimeter. Preliminary studies [54] shows a degraded resolution compared to Run I, mainly due to the following reasons:

- The readout electronics of the calorimeter was upgraded to account for the higher interaction rate of the Tevatron in Run II. The new electronics is found to have a worse noise performance, affecting mainly the $N$ term in Eq. 3.8.

- The higher interaction rate in Run II gives a much shorter integration time for the readout electronics. Only about $70 \%$ of the signal charge can be sampled which leads to a larger stochastic terms. In particular the contribution from slow nuclear products from the hadronic shower is lost. This degrades the values for the $S$ and $N$ terms in Eq. 3.8.

- The new central tracking system, the solenoid magnet and the preshower detector installed for Run II introduces a total of 2-4 $X_{0}$ of material in front of the calorimeter. As a consequence the $S$ term in Eq. 3.8 degrades.

\subsection{The Muon System}

The muon system is the outermost detector system. Its main purpose is to measure the trajectory of muons, which are the only charged particles traversing the calorimeter system.

The muon system [55], shown in Fig. 3.11, is divided into a central part called the wide angle muon spectrometer (WAMUS) covering the region out to $|\eta|=1$ and the forward angle muon spectrometer (FAMUS) providing coverage in the range $1<|\eta|<2$. Both the central and the forward detectors have three layers of wire chambers which are used for precise coordinate measurements and triggering. For trigger and timing purposes there are two or three layers of scintillator detectors interspaced between the layers of wire chambers. From the innermost layer and outwards, the three layers are called A, B and C. Between layers A and B is a toroid magnet which provides a $1.8 \mathrm{~T}$ magnetic field bending the muon trajectories in the $(r, z)$-plane. The magnetic field enables a standalone measurement of the muon momenta. This improves the momentum resolution for high momentum muons and also allows for a cleaner matching with the tracks in the central tracking system.

The B- and C-layers in the central muon system consist of three decks of proportional drift tubes (PDTs). The A-layer has four decks, except the bottom A-layer which has only three decks. The drift chambers are large, typically $2.8 \times 5.6 \mathrm{~m}^{2}$ and have a hit resolution between 10-50 cm depending on how close the hit is to the readout electronics. The scintillators covering the A-layer are called $A \phi$ scintillator counters. They provide a fast signal for triggering on muons and aid in the identification of muons. There are also scintillators installed on the top, side and bottom of the C-layer PDTs called cosmic cap and bottom counters. They provide a fast 


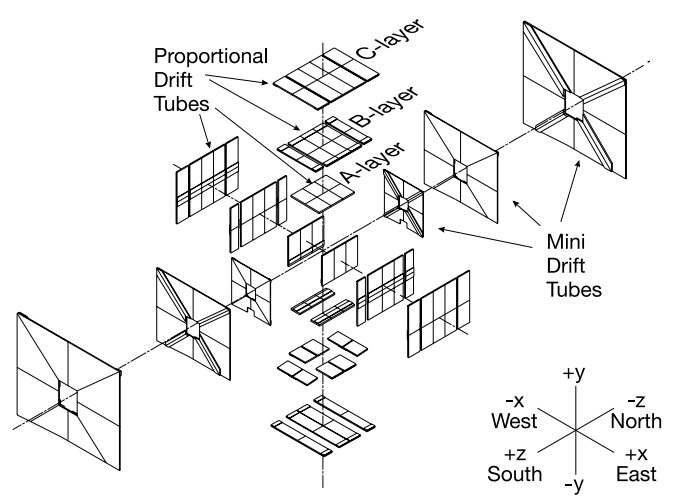

(a) Exploded view of the muon wire chambers.

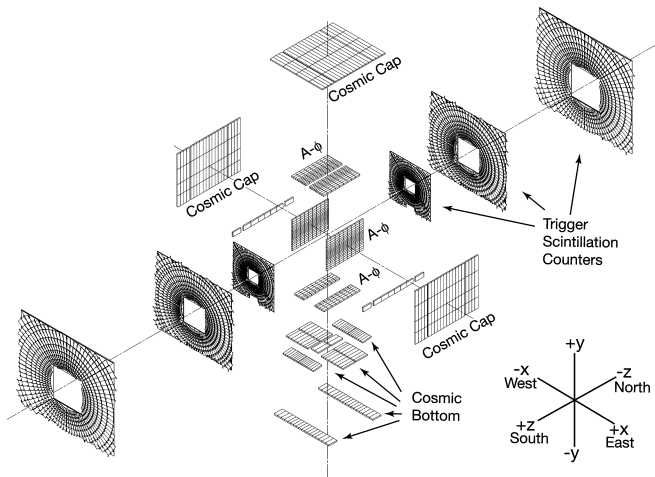

(b) Exploded view of the muon scintillator detectors.

Figure 3.11: The D $\varnothing$ muon system, which consists of wire chambers and scintillator detectors.

timing signal to associate a muon in a PDT with the appropriate bunch crossing and discriminate against the cosmic ray background.

The forward muon system consists of three layers of mini drift tubes (MDTs) and three layers of scintillators. The innermost layer, called layer A, is located inside the toroid whereas the B- and C-layers are located outside the toroid. The A-layer has four decks of MDTs and the B- and C-layers have three decks of MDTs. The scintillators are mounted on the inside of the A-layer MDTs and on the outside of the B- and C-layer drift tubes. The main function of the scintillators is to provide timing information and to assist in the trigger decisions.

\subsection{Trigger and Data Acquisition}

The vast majority of the collisions at the $\mathrm{D} \emptyset$ interaction region are so called minimum bias interactions of limited physics interest. The cross section for producing heavy particles such as top quarks, $W$ bosons or $Z$ bosons is much smaller than the total inelastic cross section for $p \bar{p}$ collisions as was shown in Fig. 2.3. To accumulate a large sample of interesting events without having to store and reconstruct a staggering number of uninteresting collisions, $D \varnothing$ uses an event trigger to decide whether to store an event. To handle the large event rate, the trigger system is divided into three distinct levels where each succeeding level examines fewer events but in greater detail and with more complexity.

The time between bunch crossings at the $\mathrm{D} \varnothing$ collision point is $396 \mathrm{~ns}$, corresponding to an event rate of $2.5 \mathrm{MHz}$. The offline processing capabilities allow for a final rate of $50 \mathrm{~Hz}$ for storage and reconstruction. The first stage of the trigger 
system (Level 1 or L1) stores the event in a pipeline which allows for a trigger decision within $4.2 \mu \mathrm{s}$. It reduces the initial rate to approximately $2 \mathrm{kHz}$ which is the input rate for the next trigger stage (Level 2 or L2). L2 makes a trigger decision within $100 \mu$ s based on more refined event information. This reduces the event rate to $1 \mathrm{kHz}$. The final trigger stage (Level 3 or L3) uses a collection of approximately 100 fast processors to perform a fast reconstruction of the whole event. It makes a trigger decision within $50 \mathrm{~ms}$ and reduces the rate to $50 \mathrm{~Hz}$. The events passing the L3 trigger are stored on tape for offline reconstruction. Figure 3.12 summarizes the components of the L1 and L2 trigger and the input rate at each level of the trigger system. The components are described in greater detail below.

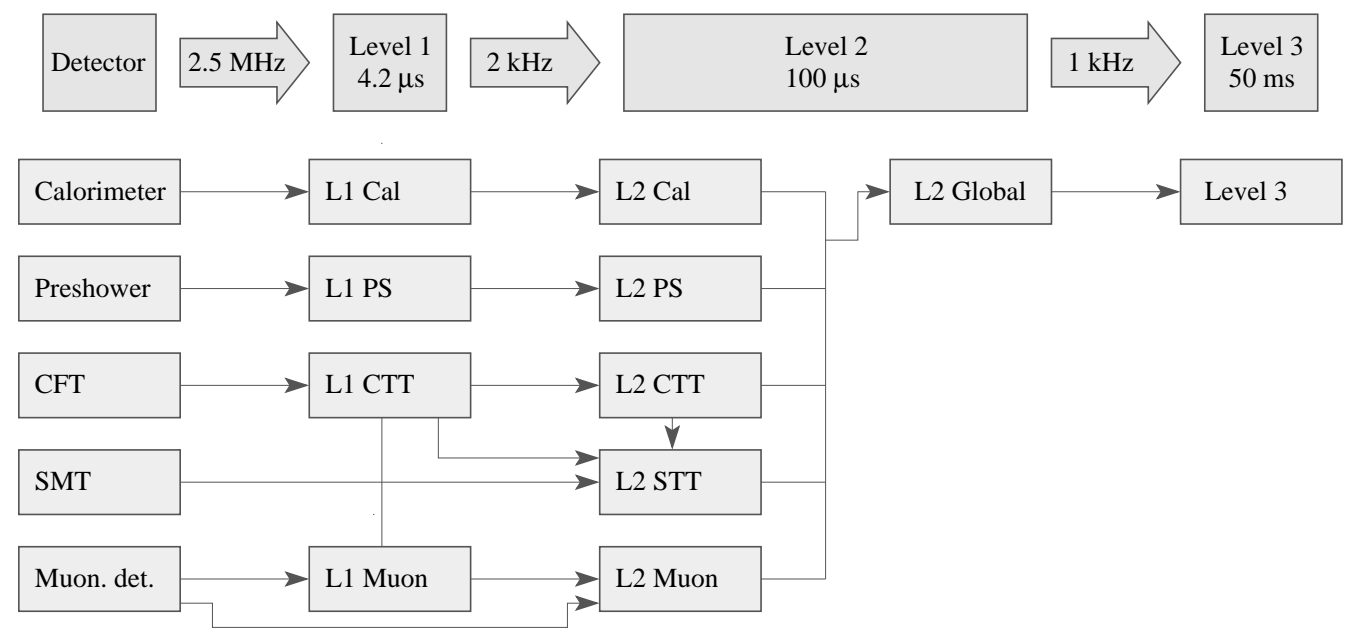

Figure 3.12: A summary of the components of the L1 and L2 trigger and the input rate at each level of the trigger system.

\subsubsection{The Level 1 Trigger}

The Level 1 trigger decisions are taken by dedicated electronics associated with the detector subsystems.

The calorimeter trigger decisions are taken based on the energy deposited in electromagnetic (EM) and hadronic (H) trigger towers of area $\Delta \eta \times \Delta \phi=0.2 \times 0.2$. The energy deposited in the tower is compared to a programmable trigger threshold. Separate thresholds can be used for the EM energy and the total EM+H energy. When measuring the hadronic energy the coarse hadronic section is not included due to its high noise level. The EM trigger towers are used to trigger on electrons and photons and the $\mathrm{EM}+\mathrm{H}$ trigger towers are used identify hadronic jets.

The Level 1 Central Track Trigger (CTT) uses information from the CFT and CPS axial layers. For every $4.5^{\circ}$ section in the transverse plane it compares the hit pattern with a set of 20,000 predefined track templates. An example of a hit pattern 
and a track template is shown in Fig. 3.8. Using predefined templates enables a fast response and the trigger is efficient for tracks with $p_{T}>1.5 \mathrm{GeV} / c$. The FPS axial layers are used to trigger on forward tracks.

The Level 1 muon trigger combine information from the muon wire chambers, the muon scintillator counters and the CTT. Three types of signatures are considered. A central track from the CTT can be matched to scintillator hits in one or more layers. A muon can also be identified from a track in the muon wire chambers which is confirmed with scintillator hits, or by requiring scintillator hits only.

\subsubsection{The Level 2 Trigger}

The Level 2 trigger decision is computed in two stages. First the information from the detector subsystems is refined. This is called the preprocessor stage. This information is then fed into a global processor, as shown Fig. 3.12, which makes the trigger decision. At the global stage data can be combined from the different subsystems into more accurate physics objects and event-wide correlations can be taken into account.

Jets are constructed from the clustering of $5 \times 5$ calorimeter trigger towers, centered on seed towers with $p_{T}>2.0 \mathrm{GeV} / c$. Electron and photon candidates are similarly constructed from $3 \times 3$ EM trigger towers centered on a seed tower with $p_{T}>1.0 \mathrm{GeV} / c$. Spatial matching with showers in the CPS or the FPS can be used to further enhance the electron identification capability. The Level 2 calorimeter trigger also computes the scalar and vector sum of the energy measured in all trigger towers. The scalar sum is used to trigger on events with high total transverse momentum, $H_{T}$. The vector sum can be used to estimate the missing transverse energy.

The L2 CTT takes the tracks from L1 as input, adds the information from the stereo layers, and refines the track measurements. The isolation of each track can be evaluated using several criteria. This enhances the capability to trigger on tau leptons.

The muon system preprocessors use calibration and more precise timing information to improve the quality of the muon candidates. The improved quality allows for better measurements of $p_{T}, \eta$ and $\phi$ of the muons.

\subsubsection{The Level 3 Trigger}

The Level 3 trigger is a fully programmable software trigger which performs a fast reconstruction of the event. The algorithms used to identify physics objects are comparable in complexity to those used in the offline reconstruction. L3 uses input from all detector subsystems. The hard scatter vertex is reconstructed with high accuracy and displaced vertices from the decays of long lived particles can be identified. 
The most important physics objects at the Level 3 trigger stage are:

- Jets: Using the precision calorimeter readout together with the knowledge of the hard scatter vertex greatly improves the energy resolution for jets compared with L1 and L2. The energy resolution is also improved from suppression of calorimeter cells which are known to have a high noise level.

- Electrons: Information from the calorimeter, preshower detectors and central tracking system is combined to identify electrons with high precision and purity.

- Muons: Additional rejection against cosmic muons is achieved from improved timing information and the possibility of constraining the muon to come from the hard scatter vertex. Muon can also be matched to central tracks for improved momentum measurements.

- Central high $p_{T}$ tracks: The information from the silicon tracker improves the impact parameter resolution and momentum resolution for tracks. Isolation from activity in both the tracker and the calorimeter can be required.

- Global variables: The missing transverse energy $E_{T}$ and total transverse energy $H_{T}$ is calculated from the vector and scalar sum of the energy in each calorimeter cell. The precise knowledge of the hard scatter vertex further improves the resolution.

\subsection{Luminosity System}

The event rate $R$ in a collider is proportional to the interaction cross section $\sigma_{\text {int }}$. The factor of proportionality is called the instantaneous luminosity $\mathcal{L}$ :

$$
R=\mathcal{L} \cdot \sigma_{\text {int }}
$$

The instantaneous luminosity depends only on properties of the incoming beams, such as intensity and the time between bunch crossings.

The luminosity detector consists of two arrays of plastic scintillator counters located at $z= \pm 140 \mathrm{~cm}$, as shown Fig. 3.2. Their primary purpose is to determine the instantaneous luminosity at the $\mathrm{D} \varnothing$ interaction region but they are also used for fast calculation of the $z$ position of the interaction vertex in the Level 1 trigger.

The instantaneous luminosity $\mathcal{L}$ is determined from the average number of inelastic collisions per beam crossing $\bar{N}_{L M}$ measured by the luminosity detector

$$
\mathcal{L}=\frac{f \cdot \bar{N}_{L M}}{\sigma_{L M}}
$$


where $f$ is the beam crossing frequency and $\sigma_{L M}$ is the effective cross section for the luminosity detector taking into account the efficiency and acceptance of the detector. The measurement of the luminosity in Run II can be found in Ref. [56].

Equation 3.9 can be integrated over time to get a relation between the number of observed events $N_{o b s}$ and the interaction cross section

$$
N_{o b s}=L \cdot \sigma_{i n t}
$$

where the total luminosity $L$ is related to instantaneous luminosity through

$$
L=\int \mathcal{L} d \mathrm{t} .
$$

In practice the integral is transformed to a sum by assuming that the instantaneous luminosity is constant during a short time interval. The fundamental unit of time for the luminosity measurement is called a luminosity block and each luminosity block is assigned a luminosity block number (LBN) which is monotonically increasing throughout Run II. A luminosity block is typically 60 seconds long, which is short enough that the instantaneous luminosity is effectively constant. 


\section{Chapter 4}

\section{Object Identification}

The top quark decays immediately after it is produced and has to be identified through its decay products. As the decay products traverse the detector, each type of particle will leave a characteristic trace which can be used to identify it. This is illustrated in Fig. 4.1. The process of going from the raw data read out from the detector to reconstructed particles, or collections of particles, is called object identification. This chapter will give an overview of the identification procedure for particles which are important in this thesis.

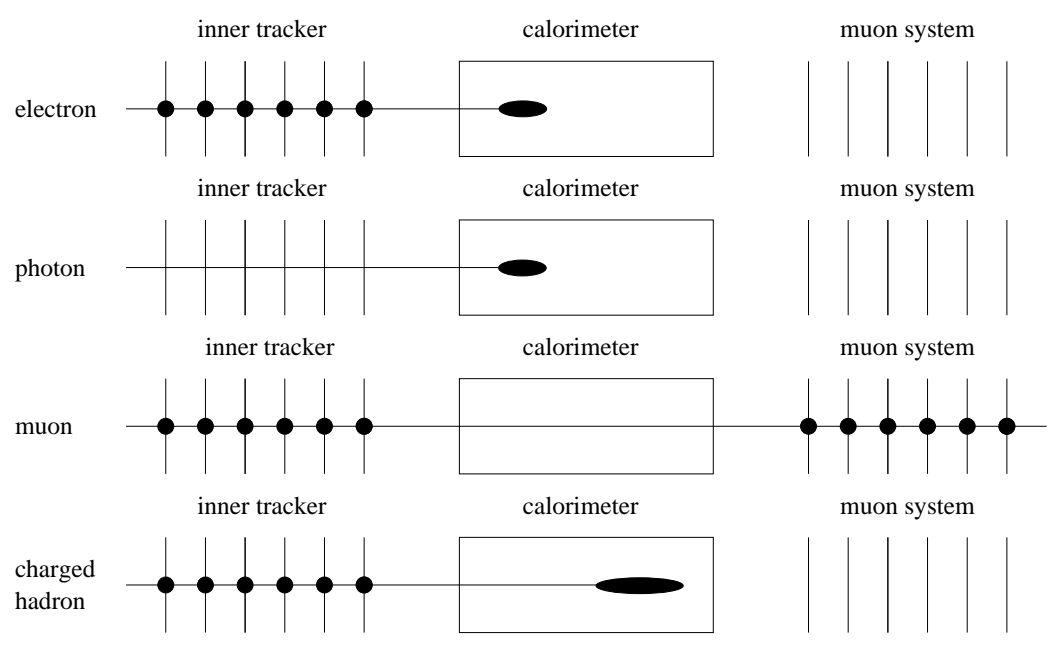

Figure 4.1: Signatures of particles going through the $\mathrm{D} \emptyset$ detector. The charged particles leave hits in the tracking detectors. All particles except muons and neutrinos are stopped in calorimeter where they produce showers. Muons produce hits in the muon detectors outside the calorimeter. 


\subsection{Primary Interaction Point}

The primary interaction point, or primary vertex (PV), has to be reconstructed on an event by event basis. In the $(x, y)$-plane the location is almost constant, and the spread is mainly due to the transverse size of the Tevatron beam which is approximately $30 \mu \mathrm{m}$. In the $z$ direction however, the luminous region extends over more than $60 \mathrm{~cm}^{1}$. Knowledge of the primary vertex position is required to determine directions of physics objects such as jets and muons. Precise knowledge of the primary vertex position is also important when reconstructing secondary decay vertices from long-lived particles.

The primary vertex finding in $\mathrm{D} \varnothing[57,58]$ uses a so called tear-down algorithm. All tracks are fitted together into a single vertex. The $\chi^{2}$ contribution of each single track is then calculated. The track with the highest $\chi^{2}$ contribution is removed and the vertex is re-fitted. This is repeated until the vertex $\chi^{2}$ per degree of freedom is less than 10. After a vertex is found, the whole procedure is repeated with the remaining tracks until all of them have been attached to a vertex.

One event can contain several primary vertices. Most of them are from minimum bias interactions which typically contain few high $p_{T}$ tracks. When several primary vertices are reconstructed, a single one is selected to be the vertex with high momentum transfer, or hard scatter vertex. The average track $p_{T}$ distribution for minimum bias collisions is determined in data. The vertex which has the lowest probability to be a minimum bias interaction is selected as the hard scatter vertex [59].

\subsection{Electrons}

The characteristic signature of electrons is a narrow shower in the electromagnetic part of the calorimeter and in the preshower detectors. Electrons will leave a track in the tracking detectors which can be spatially matched to the shower. Electromagnetic showers without a matching track are classified as photon candidates.

The electromagnetic shower is defined as a cluster of calorimeter cells of radius $\Delta \mathcal{R}<0.4$ around the highest energy tower in the calorimeter. An electron will deposit most of its energy in the first layers of the calorimeter and will thus have a large electromagnetic fraction $f_{E M}=E_{E M} / E_{t o t}$ where $E_{E M}$ is the energy deposited in the electromagnetic layers of the calorimeter and $E_{t o t}$ is the total energy of the cluster. The cluster should also have a longitudinal and lateral shape compatible with the simulated detector response for electrons. Each calorimeter cluster is assigned a $\chi_{C a l}^{2}$ value characterizing how consistent the shower shape is to that of an electron. Electrons tend to have small $\chi_{\text {Cal }}^{2}$ values. Furthermore the cluster is

\footnotetext{
${ }^{1}$ The $z$ position of the primary interaction point follows a Gaussian distribution with a standard deviation of approximately $25 \mathrm{~cm}$.
} 
required to be isolated, meaning that the isolation fraction

$$
f_{\text {iso }}=\frac{E_{\text {tot }}(\Delta \mathcal{R}<0.4)-E_{E M}(\Delta \mathcal{R}<0.2)}{E_{E M}(\Delta \mathcal{R}<0.2)}
$$

should be small. The selection criteria for electrons used in this analysis are [60]:

- $f_{E M}>0.9$.

- $f_{\text {iso }}<0.15$.

- $\chi_{\text {Cal }}^{2}<50$.

- A central track match within $\Delta \phi<0.05$ and $\Delta \eta<0.05$.

After these selections have been applied there is still a large contamination from hadronic jets with high electromagnetic content. To improve the discrimination against jets an electron likelihood discriminant [61] $e_{l h o o d}$ is built for each cluster using six input variables:

- $\chi^{2}$ of the spatial track match between the central track and the calorimeter cluster.

- The ratio of energy measured in the calorimeter and the $p_{T}$ of the central track, $E_{T} / p_{T}$.

- The $\chi_{\text {Cal }}^{2}$ value.

- The electromagnetic fraction $f_{E M}$.

- The impact parameter of the matched central track.

- The distance $\Delta \mathcal{R}$ to the second closest central track from the electromagnetic cluster.

Electron candidates are required to have an electron likelihood discriminant of $e_{\text {lhood }}>0.85$.

\subsection{Muons}

Muons can traverse the entire detector, including the calorimeter, without being stopped. They can be identified with high purity solely from tracks in the muon system. The track in the muon system is required to be matched to a track in the central tracking system. Including the information from the central tracking system allows for a more precise measurement of the muon momentum.

Selection criteria are applied to both the track in the muon system and the matched track in the central tracking system. The following selections are applied to the track in the muon system [62]: 
- At least two hits in the A-layer wire chambers.

- At least one hit in the A-layer scintillators.

- At least two hits in the B- and/or C-layer wire chambers.

- At least one hit in the B- and/or C-layer scintillators.

- The scintillator hit times should be compatible with the beam crossing time window. This selection is used to reject muons from cosmic radiation. The difference between the beam crossing time and the hit in the A-layer scintillators $\left|t_{A}\right|$ is required to be $\left|t_{A}\right|<10 \mathrm{~ns}$. Similarly the time difference for the $\mathrm{B}-$ and C-layer scintillators is required to be $\left|t_{B C}\right|<10 \mathrm{~ns}$.

The track in the central tracking system which is matched to the track in the muon system is required to have the following properties:

- The track fit $\chi^{2} / N_{d o f}<4.0$ to reject badly measured tracks.

- The distance between the muon and the primary vertex in the $z$-direction $\Delta z(\mu, P V)$ is required to be $\Delta z(\mu, P V)<1.0 \mathrm{~cm}$. This selection criterion rejects most of the muons from cosmic radiation.

- The impact parameter significance $d_{c a} / \delta_{d_{c a}}$ should fulfill $d_{c a} / \delta_{d_{c a}}<3.0$. This selection is aimed at removing muons coming from semileptonic decays of $b$ and $c$ quarks.

Muons originating from decays of $W$ bosons are expected to have high $p_{T}$ and to be isolated from other activity in the central tracking system and in the calorimeter. The additional selection criteria for isolated muons are:

- The distance to the closest reconstructed jet $\Delta \mathcal{R}(\mu, j e t)$ is required to be $\Delta \mathcal{R}(\mu, j e t)>0.5$.

- The momentum of all tracks $p_{T}^{\text {trk }}$ in a cone of $\Delta \mathcal{R}=0.5$ around the muon, excluding the muon track, should be small relative to the momentum of the muon

$$
\left(\sum_{\Delta R<0.5} p_{T}^{t r k}\right)<0.06 \cdot p_{T}^{\mu} .
$$

- The energy of all cells $E_{\text {cell }}$ in a hollow cone, shown in Fig. 4.2, around the muon in the calorimeter should be small compared to the momentum of the muon

$$
\left(\sum_{\Delta \mathcal{R}<0.4} E_{\text {cell }}\right)-\left(\sum_{\Delta \mathcal{R}<0.1} E_{\text {cell }}\right)<0.08 \cdot p_{T}^{\mu} .
$$




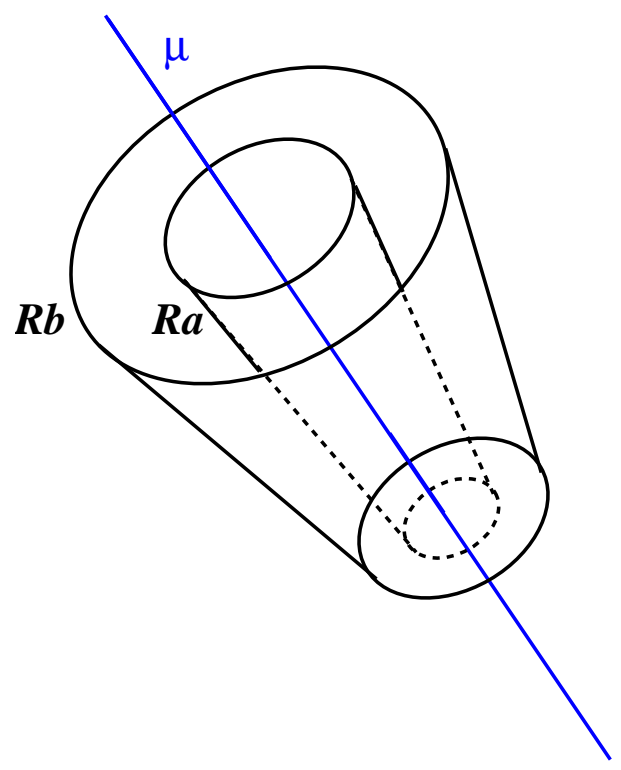

Figure 4.2: A hollow cone in $\Delta \mathcal{R}$, with the inner edge $\Delta \mathcal{R}=R_{a}$ and the outer edge $\Delta \mathcal{R}=R_{b}$ surrounding the muon. In this thesis $R_{a}=0.1$ and $R_{b}=0.4$ are used. The hollow cone surrounding a muon from the decay of a $W$ boson is expected to contain little energy.

\subsection{Jets}

Quarks and gluons are never observed as free particles. Due to their color confinement they hadronize into many colorless particles. These particles are then detected as collimated showers in the tracking system and the calorimeter. Such collimated showers of particles are called jets.

This analysis uses a cone jet algorithm with a cone size of $\Delta \mathcal{R}=0.5[63,64]$. If the cone size is too small, some of the particles from the hadronization may fall outside the cone. However a large cone is not desirable when the final state contains many jets, since the jets are increasingly likely to overlap. The $\mathrm{D} \emptyset$ experiment uses cone sizes of $\Delta \mathcal{R}=0.3,0.5$ or 0.7 .

The energy measured in the calorimeter is not equal to the energy of the particles in the jet after hadronization. The correction factor which brings the measured energy to that of the original particles is called the Jet Energy Scale (JES) [65]. The jet energy scale corrects for the following effects:

- The constant background from the underlying event, the noise from the radioactive decay of the uranium absorber and energy remaining from previous interactions.

- The non-ideal response of the calorimeter due to uninstrumented regions between readout cells, and non-uniformities in the calorimeter response. 
- The difference in response for electromagnetic and nuclear interacting particles $(e / h>1)$ as described in Sec. 3.2.2.

- The fraction of energy which falls outside the jet algorithm cone.

The JES is determined in a sample of events containing a photon and a jet in a back-to-back configuration. The energy scale of electromagnetic objects is known with high precision from $Z \rightarrow e^{+} e^{-}$events. By requiring the momenta of the photon and the jet to be balanced, the JES correction factor can be determined.

The quality selections used to reconstruct and identify jets include:

- $p_{T}>15 \mathrm{GeV} / c$.

- The fraction of energy measured in the coarse hadronic part of the calorimeter $f_{C H}=E_{C H} / E_{t o t}<0.4$.

- The fraction of energy measured in the electromagnetic part of the calorimeter $f_{E M}=E_{E M} / E_{t o t}>0.05$.

- $f_{E M}<0.95$.

- That a fraction of the total energy of the jet, excluding the coarse hadronic part of the calorimeter, is confirmed in the $\mathrm{L} 1$ readout $f_{\mathrm{L} 1}=\frac{E_{\mathrm{L} 1}}{p_{T^{*}\left(1-f_{C H}\right)}}>0.4$.

The last selection is done to remove jets originating from noise in the readout electronics. The new readout electronics for Run II was found to introduce noise which could be reconstructed as false jets. There is however an independent readout chain for the calorimeter used in the L1 trigger decision. This alternative readout does not suffer from the same problem with noise. An efficient way to remove the false jets is to require that a fraction of the energy is read out by both readout chains.

The vector sum of the energy in all the cells which do not fall inside any jet cone in an event is called the unclustered energy.

\subsubsection{Track Based Jets}

Jets can also be reconstructed using the tracking system only. Typically a jet contains several charged particles which can be clustered together into an object called a track-jet. The track-jet algorithm uses a seed track with $p_{T}>1.0 \mathrm{GeV} / c$. It clusters together tracks with $p_{T}>0.5 \mathrm{GeV} / c$ close to the seed using a cone algorithm of radius $\Delta \mathcal{R}=0.5$. Track-jets are used primarily in the reconstruction of secondary vertices (b-tagging), which is described in more detail in Sec. 7.1. To reject poorly reconstructed tracks, all tracks in the track-jet are required to have at least 1 hit in the SMT and a track fit $\chi^{2} / N_{\text {dof }}<4.0$. 


\subsection{Missing Transverse Energy}

Neutrinos rarely interact with matter and therefore escapes detection in the detector. This creates an imbalance in the total transverse momentum of the event pointing in the direction of the escaping neutrino. The missing transverse energy ${ }^{2} \mathbb{E}_{T}$ of the event is calculated from the vector sum of the transverse momenta for all physics objects in the event plus the unclustered energy in the calorimeter.

\footnotetext{
${ }^{2} \mathrm{~A}$ more correct name for the missing transverse energy would be missing transverse momentum, since energy is a scalar quantity. Missing transverse energy is the conventional name and is therefore used throughout this thesis.
} 



\section{Chapter 5}

\section{Sample Definitions}

This chapter defines the samples used in the measurements presented in Papers I and II. Both measurements are performed in the $t \bar{t} \rightarrow \ell+$ jets channel. The final state contains an electron or muon and four jets, out of which two are $b$-jets. Simulated samples of $t \bar{t}$ and background events are used to estimate the efficiency for the event selection.

\subsection{Data Samples}

The $\mathrm{D} \varnothing$ data sample consists of many millions of events which are filtered into dedicated subsamples. The first level of filtering is the so called skimming which is a centralized effort within the $\mathrm{D} \emptyset$ experiment. The skimming splits the $\mathrm{D} \emptyset$ data set into large subsamples. The selection criteria are based on physics signatures such as the existence of an electron or a muon.

The data samples described below are obtained from the skimmed samples by requiring additional selection criteria. The most important sample for the measurements in Papers I and II is the signal sample. Additional samples are selected to derive the performance of the $b$-tagging algorithm and to estimate the multijet background.

\subsubsection{The Preselected Signal Sample}

The $t \bar{t}$ candidate events in data are selected in two steps. First, events are selected based on the expected signature and kinematic properties of $t \bar{t} \rightarrow \ell+$ jets events. In the second step, the $b$-tagging algorithm is applied and events are selected based on the number of $b$-tagged jets they contain. The sample obtained after all kinematic selections, but before $b$-tagging, is called the "preselected sample". Chapter 6 is devoted to a more in-depth description of the preselected sample. 


\subsubsection{The Muon-in-jet Sample}

To derive the efficiency of the $b$-tagging algorithm a sample which is enriched in $b$-jets is needed. To measure an unbiased $b$-tagging efficiency the sample has to be selected without the use of track related information. Jets which contain a muon within the jet cone are enriched in $b$ - and $c$-jets where the quark has decayed semileptonically. The muon-in-jet sample is used to measure the $b$-tagging efficiency for semileptonic $b$ quark decays.

Events in the muon-in-jet sample are required to have at least two jets with $p_{T}>15 \mathrm{GeV} / c$ and $|y|<2.5$. One of the jets is required to have a muon with $p_{T}>8.0 \mathrm{GeV} / c$ within its jet cone, i.e. $\Delta \mathcal{R}(\mu$, jet $)<0.5$.

\subsubsection{The Multijet Data Sample}

A sample of multijet events is needed to determine the probability to accidentally $b$ tag a light-quark jet. This sample is also used when determining the probability for a jet to be misidentified as an electron. The most important selection criterion for the multijet sample is to require that the missing transverse energy is low, $\not_{T}<10 \mathrm{GeV}$. Additional selection criteria for the multijet data sample are:

- One electron candidate, passing the selections: $f_{E M}>0.9, f_{\text {iso }}<0.15$, $\chi_{\text {Cal }}^{2}<50$ and $p_{T}>15 \mathrm{GeV} / c$.

- At least one jet with $p_{T}>15 \mathrm{GeV} / c$.

After these selections the sample is dominated by photon-plus-jet or two-jet events, usually in a back-to-back configuration.

\subsection{Simulated Samples}

Simulated samples of top quark events and background processes are necessary to estimate the efficiency of the event selection. The samples are obtained from event generators which use Monte Carlo techniques [22] to perform the integration of the cross section. This is referred to as Monte Carlo simulation.

The backgrounds to the $t \bar{t}$ signal can be divided into two categories, instrumental and physics backgrounds. The instrumental background events are multijet events which mimic the $t \bar{t}$ signal due to particle misidentification. Misreconstructed events cannot be generated using event generators. A reliable estimate of this background can only come from using real $\mathrm{D} \varnothing$ data. Physics backgrounds are processes where the final state contains isolated high $p_{T}$ leptons in association with jets. The largest physics background is the production of a $W$ boson together with one or more jets. This is referred to as the $W+$ jets background. Other sources of physics backgrounds are small compared to $W+$ jets. 


\begin{tabular}{l|cc}
\hline \hline Generation Parameters & $t \bar{t}$ & $W+$ jets \\
\hline PDF & CTEQ5L & CTEQ5L \\
$Q^{2}$ & $m_{t}^{2}$ & $m_{W}^{2}+\sum p_{T_{j}}^{2}$ \\
Underlying event & Tune A & Tune A \\
$p_{T}$ (parton) & none & $>8 \mathrm{GeV} / c$ \\
$\mid \eta($ parton $) \mid$ & none & $<3.5$ \\
$\Delta R($ parton, parton $)$ & none & $>0.4$ \\
$p_{T}(l)$ & none & none \\
$|\eta(l)|$ & none & $<10$ \\
$p_{T}(\nu)$ & none & none \\
\hline \hline
\end{tabular}

Table 5.1: Main generation parameters for the simulated $t \bar{t}$ and $W+$ jets samples.

All generated events have additional minimum bias $p \bar{p}$ interactions superimposed. The number of added events is taken from a Poisson distribution with a mean which depends on the average instantaneous luminosity.

To simulate the detector response, the generated events are processed through a GEANT [66] simulation of the $\mathrm{D} \varnothing$ detector. The events are then reconstructed using the same software as used for regular detector data.

\subsubsection{The $t \bar{t}$ Sample}

The $t \bar{t}$ signal is generated at $\sqrt{s}=1.96 \mathrm{TeV}$ using ALPGEN 1.3 [67] for the calculation of the hard scatter process and PYTHIA 6.2 [68] for subsequent generation of final state radiation, fragmentation and decay into stable particles. The set of parameters used for the simulation of the underlying event are the so called PyтнIA Tune A $[69,70]$. The parton distribution functions used for modeling the incoming partons are taken from CTEQ5L [25]. No parton-level selections are needed to avoid singularities since the top quarks are very massive. The factorization scale $Q^{2}$ is $m_{t}^{2}$ and the top quark mass is set to $175 \mathrm{GeV} / c^{2}$. Branching fractions and lifetimes for $B$-hadrons are provided by EVTGEN [71]. The $t \bar{t}$ sample contains all $\ell+$ jets final states, including tau leptons. Tau lepton decays are simulated using TAUOLA [72]. The generation parameters are summarized in Tab. 5.1.

\subsubsection{The $W+$ jets Samples}

The $W+$ jets samples are generated with the same generators as the $t \bar{t}$ sample. ALPGEN is used for the hard scatter calculation and PYTHIA for subsequent generation of final state radiation, fragmentation and decay into stable particles. The set of parameters used for the simulation of the underlying event are PYTHIA Tune A. The set of parton distribution functions used are taken from CTEQ5L. The parton-level selection on the lepton is $|\eta|<10$, while the selections for jets are $p_{T}>8 \mathrm{GeV} / c$ 


\begin{tabular}{rr|rr|rr|rr|rr}
\hline \hline$\sigma(\mathrm{pb})$ & $\sigma(\mathrm{pb})$ & $\sigma(\mathrm{pb})$ & \multicolumn{2}{|c|}{$\sigma(\mathrm{pb})$} & \multicolumn{2}{c}{$\sigma(\mathrm{pb})$} \\
\hline \hline$W j$ & 1600 & $W j j$ & 517 & $W j j j$ & 163 & $W j j j j$ & 49.5 & & \\
$W c$ & 51.8 & $W c j$ & 28.6 & $W c j j$ & 19.4 & $W c j j j$ & 3.15 & & \\
& & $W b \bar{b}$ & 9.85 & $W b \bar{b} J$ & 5.24 & $W b \bar{b} J j$ & 2.36 & $W b \bar{b} J j j$ & 0.939 \\
& & $W c \bar{c}$ & 24.3 & $W c \bar{c} J$ & 12.5 & $W c \bar{c} J j$ & 5.83 & $W c \bar{c} J j j$ & 2.36 \\
\hline \hline
\end{tabular}

Table 5.2: The $W$ +jets processes generated with ALPGEN, and their cross sections. $j$ stands for any of $u, d, s, g$ and $J$ is any of $u, d, s, g, c$.

and $|y|<3.5$. The minimum distance between two jets is $\Delta \mathcal{R}\left(j_{1}, j_{2}\right)=0.4$, while no cut is applied on the minimum distance between a jet and the lepton. The factorization scale $Q^{2}$ is $m_{W}^{2}+\sum p_{T_{j}}^{2}$. EVTGEN is used to provide the various branching fractions and lifetimes for $B$-hadrons. Tau leptons are forced to decay leptonically using TAUOLA. The generation parameters are summarized in Tab. 5.1.

\section{Flavor Composition of $W+$ jets}

The analyses in Papers I and II both use $b$-tagging to enhance the $t \bar{t}$ fraction and suppress the $W+$ jets background. The average probability for $W+$ jets events to be $b$-tagged depends crucially on the flavor composition of the jets that are produced in association with the $W$ boson.

To get a reliable estimate of the flavor composition, separate samples are generated for the various combinations of flavored quarks. For $W+4$ jets events, the following combinations of flavored quarks are generated: $W j j j j, W c j j j, W c \bar{c} J j$, and $W b \bar{b} J j$, where $j$ is any of $u, d, s, g$ and $J$ is any of $u, d, s, g, c^{1}$ partons. Table 5.2 lists the generated processes and their cross sections. No parton-level selections are applied on the heavy quarks for the $W b \bar{b}+X$ and $W c \bar{c}+X$ processes.

When generating events in each jet multiplicity bin separately, care has to be taken to avoid double counting of events. An event with $N$ reconstructed jets can be obtained in several ways:

1. $N$ partons are generated in the hard scatter process in ALPGEN, and each of the partons produce a reconstructed jet after hadronization in PYTHIA.

2. $N-1$ partons are generated in ALPGEN, and a hard, large-angle emission of a gluon or quark in the hadronization produces an additional jet. The reconstructed final state contains $N$ jets.

3. $N+1$ partons are generated in the hard scatter process in ALPGEN. One of the partons produce a jet after hadronization in PYTHIA which is either too

\footnotetext{
${ }^{1}$ The $W c \bar{c} c \bar{c}, W b \bar{b} c \bar{c}$, and $W b \bar{b} b \bar{b}$ processes are not included in the model; their cross sections are negligible.
} 
soft to be reconstructed or which falls outside the detector acceptance. The final state again contains $N$ reconstructed jets.

Case 2 and 3 above are properly taken into account in the cross section for case 1. Keeping all three types of events will invalidate the generated cross sections in Tab. 5.2.

To avoid the issue of double counting, a matching scheme is introduced between the partons generated in ALPGEN and the jets from hadronization in PYTHIA. Matching the partons to the reconstructed jets also reduces the theoretical uncertainty introduced by the parton-level selections necessary to avoid singularities from soft and collinear radiation. Schemes which are suitable for this type of matching have been proposed. Two such schemes are the CKKW matching $[73,74]$ and the MLM matching [75]. At the time the samples used in this thesis were generated, the matching schemes were not yet implemented in the event generators. Instead an alternative matching scheme, based on the MLM matching prescription, is used in the analyses in Papers I and II:

1. For all processes except $W b \bar{b}+X$ and $W c \bar{c}+X$, the number of jets is required to be equal to the number of generated partons.

2. For $W b \bar{b}+X$ and $W c \bar{c}+X$, the number of jets is required to be equal to $N-1$ or $N$, with $N$ being the number of generated partons. Events with $N-1$ reconstructed jets are only accepted if all the light-flavor partons are matched to jets. Since there are no parton-level selections applied to the heavy-flavor quarks they can be generated close together and produce only one reconstructed jet. These final states are not included in any of the other generated samples. They are classified as $W(b \bar{b})+X$ or $W(c \bar{c})+X$ where $(b \bar{b})$ or $(c \bar{c})$ indicates that the two heavy-flavor quarks are reconstructed as one jet ${ }^{2}$.

3. All generated light-flavor partons are required to be matched to reconstructed light-flavor jets. No extra jets, which are not matched to a generated parton, are allowed. The exception is the fourth jet multiplicity bin where additional jets are allowed since the fourth jet multiplicity bin is inclusive.

4. Heavy-flavor jets do not have to be matched to a parton for the samples generated without parton-level selections on the heavy quarks. For $W c+X$, the $c$ quark must be generated with parton-level selections. Therefore jetparton matching is required for all partons in this process.

Table 5.3 summarizes all possible flavor configurations consistent with this matching scheme.

\footnotetext{
${ }^{2}$ Events are also classified as $W(b \bar{b})+X$ or $W(c \bar{c})+X$ if the heavy-flavor quarks are generated well separated but only one of the quarks produces a jet which satisfies the $p_{T}$ and $y$ requirements.
} 


\begin{tabular}{c|cccc}
\hline \hline & $W+1$ jet & $W+2$ jets & $W+3$ jets & $W+\geq 4$ jets \\
\hline$W+$ light-flavor jets & $W j$ & $W j j$ & $W j j j$ & $W j j j j$ \\
\hline & & $W b b$ & $W b b J$ & $W b b J j$ \\
& & $W c \bar{c}$ & $W c \bar{c} J$ & $W c \bar{c} J j$ \\
$W+$ heavy-flavor jets & $W(b \bar{b})$ & $W(b \bar{b}) j$ & $W(b \bar{b}) j j$ & $W(b \bar{b}) j j j$ \\
& $W(c \bar{c})$ & $W(c \bar{c}) j$ & $W(c \bar{c}) j j$ & $W(c \bar{c}) j j j$ \\
& $W c$ & $W c j$ & $W c j j$ & $W c j j j$ \\
\hline \hline
\end{tabular}

Table 5.3: Classification of $W+$ jets flavor configurations per jet multiplicity bin. In the above table $j$ is any of $u, d, s, g$ and $J$ is any of $u, d, s, g, c$ partons. $(b \bar{b})$ and $(c \bar{c})$ denote heavy quark pairs reconstructed as one jet.

In each jet multiplicity bin $n$, the fraction of $W+$ jets events in flavor configuration $\Phi$ is obtained from

$$
F_{\Phi, n}=\frac{\sigma_{\Phi, n}^{e f f}}{\sum_{\Phi} \sigma_{\Phi, n}^{e f f}} \quad(n=1,2,3,4)
$$

where the effective cross sections $\sigma_{\Phi, n}^{\text {eff }}$ are derived from the cross sections in Tab. 5.2 taking into account the efficiency of the preselection criteria, trigger efficiency and the parton-jet matching efficiency. The measured flavor composition of $W+$ jets events is summarized in Tab. 5.4. The quoted uncertainties are only due to limited statistics in the generated samples. The effective cross sections and the resulting flavor fractions are also shown in Fig. 5.1. The fractions of $W b \bar{b}, W(b \bar{b}), W c \bar{c}$, $W(c \bar{c})$ increase with jet multiplicity, while the fraction of $W c$ goes down slightly in the fourth jet multiplicity bin.

\begin{tabular}{lcccc}
\hline \hline Contribution & $W+1$ jet & $W+2$ jets & $W+3$ jets & $W+\geq 4$ jets \\
\hline$W b b$ & & $1.04 \pm 0.05$ & $1.78 \pm 0.14$ & $2.70 \pm 0.10$ \\
$W c \bar{c}$ & & $1.44 \pm 0.08$ & $2.71 \pm 0.25$ & $4.37 \pm 0.18$ \\
$W(b \bar{b})$ & $0.71 \pm 0.02$ & $1.26 \pm 0.06$ & $1.81 \pm 0.10$ & $2.75 \pm 0.15$ \\
$W(c \bar{c})$ & $1.12 \pm 0.03$ & $1.96 \pm 0.10$ & $2.96 \pm 0.17$ & $4.36 \pm 0.32$ \\
$W c$ & $4.46 \pm 0.13$ & $6.23 \pm 0.30$ & $6.28 \pm 0.41$ & $4.24 \pm 0.33$ \\
$W+$ light & $93.71 \pm 0.14$ & $88.07 \pm 0.47$ & $84.46 \pm 0.83$ & $81.58 \pm 0.38$ \\
\hline \hline
\end{tabular}

Table 5.4: $W$ +jets flavor fractions (in \%) in each jet multiplicity bin. Parton-jet matching and preselection are required. The quoted uncertainties result only from limited MC statistics.

The matching scheme used in the analyses in Papers I and II does not correspond exactly to the MLM matching scheme. The flavor fractions shown in Tab. 5.4 have been compared to the flavor fractions obtained in low statistics $W+$ jets samples 

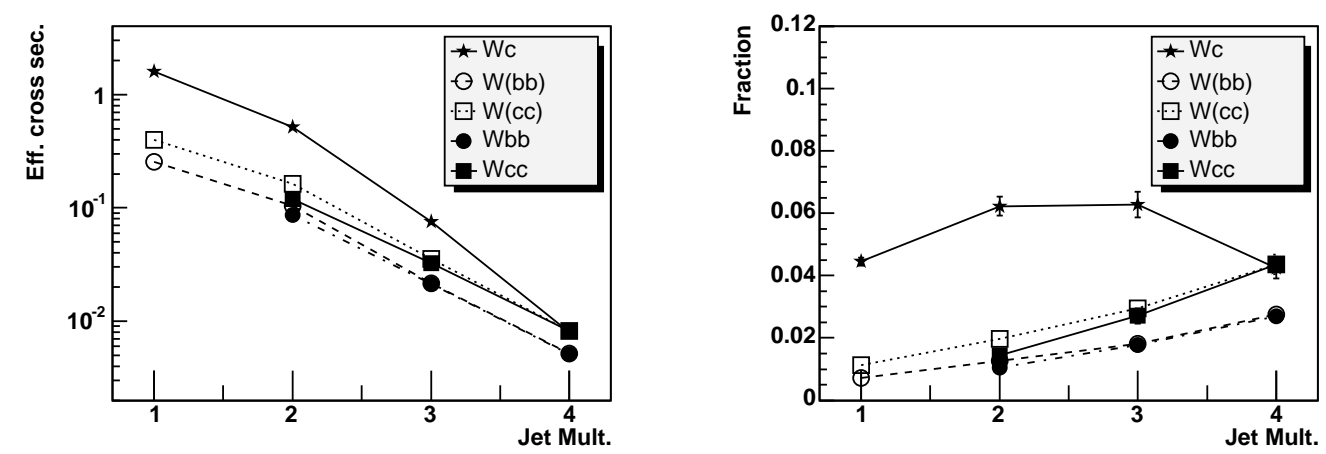

Figure 5.1: Effective cross sections (left) and fractions (right) for the different $W+$ jets processes.

where the MLM matching scheme had been applied. The MLM matching can be applied with different choices of minimum momenta $p_{T}^{m i n}$ of the partons and different values for the maximum distance $\Delta \mathcal{R}^{\max }$ allowed between the parton and the jet. Figure 5.2 shows the ratio of the flavor fractions in Tab. 5.4 to the flavor fractions obtained in the MLM-matched samples for four sets of $\left(p_{T}^{\min }, \Delta \mathcal{R}^{\max }\right)$ choices. The difference is found to be within $20 \%$ and independent of the choice of $\left(p_{T}^{\text {min }}, \Delta \mathcal{R}^{\text {max }}\right)$ for the MLM matching.

The cross sections calculated in ALPGEN, shown in Tab. 5.2, are leading order (LO) cross sections which have large theoretical uncertainties. There has been significant progress recently in the calculation of $W+2$ jets processes at next-to-leading order (NLO) [76, 77]. Special studies for the Tevatron [78] have compared the $W b \bar{b}$ and the $W j j$ cross sections at LO and NLO. The ratio of the $W b \bar{b}$ cross section to the $W j j$ cross section at NLO was found to be a factor of $K=1.05$ higher compared to the LO prediction. This $K$-factor is used to correct the measured fractions of $W b \bar{b}, W(b \bar{b}), W c \bar{c}$ and $W(c \bar{c})$. The fraction of $W c$ is not changed. The fraction of $W$ +light is adjusted so that the sum of all fractions after the correction equals one.

\subsubsection{Other Physics Backgrounds}

There are several physics processes besides $W+$ jets with isolated high $p_{T}$ leptons plus one or more jets in the final state. The production of $Z$ bosons in association with jets has the largest cross section after $W+$ jets production. Since there is no source of $E_{T}$ in $Z+$ jets events, most of them will be rejected by the selection criteria for $t \bar{t}$ events. Since the flavor configuration of the jets in the $Z+$ jets events is expected to be the same as in $W+$ jets events, the expected background from $Z+$ jets events can be absorbed into the background calculation for $W+$ jets.

Some physics backgrounds are expected to have an enhanced fraction of jets 

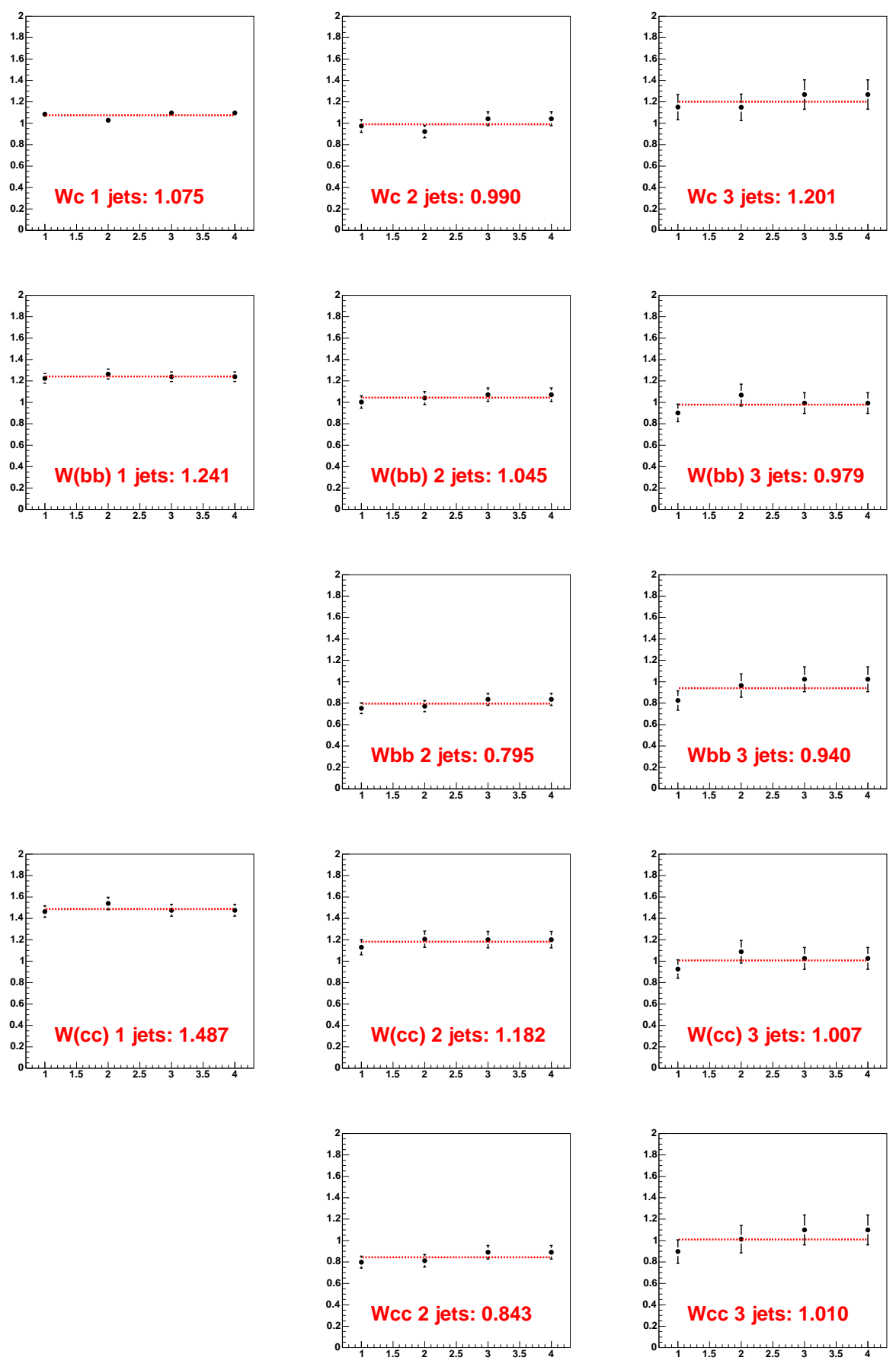

Figure 5.2: Ratios of flavor fractions in Tab. 5.4 to the flavor fractions obtained in low statistics MLM-matched samples. Left, center and right columns correspond to $N_{\text {jets }}=1,2,3$, respectively. In each plot, each of the four points corresponds to one choice of MLM matching: $\left(p_{T}^{\min }, \Delta \mathcal{R}^{\max }\right)=(5,0.5),(5,0.7),(10,0.5),(10,0.7)$. 


\begin{tabular}{lc||c|c}
\hline \hline \multicolumn{1}{lc||}{ process } & \multicolumn{1}{c||}{$\sigma(\mathrm{pb})$} & \multicolumn{2}{c}{ Branching ratio } \\
\hline & & $e$ & $\mu$ \\
\hline \hline$t b \rightarrow \ell \nu b b$ & 0.88 & 0.1259 & 0.1253 \\
$t b q \rightarrow \ell \nu b b j$ & 1.98 & 0.1259 & 0.1253 \\
$W W \rightarrow \ell \nu j j$ & 2.67 & 0.3928 & 0.3912 \\
$W Z \rightarrow \ell \nu j j$ & 0.82 & 0.3928 & 0.3912 \\
$W Z \rightarrow j j \ell \ell$ & 0.24 & 0.4417 & 0.4390 \\
$Z Z \rightarrow \ell \ell j j$ & 0.20 & 0.4417 & 0.4390 \\
$Z / \gamma^{*} \rightarrow \tau \tau$ & 253 & 0.3250 & 0.3171 \\
\hline \hline
\end{tabular}

Table 5.5: Theoretical cross sections for physics backgrounds other than $W+$ jets. All cross sections are at NLO except for $Z / \gamma^{*} \rightarrow \tau \tau$ which is at NNLO [80].

from heavy-flavor $(b, c)$ quarks compared to $W+$ jets. Although their production cross sections are small, these physics backgrounds can contribute noticeably to the expected number of background events after $b$-tagging has been applied. These physics backgrounds are referred to as "other physics backgrounds" in this thesis. The considered processes are $W W, W Z, Z Z, Z \rightarrow \tau \tau$ and single top production.

The diboson $W W, W Z$ and $Z Z$ backgrounds are evaluated using samples generated with ALPGEN interfaced to PYTHIA. The cross sections provided by ALPGEN are the $\mathrm{LO}$ cross sections. Correction factors for the difference between the LO and the NLO cross sections [79] are used.

Single top quark production is treated as a background to the $t \bar{t}$ signal. Samples of single top events are generated with CompHEP [81] interfaced to PYTHIA. Separate samples are generated for the s-channel and the t-channel production. The s-channel is referred to as $t b$ and the t-channel as $t b q$.

To evaluate the $Z / \gamma^{*} \rightarrow \tau \tau$ background, samples generated with PYTHIA containing inclusive $\tau$ lepton decays are used.

Table 5.5 summarizes the generated background processes other than $W+$ jets. The corresponding cross sections are also shown. All cross sections are at NLO, except for $Z / \gamma^{*} \rightarrow \tau \tau$ where the NNLO cross section [80] corresponding to the mass range $60<m_{Z}<130 \mathrm{GeV} / c^{2}$ is used. 



\section{Chapter 6}

\section{The Preselected Signal Sample}

The production cross section for $t \bar{t}$ pairs at the Tevatron is very small compared to the total hard scatter cross section, as was shown in Fig. 2.3. Still, the decays of two such heavy objects as top quarks produce very characteristic final states. In this thesis the $\ell+$ jets final states are studied,

$$
\begin{array}{r}
t \bar{t} \rightarrow W^{+} b \quad W^{-\bar{b}} \\
\hookrightarrow q \bar{q}^{\prime} \hookrightarrow \ell \bar{\nu}_{\ell}
\end{array}
$$

where $\ell=e, \mu$. They are called the $e+$ jets and $\mu+$ jets channel respectively. The $\ell+$ jets topology is characterized by:

- An isolated high $p_{T}$ electron or muon.

- Large missing transverse energy $\left(\mathbb{E}_{T}\right)$ from the undetected neutrino in the $W$ boson decay.

- Four jets (out of which two are $b$-jets).

Requiring a high $p_{T}$ isolated lepton and large $\not_{T}$ removes most of the multijet background events. Some processes, most notably $W+$ jets production, has the same isolated lepton plus $E_{T}$ signature as $t \bar{t} \rightarrow \ell+$ jets. A very efficient way to suppress this topologically irreducible background is to identify one or more of the jets as $b$-jets. This chapter describes the signal sample after all topological selections, but before $b$-tagging has been applied. This sample is called the preselected signal sample. The $b$-tagging algorithm is described in Chapter 7 and the final sample after $b$-tagging has been applied is described in Chapter 8 .

\subsection{Trigger Selection and Luminosity}

The analyses in Papers I and II use data recorded between June 2002 and March 2004. Only the data marked as good by the detector experts is used. The total 


\begin{tabular}{ccc}
\hline & $e+$ jets & $\mu+$ jets \\
\hline $\int \mathcal{L} \mathrm{d} t$ & $226 \mathrm{pb}^{-1}$ & $229 \mathrm{pb}^{-1}$ \\
\hline
\end{tabular}

Table 6.1: Integrated luminosity for the $e+$ jets and $\mu+$ jets channels.

integrated luminosity is given in Tab. 6.1. The difference in luminosity between the $e+$ jets and $\mu+$ jets channels is due to the use of different signal triggers. The signal triggers consist of a lepton requirement and a jet requirement:

- The $e+$ jets signal trigger relies only on calorimeter information. At Level 1 it requires one EM trigger tower with $p_{T}>10 \mathrm{GeV} / c$ and one additional $\mathrm{EM}+\mathrm{H}$ trigger tower with $p_{T}>5 \mathrm{GeV} / c$. At Level 2 the trigger requires one EM object with electromagnetic fraction $f_{E M}>0.85$ and the $p_{T}$ threshold of the $\mathrm{EM}+\mathrm{H}$ trigger tower is raised to $10 \mathrm{GeV} / c$. At Level 3, an EM particle with transverse momentum above $15 \mathrm{GeV} / c$ and a jet with $p_{T}>15 \mathrm{GeV} / c$ are required.

- The $\mu+$ jets signal trigger uses muon information at Level 1 and Level 2, and calorimeter information at Level 1,2 and 3. At Level 1, the trigger requires one muon object based on scintillator hits, and one calorimeter trigger tower with $p_{T}>5 \mathrm{GeV} / c$. There is no momentum selection applied to the muon. At Level 2 the quality requirement on the muon is tightened by requiring hits in the muon wire chambers. The $p_{T}$ threshold for the calorimeter trigger tower is raised to $10 \mathrm{GeV} / c$. The Level 3 part of the trigger requires a jet with $p_{T}>20 \mathrm{GeV} / c$.

\subsection{Selection Criteria}

The preselection criteria are designed to select events with leptonically decaying $W$ bosons and to suppress multijet events. Most of the selection criteria concern the lepton identification or missing transverse energy. A well reconstructed primary interaction vertex is also required to allow for $b$-tagging. All events with at least one reconstructed jet are selected. However events are treated separately throughout the analysis depending on the number of jets they contain. Four separate jet multiplicity bins are considered: events with one, two, three and four or more jets. The $t \bar{t}$ events are expected to be reconstructed mostly in the last two jet multiplicity bins, while the first two jet multiplicity bins are dominated by background processes. Performing the full analysis also in the first two jet multiplicity bins provides a valuable test of the validity of the background prediction.

The selection criteria are described in Sec. 6.2.1, Sec. 6.2.2 and Sec. 6.2.3 below. The probability for an event to pass all the selection criteria is referred to as the 
preselection efficiency. More in-depth descriptions of the preselection criteria are given in Refs. [82, 83].

\subsubsection{Common Selection Criteria for the $e+$ jets and the $\mu+$ jets Channels}

Except for the lepton requirements, the preselection criteria are the same in the $e+$ jets channel and in the $\mu+$ jets channel. These common selections are listed below and are numbered $\mathbf{c . X}$ where $\mathbf{c}$ stands for common:

c.1 At least one jet with $p_{T}>15 \mathrm{GeV} / c$ and $|y|<2.5$. All additional jets in the event are subject to the same $p_{T}$ and $y$ requirements.

c.2 Missing transverse energy $\not_{T}>20 \mathrm{GeV}$.

c.3 Primary interaction vertex $z$-position within the fiducial region of the silicon detector, $\left|z_{P V}\right|<60 \mathrm{~cm}$.

c.4 At least 3 tracks attached to the reconstructed primary interaction vertex.

c.5 The lepton track is compatible with the primary interaction vertex $z$-position, $|\Delta z(\ell, P V)|<1 \mathrm{~cm}$.

\subsubsection{Selection Criteria Specific to the $e+$ jets Channel}

The electron is subject to all quality selections described in Sec. 4.2. The electron likelihood discriminant requirement is the final selection applied, since events without this selection are used for background calculations. The sample of events passing all selection criteria, disregarding the electron likelihood discriminant requirement, is called the $e+$ jets loose sample. The events which also pass the final electron likelihood selection make up the $e+$ jets tight preselected sample. The list of selection criteria specific to the $e+$ jets channel is given below. The selections are numbered e.X where e stands for electron:

e.1 One electron with $p_{T}>20 \mathrm{GeV} / c$ and $|\eta|<1.1$. The $\eta$ requirement constrains the electron to be in the central calorimeter.

e.2 The electron is matched to a track in the central tracking system with $p_{T}>10 \mathrm{GeV} / c$.

e.3 Reject events with a second reconstructed electron with $p_{T}>15 \mathrm{GeV} / c$ in the central or end cap calorimeters. This selection ensures orthogonality between the $t \bar{t} \rightarrow e+$ jets sample and the $t \bar{t} \rightarrow e^{+} e^{-}$sample and rejects $Z \rightarrow e^{+} e^{-}$events. 
e.4 Reject events with an isolated muon with $p_{T}>15 \mathrm{GeV} / c$. This selection ensures orthogonality between the $t \bar{t} \rightarrow e+$ jets sample and the $t \bar{t} \rightarrow e \mu$ and $t \bar{t} \rightarrow \mu+$ jets samples.

e.5 The electron satisfies the electron likelihood requirement, $e_{l h o o d}>0.85$.

\subsubsection{Selection Criteria Specific to the $\mu+$ jets Channel}

The muon is required to pass all identification criteria described in Sec. 4.3. In analogue with the $e+$ jets channel, a loose and a tight preselected sample is defined in order to estimate the instrumental background. The selection criterion to go from the loose to the tight sample in the $\mu+$ jets channel is the muon isolation requirement. It is the last selection criterion applied in the $\mu+$ jets preselection. The list of selection criteria specific to the $\mu+$ jets channel is given below. The selections are numbered $\mathbf{m}$.X where $\mathbf{m}$ stands for muon:

m.1 One muon with $p_{T}>20 \mathrm{GeV} / c$ and $|\eta|<2.0$.

m.2 The muon is not reconstructed within a jet, $\Delta \mathcal{R}(\mu$, jet $)>0.5$.

m.3 Reject events with a second isolated muon with $p_{T}>15 \mathrm{GeV} / c$. This selection ensures orthogonality between the $t \bar{t} \rightarrow \mu+$ jets sample and the $t \bar{t} \rightarrow \mu^{+} \mu^{-}$ sample and rejects $Z \rightarrow \mu^{+} \mu^{-}$events.

m.4 Reject events with a reconstructed electron with $p_{T}>15 \mathrm{GeV} / c$ in the central or end cap calorimeters. This selection ensures orthogonality between the $t \bar{t} \rightarrow \mu+$ jets sample and the $t \bar{t} \rightarrow e \mu$ and $t \bar{t} \rightarrow e+$ jets samples.

m.5 The muon satisfies the isolation requirements listed in Sec. 4.3.

\subsection{Matrix Method}

As discussed in Sec. 5.2, the backgrounds to $t \bar{t} \rightarrow \ell+$ jets events can be divided into two main categories:

- Instrumental multijet background: Events with misidentified leptons. Jets can be identified as electrons if they have a high fraction of their energy deposited in the EM layers of the calorimeter. Muons can be produced inside jets, either from semileptonic decays of heavy-flavor quarks or decays of pions and kaons. If the surrounding jet is not reconstructed, the muons can appear isolated.

- Physics backgrounds: Processes with a true high $p_{T}$ isolated lepton. The physics backgrounds considered are $W+$ jets, single top production, $W W, W Z$, $Z Z$ and $Z \rightarrow \tau \tau$. They are discussed in more detail in Secs. 5.2.2 and 5.2.3. 
Multijet events passing the preselection cannot be properly simulated. Instead the background from multijet events has to be estimated from data. This is done using the so called Matrix Method. The Matrix Method requires two samples, a loose sample and a tight sample. The tight sample is a subsample of the loose sample. The selection criterion which differentiates the tight sample from the loose sample is chosen such that the probability to pass is different for events with true isolated leptons, $\varepsilon_{l e p t}$, and events with misidentified leptons, $\varepsilon_{i n s t r}$. The number of events in the loose sample $N_{\text {loose }}$ and in the tight sample $N_{\text {tight }}$ can be written as

$$
\begin{array}{llll}
N_{\text {loose }} & = & N_{\text {loose }}^{\text {lept }}+ & N_{\text {loose }}^{\text {instr }} \\
N_{\text {tight }} & = & \varepsilon_{\text {lept }} N_{\text {loose }}^{\text {lept }}+\varepsilon_{\text {instr }} N_{\text {loose }}^{\text {instr }}
\end{array}
$$

where $N_{\text {loose }}^{\text {lept }}$ and $N_{\text {loose }}^{\text {instr }}$ are the unknown number of events with real isolated leptons and misidentified leptons in the loose sample. If the two probabilities $\varepsilon_{\text {lept }}$ and $\varepsilon_{\text {instr }}$ are known, the set of equations can be solved in terms of $N_{\text {loose }}^{\text {lept }}$ and $N_{\text {loose }}^{\text {instr }}$ :

$$
N_{\text {loose }}^{\text {lept }}=\frac{N_{\text {tight }}-\varepsilon_{\text {instr }} N_{\text {loose }}}{\varepsilon_{\text {lept }}-\varepsilon_{\text {instr }}} \quad \text { and } \quad N_{\text {loose }}^{\text {instr }}=\frac{\varepsilon_{\text {lept }} N_{\text {loose }}-N_{\text {tight }}}{\varepsilon_{\text {lept }}-\varepsilon_{\text {instr }}}
$$

The number of events with real and misidentified leptons in the tight sample can be obtained by multiplying with the corresponding efficiencies:

$$
N_{\text {tight }}^{\text {lept }}=\varepsilon_{\text {lept }} \cdot N_{\text {loose }}^{\text {lept }} \quad \text { and } \quad N_{\text {tight }}^{\text {instr }}=\varepsilon_{\text {instr }} \cdot N_{\text {loose }}^{\text {instr }}
$$

\subsection{Sample Composition}

The number of observed events passing the preselection criteria is given in Tab. 6.2 for the $e+$ jets channel and in Tab. 6.3 for the $\mu+$ jets channel.

\begin{tabular}{lcccc}
\hline \hline & 1 jet & 2 jets & 3 jets & $\geq 4$ jets \\
\hline \hline$N_{e+\text { jets }}^{\text {presel }}$ & 7765 & 2943 & 741 & 212 \\
\hline$N^{\text {lept }}$ & $7307 \pm 120$ & $2570 \pm 71$ & $598 \pm 32$ & $157 \pm 16$ \\
$N^{\text {instr }}$ & $458 \pm 77$ & $373 \pm 43$ & $143 \pm 14$ & $55 \pm 5$ \\
\hline \hline
\end{tabular}

Table 6.2: Observed number of events in the $e+$ jets preselected sample and expected number of events from the multijet background obtained with the Matrix Method.

The expected number of multijet background events is obtained with the Matrix Method. The selection criterion that differentiate the loose from the tight preselected samples, selections e.5 and $\mathbf{m . 5}$, have very different efficiency for events with real isolated leptons and multijet events, as shown in Tab. 6.4. The values of $\varepsilon_{\text {lept }}$ are measured in a sample of $Z \rightarrow e e$ and $Z \rightarrow \mu \mu$ events respectively. The values of 


\begin{tabular}{lcccc}
\hline \hline & 1 jet & 2 jets & 3 jets & $\geq 4$ jets \\
\hline \hline$N_{\mu+\text { jets }}^{\text {presel }}$ & 6289 & 2559 & 624 & 155 \\
\hline$N^{\text {lept }}$ & $6029 \pm 81$ & $2416 \pm 52$ & $576 \pm 26$ & $143 \pm 13$ \\
$N^{\text {instr }}$ & $260 \pm 8$ & $143 \pm 5$ & $48 \pm 3$ & $12 \pm 1$ \\
\hline \hline
\end{tabular}

Table 6.3: Observed number of events in the $\mu+$ jets preselected sample and expected number of events from the multijet background obtained with the Matrix Method.

\begin{tabular}{l|c|c}
\hline \hline & $\varepsilon_{\text {lept }}$ & $\varepsilon_{\text {instr }}$ \\
\hline$e+$ jets channel & $83 \pm 1$ & $15 \pm 3$ \\
$\mu+$ jets channel & $87 \pm 1$ & $8.5 \pm 3$ \\
\hline \hline
\end{tabular}

Table 6.4: Efficiencies $\varepsilon_{\text {lept }}$ and $\varepsilon_{\text {instr }}$ (in \%) for the Matrix Method in the $e+$ jets channel and in the $\mu+$ jets channel.

$\varepsilon_{\text {instr }}$ are measured in events with low $\not_{T}$. These events are selected by requiring all preselection criteria, except c.2 which is replaced with the criterion ${H_{T}}_{T}<10 \mathrm{GeV}$. The values of $\varepsilon_{\text {instr }}$ are extracted from the fraction of events passing the final selection criterion, e.5 or m.5.

Events with real isolated leptons can be classified into three categories: $W+$ jets events, $t \bar{t}$ events and events from other background processes than $W+$ jets and multijet production. The expected number of $W+$ jets events in the preselected sample is not estimated from Monte Carlo simulation. Instead the number $N^{l e p t}$ obtained from the Matrix Method is used as a first estimate of the number of $W+$ jets events in the preselected sample. Estimating the number of $W+$ jets events before $b$ tagging directly from data has the advantage of being independent of the theoretical cross section for $W+$ jets production, which has large uncertainties associated with it. Although the majority of the $N^{l e p t}$ events containing real isolated leptons in the preselected sample are $W+$ jets events, there are also events from processes such as $t \bar{t}$ production, $W W, W Z, Z Z, Z \rightarrow \tau \tau$ and single top production. To get an improved estimate of the number of $W+$ jets events in the preselected sample, $N_{W}^{\text {presel }}$, the expected contributions from the other sources are subtracted from $N^{l e p t}$

$$
N_{W}^{p r e s e l}=N^{l e p t}-N_{t \bar{t}}^{\text {presel }}-\sum N_{i}^{\text {presel }}
$$

where the subscript $i$ is any of $W W, W Z, Z Z, Z \rightarrow \tau \tau$ and single top production. The expected contribution $N_{i}^{\text {presel }}$ for source $i$ is calculated from the theoretical cross section $\sigma_{i}$, the estimated efficiency to pass the preselection criteria $\epsilon_{i}^{\text {presel }}$ and the luminosity

$$
N_{i}^{\text {presel }}=L \cdot \sigma_{i} \cdot \epsilon_{i}^{\text {presel }}
$$




\begin{tabular}{l|cccc}
\hline \hline & 1 jet & 2 jets & 3 jets & $\geq 4$ jets \\
\hline$t \bar{t} \rightarrow \ell \ell$ & $3.49 \pm 0.09$ & $11.35 \pm 0.16$ & $5.94 \pm 0.12$ & $1.61 \pm 0.07$ \\
$t b \rightarrow \ell \nu b b$ & $5.66 \pm 0.11$ & $15.16 \pm 0.17$ & $4.28 \pm 0.10$ & $0.70 \pm 0.04$ \\
$t b q \rightarrow \ell \nu b b j$ & $5.45 \pm 0.11$ & $12.41 \pm 0.16$ & $5.73 \pm 0.11$ & $1.93 \pm 0.07$ \\
$W W \rightarrow \ell \nu j j$ & $6.98 \pm 0.23$ & $12.15 \pm 0.30$ & $1.58 \pm 0.12$ & $0.14 \pm 0.04$ \\
$W Z \rightarrow \ell \nu j j$ & $6.13 \pm 0.21$ & $12.11 \pm 0.30$ & $1.93 \pm 0.13$ & $0.17 \pm 0.04$ \\
$W Z \rightarrow j j \ell \ell$ & $0.889 \pm 0.077$ & $1.45 \pm 0.10$ & $0.68 \pm 0.07$ & $0.08 \pm 0.02$ \\
$Z Z \rightarrow \ell \ell j j$ & $1.13 \pm 0.08$ & $1.75 \pm 0.10$ & $0.75 \pm 0.07$ & $0.15 \pm 0.03$ \\
$Z / \gamma^{*} \rightarrow \tau \tau$ & $0.05 \pm 0.003$ & $0.02 \pm 0.002$ & $0.01 \pm 0.00$ & $0.00 \pm 0.00$ \\
\hline \hline
\end{tabular}

Table 6.5: Preselection efficiencies (in \%) for physics backgrounds other than $W+$ jets in the $e+$ jets channel. The quoted uncertainties are only from limited statistics.

where $i$ is any of the $W W, W Z, Z Z, Z \rightarrow \tau \tau$ or single top production. The cross sections $\sigma_{i}$ are listed in Tab. 5.5. There are large theoretical uncertainties on these cross sections. However the expected number of events from these processes is very small. Therefore the theoretical uncertainty has negligible impact on the accuracy of the $t \bar{t}$ cross section and branching fraction measurements. The preselection efficiencies $\epsilon_{i}^{\text {presel }}$ are estimated from the simulated samples described in Sec. 5.2. Table 6.5 summarizes the measured preselection efficiencies in the e+jets channel, and Tab. 6.6 summarizes the preselection efficiencies obtained in the $\mu+$ jets channel.

The $t \bar{t}$ process is treated separately. The expected number of $t \bar{t}$ events, $N_{t \bar{t}}^{\text {presel }}$, is calculated as

$$
N_{t \bar{t}}^{\text {presel }}=L \cdot \sigma_{t \bar{t}} \cdot \epsilon_{t \bar{t}}^{\text {presel }}
$$

in analogue with Eq. 6.7. The preselection efficiency for $t \bar{t}$ events $\epsilon_{t \bar{t}}^{\text {presel }}$ is evaluated in the simulated $t \bar{t}$ sample. The $t \bar{t}$ preslection efficiency is shown in Tab. 6.7 for the $e+$ jets channel and in Tab. 6.8 for the $\mu+$ jets channel. The $t \bar{t}$ cross section $\sigma_{t \bar{t}}$ is however the quantity to be measured in the analyses in Papers I and II. When interpreting the observed number of $b$-tagged events in terms of a $t \bar{t}$ cross section it is treated as a free parameter in the cross section likelihood. The $t \bar{t}$ cross section likelihood is discussed in more detail in Sec. 9.1.2. It is often useful to assume the theoretical value of the $t \bar{t}$ cross section of approximately $7 \mathrm{pb}$ when calculating the number of expected events. This assumption is used in several instances in this thesis. Care is taken to point out whether the theoretical or the measured cross section is used in each instance. 


\begin{tabular}{l|cccc}
\hline \hline & 1 jet & 2 jets & 3 jets & $\geq 4$ jets \\
\hline$t \bar{t} \rightarrow \ell \ell$ & $2.51 \pm 0.08$ & $9.62 \pm 0.16$ & $5.56 \pm 0.12$ & $1.55 \pm 0.07$ \\
$t b \rightarrow \ell \nu b b$ & $4.59 \pm 0.11$ & $13.87 \pm 0.18$ & $3.81 \pm 0.10$ & $0.670 \pm 0.043$ \\
$t b q \rightarrow \ell \nu b b j$ & $4.46 \pm 0.10$ & $11.10 \pm 0.16$ & $5.36 \pm 0.12$ & $1.73 \pm 0.07$ \\
$W W \rightarrow \ell \nu j j$ & $6.19 \pm 0.22$ & $12.82 \pm 0.31$ & $1.72 \pm 0.12$ & $0.137 \pm 0.034$ \\
$W Z \rightarrow \ell \nu j j$ & $5.79 \pm 0.22$ & $13.90 \pm 0.33$ & $1.97 \pm 0.13$ & $0.249 \pm 0.048$ \\
$W Z \rightarrow j j \ell \ell$ & $2.67 \pm 0.14$ & $6.22 \pm 0.21$ & $1.08 \pm 0.09$ & $0.121 \pm 0.030$ \\
$Z Z \rightarrow \ell \ell j j$ & $2.82 \pm 0.16$ & $6.32 \pm 0.24$ & $1.60 \pm 0.12$ & $0.159 \pm 0.039$ \\
$Z / \gamma^{*} \rightarrow \tau \tau$ & $0.085 \pm 0.004$ & $0.043 \pm 0.003$ & $0.009 \pm 0.001$ & $0.002 \pm 0.001$ \\
\hline \hline
\end{tabular}

Table 6.6: Preselection efficiencies (in \%) for physics backgrounds other than $W+$ jets in the $\mu+$ jets channel. The quoted uncertainties are only from limited statistics.

\begin{tabular}{|c|c|c|c|c|}
\hline & \multicolumn{2}{|c|}{ Marginal efficiency } & \multicolumn{2}{|c|}{ Cumulative efficiency } \\
\hline & 3 jets & $\geq 4$ jets & 3 jets & $\geq 4$ jets \\
\hline Jet multiplicity & $34.54 \pm 0.28$ & $53.16 \pm 0.30$ & $34.54 \pm 0.28$ & $53.16 \pm 0.30$ \\
\hline Electron within $|\eta|<1.1$ & $62.65 \pm 0.49$ & $50.90 \pm 0.41$ & $21.64 \pm 0.24$ & $27.06 \pm 0.26$ \\
\hline Electron $p_{T}>20 \mathrm{GeV} / c$ & $93.04 \pm 0.34$ & $93.40 \pm 0.29$ & $20.13 \pm 0.23$ & $25.27 \pm 0.25$ \\
\hline Electron identification & $78.39 \pm 0.47$ & $82.31 \pm 0.41$ & $15.78 \pm 0.22$ & $20.80 \pm 0.24$ \\
\hline$E_{T}$ selection & $84.85 \pm 0.45$ & $84.42 \pm 0.40$ & $13.39 \pm 0.20$ & $17.56 \pm 0.23$ \\
\hline Second electron veto & $100.00 \pm 0.00$ & $99.98 \pm 0.02$ & $13.39 \pm 0.20$ & $17.56 \pm 0.23$ \\
\hline Isolated muon veto & $99.97 \pm 0.03$ & $99.96 \pm 0.03$ & $13.38 \pm 0.20$ & $17.55 \pm 0.23$ \\
\hline PV selections & $98.65 \pm 0.11$ & $98.91 \pm 0.10$ & $13.20 \pm 0.20$ & $17.36 \pm 0.22$ \\
\hline Trigger efficiency & $92.72 \pm 0.06$ & $92.69 \pm 0.04$ & $12.24 \pm 0.19$ & $16.09 \pm 0.21$ \\
\hline Data-to-sim. corrections & \multicolumn{2}{|c|}{0.882} & $10.80 \pm 0.16$ & $14.19 \pm 0.18$ \\
\hline$\epsilon_{t \bar{t}}^{p r e s e l}$ & \multicolumn{2}{|c|}{-} & $10.80 \pm 0.16$ & $14.19 \pm 0.18$ \\
\hline
\end{tabular}

Table 6.7: Preselection efficiency (in \%) for $t \bar{t}$ events in the $e+$ jets channel. The quoted uncertainties are only from limited statistics. 


\begin{tabular}{|c|c|c|c|c|}
\hline & \multicolumn{2}{|c|}{ exclusive efficiency } & \multicolumn{2}{|c|}{ cumulative efficiency } \\
\hline & 3 jets & $\geq 4$ jets & 3 jets & $\geq 4$ jets \\
\hline Jet multiplicity & $34.38 \pm 0.28$ & $54.93 \pm 0.30$ & $34.38 \pm 0.28$ & $54.93 \pm 0.30$ \\
\hline Muon identification & $51.94 \pm 0.53$ & $46.88 \pm 0.45$ & $17.91 \pm 0.22$ & $25.75 \pm 0.25$ \\
\hline Muon $p_{T}>20 \mathrm{GeV} / c$ & $72.49 \pm 0.53$ & $72.05 \pm 0.42$ & $12.99 \pm 0.20$ & $18.56 \pm 0.23$ \\
\hline$E_{T}$ selection & $89.60 \pm 0.13$ & $89.26 \pm 0.06$ & $11.64 \pm 0.19$ & $16.57 \pm 0.22$ \\
\hline Second muon veto & $99.97 \pm 0.03$ & $99.94 \pm 0.04$ & $11.64 \pm 0.19$ & $16.56 \pm 0.22$ \\
\hline Electron veto & $99.88 \pm 0.06$ & $99.91 \pm 0.04$ & $11.62 \pm 0.19$ & $16.54 \pm 0.22$ \\
\hline PV selections & $98.45 \pm 0.17$ & $97.64 \pm 0.18$ & $11.44 \pm 0.19$ & $16.15 \pm 0.22$ \\
\hline Trigger efficiency & $91.43 \pm 0.12$ & $92.14 \pm 0.06$ & $10.46 \pm 0.17$ & $14.88 \pm 0.20$ \\
\hline Data-to-sim. corrections & \multicolumn{2}{|c|}{0.945} & $9.88 \pm 0.16$ & $14.06 \pm 0.19$ \\
\hline$\epsilon_{t \bar{t}}^{\text {presel }}$ & \multicolumn{2}{|c|}{-} & $9.88 \pm 0.16$ & $14.06 \pm 0.19$ \\
\hline
\end{tabular}

Table 6.8: Preselection efficiency (in \%) for $t \bar{t}$ events in the $\mu+$ jets channel. The quoted uncertainties are only from limited statistics. 



\section{Chapter 7}

\section{Identification of jets from $b$ quarks}

According to the standard model, top quarks decay almost exclusively into $b$ quarks and $W$ bosons. The $b$ quarks will hadronize and form $B$-hadrons which typically travel a few millimeters in the detector before decaying. By reconstructing the decay vertex, a jet can be identified as coming from a $b$ quark. The identification of jets from $b$ quarks is called $b$-tagging. Using $b$-tagging suppresses the large background to the $t \bar{t}$ signal from $W$ bosons produced in association with light-quark jets.

This chapter describes the algorithm used in this thesis to identify $b$-jets as well as the techniques used to measure the efficiency of the algorithm. Since the performance of the track reconstruction is not well modeled in the simulation of the $\mathrm{D} \varnothing$ detector, efficiency parameterizations are derived in data whenever possible. Simulated $b$-jets are used to derive correction factors to the efficiencies measured in data.

\subsection{Secondary Vertex Algorithm}

The secondary vertex tagging (SVT) algorithm consists of three steps:

- Reconstruction and identification of the primary interaction vertex.

- Reconstruction of track-jets.

- Secondary vertex (SV) finding [84] within track-jets.

Details about the reconstruction of the primary vertex and of track-jets are given in Sec. 4.1 and Sec. 4.4 .1 respectively.

The secondary vertex finding algorithm is applied to every reconstructed trackjet. It uses high quality tracks with two or more hits in the silicon detector and track fit $\chi^{2} / N_{\text {dof }}<3.0$ to ensure good impact parameter resolution. In addition the tracks are required to have $p_{T}>1 \mathrm{GeV} / c$ and an impact parameter significance $\left|d_{c a}\right| / \delta_{d_{c a}}>3.5$. The sign of the impact parameter is inferred from the projection of the impact parameter onto the axis of the track-jet. If the projection points in 
the direction of the jet the impact parameter is defined to be positive, as shown in Fig. 7.1.

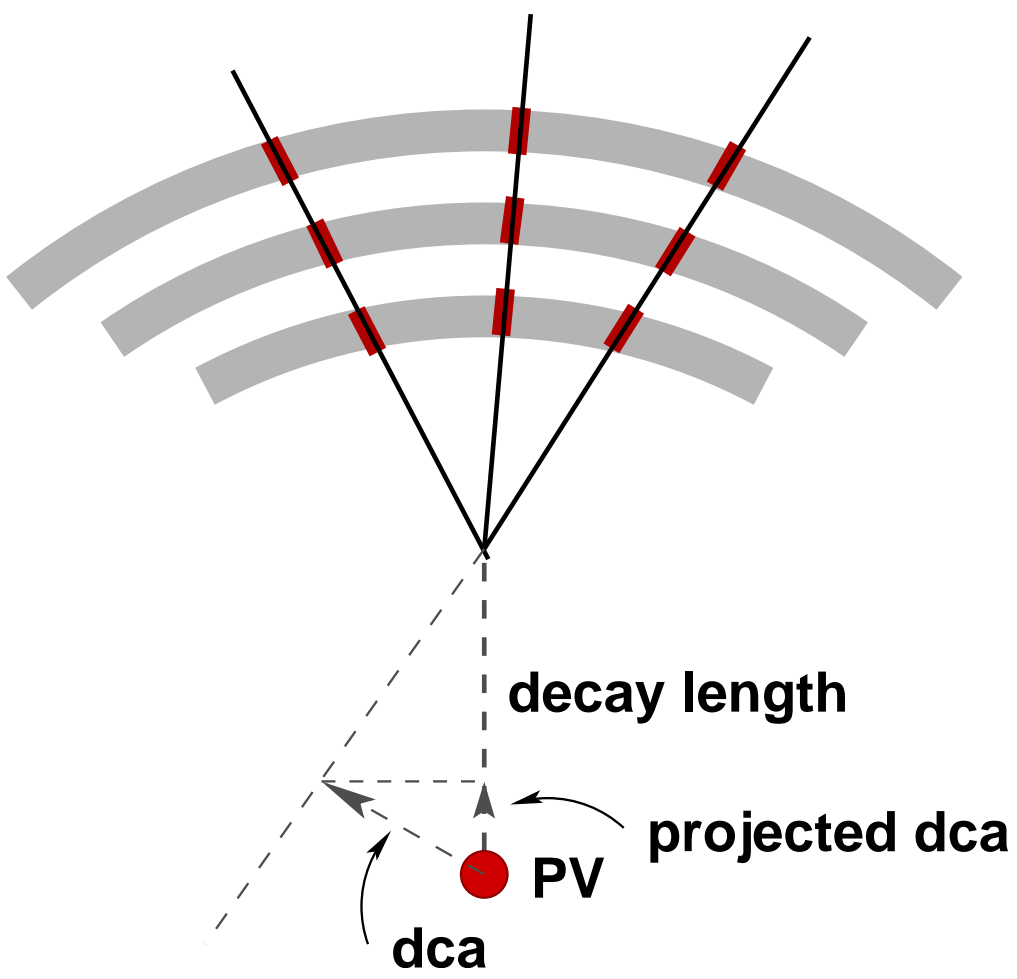

Figure 7.1: Drawing of a secondary vertex. Also shown is the definition of impact parameter $d_{c a}$. The sign of the impact parameter is inferred from the projection of the $d_{c a}$ onto the jet axis. For the track in this drawing, the projection points in the same direction as the jet. This is defined as a positive impact parameter.

The secondary vertices are found using a so called build-up algorithm. It starts by finding seed vertices from the combination of all pairs of tracks within a track-jet. For each seed vertex, the algorithm attaches additional tracks. If the contribution $\Delta \chi^{2}$ to the vertex $\chi^{2}$ from the additional track is $\Delta \chi^{2}<15$, the new vertex with higher multiplicity is kept. This process is repeated until no more tracks can be associated with a vertex. From the resulting list of vertices those that fulfill the following criteria are selected:

- The track multiplicity of the vertex is $\geq 2$.

- The decay length $L_{x y}$, defined as $L_{x y}=\vec{r}_{S V}-\vec{r}_{P V}$, fulfills $\left|L_{x y}\right|<2.6 \mathrm{~cm}$.

- The unsigned decay length significance fulfills $\left|L_{x y}\right| / \delta_{L_{x y}}>7.0$. 
- The collinearity, defined as $\hat{L}_{x y} \cdot \hat{p}_{S V}$ where $\hat{p}_{S V}$ is the normalized vertex momentum vector, is $>0.9$.

- The vertex $\chi^{2} / N_{d o f}<10$.

If the secondary vertex contains only two tracks and the invariant mass of the vertex is consistent with a $K_{S}^{0} \rightarrow \pi^{+} \pi^{-}$decay, a $\Lambda^{0} \rightarrow p^{+} \pi^{-}$decay or a photon conversion $\gamma \rightarrow e^{+} e^{-}$, the vertex is discarded. This procedure is called $V^{0}$ removal and limits the rate at which light-quark jets are mistaken for $b$-jets.

A jet is said to be $b$-tagged if a secondary vertex can be matched to the jet within a cone of radius $\Delta \mathcal{R}=0.5$. If the signed decay length significance of the secondary vertex is less than -7.0 , the jet is called negatively tagged (as opposed to positively tagged, or simply tagged). A negative decay length is assigned to a secondary vertex when the tracks meet at a point behind the primary vertex instead of in front of it, as shown in figure 7.2 .

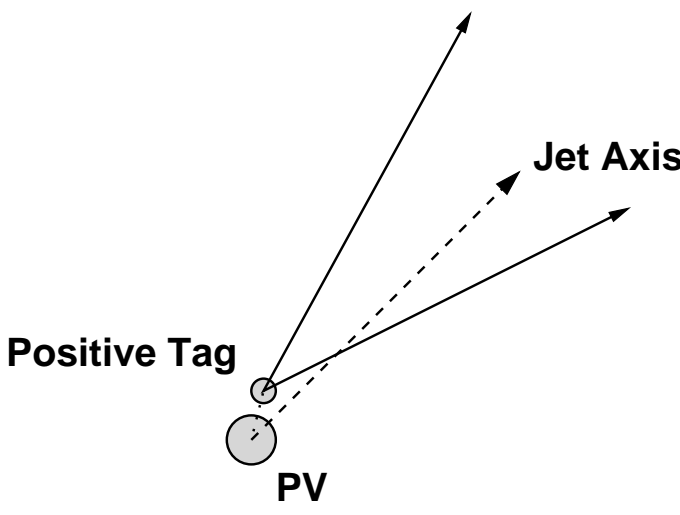

(a) Positive tag.

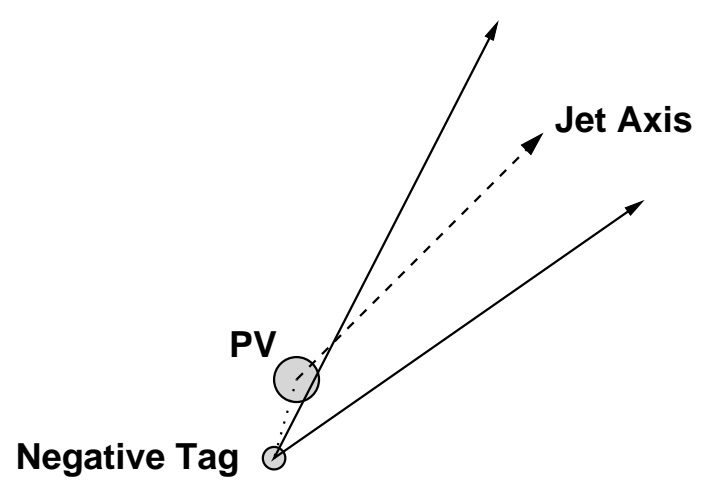

(b) Negative tag.

Figure 7.2: In negative tags the tracks meet behind the primary vertex (labeled PV).

\subsection{Taggability}

The efficiency for the tagging algorithm is broken down into two parts:

- The probability for a jet to have enough high quality tracks reconstructed within the jet cone to allow for the tagging algorithm to be applied. Such a jet is called a taggable jet and the probability is referred to as taggability.

- The probability for a taggable jet to be tagged by the algorithm. This is called tagging efficiency. 
The probability $P_{\text {tagging }}(\alpha)$ for a given jet of flavor $\alpha(b, c$ or light) to be $b$-tagged is the product of the taggability $\epsilon_{\text {taggability }}(\alpha)$ and the tagging efficiency $\epsilon_{\text {tagging }}(\alpha)$ :

$$
P_{\text {tagging }}(\alpha)=\epsilon_{\text {taggability }}(\alpha) \cdot \epsilon_{\text {tagging }}(\alpha)
$$

The reason to split the tagging probability into taggability and tagging efficiency is to decouple instrumental effects in the tracking detectors and the calorimeter from the performance of the tagging algorithm. This also allows for the comparison of different tagging algorithms as long as they use the same definition of taggability. This is of importance when tuning the parameters of the tagging algorithms.

A jet is considered taggable if it is matched within $\Delta \mathcal{R} \leq 0.5$ to a track-jet. The probability for a jet to be taggable depends strongly on the number of reconstructed tracks within the jet cone. Since the number of reconstructed tracks is different in data and simulation, this variable cannot be used to parameterize the taggability measured in data. Instead taggability is parameterized as a function of the hadronic jet $p_{T}$, which is correlated with the number of charged particles in the jet. Tracking efficiency and track quality depends on the rapidity of the tracks. Taggability is therefore parametrized as a function of the jet $p_{T}$ and jet $y$ :

$$
\epsilon_{\text {taggability }}\left(p_{T}, y\right)=\frac{N_{\text {taggable }}\left(p_{T}, y\right)}{N_{\text {jets }}\left(p_{T}, y\right)}
$$

If a jet contains a muon within the jet cone, the muon $p_{T}$ is subtracted from the jet $p_{T}$.

The taggability is studied using the preselected signal samples and the multijet data sample described in Sec. 5.1.3. To avoid any bias from the trigger, only events in the multijet sample which have passed one of the signal triggers are used. The multijet sample has higher statistics than the preselected samples, leading to a smaller statistical uncertainty on the parametrization. Although the shape of the taggability parametrization in the multijet sample is similar to those derived in the signal sample it deviates at low values of jet $p_{T}$, as shown in Fig. 7.3. Therefore the parameterizations from the signal samples are used. The difference between the two parameterizations is treated as a systematic uncertainty on the taggability.

Studies of taggability in the earliest D $\varnothing$ data showed that a large fraction of events with high jet multiplicity contained jets made from calorimeter readout noise. Such jets were not matched to any track-jets in the tracker. Including them in the derivation of the taggability parametrization led to an underestimation of the taggability for real jets. The taggability dependence on the jet multiplicity has also been studied in the current data and the effect of noise jets can still be observed, although the magnitude is significantly smaller. The taggability as a function of jet multiplicity is shown as the light gray points in Fig. 7.4. Also shown is the dependence of the taggability as a function of the jet multiplicity if one of the jet quality selections is tightened. As was described in Sec. 4.4, a fraction of the total 

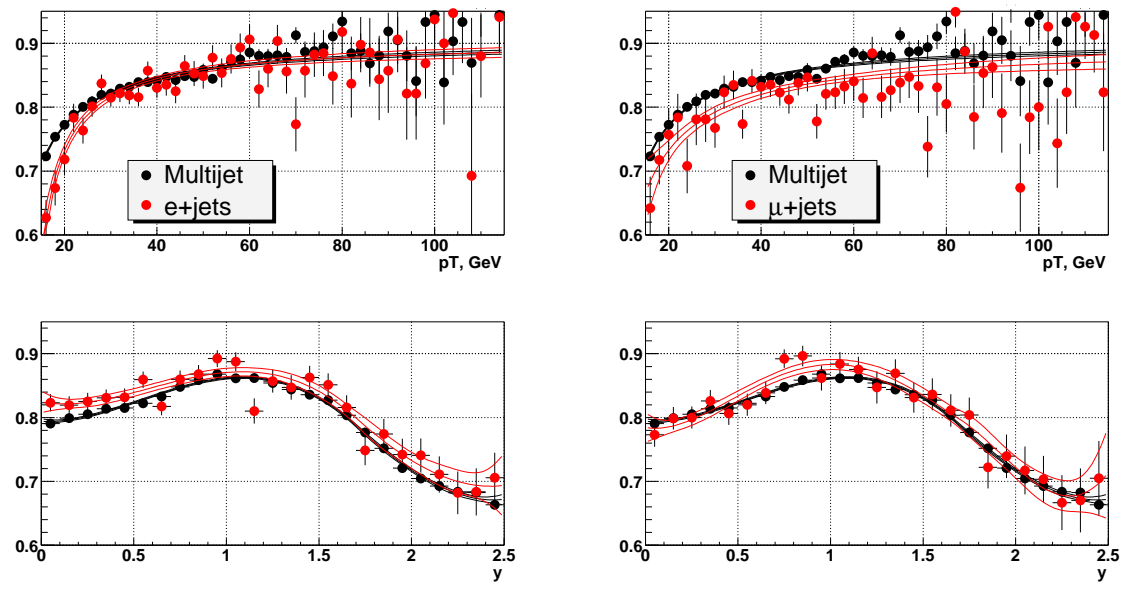

Figure 7.3: Jet taggability as a function of jet $p_{T}$ and $y$ for the first jet multiplicity bin of the preselected $e+$ jets (left) and $\mu+$ jets (right) signal samples as compared to the corresponding taggability in the multijet data sample. The curves indicate the best fit and the one standard deviation error band.

energy of the jet has to be confirmed in the L1 readout. Dark gray and black points display taggability if the fraction of the energy required in the L1 readout is raised to 0.8 (dark gray points) and 1.0 (black points). It is clear that a higher fraction of confirmed energy in L1 readout makes taggability dependence on the jet multiplicity flatter bringing it closer to the expected behavior. Since the standard requirement of 0.4 is used in this thesis, only the first jet multiplicity bin is used to derive the taggability parametrization.

Two-dimensional taggability parameterizations are derived from the onedimensional $p_{T^{-}}$and $y$-dependent parameterizations, assuming the one-dimensional parameterizations are fully uncorrelated. To test the validity of this assumption, the two-dimensional parameterizations are applied to the preselected samples. The agreement between the observed and predicted $p_{T^{-}}$and $y$-distributions of taggable jets is excellent. The distributions are shown in Fig. 7.5 for the $e+$ jets channel and in Fig. 7.6 for the $\mu+$ jets channel. The procedure of applying the parameterizations to the same sample in which they are derived is referred to as a closure test. If the observed and predicted number of taggable jets agree, the assumption that the two one-dimensional parameterizations are uncorrelated is correct.

\subsubsection{Flavor Dependence of Taggability}

The taggability measured in data represents the taggability averaged over all jet flavors. The data sample is dominated by light-flavor jets, the heavy-flavor content in the first jet multiplicity bin is expected to be approximately $1 \%$. The taggability 

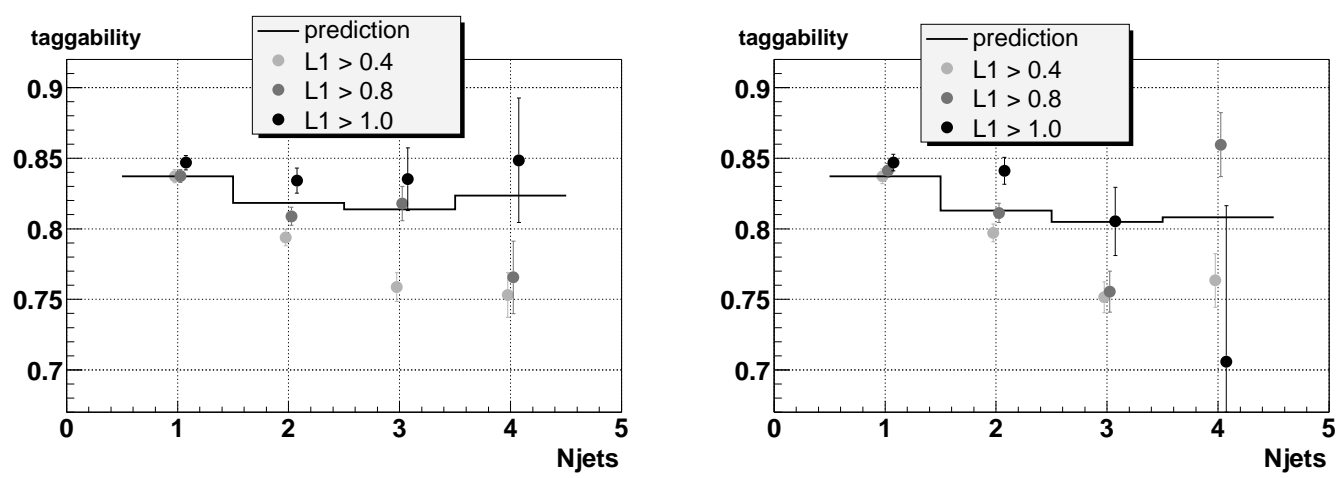

Figure 7.4: Jet taggability as a function of the number of jets in the event for the preselected $e+$ jets (left) and $\mu+$ jets (right) signal samples. Jets passing standard jet quality selections are shown in the light gray points. The dark gray and black points correspond to a higher fraction of the jet energy confirmed in the L1 readout than required for standard jets.
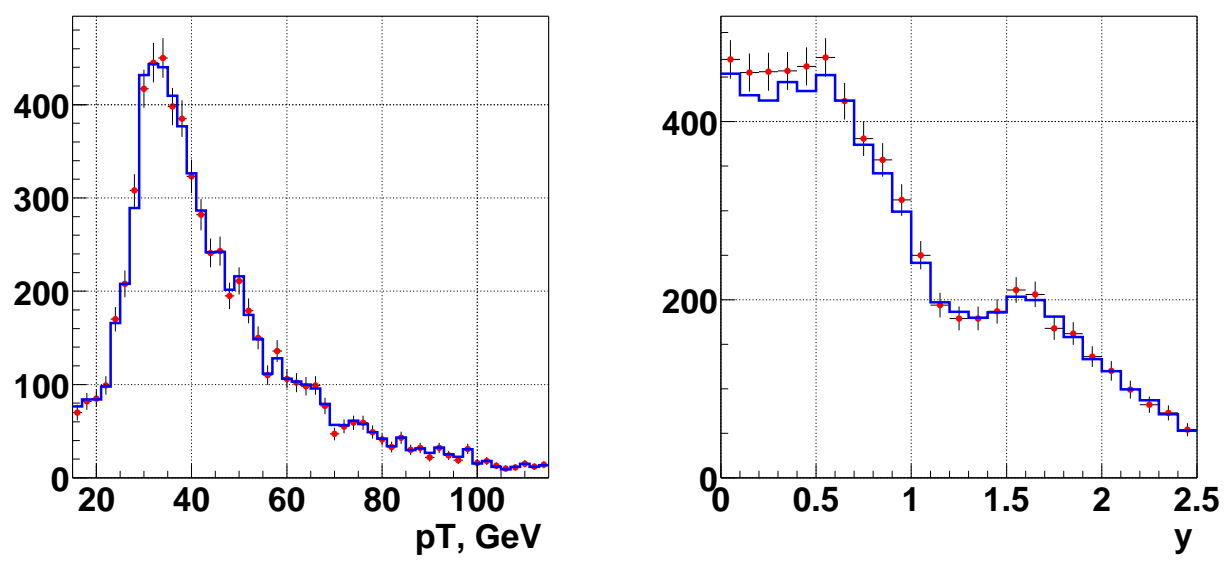

Figure 7.5: Observed jet taggability distributions (points), and distributions predicted by the two-dimensional taggability parameterization (histograms) in the $e+$ jets preselected sample. The left plot shows the distribution as a function of jet $p_{T}$ and right plot shows the distribution as a function of jet $y$. 

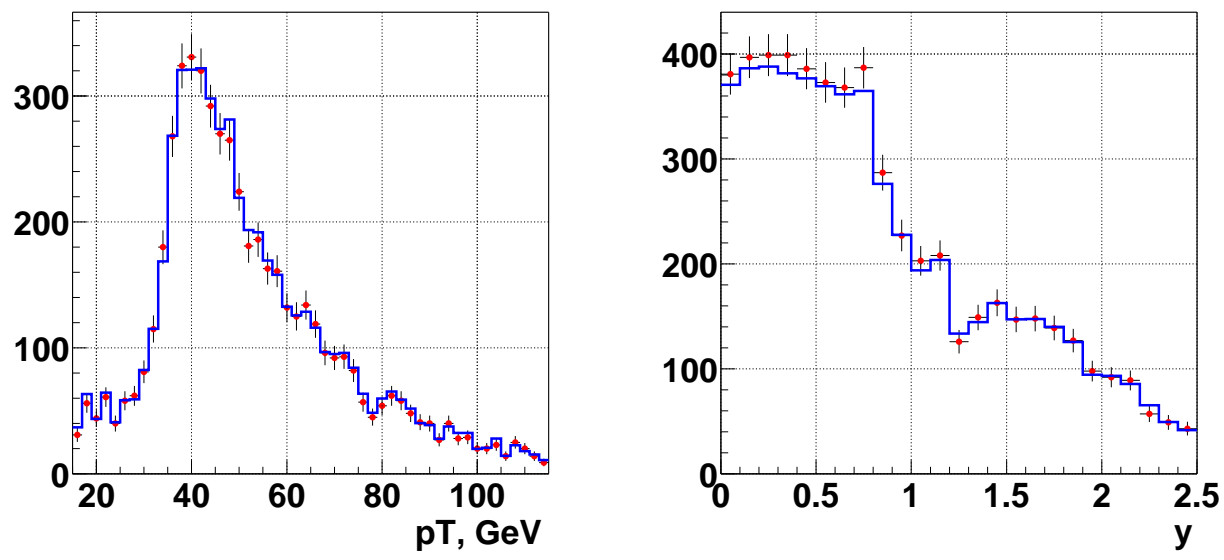

Figure 7.6: Observed jet taggability distributions (points), and distributions predicted by the two-dimensional taggability parameterization (histograms) in the $\mu+$ jets preselected sample. The left plot shows the distribution as a function of jet $p_{T}$ and right plot shows the distribution as a function of jet $y$.

is expected to have some flavor dependence since the track $p_{T}$ spectrum is harder and the average track multiplicity is larger for $b$-jets than for light-flavor jets due to the difference in fragmentation. This is confirmed using simulated $b-, c$ - and light-quark jets.

Figure 7.7 (left) shows the taggability for $b$-, $c$ - and light-quark jets as a function of jet $p_{T}$ and jet $y$, determined in simulated multijet events. The ratios of $b$ - to lightquark and $c$ - to light-quark jet taggabilities are shown in Fig. 7.7 (right). The largest difference in taggability of about $10 \%$ is observed between $b$ - and light-quark jets in the low $p_{T}$ region corresponding to jets with low track multiplicity. The parametrized ratios are used as flavor dependent correction factors to the taggability.

\subsection{Efficiency for Heavy-Flavor Jets}

Measuring the $b$-tagging efficiency in data is difficult without knowing the flavor composition of the jets. Since the Monte Carlo simulation overestimates the efficiency of the SVT algorithm it is still desirable to estimate the $b$-tagging efficiency using only detector data. Information of the flavor content, which is independent of the secondary vertex algorithm, can be obtained for jets containing muons within the jet cone. The muon-in-jet data sample, discussed in Sec. 5.1.2, is therefore used to derive the $b$-tagging efficiency. This sample is enriched in $b$-jets where the $b$ quark has decayed semileptonically,

$$
b \rightarrow c \mu^{-} \nu_{\mu}
$$



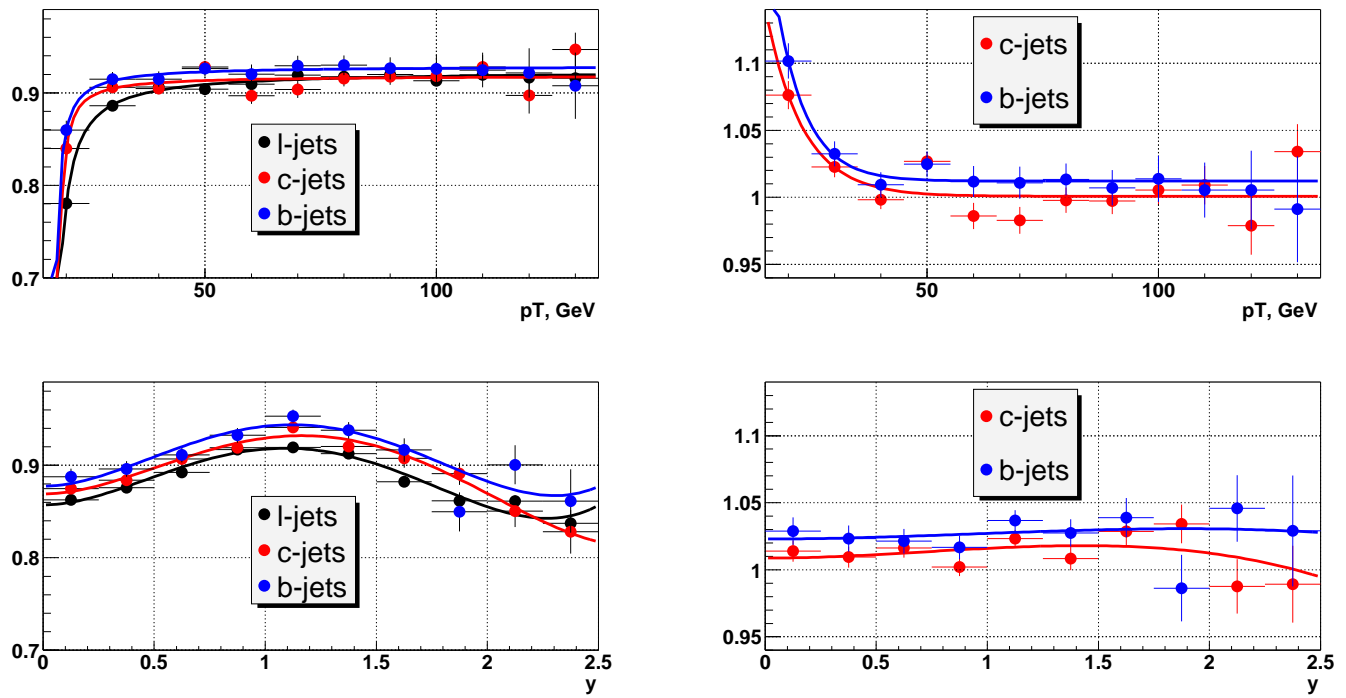

Figure 7.7: The left two plots show the taggability as a function of jet $p_{T}$ and $y$ for $b$-, $c$ - and light-quark jets in simulated multijet events. The right two plots show the ratios of $b$ - to light-quark and $c$ - to light-quark jet taggabilities.

The $b$-tagging efficiency is derived with a method [85] based on the same principles as the Matrix Method. The method considers two samples with different fractions of $b$-jets, called the $n$ and the $p$ sample. The $n$ sample is the sample with smaller fraction of $b$-jets and the $p$ sample is a subset of the $n$ sample. To these two samples, two different and uncorrelated tagging algorithms are applied. The total number of events in the $n$ and the $p$ samples, together with the number of tagged events by the two algorithms is used to write a system of eight equations which can be solved for the $b$-tagging efficiency.

The events in the muon-in-jet sample with at least two taggable jets defines the $n$ sample. The $p$ sample is obtained by requiring that the opposite jet (the jet not containing the muon) is $b$-tagged. The two tagging algorithms used are the SVT algorithm and the muon tagger. The muon tagger tags jets with a muon inside the jet cone with $p_{\text {Trel }}>1 \mathrm{GeV} / c$, where $p_{\text {Trel }}$ is defined as the transverse momentum of the muon relative to the combined muon plus jet axis, shown in Fig. 7.8.

Denoting the events tagged by the SVT and the muon tagger with superscript 


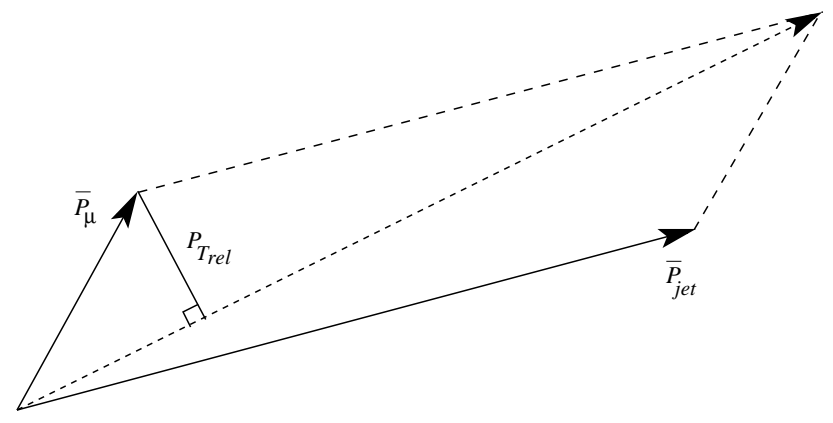

Figure 7.8: Definition of $p_{\text {Trel }}$ for a muon inside a jet.

SVT and $\mu$ respectively, the eight equations can be identified as:

$$
\begin{aligned}
n & =n_{b}+n_{n o n-b} \\
n^{\mu} & =\epsilon_{b}^{\mu} n_{b}+\epsilon_{n o n-b}^{\mu} n_{n o n-b} \\
n^{S V T} & =\epsilon_{b}^{S V T} n_{b}+\epsilon_{n o n-b}^{S V T} n_{n o n-b} \\
n^{\mu, S V T} & =\epsilon_{b}^{\mu} \epsilon_{b}^{S V T} n_{b}+\epsilon_{n o n-b}^{\mu} \epsilon_{n o n-b}^{S V T} n_{n o n-b} \\
p & =p_{b}+p_{\text {non-b }} \\
p^{\mu} & =\epsilon_{b}^{\mu} p_{b}+\epsilon_{\text {non-b }}^{\mu} p_{n o n-b} \\
p^{S V T} & =\epsilon_{b}^{S V T} p_{b}+\epsilon_{n o n-b}^{S V T} p_{n o n-b} \\
p^{\mu, S V T} & =\epsilon_{b}^{\mu} \epsilon_{b}^{S V T} p_{b}+\epsilon_{n o n-b}^{\mu} \epsilon_{n o n-b}^{S V T} p_{n o n-b}
\end{aligned}
$$

The numbers on the left hand side of the equations are those measured in data and the right hand side consists of eight unknown quantities. These are the number of $b$ - and non- $b$ jets in the two samples $\left(n_{b}, n_{n o n-b}, p_{b}, p_{n o n-b}\right)$ and the tagging efficiencies for $b$ and non- $b$ jets for the two tagging algorithms $\left(\epsilon_{b}^{S V T}, \epsilon_{n o n-b}^{S V T}, \epsilon_{b}^{\mu}\right.$, $\left.\epsilon_{n o n-b}^{\mu}\right)$. The quantity of interest is the efficiency for the SVT algorithm to tag a $b$-jet, $\epsilon_{b}^{S V T}$. Similar to the taggability, the $b$-tagging efficiency depends on the $p_{T}$ and $y$ of the jet. The $n$ and $p$ samples are therefore divided further into bins of either $p_{T}$ or $y$. The the system of equations is solved for each subsample to obtain the $b$-tagging efficiency parametrization as a function of either the jet $p_{T}$ or the jet $y$. This is shown in Fig. 7.9 together with the parametrized functions. A combined two-dimensional parameterization is derived assuming that $p_{T}$ and $y$ dependencies are not correlated and can be factorized.

Only the efficiency for semileptonically decaying $b$ quarks, $\epsilon_{b \rightarrow \mu}^{d a t a}\left(p_{T}, y\right)$, can be measured in the muon-in-jet sample. The $b$-tagging efficiency including all possible decays of the $b$ quarks, called the inclusive $b$-tagging efficiency, can only be measured using simulated $b$-jets. The inclusive $b$-tagging efficiency obtained from Monte Carlo simulation is an overestimate of the inclusive $b$-tagging efficiency in data, due to the unrealistic description of the $\mathrm{D} \varnothing$ tracking performance in the simulation. A scale 

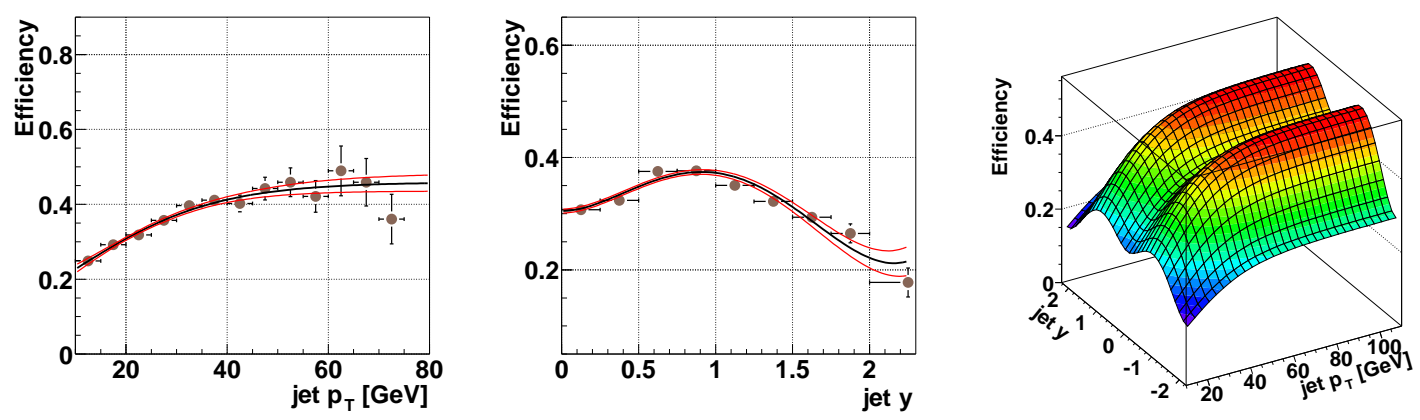

Figure 7.9: Semileptonic $b$-tagging efficiency in data as a function of jet $p_{T}$ (left plot) and jet $y$ (middle plot) measured with the system of eight equations (Eqs. 7.4). Also shown is the best fit and the $\pm 1 \sigma$ uncertainty on the parameterizations. The right plot shows the combined two-dimensional parameterization.

factor is introduced to correct for the difference between data and simulation. The scale factor is defined as:

$$
S F_{b \rightarrow \mu}\left(p_{T}, y\right)=\frac{\epsilon_{b \rightarrow \mu}^{\text {data }}\left(p_{T}, y\right)}{\epsilon_{b \rightarrow \mu}^{\text {sim }}\left(p_{T}, y\right)}
$$

where $\epsilon_{b \rightarrow \mu}^{\text {sim }}$ is the semileptonic $b$-tagging efficiency derived from Monte Carlo simulation. The scale factor for the inclusive $b$-tagging efficiency, $S F_{b}\left(p_{T}, y\right)$, is assumed to be the same as for the semileptonic $b$-tagging efficiency,

$$
S F_{b}\left(p_{T}, y\right)=S F_{b \rightarrow \mu}\left(p_{T}, y\right) .
$$

The $b$-tagging efficiency $\epsilon_{b}\left(p_{T}, y\right)$ can then be written as

$$
\epsilon_{b}\left(p_{T}, y\right)=\epsilon_{b}^{s i m}\left(p_{T}, y\right) \cdot S F_{b}\left(p_{T}, y\right)
$$

where $\epsilon_{b}^{s i m}\left(p_{T}, y\right)$ is the inclusive $b$-tagging efficiency derived from Monte Carlo simulation.

It is not straightforward to measure the $c$-tagging efficiency in data, or the corresponding $c$-tagging scale factor $S F_{c}$. The scale factor is assumed to be equal to the $b$-tagging scale factor,

$$
S F_{c}\left(p_{T}, y\right)=S F_{b}\left(p_{T}, y\right)
$$

and the $c$-tagging efficiency is defined in analogue with the $b$-tagging efficiency:

$$
\epsilon_{c}\left(p_{T}, y\right)=\epsilon_{c}^{s i m}\left(p_{T}, y\right) \cdot S F_{c}\left(p_{T}, y\right)
$$

Evaluating the tagging efficiencies for $b$ - and $c$-jets in the Monte Carlo simulation is straightforward since the jet flavor is known. The efficiency is defined as the ratio 
of the number of tagged jets to the total number of taggable jets of a particular flavor:

$$
\epsilon_{b, c}^{\text {sim }}\left(p_{T}, y\right)=\frac{N_{b, c}^{\text {tagged }}\left(p_{T}, y\right)}{N_{b, c}^{\text {taggable }}\left(p_{T}, y\right)}
$$

The $b$ - and $c$-tagging efficiencies are obtained as functions of jet $p_{T}$ and $y$ and combined into two-dimensional parameterizations assuming that the $p_{T}$ and $y$ dependencies are not correlated and can be factorized.

The inclusive and semileptonic $b$-tagging efficiencies are measured in the simulated $t \bar{t}$ sample. The parameterizations are shown in Fig. 7.10. The inclusive and semileptonic efficiencies are found to be very similar, except in the low $p_{T}$ region where the presence of the muon track increases the tagging efficiency for semileptonic decays. At higher values of jet $p_{T}$, this effect is less important due to the higher track multiplicity. The inclusive $c$-tagging efficiency is shown in Fig. 7.11. The relative statistical uncertainties for all parameterizations are shown in Fig. 7.12.
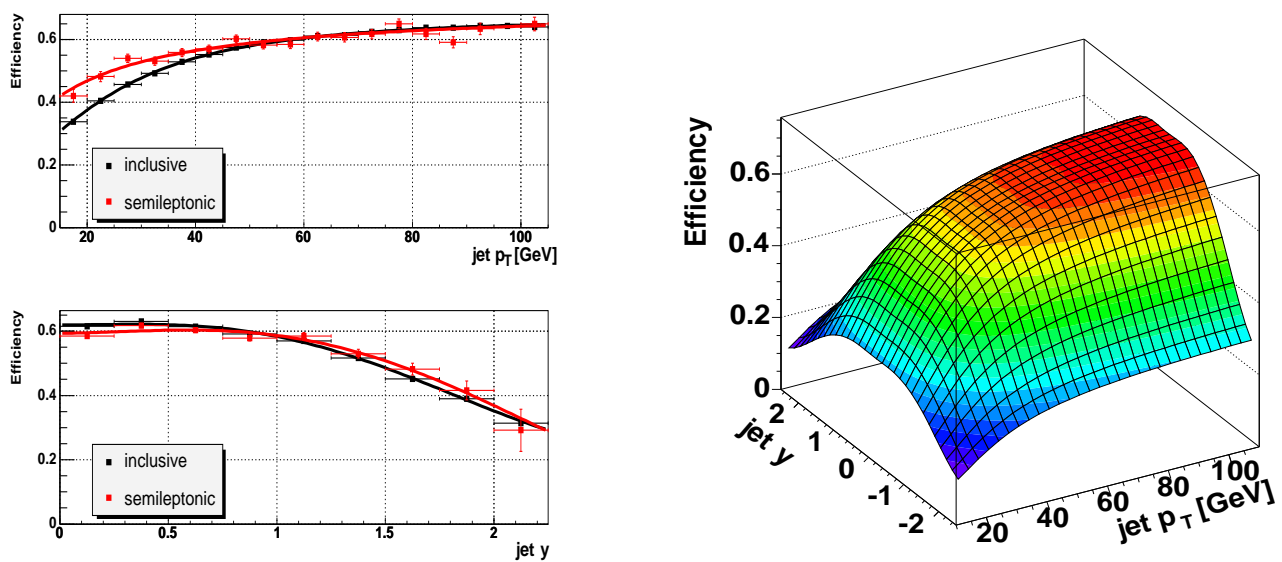

Figure 7.10: The inclusive and semileptonic $b$-tagging efficiencies evaluated in simulated $t \bar{t}$ events as a function of jet $p_{T}$ (upper left) and $y$ (lower left). The right plot shows the combined two-dimensional parametrization for the inclusive $b$-tagging efficiency.

\subsection{Mistag Rate}

A $b$-tagged light-flavor jet (a jet from a $u, d$ or $s$ quark or a gluon) is called a mistagged jet (or mistag). Tracks originating from the primary vertex can appear displaced due to resolution effects in the tracker or due to misreconstruction, the former being the dominant source. The mistag rate due to resolution effects can be evaluated using negative tags, described in Sec. 7.1. 

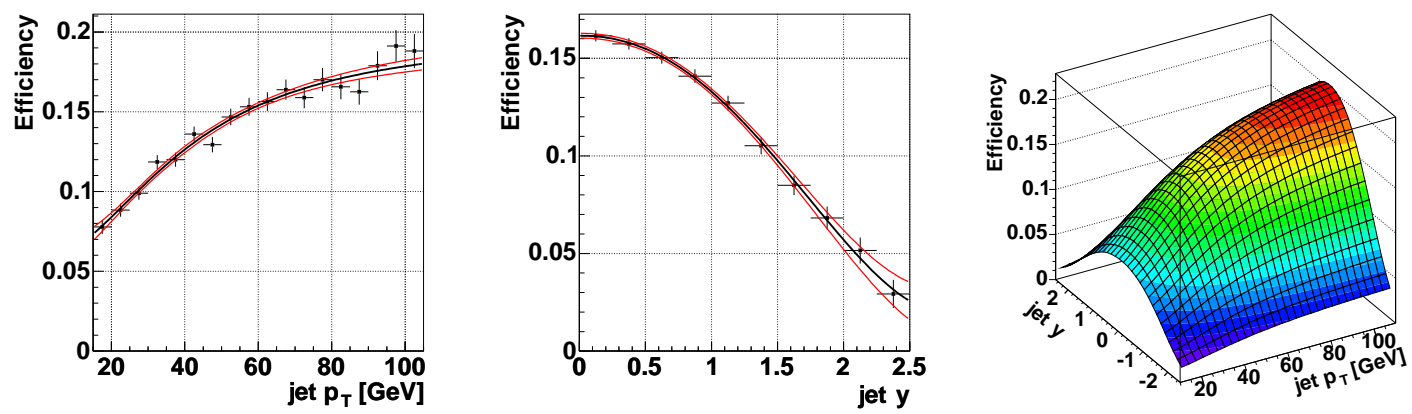

Figure 7.11: The inclusive $c$-tagging efficiency evaluated in simulated $t \bar{t}$ events as a function of jet $p_{T}$ (left plot) and $y$ (middle plot). The right plot shows the combined two-dimensional parametrization.

Resolution effects are expected to contribute equally to both the positive and the negative tag rate. Therefore the negative tag rate in data is a good estimate of the rate at which light-flavor jets are mistagged. The negative tag rate measured in data $\epsilon_{\text {neg }}^{\text {data }}\left(p_{T}, y\right)$ for taggable jets is defined as

$$
\epsilon_{n e g}^{\text {data }}\left(p_{T}, y\right)=\frac{N_{\text {neg tag }}\left(p_{T}, y\right)}{N_{\text {taggable }}\left(p_{T}, y\right)}
$$

and is evaluated in the multijet data sample. Jet triggers can bias the $p_{T}$-spectrum of the jets, and track triggers can bias the measurement of the negative tag rate. To keep the jets unbiased only events passing an electromagnetic object (single EM) trigger without any track requirements are used. The measured negative tag rate is parametrized as a function of jet $p_{T}$ and $y$, as shown in Fig. 7.13. A twodimensional parametrization is obtained from the one-dimensional ones assuming they are uncorrelated.

To investigate any dependence on the choice of trigger, an alternative parametrization is obtained from events in the multijet sample passing the $e+$ jets signal trigger. A comparison of the two parameterizations is shown in Fig. 7.14.

A closure test for the negative tag rate parametrization is performed by applying it to the multijet sample. The observed and predicted number of negatively tagged jets are in good agreement. This is shown in Fig. 7.15 for the inclusive sample and in Fig. 7.16 for each jet multiplicity bin separately. Shown is also the prediction from the alternative parametrization obtained from events passing the $e+$ jets signal trigger. This parametrization shows worse agreement when applied to the sample with the single EM trigger requirement.

As a cross check, the parametrized negative tag rate is also applied to all taggable jets in the preselected signal samples and the prediction is compared to the number of observed negative tags. The result is shown in Tab. 7.1 for the $\mu+$ jets, $e+$ jets and 

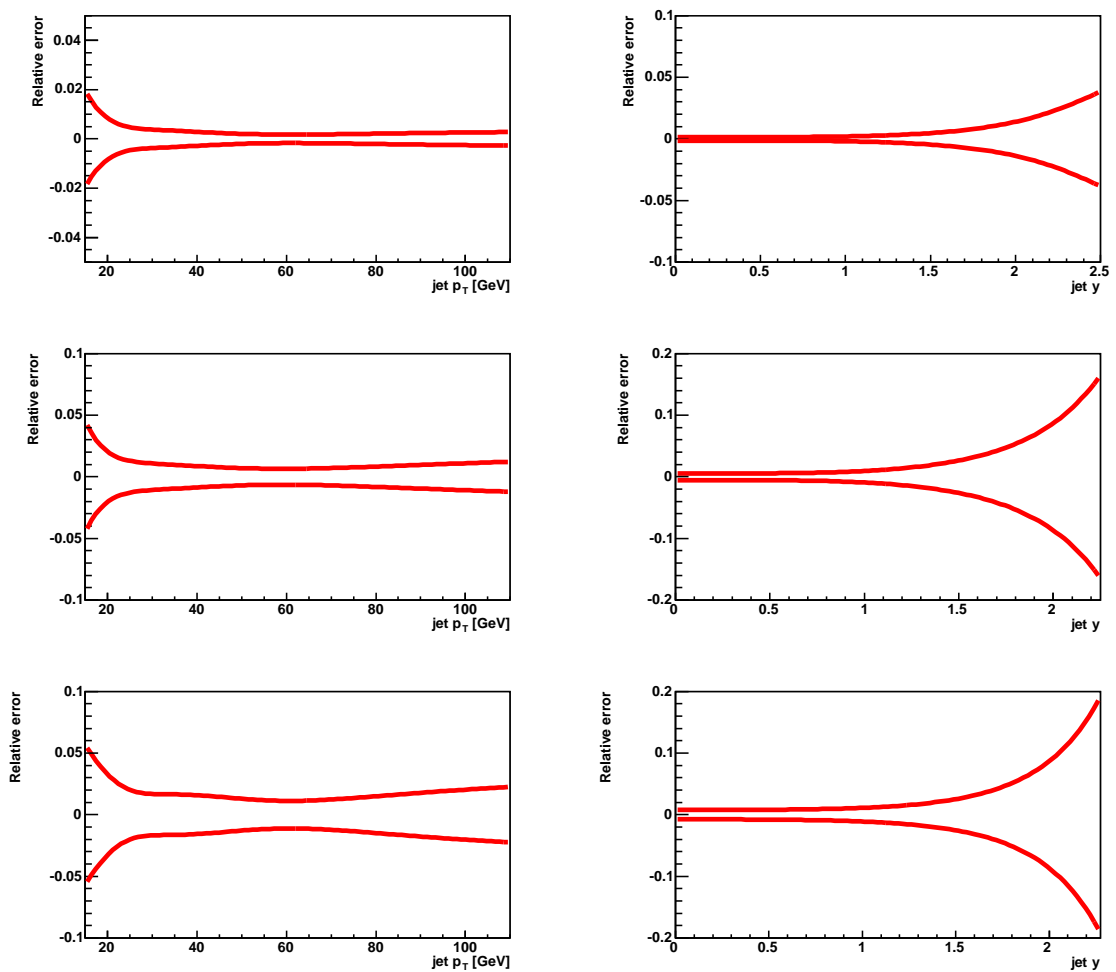

Figure 7.12: The relative statistical uncertainties for the tagging efficiency parameterizations evaluated in simulated $t \bar{t}$ events. The top two plots show the relative uncertainties for the inclusive $b$-tagging efficiency as a function of jet $p_{T}$ (left plot) and $y$ (right plot). The middle two plots are for the semileptonic $b$-tagging efficiency and the lower two plots are for the inclusive $c$-tagging efficiency.

combined $\ell+$ jets channels. Figure 7.17 shows the agreement in the inclusive $\ell+$ jets sample and Fig. 7.18 for each jet multiplicity bin separately. All plots show good agreement between the observed and predicted number of negative tags. No significant difference can be observed from using the negative tag rate parametrization derived with the $e+$ jets signal trigger requirement.

\subsubsection{Correction Factors}

There are two major effects which needs to be taken into account before the negative tag rate equals the mistag rate:

- Heavy-flavor contamination: The jets used to determine the negative tag rate in the multijet sample are not all light-flavor jets. Jets from heavy-flavor quarks have a higher negative tag rate than light-flavor jets. The displaced tracks from the decay of the $D$ - or $B$-hadron increase the probability to form 

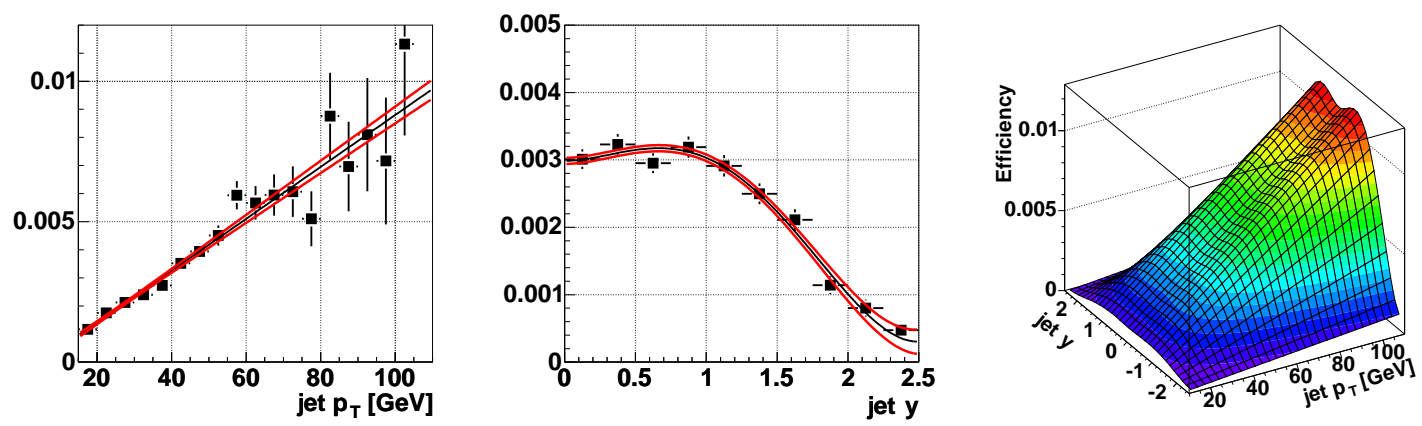

Figure 7.13: The negative tag rate as a function of jet $p_{T}$ (left plot) and jet $y$ (middle plot). The right plot shows the two-dimensional parametrization.

a negative tag. The negative tag rate measured in the multijet sample is an overestimate of the magnitude of resolution effects due to the contamination from heavy-flavor jets. To compensate for the heavy-flavor contamination, a correction factor $S F_{h f}$ is introduced.

- Additional mistag rate from long-lived particles: Light-flavor jets can be mistagged due to the presence of tracks from $K_{S}^{0}$ or $\Lambda^{0}$ decays and interactions with material, which are not completely removed by the $V^{0}$ filter. The mistag rate of light-flavor jets is therefore underestimated when only resolution effects are considered. To take into account the additional mistag rate due to long-lived particles, a correction factor called $S F_{l l}$ is introduced where $l l$ stands for long-lived.

The two correction factors $S F_{h f}$ and $S F_{l l}$ are obtained from simulated multijet events:

- $S F_{h f}$ is defined the ratio of the negative tag rate for light-flavor jets to the negative tag rate for inclusive jet flavors:

$$
S F_{h f}=\frac{\epsilon_{n e g}^{l i g h t}}{\epsilon_{n e g}}
$$

- $S F_{l l}$ is defined as the ratio of positive to negative tag rate for light-flavor jets:

$$
S F_{l l}=\frac{\epsilon_{p o s}^{l i g h t}}{\epsilon_{n e g}^{l i g h t}}
$$

The two correction factors are measured as functions of jet $p_{T}$ and $y$, and are shown in Fig. 7.19. 

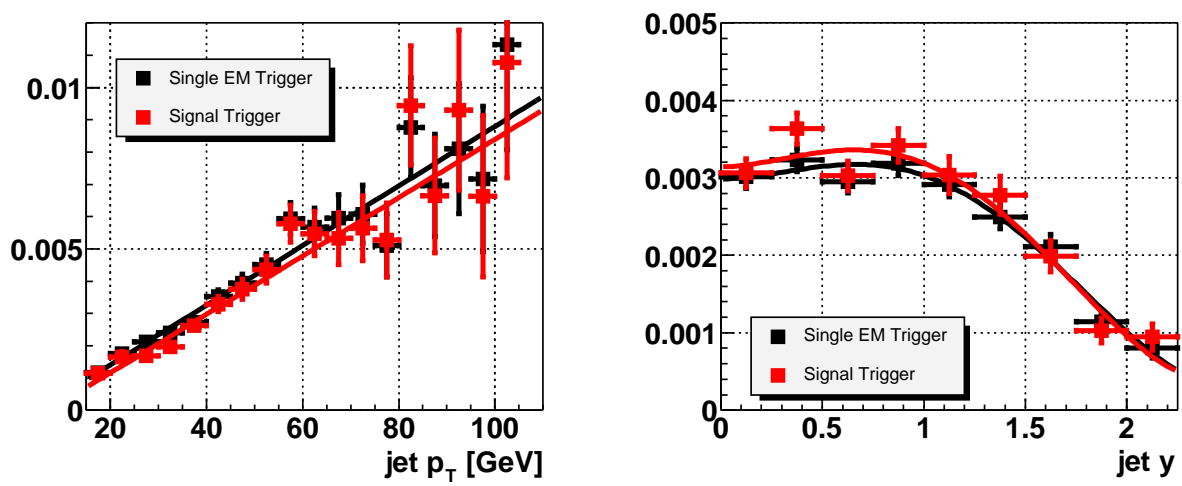

Figure 7.14: A comparison of the default negative tag rate function derived from events passing a single EM trigger and an alternative parametrization obtained from events passing the $e+$ jets signal trigger. The left plot shows the negative tag rate as a function of jet $p_{T}$ and the right plots as a function of jet $y$.

The product of the negative tag rate measured in the multijet data sample and the two correction factors is used to estimate the mistag rate for light-flavor jets $\epsilon_{l}\left(p_{T}, \eta\right)$ :

$$
\epsilon_{l}\left(p_{T}, \eta\right)=\epsilon_{n e g}^{\text {data }}\left(p_{T}, \eta\right) \cdot S F_{h f}\left(p_{T}, \eta\right) \cdot S F_{l l}\left(p_{T}, \eta\right)
$$



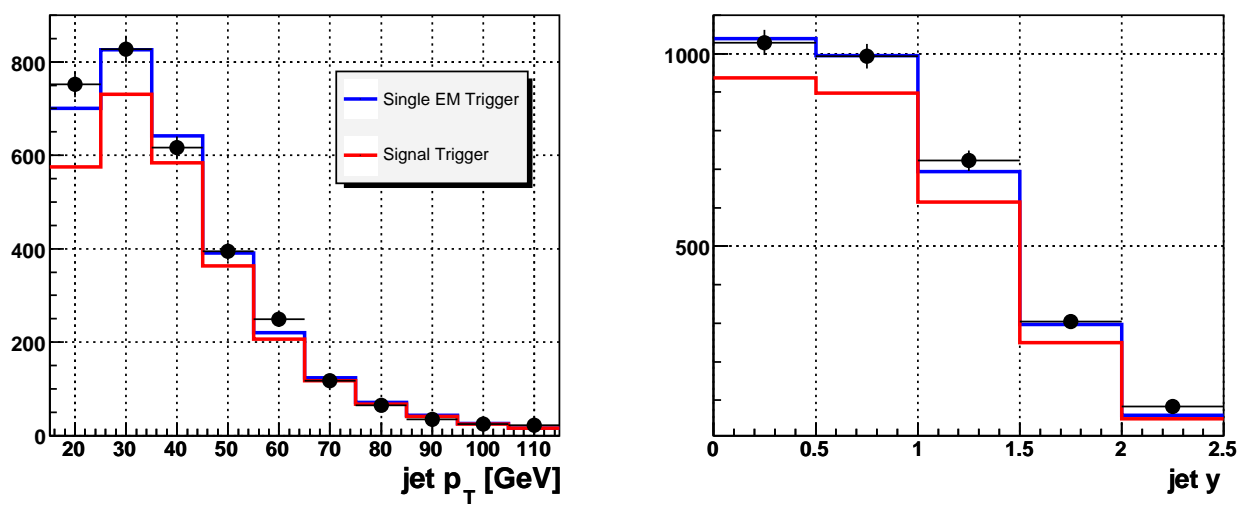

Figure 7.15: The $p_{T}$ (left) and $y$ (right) distributions of negatively tagged jets in the multijet sample compared with the distributions predicted by the negative tag rate parameterizations.
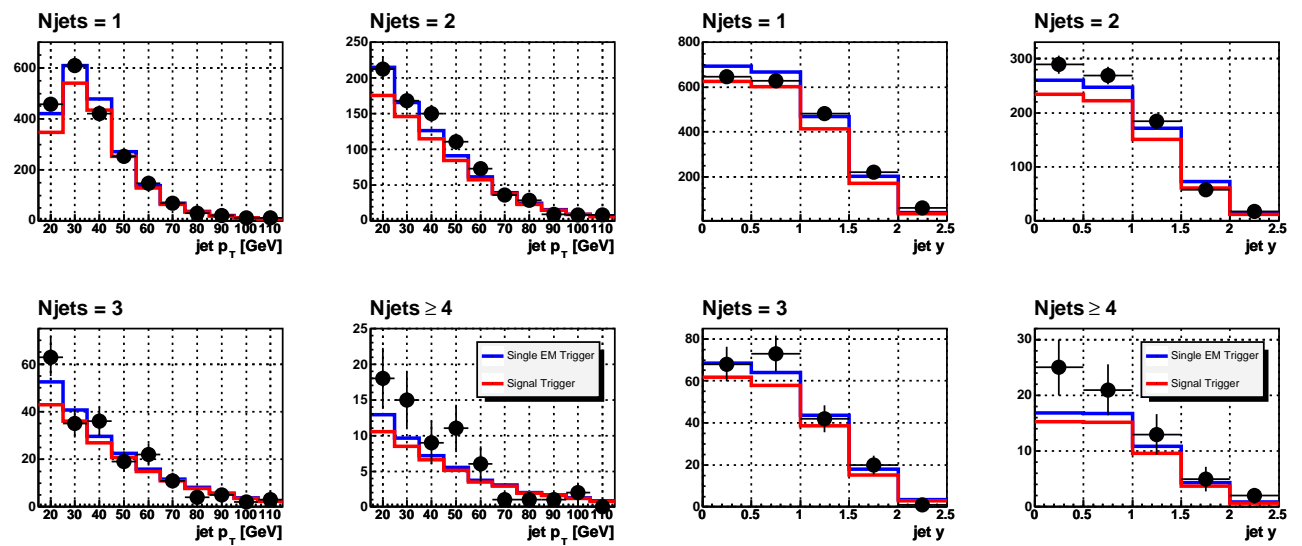

Figure 7.16: The $p_{T}$ (left four plots) and $y$ (right four plots) distributions of negatively tagged jets in the multijet sample split in jet multiplicity bins. The histograms correspond to the distributions predicted by the negative tag rate parameterizations. 


\begin{tabular}{lcccc}
\hline \hline & 1 jet & 2 jet & 3 jet & $\geq 4$ jet \\
\hline \hline \multicolumn{5}{c}{$\mu+$ jets channel } \\
\hline \hline$N_{E M}^{\text {pred }}$ & $23.9 \pm 4.9$ & $15.1 \pm 3.9$ & $4.80 \pm 2.19$ & $1.77 \pm 1.33$ \\
$N_{\text {signal }}^{\text {pred }}$ & $22.3 \pm 4.7$ & $13.9 \pm 3.7$ & $4.40 \pm 2.10$ & $1.63 \pm 1.28$ \\
\hline$N_{\text {neg tag }}^{\text {obs }}$ & 18 & 14 & 4 & 3 \\
\hline \hline \multicolumn{5}{c}{$e+$ jets channel } \\
\hline \hline$N_{E M}^{\text {pred }}$ & $24.5 \pm 4.9$ & $15.4 \pm 3.9$ & $5.5 \pm 2.3$ & $2.39 \pm 1.54$ \\
$N_{\text {signal }}^{\text {pred }}$ & $22.6 \pm 4.8$ & $14.1 \pm 3.8$ & $5.0 \pm 2.2$ & $2.19 \pm 1.48$ \\
\hline$N_{\text {neg tag }}^{\text {obs }}$ & 25 & 14 & 4 & 5 \\
\hline \hline \multicolumn{5}{c}{$\ell+$ jets channel } \\
\hline \hline$N_{E M}^{\text {pred }}$ & $48.4 \pm 7.0$ & $30.5 \pm 5.5$ & $10.3 \pm 3.2$ & $4.16 \pm 2.04$ \\
$N_{\text {signal }}^{\text {pred }}$ & $44.9 \pm 6.7$ & $28.0 \pm 5.3$ & $9.4 \pm 3.1$ & $3.82 \pm 1.96$ \\
\hline$N_{\text {neg tag }}^{\text {obs }}$ & 43 & 28 & 8 \\
\hline \hline \multicolumn{5}{c}{}
\end{tabular}

Table 7.1: Number of observed and predicted negative tags in the preselected signal samples. $N_{E M}^{\text {pred }}$ is the prediction obtained with the standard parametrization from events passing single EM triggers and $N_{\text {signal }}^{\text {pred }}$ is the prediction from the alternative parametrization obtained from events passing the $e+$ jets signal trigger.
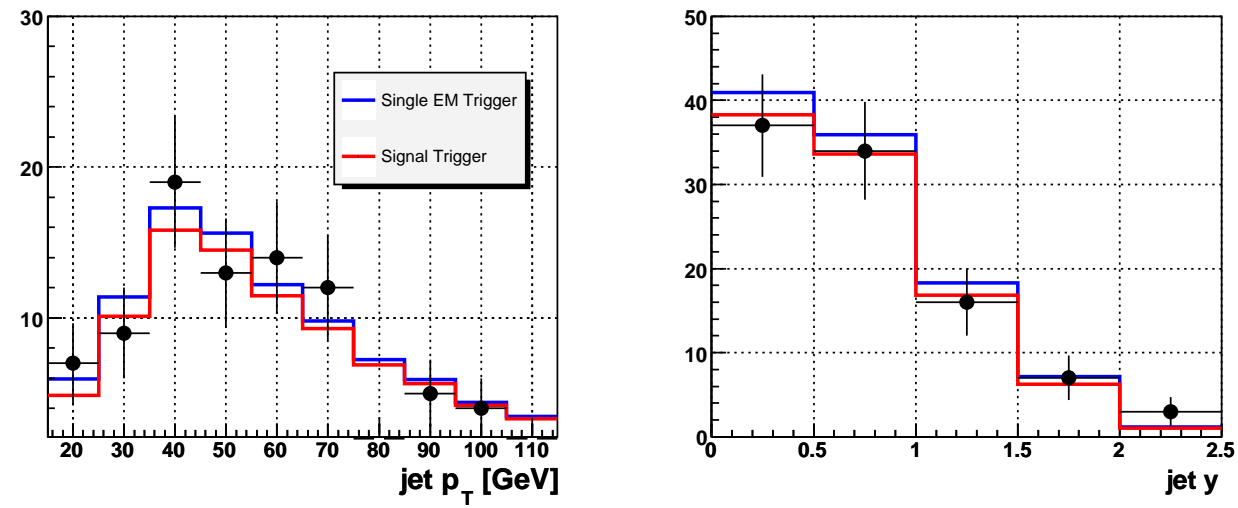

Figure 7.17: The $p_{T}$ (left) and $y$ (right) distributions of negatively tagged jets in the preselected $\ell+$ jets signal sample compared with the distributions predicted by the negative tag rate parameterizations. 

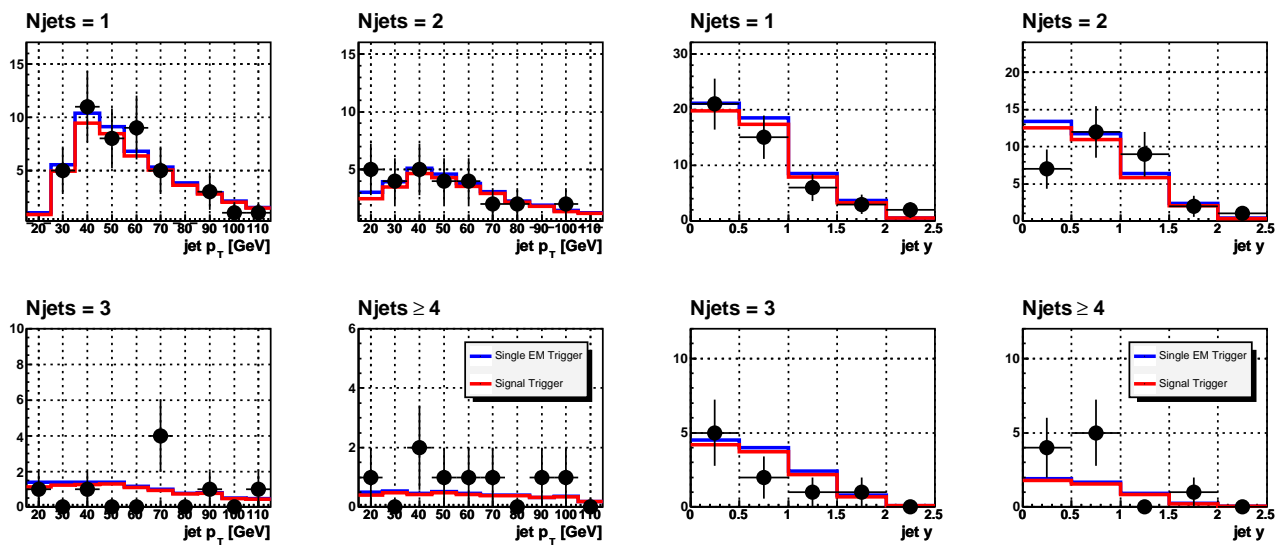

Figure 7.18: The $p_{T}$ (left four plots) and $y$ (right four plots) distributions of negatively tagged jets in the preselected $\ell+$ jets signal sample split in jet multiplicity bins. The histograms correspond to the distributions predicted by the negative tag rate parameterizations.
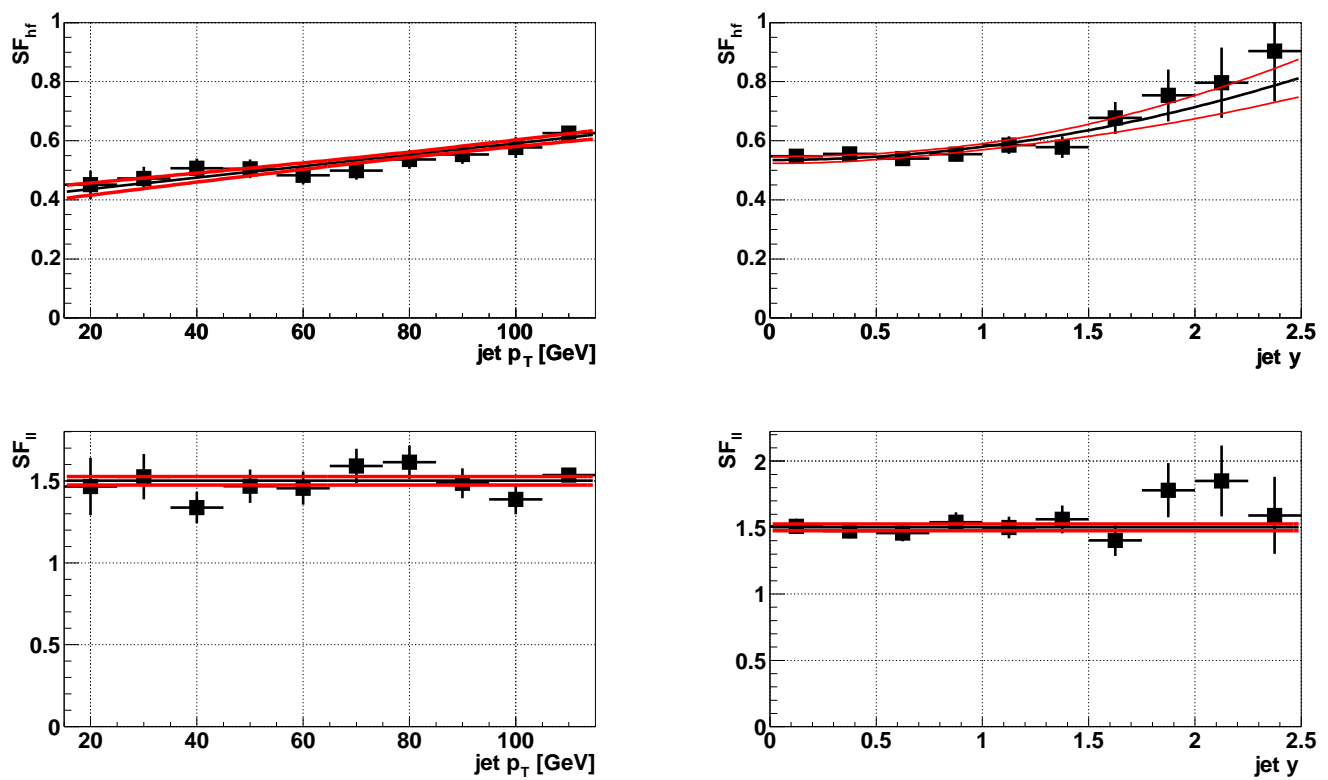

Figure 7.19: Correction factors for the contribution of heavy-flavor jets to the measured negative tag rate, $S F_{h f}$ (upper plots), and the contribution to the mistag rate from long-lived particles, $S F_{l l}$ (lower plots). The left two plots show the jet $p_{T}$ dependence and the right two plots show the jet $y$ dependence. 


\section{Chapter 8}

\section{The $b$-tagged Signal Sample}

The preselection criteria described in Chapter 6 reduces the total $\mathrm{D} \varnothing$ data sample to a couple of thousand events. The preselected sample is dominated by background events. To reduce the background, events are required to have at least one $b$-tagged jet. As was shown in Chapter 7 , the efficiency to tag a $b$-jet with the SVT algorithm is approximately $35 \%$, while the mistag rate for light-flavor jets is less than $0.5 \%$. This leads to a very good discrimination against events containing only light-flavor jets while retaining the majority of the $t \bar{t}$ events.

To increase the statistical sensitivity in the $t \bar{t}$ cross section measurement, single tagged and double tagged events are considered separately. The double tagged events have a very high purity, but the statistics is lower than for single tagged events.

Counting the single and double tagged events separately also allows for a measurement of the ratio $R=B(t \rightarrow W b) / B(t \rightarrow W q)$. The number of expected $t \bar{t}$ events with 0,1 and $2 b$-tagged jets depend on the $t \bar{t}$ cross section, the $b$-tagging efficiency and the ratio $R$. This is described further in Chapter 9.

\subsection{Event Tagging Probability}

For all processes, except instrumental multijet events, the expected number of events with $n$ tagged jets, $N_{i}^{t a g}$ ( $n$ tags), in the final sample for process $i$ is

$$
N_{i}^{\text {tag }}(n \text { tags })=P_{i}^{\text {tag }}(n \text { tags }) \cdot N_{i}^{\text {presel }} \quad(n=0,1,2)
$$

where $N_{i}^{\text {presel }}$ is the number of preselected events and $P_{i}^{\text {tag }}(n$ tags $)$ is the average probability for an event to have $n$ tagged jets. Multijet events are treated separately, as is described in Sec. 8.1.1.

The event tagging probability depends on the number of jets in the event and the tagging probability for each jet. The tagging probability per jet, $P_{j e t(\alpha)}\left(p_{T}, y\right)$, is given by Eq. 7.1 and depends on the jet flavor $\alpha$, jet $p_{T}$ and jet $y$. The average event tagging probabilities are obtained from Monte Carlo simulation. The probability for 
an event to have at least one $b$-tagged jet is given by

$$
P_{\text {event }}^{\text {tag }}(\geq 1 \text { tags })=1-\prod_{i=1}^{N_{j e t s}}\left(1-P_{j e t(\alpha)}^{i}\left(p_{T}, y\right)\right)
$$

where $N_{\text {jets }}$ is the number of jets with $p_{T}>15 \mathrm{GeV} / c$ and $|y|<2.5$. Given Eq. 8.2, the event tagging probabilities for 0,1 and $2 b$-tagged jets can be calculated as:

$$
\begin{aligned}
P_{\text {event }}^{\text {tag }}(0 \text { tags }) & =\prod_{i=1}^{N_{\text {jets }}}\left(1-P_{\text {jet }(\alpha)}^{i}\left(p_{T}, y\right)\right) \\
P_{\text {event }}^{\text {tag }}(1 \mathrm{tag}) & =\sum_{j=1}^{N_{\text {jets }}} P_{\text {jet }(\alpha)}^{j}\left(p_{T}, y\right) \prod_{i=1 ; i \neq j}^{N_{\text {jets }}}\left(1-P_{j e t(\alpha)}^{i}\left(p_{T}, y\right)\right) \\
P_{\text {event }}^{\text {tag }}(\geq 2 \text { tags }) & =P_{\text {event }}^{\text {tag }}(\geq 1 \text { tags })-P_{\text {event }}^{\text {tag }}(1 \text { tag })
\end{aligned}
$$

The average event tagging probability is obtained by averaging over all simulated events passing the preselection criteria.

\subsubsection{The Multijet Background}

The instrumental multijet contribution to the tagged sample is fully determined from data. The Matrix Method, described in Sec. 6.3, is applied directly to the tagged events in the loose and the tight preselected samples to get the estimated number of tagged multijet events:

$$
N_{\text {instr }}^{\text {tag }}=\varepsilon_{\text {instr }} \cdot \frac{\varepsilon_{\text {lept }} N_{\text {loose }}^{\text {tag }}-N_{\text {tight }}^{\text {tag }}}{\varepsilon_{\text {lept }}-\varepsilon_{\text {instr }}} .
$$

The probability $\varepsilon_{l e p t}$ for an event with a true isolated lepton to pass the final lepton selection is expected to be independent of the flavor composition of the jets in the event. The measurement of $\varepsilon_{\text {lept }}$ performed in the untagged sample, shown in Tab. 6.4, is therefore used also in the tagged sample.

The probability for a misreconstructed event to pass the lepton selection, $\varepsilon_{\text {instr }}$, could depend on the jet flavor in the event. Therefore $\varepsilon_{\text {instr }}$ is re-derived using only $b$-tagged events in the low $\mathscr{E}_{T}$ samples described in Sec. 6.3. The $\varepsilon_{\text {instr }}$ values derived in the tagged and the untagged samples are found to be the same within the uncertainties, as shown in Tab. 8.1. Since no dependence of $\varepsilon_{\text {instr }}$ on the jet flavor is observed, the $\varepsilon_{i n s t r}$ values derived in the untagged sample are used also in the tagged sample. The observed number of events in the loose and the tight samples are shown in Tab. 8.2 for single tagged events and in Tab. 8.3 for double tagged events. Also shown is the number of multijet background events predicted by the Matrix Method. 


\begin{tabular}{lcc}
\hline \hline$\varepsilon_{\text {instr }}$ & Untagged events & $b$-tagged events \\
\hline$e+$ jets channel & $15 \pm 3$ & $12 \pm 3$ \\
$\mu+$ jets channel & $8.5 \pm 3$ & $7 \pm 3$ \\
\hline \hline
\end{tabular}

Table 8.1: Comparison of $\varepsilon_{\text {instr }}$ (in \%) measured in untagged and tagged events in the low $\not_{T}$ sample.

\begin{tabular}{l|cccc}
\hline \hline & 1 jet & 2 jets & 3 jets & $\geq 4$ jets \\
\hline \hline \multicolumn{5}{c}{$e+$ jets channel } \\
\hline \hline$N_{\text {loose }}^{\text {tag }}$ & 122 & 152 & 74 & 63 \\
$N_{\text {tight }}^{\text {tag }}$ & 69 & 83 & 36 & 38 \\
\hline$N_{\text {instr }}^{\text {tag }}$ & $7.1 \pm 1.6$ & $9.0 \pm 1.9$ & $5.8 \pm 1.4$ & $3.3 \pm 1.2$ \\
\hline \hline \multicolumn{5}{c}{$\mu+$ jets channel } \\
\hline \hline$N_{\text {loose }}^{\text {tag }}$ & 161 & 149 & 76 & 38 \\
$N_{\text {tight }}^{\text {tag }}$ & 68 & 62 & 43 & 24 \\
\hline$N_{\text {instr }}^{\text {tag }}$ & $7.8 \pm 0.9$ & $7.3 \pm 0.9$ & $2.5 \pm 0.6$ & $0.8 \pm 0.4$ \\
\hline \hline
\end{tabular}

Table 8.2: Number of observed single tagged events in the loose and tight preselected samples, together with the expected number of single tagged multijet events predicted by the Matrix Method.

\subsubsection{W+jets Background}

The event tagging probabilities for $W+$ jets events are determined separately for each flavor configuration described in Sec. 5.2.2. These event tagging probabilities $P_{\Phi}^{t a g}$ are then weighted according to their flavor fractions $F_{\Phi}$, shown in Tab. 5.4, to obtain the event tagging probability $P_{W+\text { jets }}^{t a g}$ for $W+$ jets events:

$$
P_{W+\mathrm{jets}}^{t a g}=\sum_{\Phi} F_{\Phi} P_{\Phi}^{t a g}
$$

Table 8.4 shows the event tagging probabilities $P_{\Phi}^{t a g}$ for single tagged events. For each jet multiplicity bin, the event tagging probabilities $P_{\Phi}^{t a g}$ are weighted according to Eq. 8.7. The resulting event tagging probability $P_{W+\text { jets }}^{t a g}$ is shown in the last line in Tab. 8.4. The corresponding event tagging probabilities for double tagged events are shown in Tab. 8.5.

An alternative way of obtaining the event tagging probability for $W+$ light jets events is to apply the mistag rate, defined in Eq. 7.14, directly to the events in the preselected signal sample. Since the preselected sample is dominated by $W+$ light jets events, this method has the advantage of taking the kinematic information 


\begin{tabular}{l|ccc}
\hline \hline & 2 jets & 3 jets & $\geq 4$ jets \\
\hline \hline \multicolumn{4}{|c}{$e+$ jets channel } \\
\hline \hline$N_{\text {loose }}^{\text {tag }}$ & 7 & 8 & 12 \\
$N_{\text {tight }}^{\text {tag }}$ & 6 & 6 & 9 \\
\hline$N_{\text {instr }}^{\text {tag }}$ & $<0.1$ & $<0.1$ & $<0.1$ \\
\hline \hline \multicolumn{4}{|c}{$\mu+$ jets channel } \\
\hline \hline$N_{\text {loose }}^{\text {tag }}$ & 9 & 3 & 5 \\
$N_{\text {tight }}^{\text {tag }}$ & 9 & 3 & 5 \\
\hline$N_{\text {instr }}^{\text {tag }}$ & $<0.1$ & $<0.1$ & $<0.1$ \\
\hline \hline
\end{tabular}

Table 8.3: Number of observed double tagged events in the loose and tight preselected samples, together with the expected number of double tagged multijet events predicted by the Matrix Method.

directly from data. The obtained event tagging probabilities are shown in Tab. 8.6, and are in good agreement with those in Tab. 8.4 derived from simulated events.

\subsubsection{Other Physics Backgrounds}

There are physics backgrounds other than $W+$ jets, as discussed in Sec. 5.2.3. They include production of $W W, W Z, Z Z, Z \rightarrow \tau \tau$ and single top quarks.

The average event tagging probabilities for events from single top quark production in the $s$ - and $t$-channels, diboson production and $Z \rightarrow \tau^{+} \tau^{-}$are listed in Tab. 8.7 for single tagged events and in Tab. 8.8 for double tagged events.

\subsubsection{The $t \bar{t}$ Signal}

The event tagging probabilities for $t \bar{t} \rightarrow \ell+$ jets and $t \bar{t} \rightarrow \ell \ell$ are listed in Tab. 8.9 for single tagged events and in Tab. 8.10 for double tagged events.

\subsection{Final Sample Composition}

The observed and predicted number of single tagged events are summarized in Tab. 8.11 for the $e+$ jets and $\mu+$ jets channels and in Tab. 8.12 for the combined $\ell+$ jets channel. The corresponding results for double tagged events are shown in Tab. 8.13 for the $e+$ jets and $\mu+$ jets channels and in Tab. 8.14 for the combined $\ell+$ jets channel.

In Figs. 8.1, 8.2 and 8.3 the observed number of tagged events in data is compared to the total signal and background predictions assuming a $t \bar{t}$ production cross section of $7 \mathrm{pb}$. The largest background in the first jet multiplicity bin comes from 


\begin{tabular}{|c|c|c|c|c|}
\hline & $\overline{W+1 \text { jet }}$ & $\bar{~} W+2$ jets & $\overline{W+3 \text { jets }}$ & $\bar{W}+\geq 4$ jets \\
\hline & \multicolumn{4}{|c|}{ "e+jets channel } \\
\hline$\overline{W+\text { light }}$ & $0.26 \pm 0.01$ & $0.45 \pm 0.02$ & $\overline{c 0.78 \pm 0.04}$ & $1.01 \pm 0.02$ \\
\hline$W(c \bar{c})$ & $7.4 \pm 0.1$ & $7.0 \pm 0.1$ & $7.4 \pm 0.1$ & $7.9 \pm 0.5$ \\
\hline$W(b \bar{b})$ & $32.9 \pm 0.2$ & $31.3 \pm 0.3$ & $31.1 \pm 0.2$ & $31.0 \pm 1.0$ \\
\hline$W c$ & $7.8 \pm 0.1$ & $7.8 \pm 0.1$ & $8.1 \pm 0.2$ & $8.4 \pm 0.5$ \\
\hline$W c \bar{c}$ & & $12.9 \pm 0.2$ & $12.9 \pm 0.5$ & $14.1 \pm 0.3$ \\
\hline$W b \bar{b}$ & & $42.9 \pm 0.3$ & $42.1 \pm 0.5$ & $41.8 \pm 0.3$ \\
\hline \multirow[t]{2}{*}{$W+$ jets } & $0.93 \pm 0.01$ & $2.10 \pm 0.02$ & $3.13 \pm 0.04$ & $4.25 \pm 0.05$ \\
\hline & \multicolumn{4}{|c|}{ " $\mu+$ jets channel } \\
\hline$\overline{W+\text { light }}$ & $0.34 \pm 0.01$ & $\overline{0.56 \pm 0.02}$ & $\overline{00.78 \pm 0.04}$ & $1.04 \pm 0.02$ \\
\hline$W(c \bar{c})$ & $8.4 \pm 0.1$ & $7.6 \pm 0.2$ & $7.6 \pm 0.1$ & $8.5 \pm 0.4$ \\
\hline$W(b \bar{b})$ & $35.7 \pm 0.2$ & $32.2 \pm 0.3$ & $31.1 \pm 0.2$ & $31.4 \pm 0.8$ \\
\hline$W c$ & $8.5 \pm 0.1$ & $8.4 \pm 0.1$ & $8.7 \pm 0.2$ & $9.0 \pm 0.4$ \\
\hline$W c \bar{c}$ & & $14.3 \pm 0.2$ & $13.6 \pm 0.4$ & $13.8 \pm 0.3$ \\
\hline$W b \bar{b}$ & & $43.7 \pm 0.3$ & $42.8 \pm 0.4$ & $41.9 \pm 0.3$ \\
\hline$\overline{W+\text { jets }}$ & $1.06 \pm 0.01$ & $2.29 \pm 0.02$ & $3.22 \pm 0.04$ & $4.34 \pm 0.04$ \\
\hline
\end{tabular}

Table 8.4: Single tag probabilities (in \%) for preselected $W+$ jets events. Only uncertainties from limited statistics are shown.

$W+$ light jet and $W c$ production. The contribution from $W$ boson production in association with $b$-jets dominates starting from the second jet multiplicity bin. The $t \bar{t}$ signal is expected to contribute primarily to the third and fourth jet multiplicity bins. The agreement between the observed and predicted number of events in the first two jet multiplicity bins is a strong confirmation of the validity of the background predictions. 


\begin{tabular}{|c|c|c|c|}
\hline & $\overline{W+2 \text { jets }}$ & $\overline{W+3 \text { jets }}$ & $\overline{W+\geq 4 \text { jets }}$ \\
\hline & \multicolumn{3}{|c|}{$e+$ jets channel } \\
\hline$\overline{W+\text { light }}$ & $<0.01$ & $<<0.01$ & $<0.01$ \\
\hline$W(c \bar{c})$ & $0.026 \pm 0.003$ & $0.053 \pm 0.003$ & $0.07 \pm 0.01$ \\
\hline$W(b \bar{b})$ & $0.43 \pm 0.06$ & $0.70 \pm 0.04$ & $0.64 \pm 0.12$ \\
\hline$W c$ & $0.015 \pm 0.001$ & $0.032 \pm 0.002$ & $0.06 \pm 0.01$ \\
\hline$W c \bar{c}$ & $0.51 \pm 0.02$ & $0.54 \pm 0.05$ & $0.71 \pm 0.04$ \\
\hline$W b \bar{b}$ & $9.7 \pm 0.2$ & $9.6 \pm 0.4$ & $9.8 \pm 0.3$ \\
\hline$W+$ jets & $0.122 \pm 0.003$ & $0.21 \pm 0.01$ & $0.34 \pm 0.01$ \\
\hline & \multicolumn{3}{|c|}{ 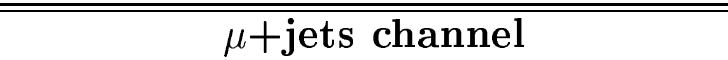 } \\
\hline$\overline{W+\text { light }}$ & $\overline{<<0.01}$ & $<00.01$ & $<<0.01$ \\
\hline$W(c \bar{c})$ & $0.029 \pm 0.003$ & $0.059 \pm 0.003$ & $0.09 \pm 0.02$ \\
\hline$W(b \bar{b})$ & $0.61 \pm 0.08$ & $0.63 \pm 0.04$ & $1.11 \pm 0.22$ \\
\hline$W c$ & $0.019 \pm 0.001$ & $0.037 \pm 0.002$ & $0.06 \pm 0.01$ \\
\hline$W c \bar{c}$ & $0.61 \pm 0.02$ & $0.57 \pm 0.04$ & $0.67 \pm 0.03$ \\
\hline$W b \bar{b}$ & $10.7 \pm 0.2$ & $10.3 \pm 0.4$ & $9.9 \pm 0.2$ \\
\hline$W+$ jets & $0.137 \pm 0.003$ & $0.23 \pm 0.01$ & $0.35 \pm 0.01$ \\
\hline
\end{tabular}

Table 8.5: Double tag probabilities (in \%) for preselected $W+$ jets events. Only uncertainties from limited statistics are shown.

\begin{tabular}{|c|c|c|c|c|}
\hline & 1 jet & 2 jets & 3 jets & $\geq 4$ jets \\
\hline & \multicolumn{4}{|c|}{$e+$ jets channel } \\
\hline$\overline{W+\text { light }}$ & $0.264 \pm 0.004$ & $0.46 \pm 0.01$ & $0.66 \pm 0.03$ & $1.02 \pm 0.08$ \\
\hline & \multicolumn{4}{|c|}{$\mu \mu+$ jets channel } \\
\hline$\overline{W+\text { light }}$ & 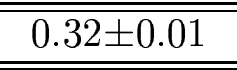 & $\overline{0.52 \pm 0.01}$ & $\overline{00.69 \pm 0.03}$ & $\bar{~} 1.03 \pm 0.10$ \\
\hline
\end{tabular}

Table 8.6: Single tag probabilities (in \%) obtained from applying the mistag rate to the preselected signal sample. 


\begin{tabular}{|c|c|c|c|c|}
\hline & 1 jet & 2 jets & 3 jets & $\geq 4$ jets \\
\hline & \multicolumn{4}{|c|}{$e+$ jets channel } \\
\hline $\bar{t} t b \rightarrow \ell \nu b b$ & $37.5 \pm 0.2$ & 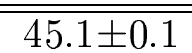 & $=45.3 \pm 0.1$ & $45.4 \pm 0.3$ \\
\hline$t b q \rightarrow \ell \nu b b j$ & $29.9 \pm 0.4$ & $36.4 \pm 0.2$ & $39.7 \pm 0.2$ & $41.7 \pm 0.3$ \\
\hline$W W \rightarrow \ell \nu j j$ & $2.54 \pm 0.15$ & $4.10 \pm 0.12$ & $4.30 \pm 0.32$ & $5.1 \pm 1.3$ \\
\hline$W Z \rightarrow \ell \nu j j$ & $12.6 \pm 0.6$ & $13.5 \pm 0.5$ & $13.6 \pm 1.3$ & $10.8 \pm 4.2$ \\
\hline$W Z \rightarrow j j \ell \ell$ & $2.67 \pm 0.36$ & $4.34 \pm 0.32$ & $4.71 \pm 0.47$ & $4.71 \pm 1.56$ \\
\hline$Z Z \rightarrow \ell \ell j j$ & $12.8 \pm 1.3$ & $17.0 \pm 1.2$ & $16.0 \pm 1.8$ & $8.5 \pm 3.4$ \\
\hline$Z / \gamma^{*} \rightarrow \tau \tau$ & $0.78 \pm 0.20$ & $2.69 \pm 0.74$ & $1.36 \pm 0.34$ & $9.1 \pm 5.1$ \\
\hline \multicolumn{5}{|c|}{ " $\mu+$ jets channel } \\
\hline$\overline{t b \rightarrow \ell \nu b b}$ & $37.5 \pm 0.2$ & $\overline{445.1 \pm 0.1}$ & $4 \quad 45.1 \pm 0.1$ & 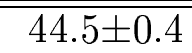 \\
\hline$t b q \rightarrow \ell \nu b b j$ & $29.9 \pm 0.4$ & $35.9 \pm 0.2$ & $39.5 \pm 0.2$ & $40.8 \pm 0.4$ \\
\hline$W W \rightarrow \ell \nu j j$ & $2.96 \pm 0.18$ & $4.09 \pm 0.11$ & $4.25 \pm 0.31$ & $5.0 \pm 1.1$ \\
\hline$W Z \rightarrow \ell \nu j j$ & $11.4 \pm 0.7$ & $11.2 \pm 0.5$ & $9.7 \pm 1.2$ & $16.8 \pm 3.8$ \\
\hline$W Z \rightarrow j j \ell \ell$ & $2.46 \pm 0.21$ & $3.65 \pm 0.15$ & $4.27 \pm 0.42$ & $3.47 \pm 0.93$ \\
\hline$Z Z \rightarrow \ell \ell j j$ & $12.2 \pm 1.1$ & $12.3 \pm 0.7$ & $13.6 \pm 1.5$ & $15.7 \pm 5.3$ \\
\hline$Z / \gamma^{*} \rightarrow \tau \tau$ & $0.65 \pm 0.16$ & $1.36 \pm 0.30$ & $1.80 \pm 0.88$ & $3.38 \pm 1.46$ \\
\hline
\end{tabular}

Table 8.7: Single tag probabilities (in \%) after full preselection for physics backgrounds other than $W+$ jets. Only uncertainties from limited statistics are shown. 


\begin{tabular}{l|ccc}
\hline \hline \multicolumn{4}{c}{$e+$ jets channel } \\
\hline \hline$t b \rightarrow \ell \nu b b$ & $11.8 \pm 0.1$ & $12.4 \pm 0.2$ & $12.6 \pm 0.4$ \\
$t b q \rightarrow \ell \nu b b j$ & $1.44 \pm 0.06$ & $5.8 \pm 0.1$ & $8.4 \pm 0.2$ \\
$W W \rightarrow \ell \nu j j$ & $0.011 \pm 0.001$ & $0.015 \pm 0.002$ & $0.05 \pm 0.03$ \\
$W Z \rightarrow \ell \nu j j$ & $2.78 \pm 0.14$ & $2.85 \pm 0.36$ & $2.69 \pm 1.20$ \\
$W Z \rightarrow j j \ell \ell$ & $0.013 \pm 0.002$ & $0.027 \pm 0.005$ & $0.08 \pm 0.06$ \\
$Z Z \rightarrow \ell \ell j j$ & $3.21 \pm 0.34$ & $2.82 \pm 0.47$ & $1.95 \pm 0.97$ \\
$Z / \gamma^{*} \rightarrow \tau \tau$ & $0.06 \pm 0.05$ & $<0.01$ & $0.90 \pm 0.69$ \\
\hline \hline \multicolumn{4}{c}{$\mu+$ jets channel } \\
\hline \hline$t b \rightarrow \ell \nu b b$ & $11.6 \pm 0.1$ & $12.1 \pm 0.2$ & $11.9 \pm 0.4$ \\
$t b q \rightarrow \ell \nu b b j$ & $1.38 \pm 0.06$ & $5.6 \pm 0.1$ & $8.0 \pm 0.3$ \\
$W W \rightarrow \ell \nu j j$ & $0.010 \pm 0.001$ & $0.023 \pm 0.005$ & $0.06 \pm 0.02$ \\
$W Z \rightarrow \ell \nu j j$ & $2.28 \pm 0.12$ & $1.81 \pm 0.28$ & $3.20 \pm 1.00$ \\
$W Z \rightarrow j j \ell \ell$ & $<0.01$ & $0.04 \pm 0.02$ & $0.03 \pm 0.01$ \\
$Z Z \rightarrow \ell \ell j j$ & $2.30 \pm 0.18$ & $2.85 \pm 0.42$ & $3.34 \pm 1.35$ \\
$Z / \gamma^{*} \rightarrow \tau \tau$ & $0.05 \pm 0.04$ & $0.12 \pm 0.12$ & $0.010 \pm 0.004$ \\
\hline \hline
\end{tabular}

Table 8.8: Double tag probabilities (in \%) after full preselection for physics backgrounds other than $W+$ jets. Only uncertainties from limited statistics are shown.

\begin{tabular}{|c|c|c|c|c|}
\hline & 1 jet & 2 jets & 3 jets & $\geq 4$ jets \\
\hline & \multicolumn{4}{|c|}{$e+$ jets channel } \\
\hline$t \bar{t} \rightarrow \ell+$ jets & $24.6 \pm 1.8$ & $39.2 \pm 0.4$ & 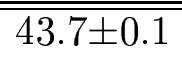 & $\overline{45.5 \pm 0.1}$ \\
\hline$t \bar{t} \rightarrow \ell \ell$ & $38.8 \pm 0.3$ & $45.1 \pm 0.1$ & $45.7 \pm 0.1$ & $46.0 \pm 0.2$ \\
\hline \multicolumn{5}{|c|}{$\mu$ +jets channel } \\
\hline$\overline{t \bar{t} \rightarrow \ell+\text { jets }}$ & $25.5 \pm 2.2$ & $37.9 \pm 0.4$ & 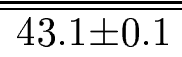 & 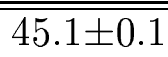 \\
\hline$t \bar{t} \rightarrow \ell \ell$ & $38.6 \pm 0.3$ & $45.0 \pm 0.1$ & $45.4 \pm 0.1$ & $45.5 \pm 0.2$ \\
\hline
\end{tabular}

Table 8.9: Single tag probabilities (in \%) for $t \bar{t}$ events after preselection. Only uncertainties from limited statistics are shown. 


\begin{tabular}{|c|c|c|c|}
\hline & 2 jets & 3 jets & $\geq 4$ jets \\
\hline & \multicolumn{3}{|c|}{$e+$ jets channel } \\
\hline$t \bar{t} \rightarrow \ell+$ jets & $4.9 \pm 0.2$ & $10.6 \pm 0.1$ & $14.5 \pm 0.1$ \\
\hline$t \bar{t} \rightarrow \ell \ell$ & $11.3 \pm 0.1$ & $12.4 \pm 0.1$ & $13.5 \pm 0.3$ \\
\hline & \multicolumn{3}{|c|}{$\mu+$ jets channel } \\
\hline$t \bar{t} \rightarrow \ell+$ jets & $4.3 \pm 0.2$ & $10.3 \pm 0.1$ & $13.9 \pm 0.1$ \\
\hline$t \bar{t} \rightarrow \ell \ell$ & $11.2 \pm 0.1$ & $12.1 \pm 0.1$ & $12.7 \pm 0.3$ \\
\hline
\end{tabular}

Table 8.10: Double tag probabilities (in \%) for $t \bar{t}$ events after preselection. Only uncertainties from limited statistics are shown. 


\begin{tabular}{|c|c|c|c|c|}
\hline & 1 jet & 2 jets & 3 jets & $\geq 4$ jets \\
\hline & \multicolumn{4}{|c|}{ e+jets channel } \\
\hline$\overline{\bar{W}+\text { light }}$ & $17.9 \pm 0.5$ & $9.9 \pm 0.5$ & $3.64 \pm 0.28$ & $0.94 \pm 0.13$ \\
\hline$W(c \bar{c})$ & $6.3 \pm 0.1$ & $3.61 \pm 0.13$ & $1.27 \pm 0.07$ & $0.42 \pm 0.06$ \\
\hline$W(b \bar{b})$ & $17.9 \pm 0.3$ & $10.3 \pm 0.3$ & $3.28 \pm 0.19$ & $1.03 \pm 0.15$ \\
\hline$W c$ & $25.2 \pm 0.5$ & $12.1 \pm 0.4$ & $2.79 \pm 0.17$ & $0.41 \pm 0.06$ \\
\hline$W c \bar{c}$ & & $4.88 \pm 0.16$ & $2.02 \pm 0.14$ & $0.75 \pm 0.11$ \\
\hline$W b \bar{b}$ & & $11.7 \pm 0.3$ & $4.35 \pm 0.25$ & $1.37 \pm 0.19$ \\
\hline$W+$ jets & $67.4 \pm 0.8$ & $52.5 \pm 0.8$ & $17.4 \pm 0.5$ & $4.92 \pm 0.31$ \\
\hline Multijet & $7.1 \pm 1.6$ & $9.0 \pm 1.9$ & $5.8 \pm 1.4$ & $3.27 \pm 1.15$ \\
\hline Single top & $1.45 \pm 0.02$ & $4.27 \pm 0.04$ & $1.77 \pm 0.03$ & $0.53 \pm 0.02$ \\
\hline$t \bar{t} \rightarrow \ell \ell$ & $1.02 \pm 0.03$ & $3.84 \pm 0.06$ & $2.04 \pm 0.04$ & $0.56 \pm 0.02$ \\
\hline Diboson \& $Z \rightarrow \tau \tau$ & $1.08 \pm 0.05$ & $2.59 \pm 0.08$ & $0.40 \pm 0.03$ & $0.06 \pm 0.03$ \\
\hline Total bkg. & $78.1 \pm 1.8$ & $72.1 \pm 2.0$ & $27.4 \pm 1.5$ & $9.3 \pm 1.2$ \\
\hline Syst. Unc. & $+7.14-8.82$ & $+7.80-8.42$ & $+2.75-2.91$ & $+0.89-0.90$ \\
\hline$t \bar{t} \rightarrow \ell+$ jets & $0.23 \pm 0.03$ & $3.90 \pm 0.11$ & $12.8 \pm 0.2$ & $17.5 \pm 0.2$ \\
\hline Total Expect & $78.3 \pm 1.8$ & $76.0 \pm 2.0$ & $40.1 \pm 1.5$ & $26.9 \pm 1.2$ \\
\hline Syst. Unc. & $+7.15-8.84$ & $+7.88-8.53$ & $+2.89-3.02$ & $+2.26-2.47$ \\
\hline \multirow[t]{2}{*}{ Observed } & 69 & 83 & 36 & 38 \\
\hline & \multicolumn{4}{|c|}{ 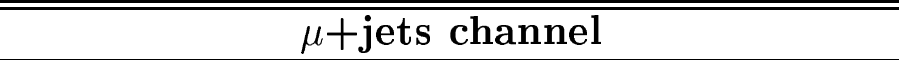 } \\
\hline $\bar{~} W+$ light & $18.9 \pm 0.5$ & 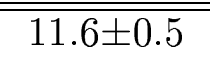 & $3.52 \pm 0.25$ & $0.86 \pm 0.11$ \\
\hline$W(c \bar{c})$ & $5.9 \pm 0.1$ & $3.64 \pm 0.11$ & $1.25 \pm 0.06$ & $0.39 \pm 0.05$ \\
\hline$W(b \bar{b})$ & $16.0 \pm 0.2$ & $10.0 \pm 0.2$ & $3.16 \pm 0.15$ & $0.92 \pm 0.12$ \\
\hline$W c$ & $22.6 \pm 0.4$ & $12.1 \pm 0.3$ & $2.90 \pm 0.15$ & $0.38 \pm 0.05$ \\
\hline$W c \bar{c}$ & & $5.1 \pm 0.1$ & $2.06 \pm 0.12$ & $0.64 \pm 0.08$ \\
\hline$W b \bar{b}$ & & $11.2 \pm 0.3$ & $4.27 \pm 0.21$ & $1.21 \pm 0.15$ \\
\hline$W+$ jets & $63.5 \pm 0.7$ & $53.5 \pm 0.7$ & $17.2 \pm 0.4$ & $4.40 \pm 0.25$ \\
\hline Multijet & $7.8 \pm 0.9$ & $7.3 \pm 0.9$ & $2.50 \pm 0.55$ & $0.78 \pm 0.37$ \\
\hline Single top & $1.19 \pm 0.02$ & $3.84 \pm 0.04$ & $1.63 \pm 0.03$ & $0.48 \pm 0.02$ \\
\hline$t \bar{t} \rightarrow \ell \ell$ & $0.74 \pm 0.02$ & $3.28 \pm 0.05$ & $1.91 \pm 0.04$ & $0.53 \pm 0.02$ \\
\hline Diboson \& $Z \rightarrow \tau \tau$ & $1.13 \pm 0.05$ & $2.75 \pm 0.09$ & $0.40 \pm 0.03$ & $0.06 \pm 0.03$ \\
\hline Total Bkg. & $74.3 \pm 1.2$ & $70.7 \pm 1.1$ & $23.6 \pm 0.7$ & $6.3 \pm 0.4$ \\
\hline Syst. Unc. & $+6.22-7.69$ & $+7.55-8.23$ & $+2.71-2.82$ & $+0.78-0.81$ \\
\hline$t \bar{t} \rightarrow \ell+$ jets & $0.16 \pm 0.02$ & $2.91 \pm 0.10$ & $11.6 \pm 0.2$ & $17.3 \pm 0.2$ \\
\hline Total Expected & $74.5 \pm 1.2$ & $73.6 \pm 1.2$ & $35.2 \pm 0.7$ & $23.6 \pm 0.5$ \\
\hline Syst. Unc. & $+6.22-7.72$ & $+7.67-8.31$ & $+2.89-3.18$ & $+2.24-2.81$ \\
\hline Observed & 68 & 62 & 43 & 24 \\
\hline
\end{tabular}

Table 8.11: Summary of observed and predicted number of single tagged events in the $e+$ jets and the $\mu+$ jets channels. Diboson processes includes $W W, W Z$ and $Z Z$ production. Unless explicitly stated, uncertainties are statistical only. 


\begin{tabular}{|c|c|c|c|c|}
\hline & 1 jet & 2 jets & 3 jets & $\geq 4$ jets \\
\hline & \multicolumn{4}{|c|}{ e+jets channel } \\
\hline$\overline{W+\text { light }}$ & $36.8 \pm 0.7$ & $21.4 \pm 0.7$ & $7.2 \pm 0.4$ & $\overline{1.80 \pm 0.17}$ \\
\hline$W(c \bar{c})$ & $12.2 \pm 0.2$ & $7.3 \pm 0.2$ & $2.52 \pm 0.10$ & $0.81 \pm 0.08$ \\
\hline$W(b \bar{b})$ & $33.9 \pm 0.4$ & $20.3 \pm 0.4$ & $6.4 \pm 0.2$ & $1.95 \pm 0.19$ \\
\hline$W c$ & $47.8 \pm 0.6$ & $24.2 \pm 0.5$ & $5.7 \pm 0.2$ & $0.79 \pm 0.08$ \\
\hline$W c \bar{c}$ & & $9.9 \pm 0.2$ & $4.09 \pm 0.18$ & $1.39 \pm 0.13$ \\
\hline$W b \bar{b}$ & & $22.9 \pm 0.4$ & $8.6 \pm 0.3$ & $2.58 \pm 0.24$ \\
\hline$\overline{W+\text { jets }}$ & $130.8 \pm 1.1$ & $106.0 \pm 1.1$ & $34.5 \pm 0.6$ & $9.3 \pm 0.4$ \\
\hline Multijet & $14.9 \pm 1.9$ & $16.3 \pm 2.1$ & $8.3 \pm 1.5$ & $4.05 \pm 1.21$ \\
\hline Single top & $2.65 \pm 0.03$ & $8.11 \pm 0.06$ & $3.41 \pm 0.04$ & $2.01 \pm 0.02$ \\
\hline$t \bar{t} \rightarrow \ell \ell$ & $1.75 \pm 0.04$ & $7.1 \pm 0.1$ & $3.95 \pm 0.06$ & $1.09 \pm 0.03$ \\
\hline Diboson \& $Z \rightarrow \tau \tau$ & $2.21 \pm 0.07$ & $5.33 \pm 0.11$ & $0.81 \pm 0.04$ & $0.11 \pm 0.03$ \\
\hline Total Bkg. & $152.4 \pm 2.1$ & $142.9 \pm 2.3$ & $51.0 \pm 1.6$ & $15.6 \pm 1.3$ \\
\hline Syst. Unc. & $+13.16-16.41$ & $+15.31-16.64$ & $+5.46-5.72$ & $+1.65-1.71$ \\
\hline$\overline{t \bar{t}} \rightarrow \ell+$ jets & $0.40 \pm 0.04$ & $6.8 \pm 0.2$ & $24.4 \pm 0.3$ & $34.8 \pm 0.3$ \\
\hline Total Expected & $152.8 \pm 2.1$ & $149.7 \pm 2.3$ & $75.4 \pm 1.6$ & $50.4 \pm 1.3$ \\
\hline Syst. Unc. & $+13.18-16.43$ & $+15.54-16.79$ & $+5.72-6.10$ & $+4.38-5.12$ \\
\hline Observed & 137 & 145 & 79 & 62 \\
\hline
\end{tabular}

Table 8.12: Summary of observed and predicted number of single tagged events in the combined $\ell+$ jets channel. Diboson processes includes $W W, W Z$ and $Z Z$ production. Unless explicitly stated, uncertainties are statistical only. 


\begin{tabular}{|c|c|c|c|}
\hline & 2 jets & 3 jets & $\geq 4$ jets \\
\hline & \multicolumn{3}{|c|}{$e+$ +jets channel } \\
\hline $\bar{~} W+$ light & $\overline{0.012 \pm 0.002}$ & $0.011 \pm 0.001$ & $<00.01$ \\
\hline$W(c \bar{c})$ & $0.013 \pm 0.002$ & $<0.01$ & $<0.01$ \\
\hline$W(b \bar{b})$ & $0.14 \pm 0.02$ & $0.07 \pm 0.01$ & $0.02 \pm 0.01$ \\
\hline$W c$ & $0.023 \pm 0.001$ & $0.011 \pm 0.001$ & $<0.01$ \\
\hline$W c \bar{c}$ & $0.19 \pm 0.01$ & $0.08 \pm 0.01$ & $0.04 \pm 0.01$ \\
\hline$W b \bar{b}$ & $2.66 \pm 0.10$ & $0.99 \pm 0.07$ & $0.32 \pm 0.05$ \\
\hline$W+$ jets & $3.05 \pm 0.10$ & $1.18 \pm 0.07$ & $0.39 \pm 0.05$ \\
\hline Multijet & $<0.01$ & $0.08 \pm 0.23$ & $<0.01$ \\
\hline Single top & $0.55 \pm 0.01$ & $0.32 \pm 0.01$ & $0.11 \pm 0.01$ \\
\hline$t \bar{t} \rightarrow \ell \ell$ & $0.96 \pm 0.02$ & $0.55 \pm 0.01$ & $0.16 \pm 0.01$ \\
\hline Diboson \& $Z \rightarrow \tau \tau$ & $0.26 \pm 0.01$ & $0.04 \pm 0.01$ & $<0.01$ \\
\hline Total Bkg. & $4.74 \pm 0.20$ & $2.19 \pm 0.24$ & $0.67 \pm 0.28$ \\
\hline Syst. Unc. & $+0.91-0.90$ & $+0.38-0.37$ & $+0.12-0.13$ \\
\hline$t \bar{t} \rightarrow \ell+$ jets & $0.49 \pm 0.02$ & $3.09 \pm 0.06$ & $5.6 \pm 0.1$ \\
\hline Total Expected & $5.2 \pm 0.2$ & $5.3 \pm 0.3$ & $6.2 \pm 0.3$ \\
\hline Syst. Unc. & $+0.94-0.93$ & $+0.64-0.58$ & $+0.99-1.05$ \\
\hline \multirow[t]{2}{*}{ Observed } & 6 & 6 & 9 \\
\hline & \multicolumn{3}{|c|}{$\begin{array}{r}\mu+\text { jets channel } \\
\end{array}$} \\
\hline $\bar{W} W+$ light & $0.016 \pm 0.002$ & $0.010 \pm 0.001$ & $<<0.01$ \\
\hline$W(c \bar{c})$ & $0.014 \pm 0.002$ & $<0.01$ & $<0.01$ \\
\hline$W(b \bar{b})$ & $0.19 \pm 0.03$ & $0.064 \pm 0.005$ & $0.03 \pm 0.01$ \\
\hline$W c$ & $0.028 \pm 0.002$ & $0.012 \pm 0.001$ & $<0.01$ \\
\hline$W c \bar{c}$ & $0.22 \pm 0.01$ & $0.09 \pm 0.01$ & $0.031 \pm 0.004$ \\
\hline$W b \bar{b}$ & $2.74 \pm 0.09$ & $1.02 \pm 0.06$ & $0.28 \pm 0.04$ \\
\hline$W+$ jets & $3.20 \pm 0.09$ & $1.21 \pm 0.06$ & $0.36 \pm 0.04$ \\
\hline Multijet & $<0.01$ & $<0.01$ & $<0.01$ \\
\hline Single top & $0.50 \pm 0.01$ & $0.29 \pm 0.01$ & $0.10 \pm 0.01$ \\
\hline$t \bar{t} \rightarrow \ell \ell$ & $0.81 \pm 0.02$ & $0.51 \pm 0.01$ & $0.15 \pm 0.01$ \\
\hline Diboson \& $Z \rightarrow \tau \tau$ & $0.26 \pm 0.01$ & $0.03 \pm 0.01$ & $<0.01$ \\
\hline Total Bkg. & $4.66 \pm 0.10$ & $2.00 \pm 0.07$ & $0.50 \pm 0.06$ \\
\hline Syst. Unc. & $+0.91-0.93$ & $+0.39-0.36$ & $+0.12-0.13$ \\
\hline$t \bar{t} \rightarrow \ell+$ jets & $0.33 \pm 0.02$ & $2.79 \pm 0.06$ & $5.3 \pm 0.1$ \\
\hline Total Expected & $4.98 \pm 0.10$ & $4.79 \pm 0.09$ & $5.8 \pm 0.1$ \\
\hline Syst. Unc. & $+0.95-0.94$ & $+0.61-0.63$ & $+0.95-1.10$ \\
\hline Observed & 9 & 3 & 5 \\
\hline
\end{tabular}

Table 8.13: Summary of observed and predicted number of double tagged events in the $e+$ jets and the $\mu+$ jets channels. Diboson processes includes $W W, W Z$ and $Z Z$ production. Unless explicitly stated, uncertainties are statistical only. 


\begin{tabular}{l|ccc}
\hline \hline \multicolumn{4}{c}{$\ell+$ jets channel } \\
\hline \hline \multicolumn{4}{c}{2 jets } \\
\hline \hline$W+$ light & $0.027 \pm 0.002$ & $0.021 \pm 0.002$ & $<0.01$ \\
$W(c \bar{c})$ & $0.027 \pm 0.002$ & $0.019 \pm 0.001$ & $<0.01$ \\
$W(b \bar{b})$ & $0.33 \pm 0.03$ & $0.14 \pm 0.01$ & $0.05 \pm 0.01$ \\
$W c$ & $0.050 \pm 0.002$ & $0.023 \pm 0.001$ & $<0.01$ \\
$W c \bar{c}$ & $0.41 \pm 0.01$ & $0.17 \pm 0.01$ & $0.07 \pm 0.01$ \\
$W b \bar{b}$ & $5.4 \pm 0.1$ & $2.02 \pm 0.09$ & $0.61 \pm 0.06$ \\
\hline$W+$ jets & $6.2 \pm 0.1$ & $2.39 \pm 0.09$ & $0.75 \pm 0.06$ \\
\hline Multijet & $<0.01$ & $0.04 \pm 0.23$ & $<0.01$ \\
\hline Single top & $1.05 \pm 0.02$ & $0.51 \pm 0.01$ & $0.21 \pm 0.01$ \\
$t \bar{t} \rightarrow \ell \ell$ & $1.78 \pm 0.02$ & $1.06 \pm 0.02$ & $0.31 \pm 0.01$ \\
Diboson \& $Z \rightarrow \tau \tau$ & $0.52 \pm 0.02$ & $0.08 \pm 0.01$ & $<0.01$ \\
\hline Total Bkg. & $9.4 \pm 0.2$ & $4.19 \pm 0.27$ & $1.17 \pm 0.31$ \\
\hline Syst. Unc. & $+1.82-1.80$ & $+0.77-0.74$ & $+0.24-0.26$ \\
\hline$t \bar{t} \rightarrow \ell+$ jets & $0.82 \pm 0.03$ & $5.9 \pm 0.1$ & $10.9 \pm 0.1$ \\
\hline Total Expected & $10.2 \pm 0.2$ & $10.1 \pm 0.3$ & $12.1 \pm 0.3$ \\
\hline Syst. Unc. & $+1.88-1.85$ & $+1.23-1.17$ & $+1.95-2.08$ \\
\hline Observed & 15 & 9 & 14 \\
\hline \hline
\end{tabular}

Table 8.14: Summary of observed and predicted number of double tagged events in the combined $\ell+$ jets channel. Diboson processes includes $W W, W Z$ and $Z Z$ production. Unless explicitly stated, uncertainties are statistical only. 

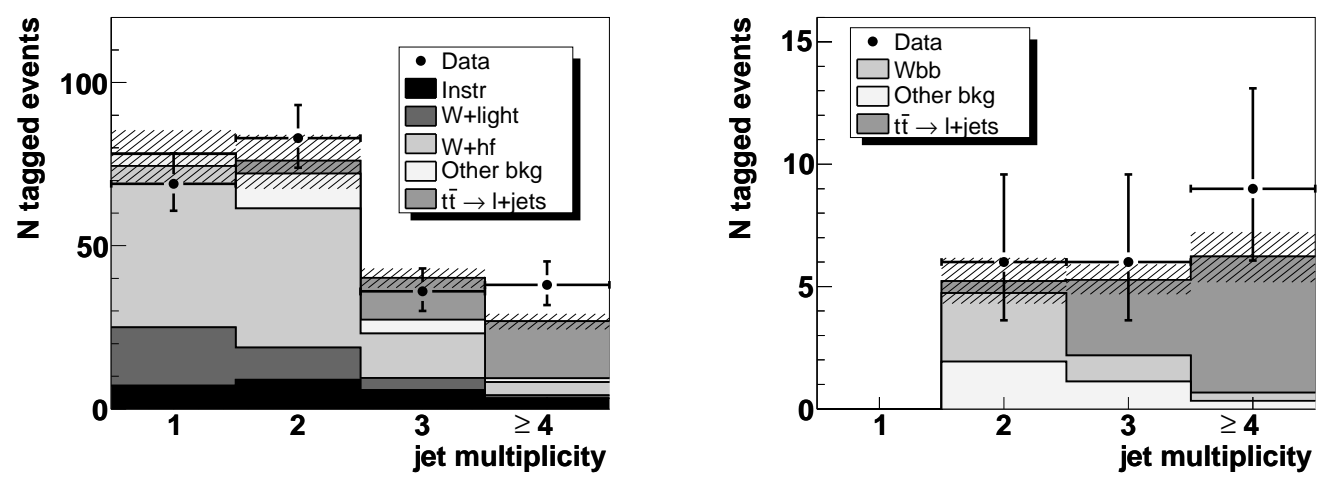

Figure 8.1: Summary plots of observed and predicted number of tagged events in the $e+$ jets channel. The left plot shows single tagged events and the right plot shows double tagged events. The total uncertainty on the signal plus background prediction is represented by the dashed band.

\subsection{Kinematic Distributions}

The kinematic distributions of events in the preselected sample are compared with the expected kinematic distributions for the sum of signal and background events. The measured $t \bar{t}$ cross section is used to calculate the number of $t \bar{t}$ events. The comparison is made in all jet multiplicity bins. Furthermore, it is made for preselected events before tagging is applied, for single tagged events and for double tagged events separately. The various distributions are shown in Figs. 8.4-8.11. The eight kinematic variables shown are:

- Missing transverse energy $\not_{T}$.

- $H_{T}$, defined as the scalar sum of the $p_{T}$ of the jets in the event.

- $H_{T 2}^{\prime}$, defined as $H_{T 2} / H_{z}$ where $H_{T 2}$ is the scalar sum of the $p_{T}$ for all jets excluding the leading jet and $H_{z}$ is the scalar sum of $\left|p_{z}\right|$ of all the jets plus the absolute value of the momentum of the lepton and the neutrino along the $z$-direction ${ }^{1}$.

- The sphericity $\mathcal{S}$ of the event, defined as

$$
\mathcal{S}=\frac{3}{2}\left(\lambda_{2}+\lambda_{3}\right)
$$

\footnotetext{
${ }^{1}$ The neutrino momentum along the $z$ direction, $p_{z}^{\nu}$, is determined by assuming that each event contains one $W$ boson. The invariant mass of the $W$ boson is used to derive $p_{z}^{\nu}$. The solution with the smallest $\left|p_{z}^{\nu}\right|$ is chosen.
} 

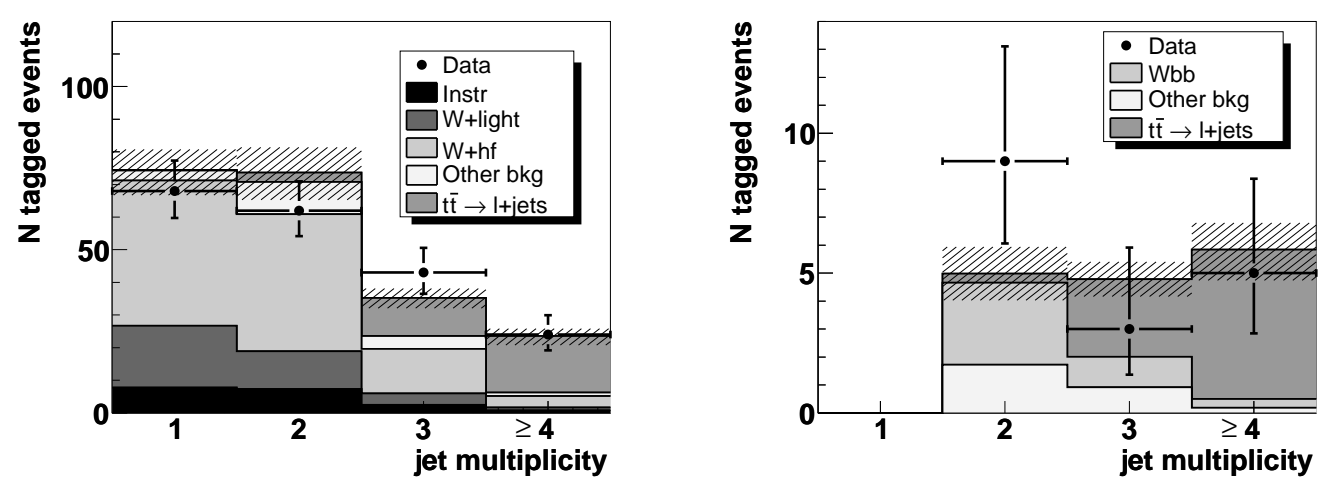

Figure 8.2: Summary plots of observed and predicted number of tagged events in the $\mu+$ jets channel. The left plot shows single tagged events and the right plot shows double tagged events. The total uncertainty on the background prediction is represented by the dashed band.

where $\lambda_{2}$ and $\lambda_{3}$ are the smallest eigenvalues of the normalized momentum tensor $\mathcal{M}$

$$
\mathcal{M}_{i j}=\frac{\Sigma_{o} p_{i}^{o} p_{j}^{o}}{\Sigma_{o}\left|\overrightarrow{p^{o}}\right|^{2}}
$$

so that $0 \leq \mathcal{S} \leq 1$. Sphericity corresponds to the sum of $p_{\perp}^{2}$ with respect to the event axis, a two-jet event will have $\mathcal{S} \approx 0$ and an isotropic event $\mathcal{S} \approx 1$. Due to the large mass of the top quarks, $t \bar{t}$ events typically have large values of sphericity. $W+$ jets and multijet events, where the jets are primarily created from initial state radiation, are expected to be less isotropic.

- Aplanarity $\mathcal{A}$, defined as

$$
\mathcal{A}=\frac{3}{2} \lambda_{3}
$$

where $\lambda_{3}$ is the smallest eigenvalue of the normalized momentum tensor defined in Eq. 8.9.

- The centrality $\mathrm{C}=H_{T} / H . H_{T}$ is given by the scalar sum of the $p_{T}$ of the jets in the event, and $H$ is the scalar sum of the $p$ of the jets.

- $K_{T m i n}^{\prime}=\Delta \mathcal{R}_{j j}^{\min } p_{T}^{\min } / p_{T}^{W}$ is the product of the minimum di-jet separation in $\Delta \mathcal{R}$ and the $p_{T}$ of the less energetic jet of the pair, divided by the scalar sum of the $p_{T}$ of the lepton and the $\mathscr{E}_{T}$.

- Topological likelihood discriminant for $t \bar{t}$ events. This variable is described in more detail in Sec. 9.2.2. 

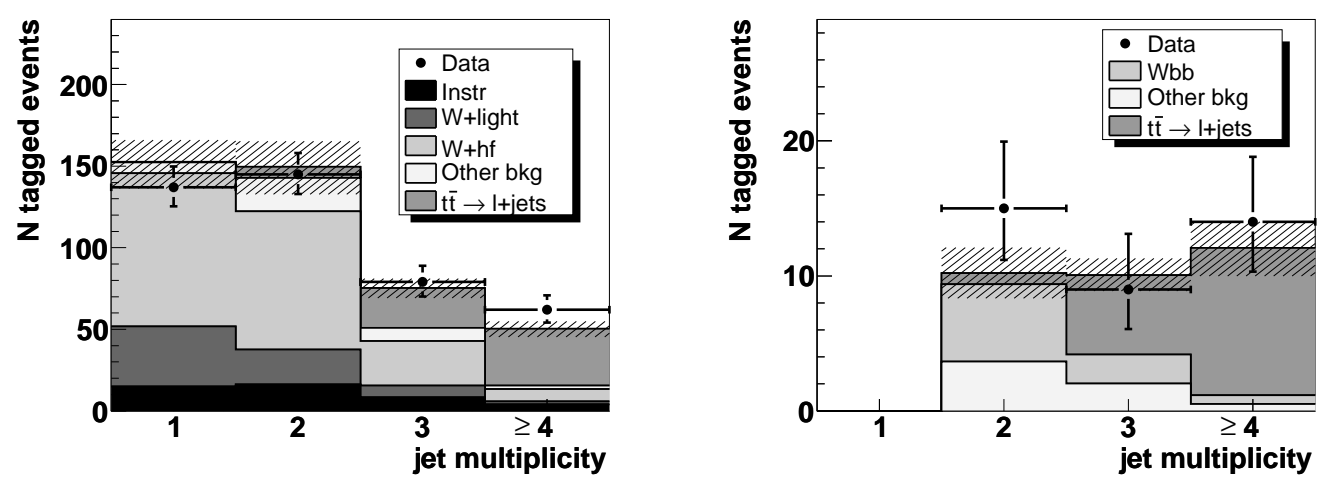

Figure 8.3: Summary plots of observed and predicted number of tagged events in the combined $\ell+$ jets channel. The left plot shows single tagged events and the right plot shows double tagged events. The total uncertainty on the background prediction is represented by the dashed band.

The shapes of all distributions in data are reasonably well described by the sum of signal plus background contributions. No uncertainties are shown for the prediction. 

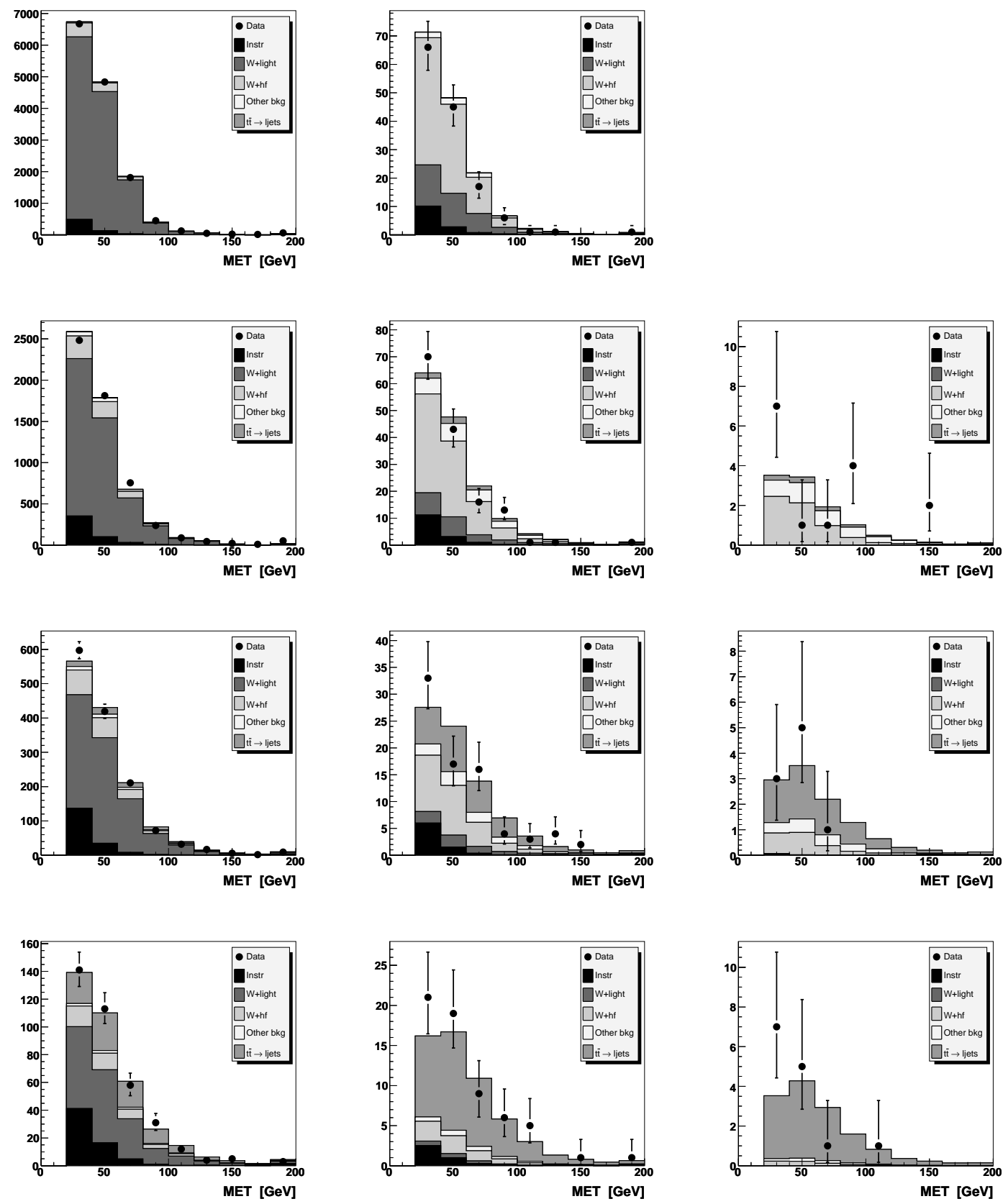

All Preselected Events

Single Tagged Events

Double Tagged Events

Figure 8.4: $\mathbb{F}_{T}$ distributions for events in the $\ell+$ jets preselected sample. The points correspond to the observation and the histogram to the prediction from signal plus background. The top row corresponds to events with 1 jet, the second row to events with 2 jets, the third row to events with 3 jets and the bottom row to events with $\geq 4$ jets. The left column corresponds to all events passing the preselection criteria. The middle column corresponds to all single tagged events and the right column to all double tagged events. The prediction is shown without uncertainties. 

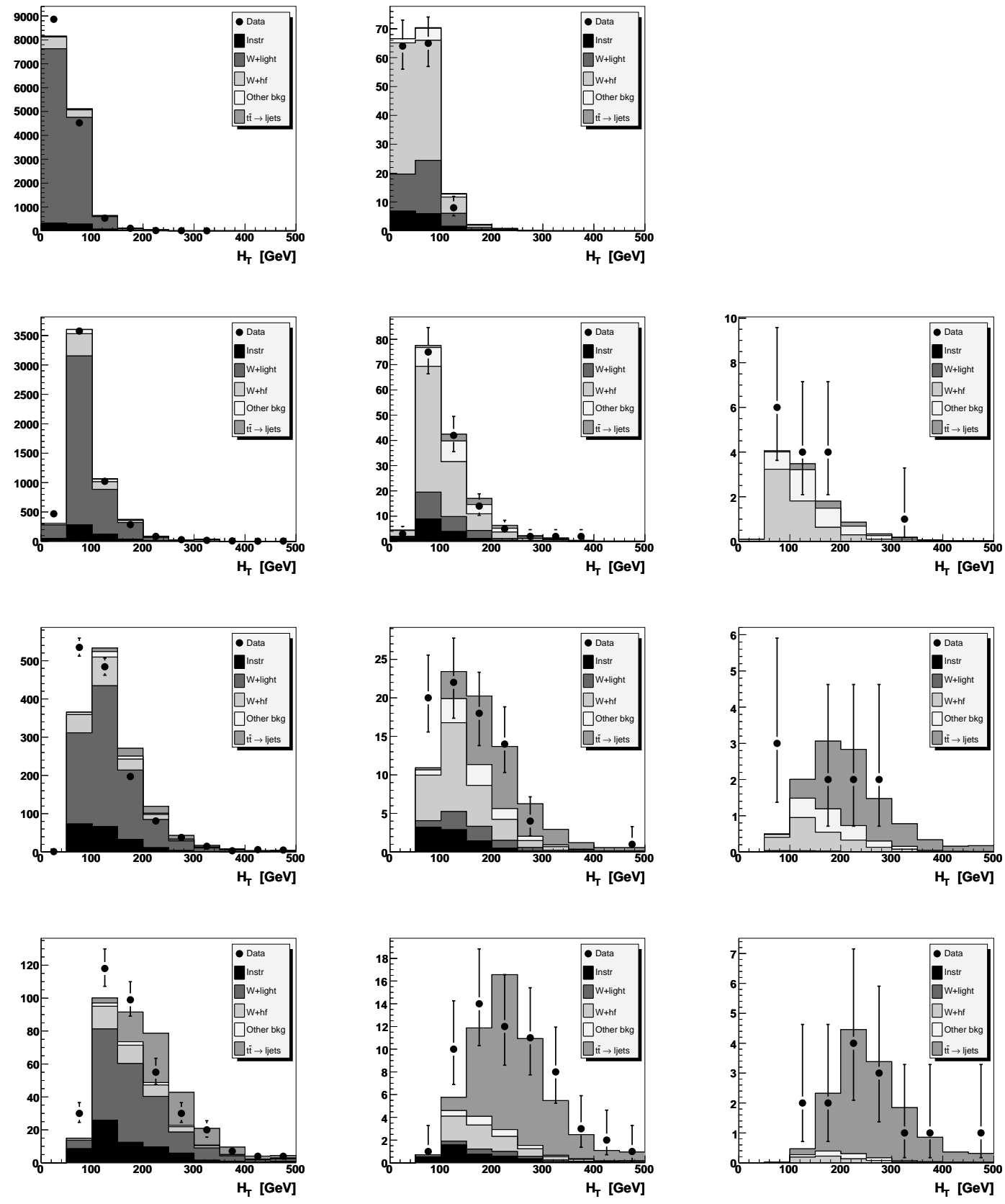

All Preselected Events

Single Tagged Events

Double Tagged Events

Figure 8.5: $H_{T}$ distributions for events in the $\ell+$ jets preselected sample. The points correspond to the observation and the histogram to the prediction from signal plus background. The top row corresponds to events with 1 jet, the second row to events with 2 jets, the third row to events with 3 jets and the bottom row to events with $\geq 4$ jets. The left column corresponds to all events passing the preselection criteria. The middle column corresponds to all single tagged events and the right column to all double tagged events. The prediction is shown without uncertainties. 

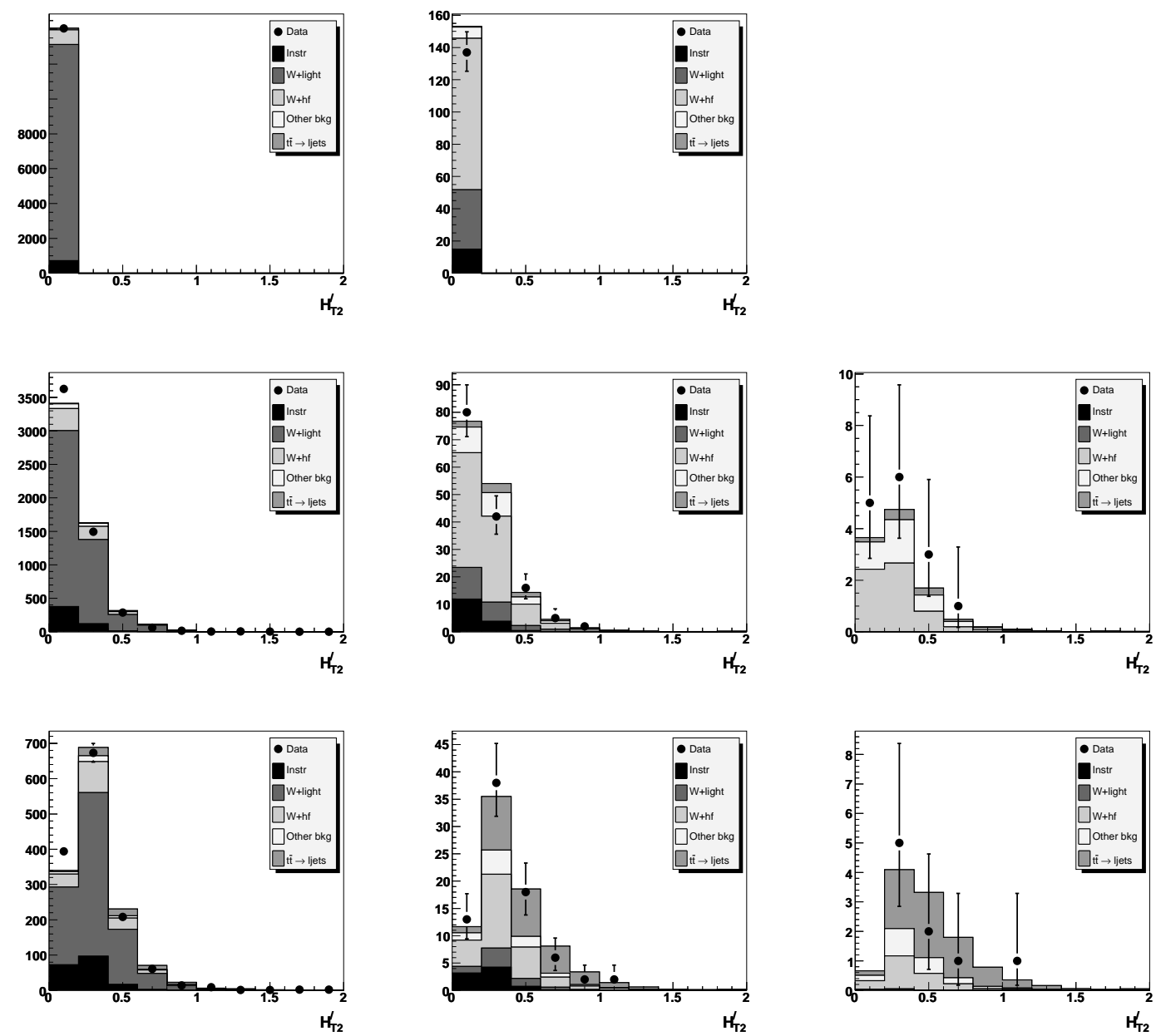

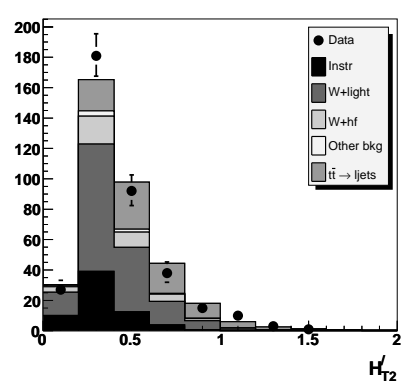

All Preselected Events

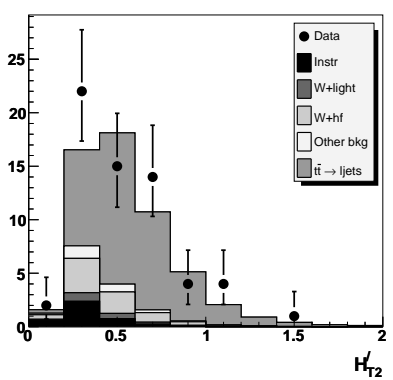

Single Tagged Events

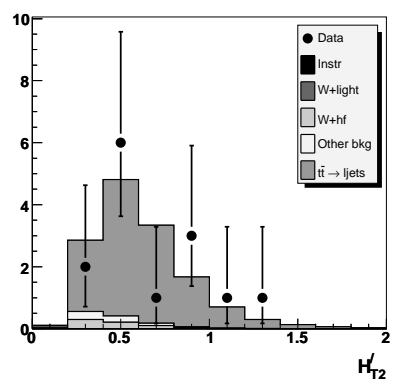

Double Tagged Events

Figure 8.6: $H_{T 2}^{\prime}$ distributions for events in the $\ell+$ jets preselected sample. The points correspond to the observation and the histogram to the prediction from signal plus background. The top row corresponds to events with 1 jet, the second row to events with 2 jets, the third row to events with 3 jets and the bottom row to events with $\geq 4$ jets. The left column corresponds to all events passing the preselection criteria. The middle column corresponds to all single tagged events and the right column to all double tagged events. The prediction is shown without uncertainties. 

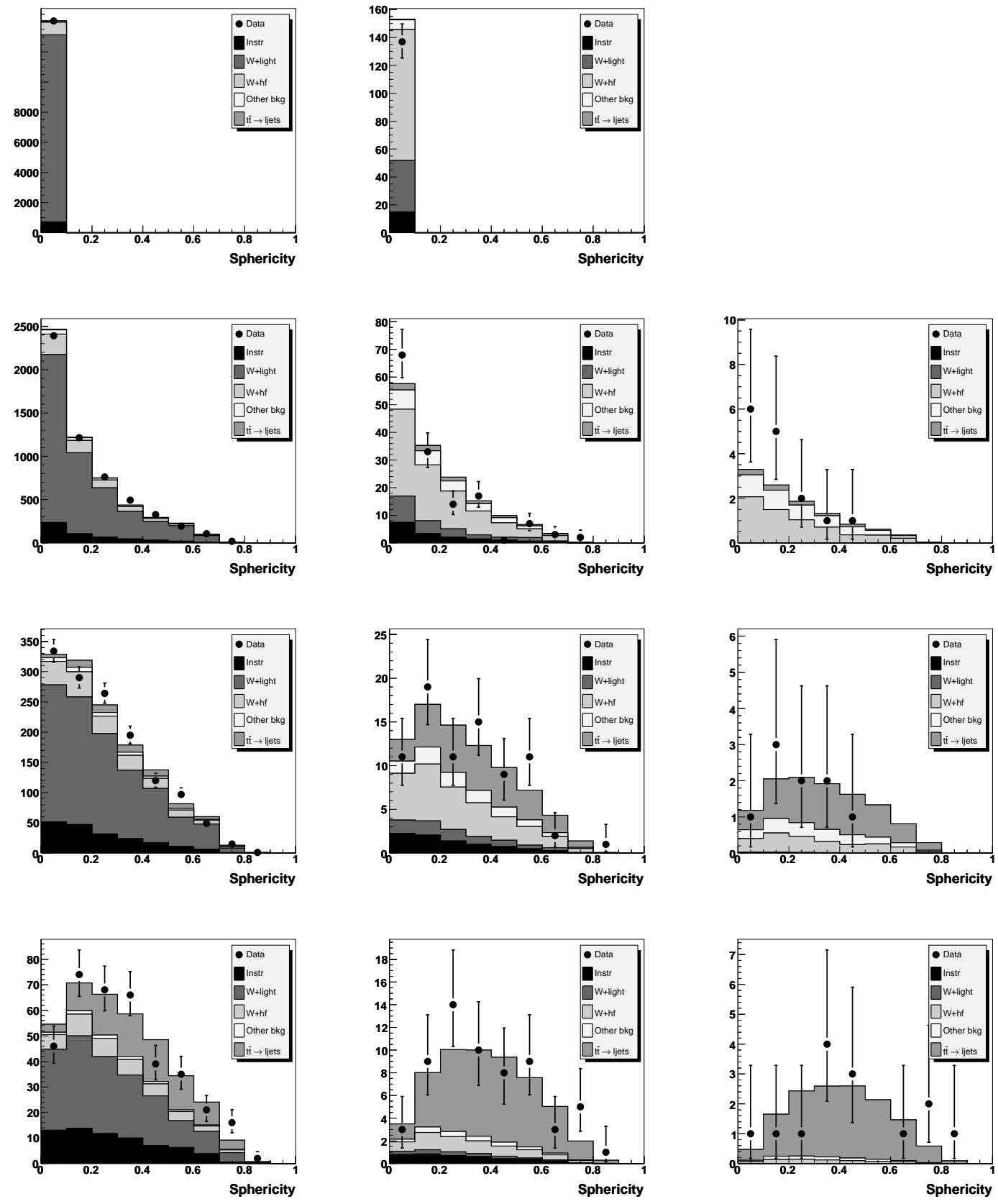

All Preselected Events

Single Tagged Events

Double Tagged Events

Figure 8.7: Sphericity distributions for events in the $\ell+$ jets preselected sample. The points correspond to the observation and the histogram to the prediction from signal plus background. The top row corresponds to events with 1 jet, the second row to events with 2 jets, the third row to events with 3 jets and the bottom row to events with $\geq 4$ jets. The left column corresponds to all events passing the preselection criteria. The middle column corresponds to all single tagged events and the right column to all double tagged events. The prediction is shown without uncertainties. 

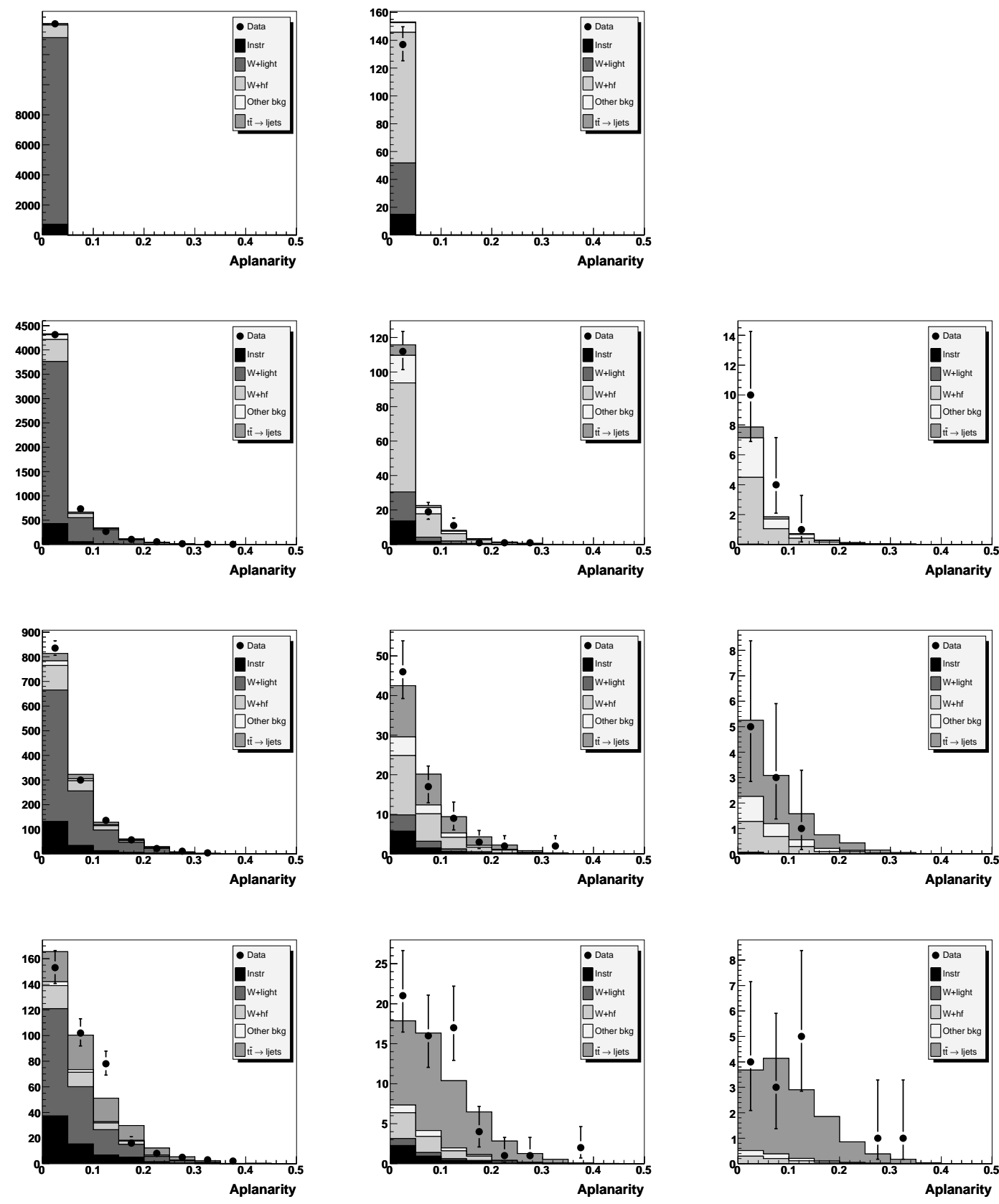

All Preselected Events

Single Tagged Events

Double Tagged Events

Figure 8.8: Aplanarity distributions for events in the $\ell+$ jets preselected sample. The points correspond to the observation and the histogram to the prediction from signal plus background. The top row corresponds to events with 1 jet, the second row to events with 2 jets, the third row to events with 3 jets and the bottom row to events with $\geq 4$ jets. The left column corresponds to all events passing the preselection criteria. The middle column corresponds to all single tagged events and the right column to all double tagged events. The prediction is shown without uncertainties. 

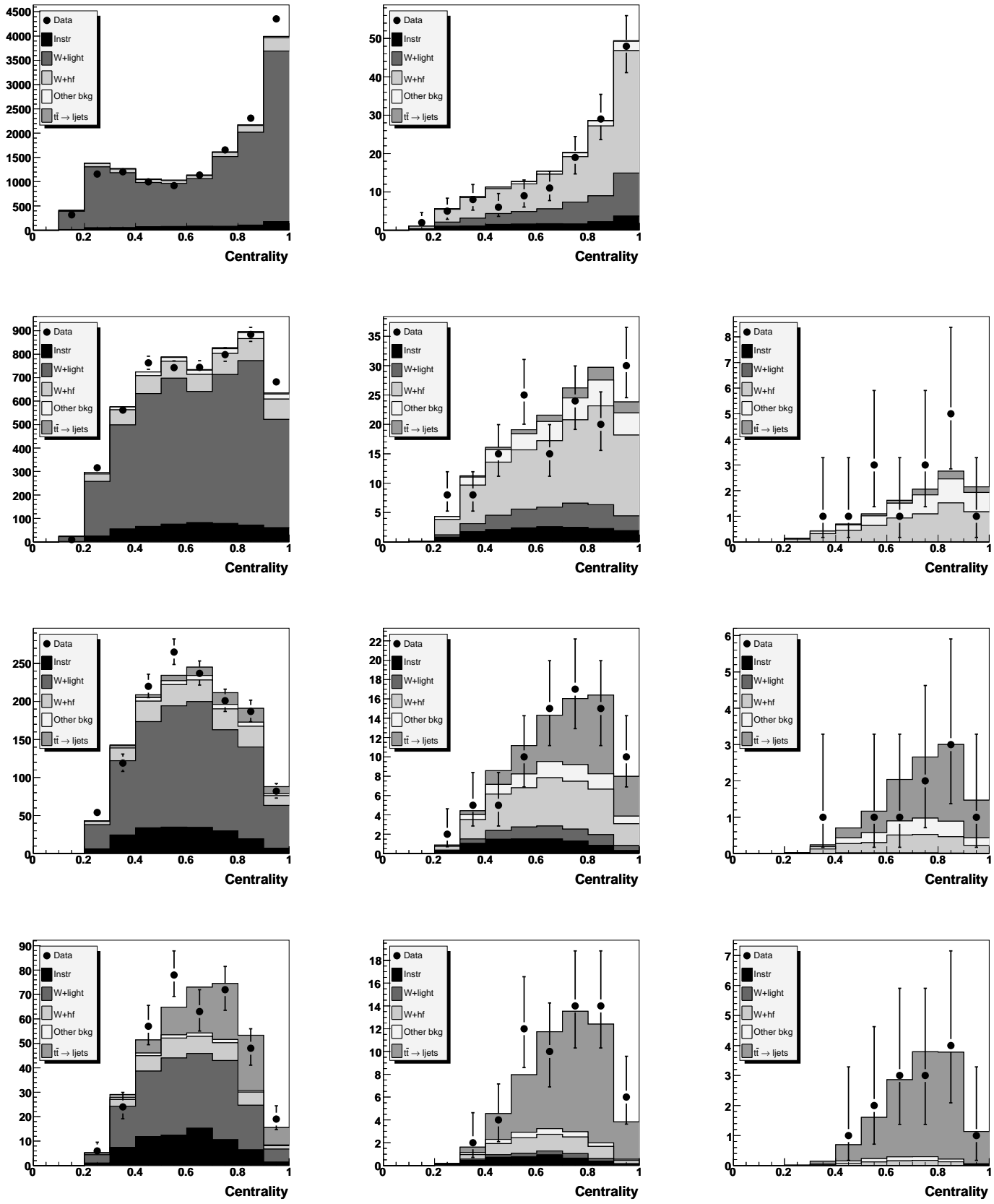

All Preselected Events

Single Tagged Events

Double Tagged Events

Figure 8.9: Centrality distributions for events in the $\ell+$ jets preselected sample. The points correspond to the observation and the histogram to the prediction from signal plus background. The top row corresponds to events with 1 jet, the second row to events with 2 jets, the third row to events with 3 jets and the bottom row to events with $\geq 4$ jets. The left column corresponds to all events passing the preselection criteria. The middle column corresponds to all single tagged events and the right column to all double tagged events. The prediction is shown without uncertainties. 

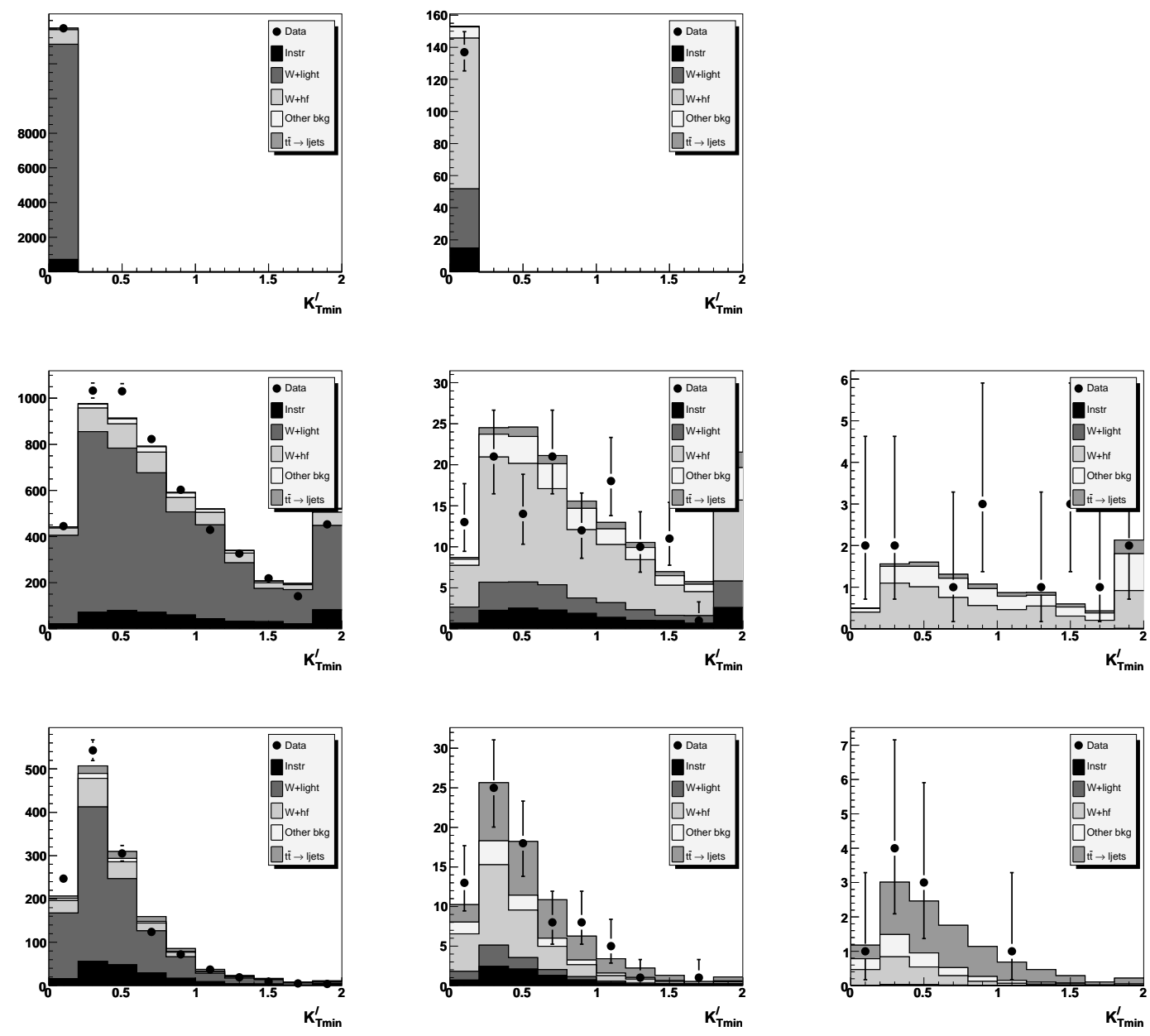

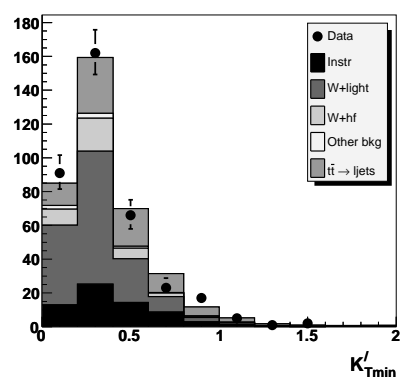

All Preselected Events

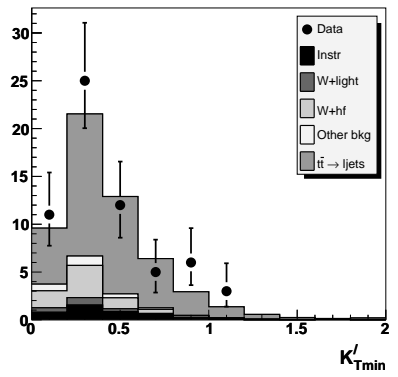

Single Tagged Events

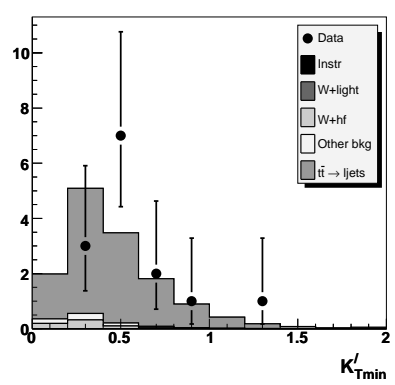

Double Tagged Events

Figure 8.10: $K_{\text {Tmin }}^{\prime}$ distributions for events in the $\ell+$ jets preselected sample. The points correspond to the observation and the histogram to the prediction from signal plus background. The top row corresponds to events with 1 jet, the second row to events with 2 jets, the third row to events with 3 jets and the bottom row to events with $\geq 4$ jets. The left column corresponds to all events passing the preselection criteria. The middle column corresponds to all single tagged events and the right column to all double tagged events. The prediction is shown without uncertainties. 

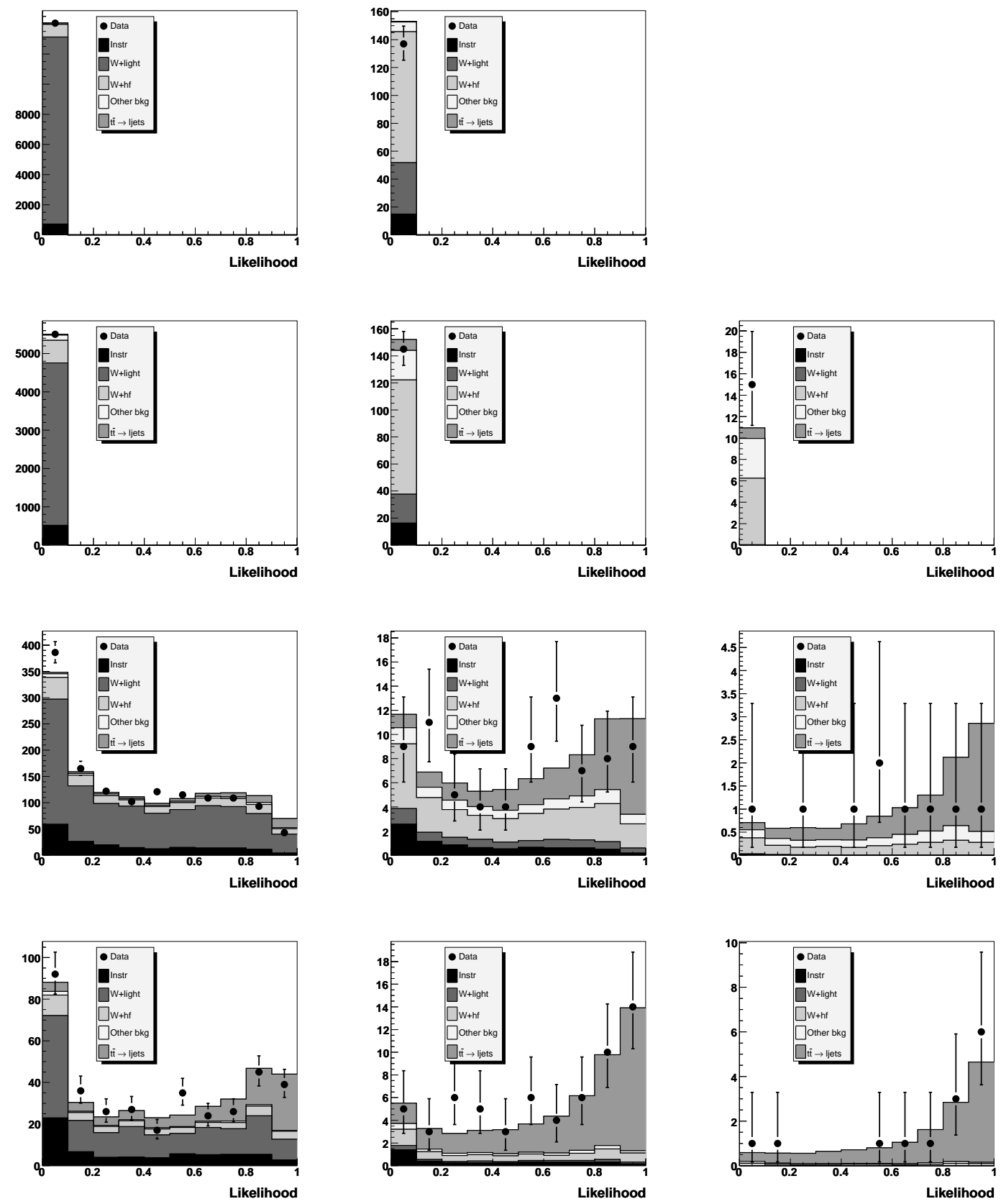

All Preselected Events

Single Tagged Events

Double Tagged Events

Figure 8.11: Topological likelihood distributions for events in the $\ell+$ jets preselected sample. The points correspond to the observation and the histogram to the prediction from signal plus background. The top row corresponds to events with 1 jet, the second row to events with 2 jets, the third row to events with 3 jets and the bottom row to events with $\geq 4$ jets. The left column corresponds to all events passing the preselection criteria. The middle column corresponds to all single tagged events and the right column to all double tagged events. The prediction is shown without uncertainties. 


\section{Chapter 9}

\section{Results}

This chapter describes how the number of observed events in the $b$-tagged sample is used to perform two measurements. The measurement in Paper I interprets the excess of observed single and double tagged events over the expected background in terms of a $t \bar{t}$ production cross section $\sigma_{t \bar{t}}$. The measurement in Paper II is an extension of the cross section measurement. A technique to constrain the number of $t \bar{t}$ events present in the sample without $b$-tags is first developed. The number of $t \bar{t}$ events with 0,1 and $2 b$-tagged jets are then used to simultaneously measure the $t \bar{t}$ production cross section $\sigma_{t \bar{t}}$ and the ratio $R$,

$$
R=\frac{B(t \rightarrow W b)}{B(t \rightarrow W q)}
$$

where $q$ is any down-type quark.

\subsection{Measurement of $\sigma_{t \bar{t}}$}

The excess of observed $b$-tagged events over the predicted number of background events in the third and fourth jet multiplicity bins is interpreted as coming from $t \bar{t}$ production. The production cross section is related to the number of observed $t \bar{t}$ events $N_{t \bar{t}}$ through:

$$
N_{t \bar{t}}=L \cdot \sigma_{t \bar{t}} \cdot B(t \bar{t} \rightarrow \ell+\mathrm{jets}) \cdot \epsilon_{t \bar{t}}^{\text {presel }} \cdot \epsilon_{t \bar{t}}^{\text {tagging }}
$$

In the $\sigma_{t \bar{t}}$ measurement, each combination of third and fourth jet multiplicity bin, single and double tagged events, and $e+$ jets and $\mu+$ jets channel is treated as a separate channel. In total there are eight independent channels, as shown in Tab. 9.1.

Each preselection efficiency and event tagging probability has a statistical uncertainty assigned to it due to the limited statistics in the simulated samples where they are evaluated. These statistical uncertainties are uncorrelated between all jet multiplicity bins, the $e+$ jets and $\mu+$ jets channels and single tagged and double 


\begin{tabular}{|l|c|c|c|c|}
\hline & \multicolumn{2}{|c|}{$e+$ jets channel } & \multicolumn{2}{c|}{$\mu+$ jets channel } \\
\hline & Single Tags & Double Tags & Single Tags & Double Tags \\
\hline 3 jets & $\times$ & $\times$ & $\times$ & $\times$ \\
\hline$\geq 4$ jets & $\times$ & $\times$ & $\times$ & $\times$ \\
\hline
\end{tabular}

Table 9.1: Illustration of the eight independent channels in the $t \bar{t}$ cross section measurement.
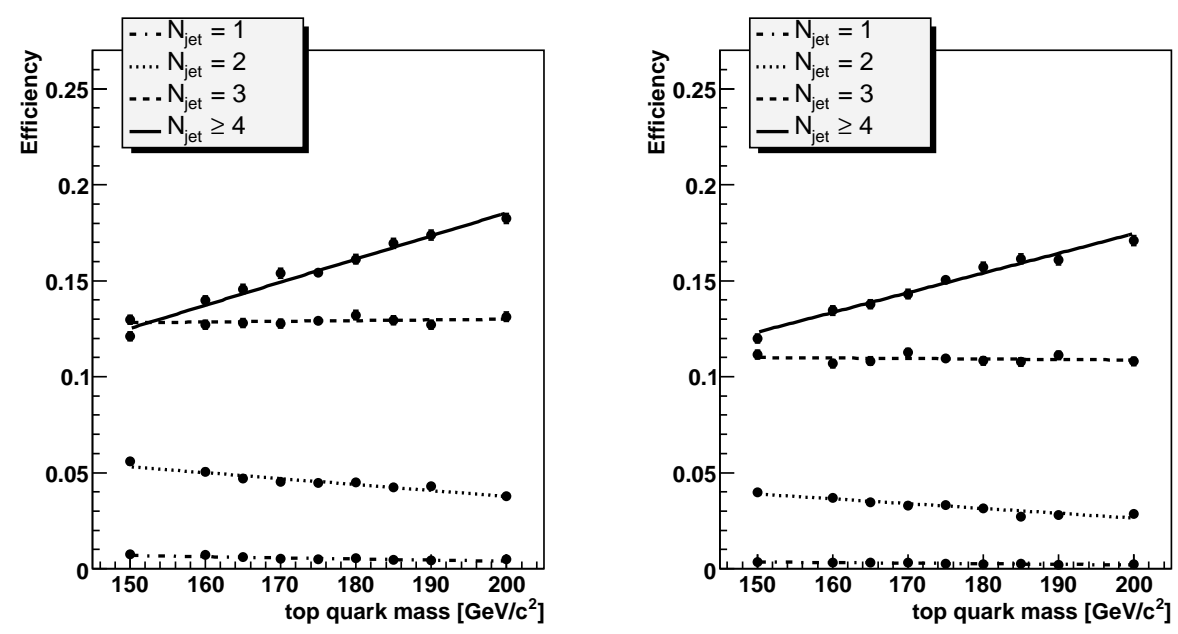

Figure 9.1: The mass dependence of the $t \bar{t}$ preselection efficiency in the $e+$ jets (left plot) and $\mu+$ jets (right plot) channels.

tagged events. In addition there are systematic uncertainties which are correlated between some or all of the eight independent channels. The systematic uncertainties considered in the cross section measurement are described in Sec. 9.1.1 below. The uncertainties from limited statistics in the simulated samples are in general small compared to the systematic uncertainties.

The mass of the top quark influences both the preselection efficiency and the tagging probability for $t \bar{t}$ events. Several $t \bar{t}$ samples have been generated for top masses of $150,160,165,170,175,180,185,190$ and $200 \mathrm{GeV} / c^{2}$. Figure 9.1 shows the mass dependence of the preselection efficiency, and Fig. 9.2 shows the mass dependence of the event tagging probability. The top quark mass is not treated as a source of systematic uncertainty. Instead the mass dependence of the preselection efficiency and the tagging probability is used to derive the $t \bar{t}$ production cross section as a function of the top quark mass. 

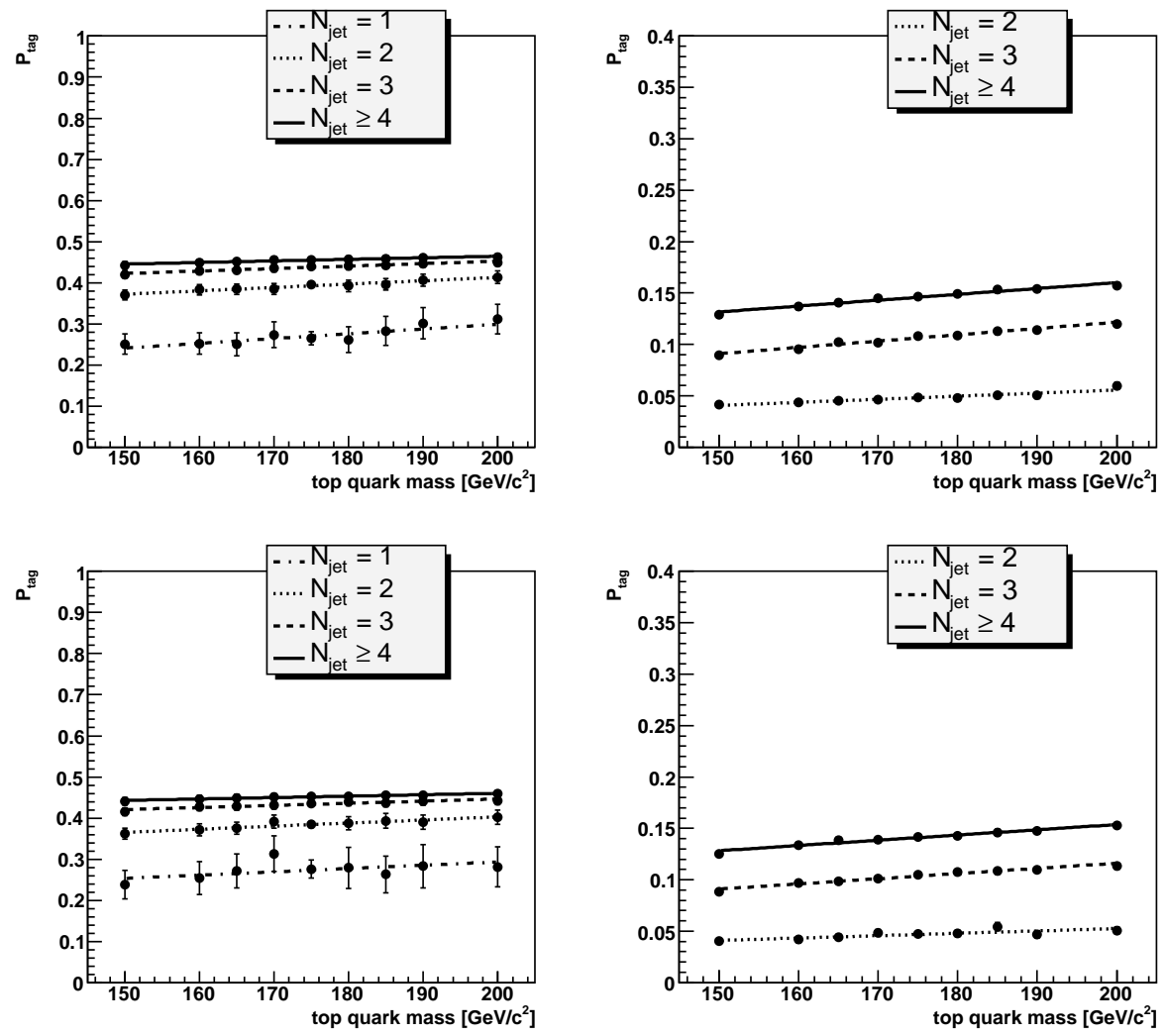

Figure 9.2: The mass dependence of the $t \bar{t}$ event tagging probabilities. The top left plot shows the single tag probability and the top right the double tag probability in the $e+$ jets channel. The lower two plots shows the single tag probability (left plot) and double tag probability (right plot) in the $\mu+$ jets channel. 


\subsubsection{Systematic Uncertainties}

The uncertainty on the integrated luminosity is derived from the measurement of the inelastic cross section for $p \bar{p}$ collisions, described in Ref. [56]. The total uncertainty on the integrated luminosity is $6.5 \%$.

A summary of the systematic uncertainties and how they are correlated is given in Tab. 9.2. Each systematic uncertainty can affect either the background prediction, $N^{b k g}$, the $t \bar{t}$ signal efficiency, $\varepsilon_{t o t}^{t \bar{t}}$, or both. For each systematic uncertainty in Tab. 9.2, the crosses indicates which of $N^{b k g}$ and $\varepsilon_{\text {tot }}^{t \bar{t}}$ is affected, and in which channel. If a systematic uncertainty affects more than one category, the uncertainty is fully correlated between the crossed categories. All systematic uncertainties are fully correlated between the single tagged and the double tagged samples, and between the third and fourth jet multiplicity bins. A description of each systematic uncertainty is given below.

\begin{tabular}{|c|c|c|c|c|}
\hline \hline Source of & \multicolumn{2}{|c|}{$e+$ jets } & \multicolumn{2}{|c|}{$\mu+$ jets } \\
\cline { 2 - 5 } systematic uncertainty & $N^{b k g}$ & $\varepsilon_{\text {tot }}^{t t}$ & $N^{b k g}$ & $\varepsilon_{t o t}^{t t}$ \\
\hline$\varepsilon_{\text {lept }}$ and $\varepsilon_{\text {instr }}$ in $e+$ jets channel & $\times$ & & & \\
$\varepsilon_{\text {lept }}$ and $\varepsilon_{\text {instr } \text { in } \mu+\text { jets channel }}$ & & & $\times$ & \\
Muon data-to-simulation corrections & & & $\times$ & $\times$ \\
Muon trigger requirements & & & $\times$ & $\times$ \\
Electron data-to-simulation corrections & $\times$ & $\times$ & & \\
Electron trigger requirements & $\times$ & $\times$ & & \\
Jet energy scale & $\times$ & $\times$ & $\times$ & $\times$ \\
Jet energy resolution & $\times$ & $\times$ & $\times$ & $\times$ \\
Jet reconstruction and identification & $\times$ & $\times$ & $\times$ & $\times$ \\
Jet trigger requirements & $\times$ & $\times$ & $\times$ & $\times$ \\
Taggability parameterizations & $\times$ & $\times$ & $\times$ & $\times$ \\
$b$-tagging efficiency in data & $\times$ & $\times$ & $\times$ & $\times$ \\
Simulated SVT parameterizations & $\times$ & $\times$ & $\times$ & $\times$ \\
Mistag rate & $\times$ & $\times$ & $\times$ & $\times$ \\
$W+$ jets flavor fractions & $\times$ & & $\times$ & \\
\hline \hline
\end{tabular}

Table 9.2: Summary of the systematic uncertainties and how they are correlated. For each systematic uncertainty, the crosses indicates which of $N^{b k g}$ and $\varepsilon_{t o t}^{t \bar{t}}$ is affected, and in which channel. If a systematic uncertainty affects more than one category, the uncertainty is fully correlated between the crossed categories. All systematic uncertainties are fully correlated between the single tagged and the double tagged samples, and between the third and fourth jet multiplicity bins. 
$\varepsilon_{\text {lept }}$ and $\varepsilon_{\text {instr }}$

The largest contribution to the uncertainty on $\varepsilon_{l e p t}$ is the available statistics in the $Z \rightarrow \ell \ell$ sample where it is measured. The statistcal uncertainty on $\varepsilon_{\text {instr }}$ is small, but large systematic uncertainties are assigned to it. The value of $\varepsilon_{\text {instr }}$ is measured in a sample with low $\mathbb{E}_{T}$. The multijet events present in the low $E_{T}$ sample can have different jet flavor content or kinematic properties compared to the multijet events which pass the preselection criteria. The uncertainty on $\varepsilon_{\text {instr }}$ is in large part due to the difficulty of extracting the same type of multijet events present in the signal samples. The relative uncertainty for $\varepsilon_{\text {instr }}$ is much larger than for $\varepsilon_{\text {lept }}$, despite the larger data sample used to measure $\varepsilon_{i n s t r}$. The uncertainties on $\varepsilon_{\text {lept }}$ and $\varepsilon_{\text {instr }}$ are given in Tab. 6.4.

\section{Data-to-Simulation Correction Factors}

The preselection efficiencies for the $t \bar{t}$ signal and the various backgrounds are evaluated in simulated events. If the description of the detector in the simulation is not ideal the efficiency for passing one of the preselection criteria can be biased. To correct for this effect, various data-to-simulation correction factors ${ }^{1}$ are applied to arrive at the preselection efficiencies listed in Tab. 6.5-6.8. The correction factors are uncorrelated between the $e+$ jets and the $\mu+$ jets channels, but fully correlated within each of the two channels. The total systematic uncertainty on the preselection efficiencies from data-to-simulation corrections is approximately $4 \%$ in the $e+$ jets channel and $4.5 \%$ in the $\mu+$ jets channel.

\section{Trigger Efficiencies}

The uncertainty on the efficiency to pass the signal trigger results in systematic uncertainties on the preselection efficiency and tagging probabilities for the $t \bar{t}$ signal and all backgrounds. The signal triggers in the $e+$ jets channel and in the $\mu+$ jets channel both include jet requirements, as was described in Sec. 6.1. The uncertainty on the efficiency for the jet trigger requirements is correlated between all channels. The uncertainty on the trigger requirements for the leptons are only correlated within each of the $e+$ jets and the $\mu+$ jets channels.

\section{Jet Energy Scale}

The jet energy scale corrects the calorimeter-level jet energies to the particle-level energies. Separate jet energy scale corrections are applied to jets in data and jets in Monte Carlo simulation. The effect of the jet energy scale uncertainty on the preselection efficiencies and tagging probabilities is obtained by varying the jet energy

\footnotetext{
${ }^{1} \mathrm{~A}$ data-to-simulation correction factor is derived from the ratio of the efficiency in data to the efficiency in simulation. It brings the efficiency in the simulation to the measured efficiency in data.
} 
scale by $\pm 1 \sigma$, where

$$
\sigma=\sqrt{\sigma_{\text {stat }, \text { data }}^{2}+\sigma_{\text {syst }, \text { data }}^{2}+\sigma_{\text {stat }, \text { sim }}^{2}+\sigma_{\text {syst }, \text { sim }}^{2}} .
$$

Adding the uncertainties from the jet energy scale in data $\left(\sigma_{\text {stat,data }}, \sigma_{\text {syst,data }}\right)$ and the uncertainties from the jet energy scale in the Monte Carlo simulation $\left(\sigma_{\text {stat }, \text { sim }}\right.$, $\left.\sigma_{\text {syst,sim }}\right)$ in quadrature is a conservative approach where the two scales are treated as completely uncorrelated.

\section{Jet Energy Resolution}

The $p_{T}$ spectrum for jets in the Monte Carlo simulation is smeared to match the observed $p_{T}$ resolution of jets in data. The limited knowledge of the parameters for the jet smearing introduces an uncertainty which is not taken into account in the jet energy scale. The uncertainty on the jet energy resolution results in systematic uncertainties on the preselection efficiencies and tagging probabilities for the $t \bar{t}$ signal and all backgrounds.

\section{Jet Reconstruction and Identification Efficiency}

The efficiency for reconstructing a jet which satisfies all the quality selections given in Sec. 4.4 is slightly higher in the Monte Carlo simulation than in data [83]. The discrepancy is limited to low $p_{T}$ jets, typically between $15-25 \mathrm{GeV} / c$. To correct for this effect, a $p_{T}$-dependent data-to-simulation correction factor is determined. Jets are randomly removed in the Monte Carlo simulation according to this correction factor. The uncertainty on the correction factor introduces an uncertainty on the evaluated preselection efficiencies and tagging probabilities.

\section{Taggability Parameterizations}

The taggability parameterizations are derived as functions of jet $p_{T}$ and jet $y$ in the preselected signal samples, as was discussed in Sec. 7.2. The limited statistics in the preselected samples introduces an uncertainty on the taggability parameterization. The difference between the parameterizations derived in the signal samples and the parameterization derived in the multijet sample is treated as a systematic uncertainty on the taggability, as was discussed in Sec. 7.2. Additional sources of systematic uncertainty on the taggability comes from the uncertainties on the heavy-flavor corrections introduced in Sec. 7.2.1.

\section{$b$-tagging Efficiency Measured in Data}

The $b$-tagging efficiency in data is measured in the muon-in-jet sample and is parametrized as a function of jet $p_{T}$ and jet $y$. The limited statistics in the muonin-jet sample introduces an uncertainty on the $b$-tagging efficiency. The $b$-tagging 
efficiency is derived using a system of eight equations, as was described in Sec. 7.3. This method relies on the assumptions that the secondary vertex tagger and the muon tagger are uncorrelated, and that the tagging efficiencies are uncorrelated between the jet containing the muon and the opposite jet. Both assumptions are assigned a systematic uncertainty, derived from a simulated sample of $Z \rightarrow b \bar{b}$ events where one of the $b$ quarks decays semileptonically. The total systematic uncertainty on the $b$-tagging efficiency measured in data is approximately $5 \%$.

\section{Simulated SVT Efficiency Parameterizations}

The $b$-tagging efficiency in data can only be measured for semileptonically decaying $b$ quarks. To obtain the inclusive $b$-tagging efficiency and the efficiency for $c$ quarks, three correction factors are introduced in Eqs. 7.5, 7.6 and 7.8. These correction factors are derived from the efficiency of the secondary vertex algorithm in simulated events.

The tagging efficiency is parameterized as a function of jet $p_{T}$ and jet $y$. The limited statistics available in the simulated samples leads to an uncertainty on the parameterizations. The uncertainty on the parameterizations results in a systematic uncertainty on the predicted tagging probabilities for the $t \bar{t}$ signal and the backgrounds.

\section{Mistag Rate}

The mistag rate is obtained as the product of the negative tag rate, measured in the multijet data sample, and the correction factors $S F_{h f}$ and $S F_{l l}$ introduced in Sec. 7.4.1. The statistical uncertainty on the negative tag rate is small, due to the large statistics available in the multijet data sample where it is measured. A significantly larger uncertainty of $8 \%$ is assigned to the negative tag rate due variations observed when different samples are used to determine the parametrization.

Both $S F_{h f}$ and $S F_{l l}$ are assigned systematic uncertainties from the limited statistics in the simulated multijet sample where they are evaluated. These uncertainties are small compared to the uncertainty on the negative tag rate.

\section{$W+$ jets Flavor Fractions}

The dominant systematic uncertainty assigned to the $W+$ jets flavor fractions come from the comparison with the low statistics MLM-matched samples, as was discussed in Sec. 5.2.2. Excluding the first jet multiplicity bin, the largest discrepancy observed is $\sim 20 \%$. The full $20 \%$ difference is assigned as a systematic uncertainty on all flavor fractions.

There are several smaller systematic uncertainties considered. The effect of varying the requirements for the matching between the partons and the reconstructed jets has been studied. The uncertainty assigned from the choice of matching re- 
quirements is $2 \%$ for the $W c$ fractions and $5 \%$ for the $W b \bar{b}, W(b \bar{b}), W c \bar{c}$ and $W(c \bar{c})$ fractions in all jet multiplicity bins. Uncertainties are also assigned for the choice of parton density functions and for the choice of factorization scale.

The measured heavy-flavor fractions are scaled to account for the difference between the NLO and LO theoretical predictions. The scale factor used is $K=1.05 \pm 0.07$, where the uncertainty comes from the factorization scale and the parton density functions used in the calculation [78].

\subsubsection{Result}

The simplest form of a $t \bar{t}$ cross section likelihood function maximizes the Poisson probability to observe $N$ events when expecting $\tilde{N}$ events,

$$
\mathscr{L}=\frac{\tilde{N}^{N}}{N !} e^{-\tilde{N}} .
$$

The expected number of events $\tilde{N}$ in Eq. 9.4 is given by

$$
\tilde{N}=\sigma_{t \bar{t}} \cdot B(t \bar{t} \rightarrow \ell+\mathrm{jets}) \cdot L \cdot \varepsilon_{t o t}^{t \bar{t}}+N^{b k g} .
$$

where $\sigma_{t \bar{t}}$ is the unknown $t \bar{t}$ cross section, $B(t \bar{t} \rightarrow \ell+$ jets $)$ the branching fraction, $L$ the integrated luminosity, $\varepsilon_{t o t}^{t \bar{t}}$ the total efficiency and $N^{b k g}$ the expected number of background events. The most likely value of $\sigma_{t \bar{t}}$ is obtained from the maximum of $\mathscr{L}$ in Eq. 9.4. When combining several independent channels, the $t \bar{t}$ cross section is obtained from the maximum of the product $\mathscr{L}$ of the individual likelihood functions $\mathscr{L}_{i}$

$$
\mathscr{L}=\prod_{i} \mathscr{L}_{i}
$$

where each $\mathscr{L}_{i}$ is given by Eq. 9.4. In the $t \bar{t}$ cross section measurement in Paper I there are eight independent channels, as was shown in Tab. 9.1. The likelihood function in Eq. 9.6 is referred to as the simple likelihood function.

The likelihood function given in Eq. 9.6 does not allow the systematic uncertainties to influence the value of the measured cross section. Systematic uncertainties on the background or signal predictions are propagated to the measured cross section by varying one systematic source at a time by plus or minus one standard deviation. For every variation, the maximum of the new likelihood function is determined. The difference in cross section with respect to the central value is an estimate of the systematic uncertainty on $\sigma_{t \bar{t}}$ from the varied systematic uncertainty.

The treatment of systematic uncertainties described above can be improved by extending the likelihood function to incorporate all systematic uncertainties. In the new likelihood function, the systematic uncertainties are allowed to change the central value of the cross section. This likelihood function is referred to as the nuisance parameter [86] likelihood function. 
All systematic uncertainties $S_{j}$ are assumed to follow Gaussian distributions with widths $\sigma_{S_{j}}$. Each independent source of systematic uncertainty is modeled in the likelihood function by a free parameter, called a nuisance parameter $\nu_{j}$. The uncertainties $S_{j}$ are expressed as

$$
S_{j}=\nu_{j} \cdot \sigma_{S_{j}}
$$

where $\nu_{j}$ follows a Gaussian probability distribution centered on zero and with a width of one. Each efficiency $\varepsilon$ in the likelihood, for example the $t \bar{t}$ preselection efficiency, is affected by a set of systematic uncertainties. The efficiency $\varepsilon$ is functionally dependent on the corresponding set of nuisance parameters:

$$
\varepsilon=\varepsilon_{\text {central }}+\nu_{1} \sigma_{S_{1}}+\nu_{2} \sigma_{S_{2}}+\ldots
$$

The tagging probabilities, $W+$ jets flavor fractions and efficiencies entering the Matrix Method are also modified accordingly.

Introducing nuisance parameters takes correlations into account in a natural way, by letting the same nuisance parameter affect different efficiencies or tagging probabilities.

The nuisance parameter likelihood function $\mathscr{L}$ used to derive the cross section can be written,

$$
\mathscr{L}=\prod_{i} \mathscr{L}_{i} \prod_{j} \mathscr{G}_{j}
$$

where $\mathscr{L}_{i}$ is given by Eq. 9.4 and $\mathscr{G}_{j}$ is the Gaussian probability distribution for the nuisance parameter $\nu_{j}$. The simple likelihood function in Eq. 9.6 depends only on the cross section $\sigma_{t \bar{t}}$. The likelihood function in Eq. 9.9 is a multi-dimensional function which depends on the cross section $\sigma_{t \bar{t}}$ and the approximately 200 nuisance parameters which models all independent uncertainties. If all the nuisance parameters are fixed to be 0, the likelihood function in Eq. 9.9 reduces to the simple one in Eq. 9.6.

Using the nuisance parameter likelihood function, the measured $t \bar{t}$ production cross section in the $\ell+$ jets final state at $\sqrt{s}=1.96 \mathrm{TeV}$ is:

$$
\sigma_{t \bar{t}}=8.58_{-1.47}^{+1.64}(\text { stat }+ \text { syst }) \pm 0.55 \text { (lumi) pb. }
$$

Table 9.3 summarizes the effect of each systematic uncertainty on the cross section. The shift in the central value of the cross section is given in the column labeled "Offset". The last two columns show the contribution to the positive and negative uncertainties on the cross section. The cross section given in Eq. 9.10 assumes a top quark mass of $175 \mathrm{GeV} / c^{2}$. The dependence of the cross section on the top quark mass is shown in Fig. 9.3. A linear fit in the mass region $160-190 \mathrm{GeV} / c^{2}$ indicates that $\sigma_{t \bar{t}}$ varies by approximately $0.04 \mathrm{pb}$ per $\mathrm{GeV} / c^{2}$. 


\begin{tabular}{|c|c|c|c|c|}
\hline Systematic source & $\sigma_{t \bar{t}}$ & Offset & $\sigma^{+}$ & $\sigma^{-}$ \\
\hline Statistical uncertainty only & 8.48 & - & +1.14 & -1.08 \\
\hline Preselection efficiencies, $\mu+$ jets & & +0.02 & +0.18 & -0.15 \\
\hline Preselection efficiencies, $e+$ jets & & -0.02 & +0.18 & -0.14 \\
\hline Electron trigger requirements & & $<0.01$ & +0.04 & -0.03 \\
\hline Muon trigger requirements & & +0.09 & +0.38 & -0.29 \\
\hline Jet trigger requirements & & $<0.01$ & +0.02 & -0.00 \\
\hline Jet energy scale & & -0.07 & +0.53 & -0.37 \\
\hline Jet energy resolution & & $<0.01$ & +0.02 & -0.02 \\
\hline Jet reconstruction and identification & & -0.07 & +0.33 & -0.20 \\
\hline Taggability & & $<0.01$ & +0.03 & -0.02 \\
\hline Simulated SVT parameterizations & & +0.01 & +0.18 & -0.17 \\
\hline$b$-tagging efficiency in data & & +0.11 & +0.52 & -0.43 \\
\hline Mistag rate & & $<0.01$ & +0.03 & -0.10 \\
\hline$\varepsilon_{\text {lept }}$ and $\varepsilon_{\text {instr }}$ in $e+$ jets channel & & -0.01 & +0.16 & -0.00 \\
\hline$\varepsilon_{l e p t}$ and $\varepsilon_{i n s t r}$ in $\mu+$ jets channel & & -0.01 & +0.12 & -0.01 \\
\hline Event statistics for matrix method & & -0.06 & +0.24 & -0.24 \\
\hline Limited statistics in simulated samples & & $<0.01$ & +0.07 & -0.06 \\
\hline$W+$ jets flavor fractions, from MLM comparison & & +0.04 & +0.45 & -0.45 \\
\hline Remaining unc. on $W+$ jets flavor fractions & & -0.01 & +0.20 & -0.22 \\
\hline Total systematic uncertainty & & - & +1.12 & -0.91 \\
\hline Total unc. (nuisance parameter likelihood) & 8.58 & +0.10 & +1.64 & -1.47 \\
\hline
\end{tabular}

Table 9.3: Summary of the uncertainties on the $t \bar{t}$ cross section in Eq. 9.10, obtained using the nuisance parameter likelihood. This likelihood allows systematic uncertainties to shift the central value of the $t \bar{t}$ cross section. The shift is shown in the column labeled "Offset". The columns labeled $\sigma^{+}$and $\sigma^{-}$show the contributions to the total uncertainty on the cross section.

As a cross check, the $t \bar{t}$ cross section is also derived using the simple likelihood function given in Eq. 9.6. The result,

$$
\sigma_{t \bar{t}}=8.48_{-1.08}^{+1.14} \text { (stat) }{ }_{-0.99}^{+1.21} \text { (syst) } \pm 0.55 \text { (lumi) pb, }
$$

is in good agreement with the result obtained with the nuisance parameter likelihood function. The contribution from each source of systematic uncertainty is shown in Tab. 9.4.

\subsection{Simultaneous Measurement of $\sigma_{t \bar{t}}$ and $R$}

The event tagging probabilities for $t \bar{t}$ events, given in Sec. 8.1.4, are derived under the assumption that the branching fraction $B(t \rightarrow W b)$ is $100 \%$, or equivalently that $R$ in Eq. 9.1 is equal to 1. In the standard model, $R$ can be expressed in terms of the 


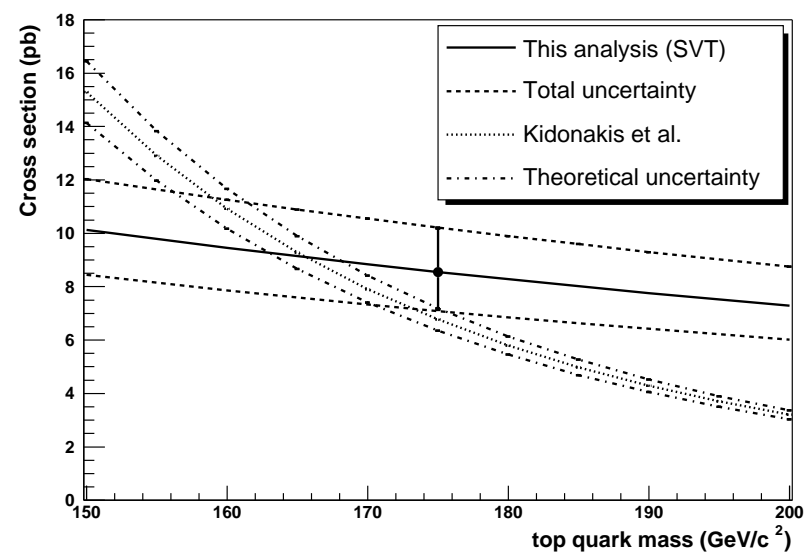

Figure 9.3: Top quark mass dependence of the measured cross section. Also shown is the theoretical prediction for the cross section [28].

\begin{tabular}{|l|cc|}
\hline Systematic source & $\sigma^{+}$ & $\sigma^{-}$ \\
\hline \hline Preselections efficiencies, $e+$ jets & +0.16 & -0.16 \\
Preselections efficiecies, $\mu+$ jets & +0.17 & -0.16 \\
Electron trigger requirements & +0.03 & -0.03 \\
Muon trigger requirements & +0.35 & -0.26 \\
Jet trigger requirements & +0.00 & -0.00 \\
Jet energy scale & +0.65 & -0.43 \\
Jet energy resolution & +0.05 & -0.00 \\
Jet reconstruction and identification & +0.44 & -0.28 \\
Taggability & +0.02 & -0.02 \\
Simulated SVT parameterizations & +0.18 & -0.19 \\
$b$-tagging efficiency in data & +0.52 & -0.47 \\
Mistag rate & +0.07 & -0.07 \\
$\varepsilon_{\text {lept }}$ and $\varepsilon_{\text {instr }}$ in $e+$ jets channel & +0.00 & -0.04 \\
$\varepsilon_{\text {lept }}$ and $\varepsilon_{\text {instr }}$ in $\mu+$ jets channel & +0.01 & -0.02 \\
Event statistics for matrix method & +0.19 & -0.19 \\
Limited statistics in simulated samples & +0.07 & -0.07 \\
$W+$ jets flavor fractions, from MLM comparison & +0.52 & -0.49 \\
Remaining unc. on $W+$ jets flavor fractions & +0.22 & -0.21 \\
\hline Total systematic uncertainty & +1.21 & -0.99 \\
\hline
\end{tabular}

Table 9.4: Summary of the systematic uncertainties on the $t \bar{t}$ cross section in Eq. 9.11, obtained with the simple likelihood function. The cross section derived using the simple likelihood function is treated as a cross check. 
CKM matrix elements as was shown in Eq. 2.8. The assumption that $B(t \rightarrow W b)$ is $100 \%$ is well motivated given the standard model constraint on $V_{t b}$ in Eq. 2.7.

If $R$ is not equal to 1 , the event tagging probabilities for $t \bar{t}$ events are different from those in Tabs. 8.9 and 8.10. The expected fractions of $t \bar{t}$ events with 0,1 and 2 tags will depend on the value of $R$. Using the $b$-tagged signal sample, the standard model prediction of $R \approx 1$ can be tested by treating it as a free parameter in the $t \bar{t}$ cross section likelihood function.

\subsection{1 $R$-dependence of Event Tagging Probabilities for $t \bar{t}$ Events}

If $R$ is less than 1 , the decay of the two top quarks in a $t \bar{t}$ event can produce either 0,1 or $2 b$ quarks. The probabilities to obtain each of the three final states are $(1-R)^{2}, 2 R(1-R)$ and $R^{2}$ respectively. The event tagging probabilities are derived separately for the three possibilities:

1. $t \bar{t} \rightarrow W b W \bar{b}$ (later referred to as $t \bar{t} \rightarrow b \bar{b})$

2. $t \bar{t} \rightarrow W b W \bar{q}_{l}$ or, equivalently, $t \bar{t} \rightarrow W q_{l} W \bar{b}$ (both later referred to as $t \bar{t} \rightarrow b \bar{q}_{l}$ )

3. $t \bar{t} \rightarrow W q_{l} W \bar{q}_{l}$ (later referred to $t \bar{t} \rightarrow q_{l} \bar{q}_{l}$ ),

where $q_{l}$ is a light down-type quark $\left(q_{l}=d\right.$ or $\left.s\right)$. The tagging probabilities for $t \bar{t} \rightarrow b \bar{b}$ events are unchanged, and are given in Tab. 8.9 and Tab. 8.10. The event tagging probabilities for $t \bar{t} \rightarrow b \bar{q}_{l}$ and $t \bar{t} \rightarrow q_{l} \bar{q}_{l}$ events are given in Tab. 9.5 for single tagged events and in Tab. 9.6 for double tagged events.

\begin{tabular}{c|cc}
\hline \hline & 3 jets & $\geq 4$ jets \\
\hline \hline \multicolumn{3}{c}{$e+$ jets channel } \\
\hline \hline$t \bar{t} \rightarrow b \bar{q}_{l}$ & $32.3 \pm 0.3$ & $37.0 \pm 0.2$ \\
$t \bar{t} \rightarrow q_{l} \bar{q}_{l}$ & $5.5 \pm 0.1$ & $7.8 \pm 0.1$ \\
\hline \hline \multicolumn{3}{c}{$\mu+$ jets channel } \\
\hline \hline$t \bar{t} \rightarrow b \bar{q}_{l}$ & $32.2 \pm 0.3$ & $36.3 \pm 0.2$ \\
$t \bar{t} \rightarrow q_{l} \bar{q}_{l}$ & $5.6 \pm 0.1$ & $7.5 \pm 0.1$ \\
\hline \hline
\end{tabular}

Table 9.5: Single tag probabilities (in \%) for $t \bar{t}$ events where the two top quarks decay into one or two light-flavor quarks. Only statistical uncertainties are shown.

The event tagging probabilities in Tabs. 8.9, 8.10, 9.5 and 9.6 can be combined to obtain the $t \bar{t}$ event tagging probability $P(t \bar{t})$ as a function of $R$ :

$$
P(t \bar{t})=R^{2} P(t \bar{t} \rightarrow b \bar{b})+2 R(1-R) P\left(t \bar{t} \rightarrow b \bar{q}_{l}\right)+(1-R)^{2} P\left(t \bar{t} \rightarrow q_{l} \bar{q}_{l}\right) .
$$

The dependence of the event tagging probabilities on $R$ are shown in Fig. 9.4. 


\begin{tabular}{c|cc}
\hline \hline & 3 jets & $\geq 4$ jets \\
\hline \hline \multicolumn{3}{c}{$e+$ jets channel } \\
\hline \hline$t \bar{t} \rightarrow b \bar{q}_{l}$ & $1.36 \pm 0.04$ & $2.26 \pm 0.04$ \\
$t \bar{t} \rightarrow q_{l} \bar{q}_{l}$ & $0.076 \pm 0.007$ & $0.20 \pm 0.01$ \\
\hline \hline \multicolumn{3}{c}{$\mu+$ jets channel } \\
\hline \hline$t \bar{t} \rightarrow b \bar{q}_{l}$ & $1.35 \pm 0.04$ & $2.24 \pm 0.04$ \\
$t \bar{t} \rightarrow q_{l} \bar{q}_{l}$ & $0.077 \pm 0.008$ & $0.17 \pm 0.01$ \\
\hline \hline
\end{tabular}

Table 9.6: Double tag probabilities (in \%) for $t \bar{t}$ events where the two top quarks decay into one or two light-flavor quarks. Only statistical uncertainties are shown.
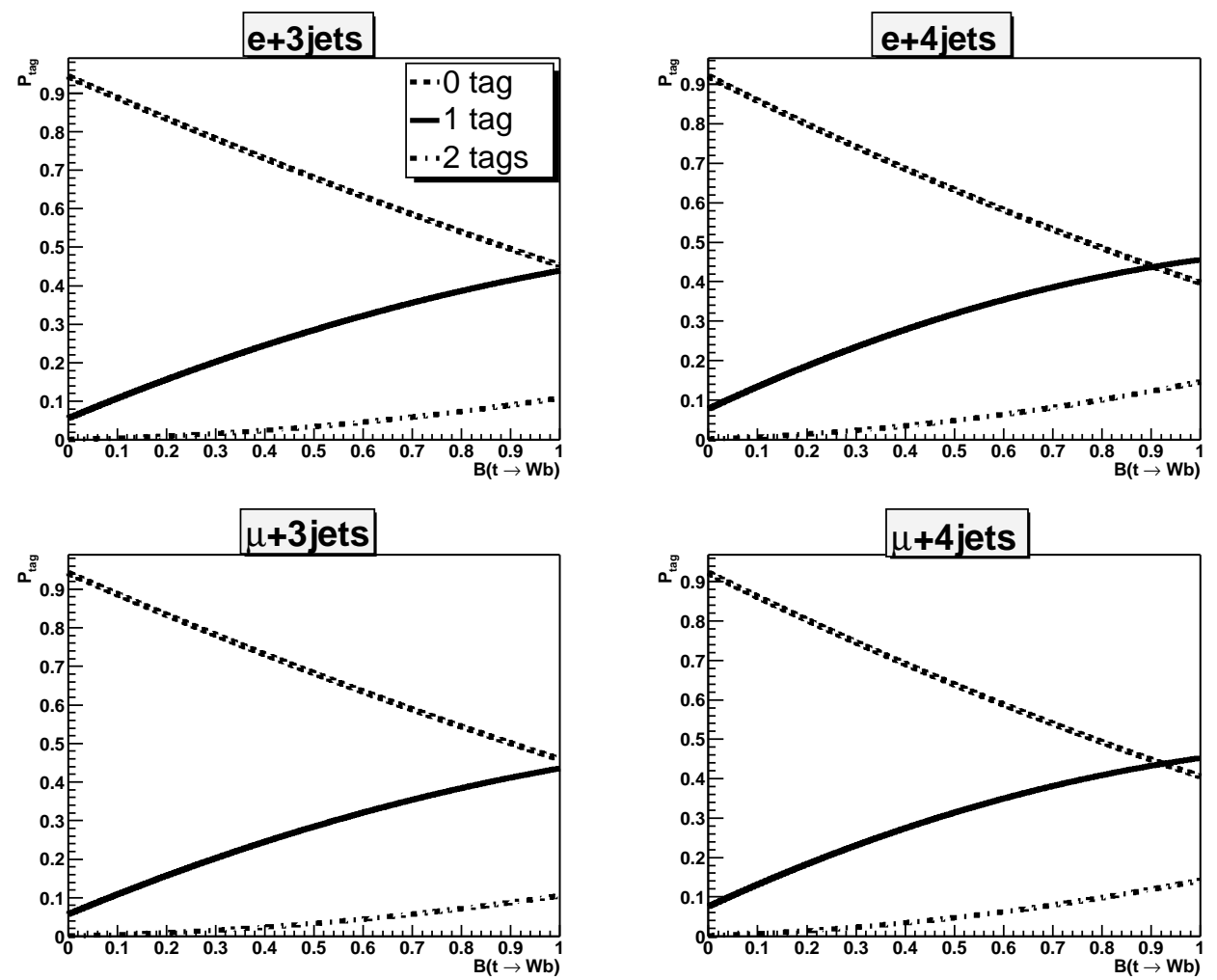

Figure 9.4: Event tagging probability dependence on $R$ for $t \bar{t}$ events. 


\begin{tabular}{l|ccc}
\hline \hline$\ell+\mathbf{3}$ jets & 0 tags & $\mathbf{1 ~ t a g}$ & $\geq 2$ tags \\
\hline$W+$ jets & $1039.9 \pm 37.9$ & $34.1 \pm 4.6$ & $2.4 \pm 0.4$ \\
Multijet & $192.2 \pm 23.1$ & $8.3 \pm 1.5$ & $0.1 \pm 0.3$ \\
Other Backgrounds & $18.4 \pm 1.3$ & $4.3 \pm 0.3$ & $0.7 \pm 0.1$ \\
\hline$t \bar{t} \rightarrow \ell+$ jets & $29.5 \pm 1.6$ & $28.2 \pm 1.6$ & $6.9 \pm 0.5$ \\
\hline Total Expected & $1280 \pm 44$ & $74.9 \pm 5.1$ & $10.1 \pm 0.8$ \\
\hline Observed & 1277 & 79 & 9 \\
\hline \hline$\ell \geq 4$ jets & $\mathbf{0 ~ t a g s}$ & $\mathbf{1 ~ t a g}$ & $\geq 2$ tags \\
\hline$W+$ jets & $203.1 \pm 17.2$ & $9.2 \pm 1.2$ & $0.7 \pm 0.1$ \\
Multijet & $65.4 \pm 8.9$ & $4.1 \pm 1.1$ & $0.0 \pm 0.4$ \\
Other Backgrounds & $2.9 \pm 0.4$ & $1.2 \pm 0.2$ & $0.2 \pm 0.0$ \\
\hline$t \bar{t} \rightarrow \ell+$ jets & $32.5 \pm 3.0$ & $36.3 \pm 3.3$ & $11.4 \pm 1.4$ \\
\hline Total Expected & $304 \pm 20$ & $50.8 \pm 3.7$ & $12.3 \pm 1.4$ \\
\hline Observed & 291 & 62 & 14 \\
\hline \hline
\end{tabular}

Table 9.7: Observed and predicted number of events for $R=1$ and $\sigma_{t \bar{t}}=7 \mathrm{pb}$ in the $\ell+$ jets channel.

The mistag rate for light-quark jets is derived in the multijet data sample, as was described in Sec. 7.4. The multijet sample is dominated by jets from $u$ quarks and $d$ quarks. In the standard model, the top quark branching fraction to $s$ quarks is larger than the branching fraction to $d$ quarks, $B(t \rightarrow W s) / B(t \rightarrow W d) \sim 10$. If the tagging efficiency for $s$-quark jets is higher than for $u$-or $d$-quark jets, the event tagging probabilities in Tab. 9.5 and Tab. 9.6 are underestimated.

The difference in tagging efficiency for $s$-quark jets and $u$ - and $d$-quark jets has been studied in a simulated sample of multijet events and no significant difference is observed.

\subsubsection{Number of $t \bar{t}$ Events in the Sample Without $b$-tags}

The number of expected $t \bar{t}$ events with 0,1 and $2 b$-tagged jets depends on the cross section $\sigma_{t \bar{t}}$ and the ratio $R$. Table. 9.7 summarizes the expected and observed number of events with 0,1 and $2 b$-tags using the standard model predictions of $\sigma_{t \bar{t}}=7 \mathrm{pb}$ and $R=1$. The observed number of $t \bar{t}$ events with 1 and $2 b$-tags can be determined from the excess of single and double tagged events over the background, as discussed in Sec. 9.1. For events without $b$-tags, the expected number of $t \bar{t}$ events is approximately the same as the uncertainty on the background prediction. In order to measure the value of $R$ better separation is needed between the $t \bar{t}$ events and the background events in the sample without $b$-tags.

A topological likelihood discriminant for $t \bar{t}$ events is constructed using kinematic variables which are sensitive to differences between $t \bar{t}$ events and background events. 
Using this topological discriminant, the number of $t \bar{t}$ events in the sample without $b$-tags can be estimated. This is described below.

\section{Topological Likelihood Discriminant for $t \bar{t}$ Events}

The four topological variables which are inputs to the likelihood discriminant are

- sphericity $\mathcal{S}$

- centrality $\mathcal{C}$

- $H_{T 2}^{\prime}$

- $K_{\text {Tmin }}^{\prime}$

which are described in Sec. 8.3. The distributions of the four input variables in the sample without b-tags are shown in Fig. 8.7, Fig. 8.9, Fig. 8.6 and Fig. 8.10 respectively.

To be less sensitive to statistical fluctuations in the distributions of the input variables, the logarithm of the four variables is used. The probability density functions for $t \bar{t}$ events, $P^{t \bar{t}}$, and $W+$ jets events, $P^{W}$, in the transformed variables are shown in Figs. 9.5 and 9.6.

The ratio of the probability density functions for signal $S$ and background $B$ is built for each of the four input variables

$$
S_{i} / B_{i}=\frac{P_{i}^{t \bar{t}}}{P_{i}^{W}} \quad i=1,2,3,4 .
$$

The logarithm of each of the four ratios are fitted with polynomials. The fit, later referred to as $\left(\ln \frac{S}{B}\right)^{f i t}$, reduces the sensitivity to single events. The fits for all four input variables are shown in Figs. 9.7 and 9.8.

The optimal likelihood discriminant $\mathscr{L}_{\text {optimal }}$ for $N$ input variables is

$$
\mathscr{L}_{\text {optimal }}=\frac{S\left(x_{1}, \ldots, x_{N}\right)}{S\left(x_{1}, \ldots, x_{N}\right)+B\left(x_{1}, \ldots, x_{N}\right)}
$$

where $S$ and $B$ are the $N$-dimensional probability density functions for signal and background respectively, and $x_{1}, \ldots, x_{N}$ are the measured values of the input variables. If the input variables are assumed to be uncorrelated, the $N$-dimensional probability density functions are equal to the product of $N$ one-dimensional probability density functions:

$$
\begin{aligned}
& S\left(x_{1}, \ldots, x_{N}\right)=\prod_{i=1}^{N} S_{i}\left(x_{i}\right) \\
& B\left(x_{1}, \ldots, x_{N}\right)=\prod_{i=1}^{N} B_{i}\left(x_{i}\right)
\end{aligned}
$$



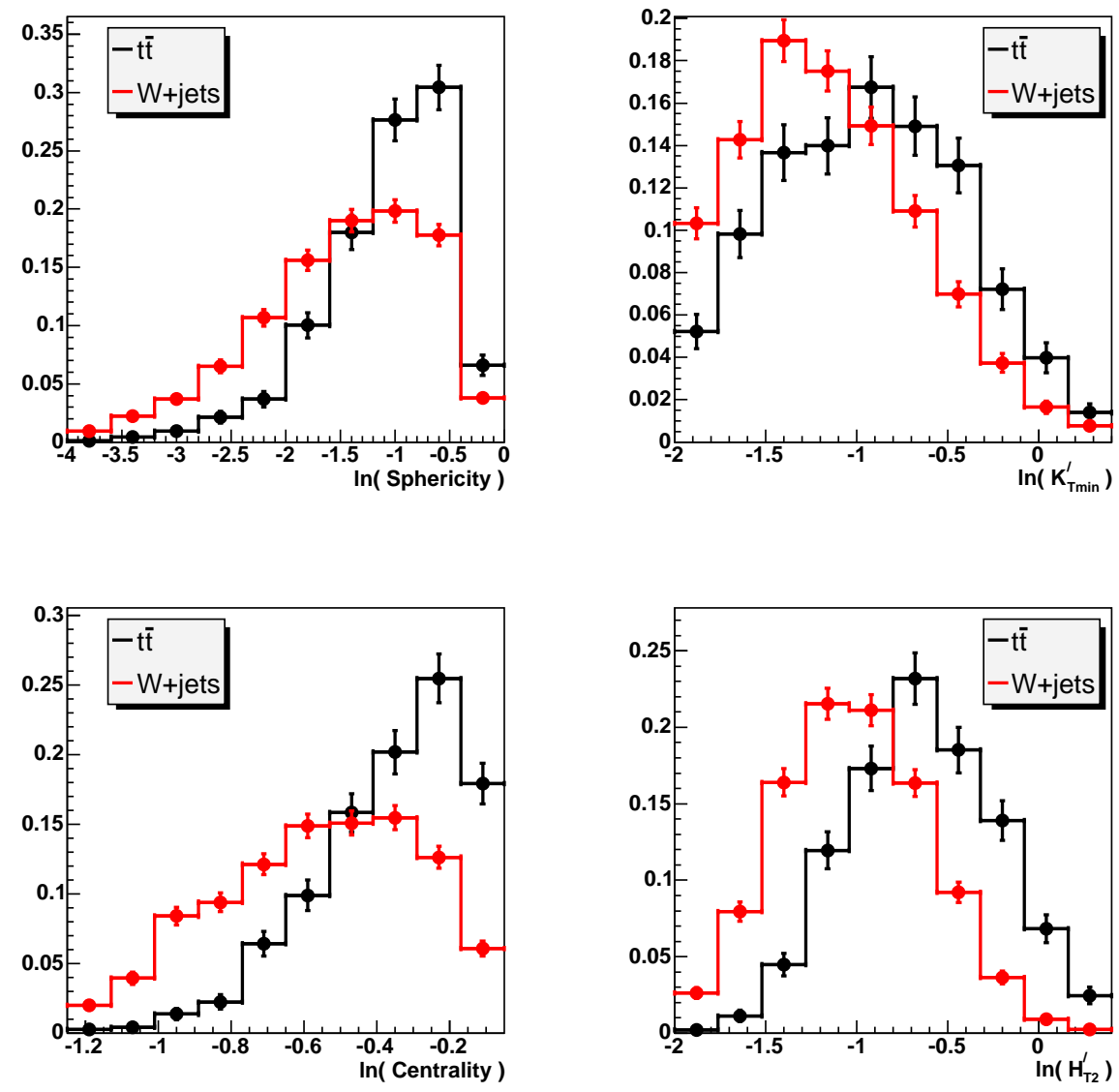

Figure 9.5: Probability density functions of the transformed input variables to the topological likelihood discriminant for $W+$ jets events and $t \bar{t}$ events in the $e+4$ jets channel.

Assuming that the four variables $\mathcal{S}, \mathcal{C}, H_{T 2}^{\prime}$ and $K_{T m i n}^{\prime}$ are uncorrelated, the topological likelihood discriminant for $t \bar{t}$ events $\mathscr{L}_{t \bar{t}}$ can be written as:

$$
\begin{aligned}
\mathscr{L}_{t \bar{t}} & =\frac{\prod_{i=1}^{4} S_{i}\left(x_{i}\right)}{\prod_{i=1}^{4} S_{i}\left(x_{i}\right)+\prod_{i=1}^{4} B_{i}\left(x_{i}\right)}=\frac{\prod_{i=1}^{4} S_{i}\left(x_{i}\right) / B_{i}\left(x_{i}\right)}{\prod_{i=1}^{4} S_{i}\left(x_{i}\right) / B_{i}\left(x_{i}\right)+1} \\
& =\frac{\exp \left(\prod_{i=1}^{4} \ln \frac{S_{i}\left(x_{i}\right)}{B_{i}\left(x_{i}\right)}\right)}{\exp \left(\prod_{i=1}^{4} \ln \frac{S_{i}\left(x_{i}\right)}{B_{i}\left(x_{i}\right)}\right)+1}
\end{aligned}
$$



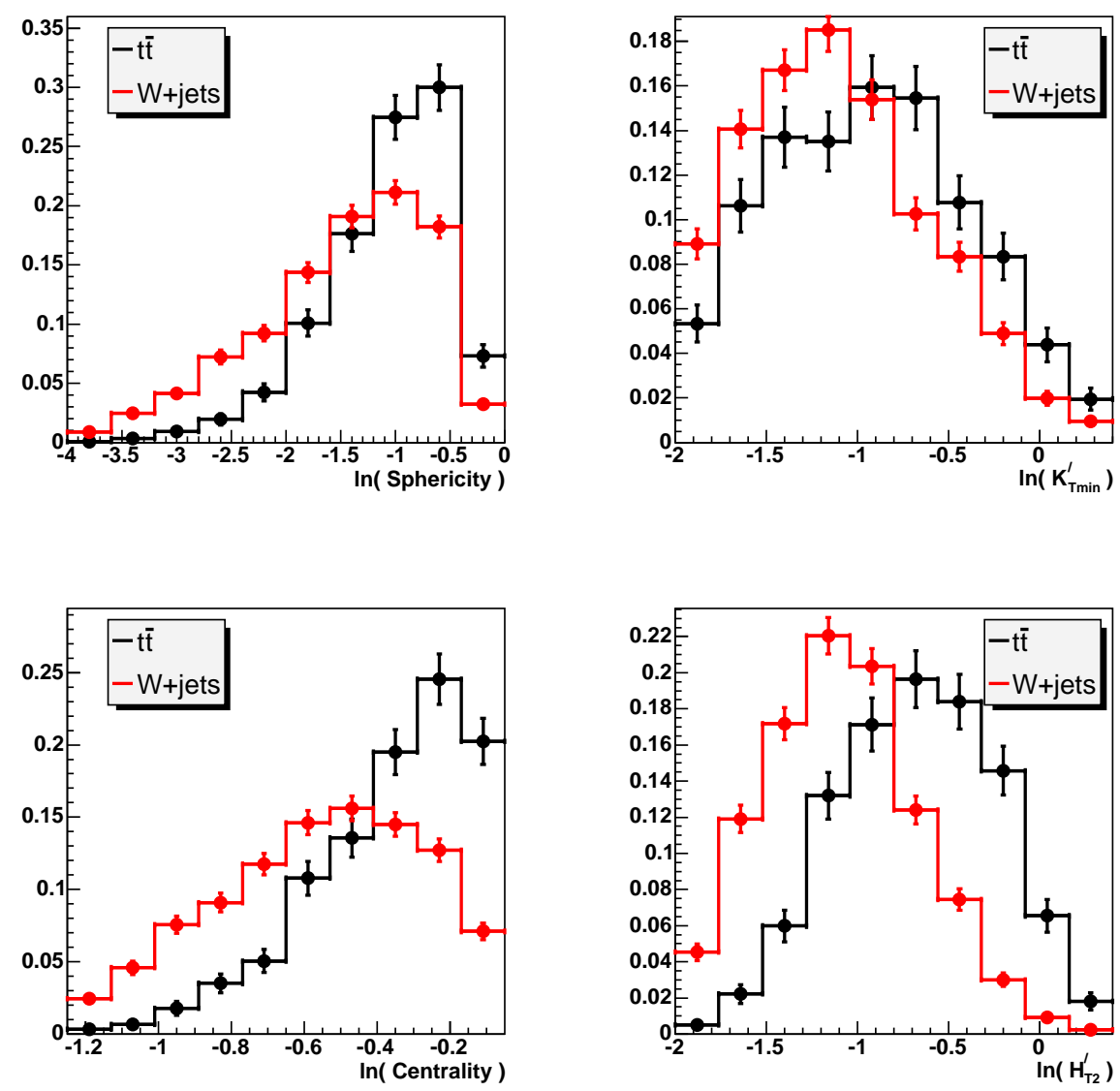

Figure 9.6: Probability density functions of the transformed input variables to the topological likelihood discriminant for $W+$ jets events and $t \bar{t}$ events in the $\mu+4$ jets channel.

Using the fitted functions $\left(\ln \frac{S}{B}\right)_{i}^{f i t}$ in Figs. 9.7 and 9.8 , the likelihood function in Eq. 9.17 can be written:

$$
\mathscr{L}_{t \bar{t}}=\frac{\exp \left(\prod_{i=1}^{4}\left(\ln \frac{S}{B}\right)_{i}^{f i t}\left(x_{i}\right)\right)}{\exp \left(\prod_{i=1}^{4}\left(\ln \frac{S}{B}\right)_{i}^{f i t}\left(x_{i}\right)\right)+1}
$$

This topological likelihood function shows a much better discrimination between $t \bar{t}$ events and background events than any single one of the four input variables. It can therefore be used to estimate the number of $t \bar{t}$ events in the sample without $b$-tags. 

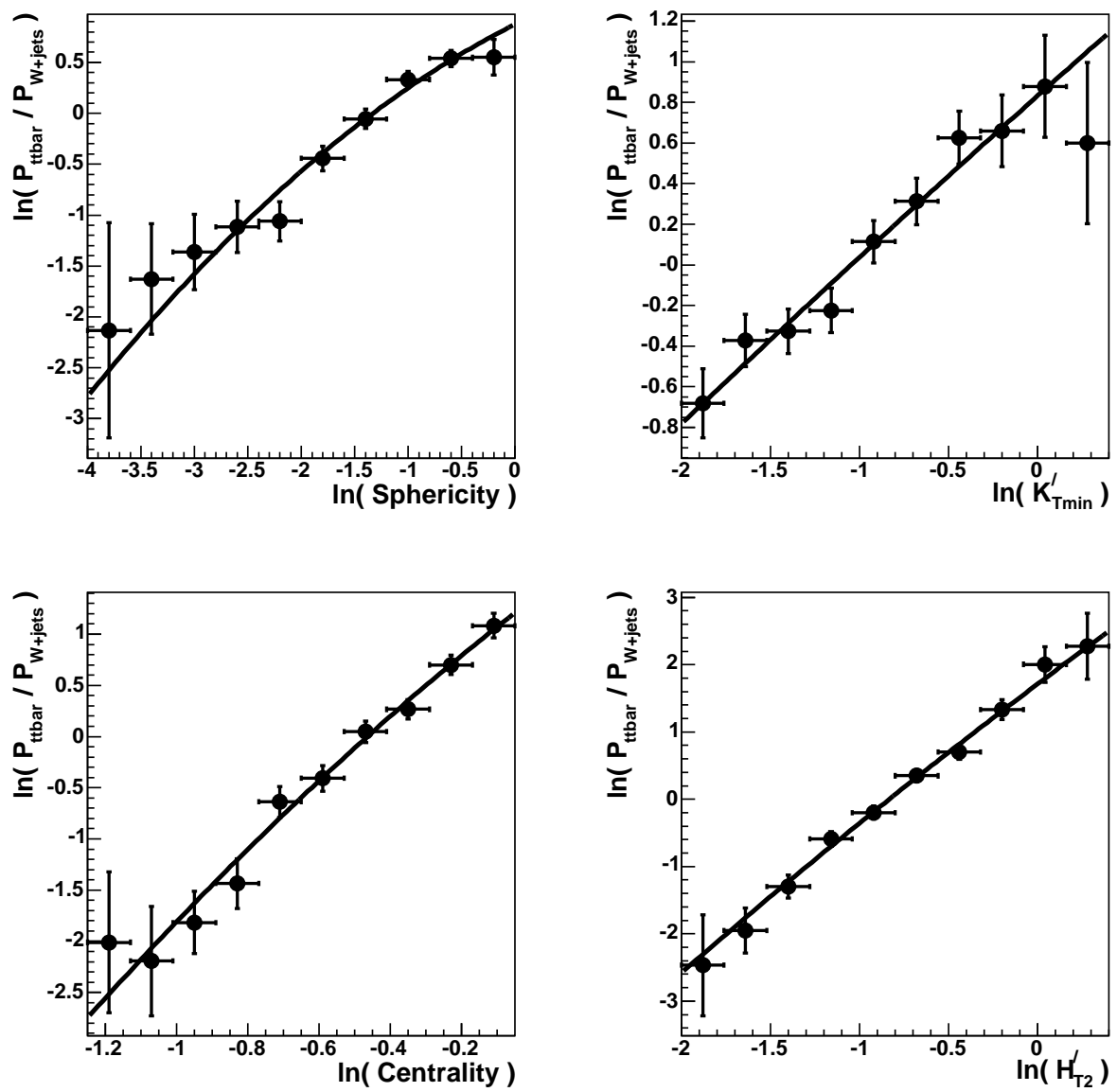

Figure 9.7: Fit to the logarithm of the probability density function for signal over background for each of the transformed input variables to the $t \bar{t}$ likelihood discriminant in the $e+4$ jets channel.

\section{Template Fitting}

The topological likelihood discriminant, defined in Eq. 9.18, is used to build templates for $t \bar{t}$ events, $W+$ jets events and multijet events. These templates are shown in Fig. 9.9. The value of the template in each of the 10 bins shows the fraction of events $f_{i}$ which falls into bin $i$.

The topological likelihood distribution observed in data can be fitted with a sum of the three templates for $t \bar{t}$ events, $W+$ jets events and multijet events. Physics backgrounds other than $W+$ jets are assumed to be modeled by the $W+$ jets template. The best fit maximizes the total probability $\mathscr{L}_{\text {template }}$, defined as

$$
\mathscr{L}_{\text {template }}=\prod_{i=1}^{10} \mathcal{P}\left(n_{i}^{o b s} ; \mu_{i}\right)
$$



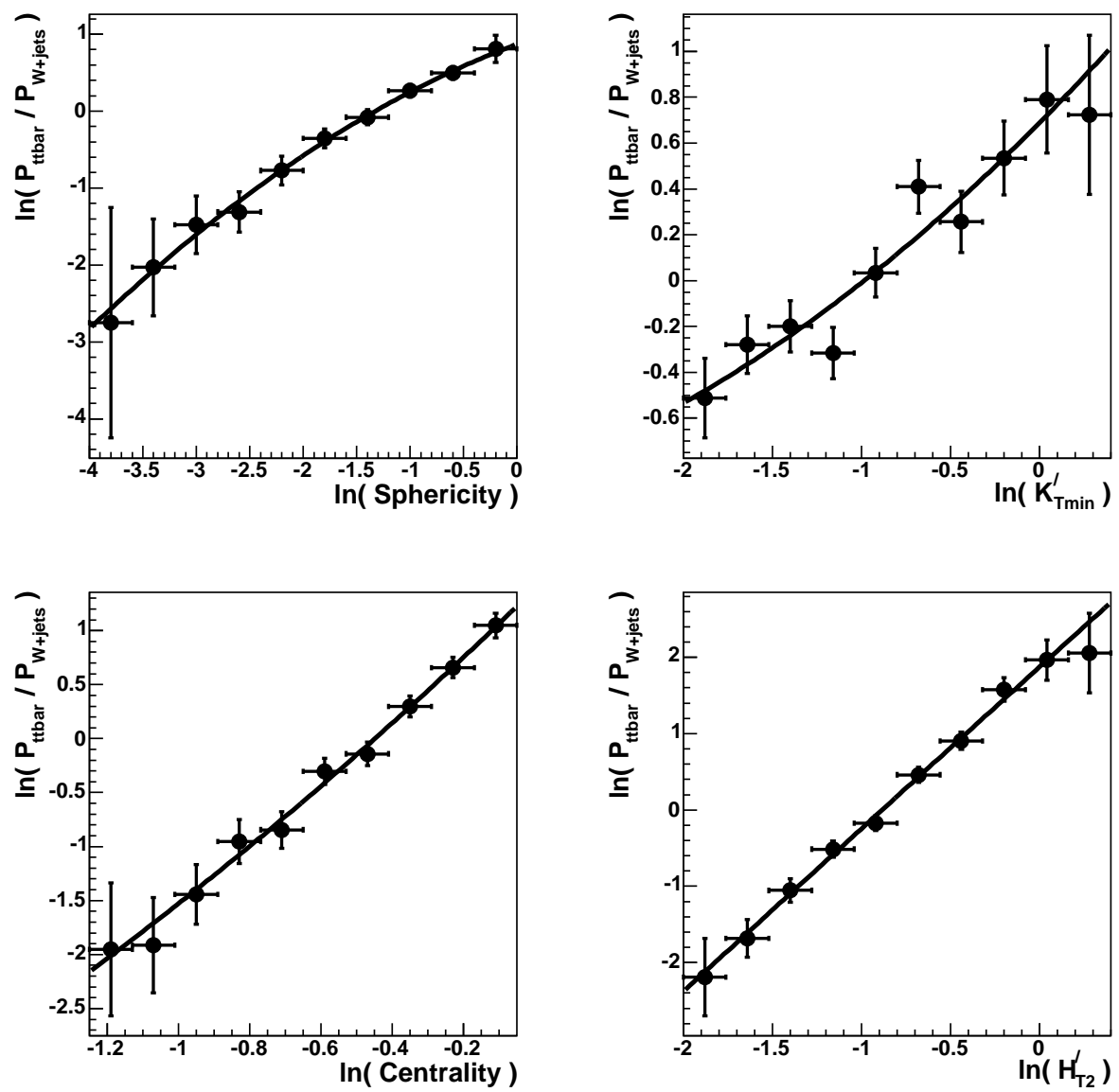

Figure 9.8: Fit to the logarithm of the probability density function for signal over background for each of the transformed input variables to the $t \bar{t}$ likelihood discriminant in the $\mu+4$ jets channel.

which is the product of the Poisson probabilities for each bin $i$ to observe $n_{i}^{\text {obs }}$ events when $\mu_{i}$ events is expected. The expected number of events in each bin $\mu_{i}$ is a function of the number of $t \bar{t}$ events $N^{t \bar{t}}$, the number of $W+$ jets events $N^{W+\text { jets }}$ and the number of multijet events $N^{\text {instr }}$ in the sample:

$$
\mu_{i}\left(N^{t \bar{t}}, N^{W+\text { jets }}, N^{i n s t r}\right)=f_{i}^{t \bar{t}} N^{t \bar{t}}+f_{i}^{W+\text { jets }} N^{W+\text { jets }}+f_{i}^{i n s t r} N^{i n s t r}
$$

The fractions $f_{i}^{t \bar{t}}, f_{i}^{W+\text { jets }}$ and $f_{i}^{i n s t r}$ are given by the templates. To constrain the number of $t \bar{t}$ events without $b$-tags in the fourth jet multiplicity bin, the template likelihood $\mathscr{L}_{\text {template }}$ in Eq. 9.19 is implemented in the nuisance parameter likelihood function for the simultaneous measurement of $\sigma_{t \bar{t}}$ and $R$. In the third jet multiplicity bin the expected number of $t \bar{t}$ events without $b$-tags is too small to be separated from the background. 

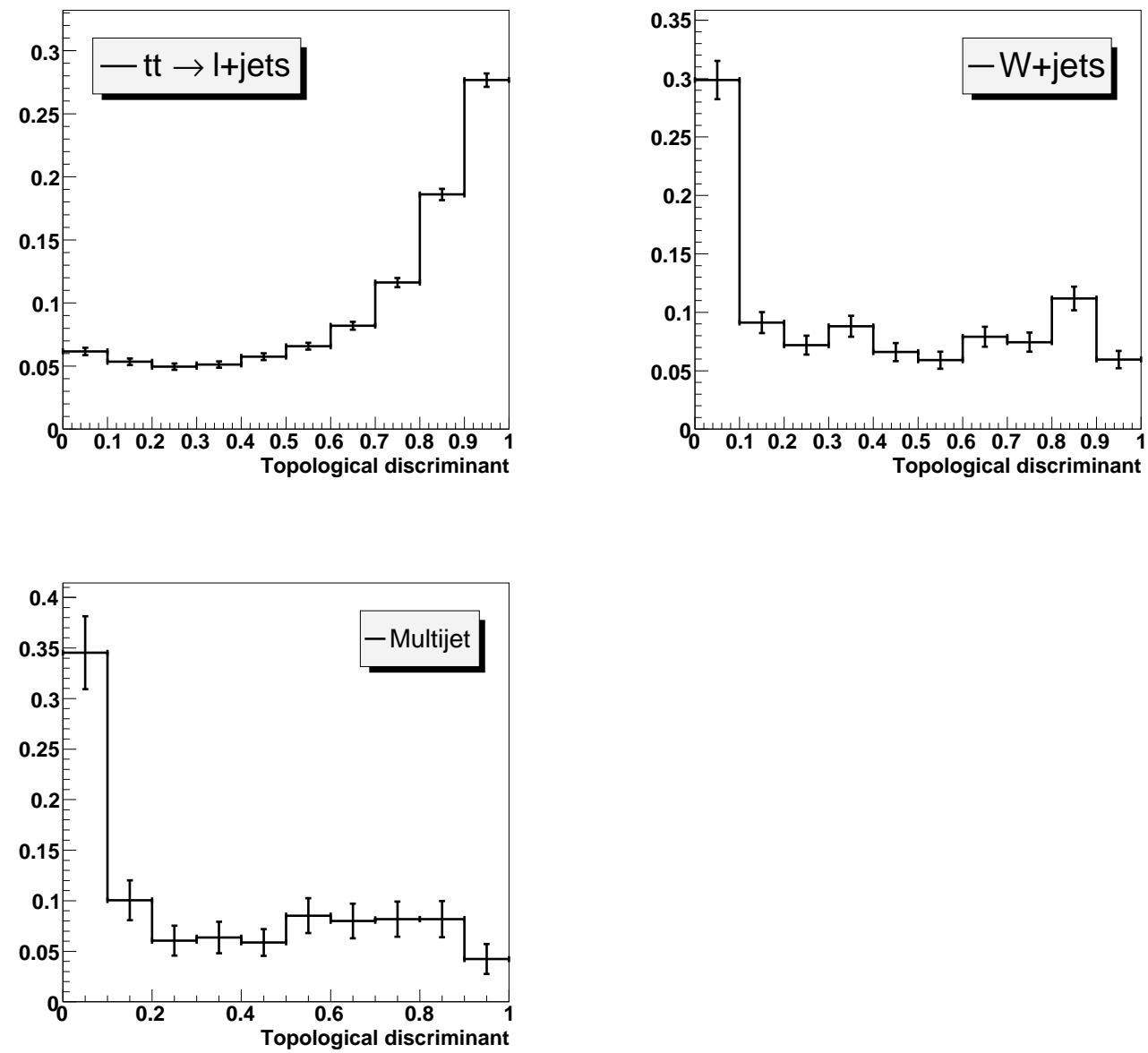

Figure 9.9: Topological likelihood templates in the $\ell+4$ jets channel. The template for the $t \bar{t}$ signal peaks at a likelihood value of 1 , whereas the background $W+$ jets and multijet templates peak at a likelihood value of 0 .

The template likelihood in Eq. 9.19 can also be used to perform a standalone fit to the data. This is done, as a cross check, in the samples with 0,1 and $2 b$-tags. The template fit in the sample without $b$-tags is shown in Fig. 9.10 and it indicates that the number of $t \bar{t}$ events in this sample is small. The template fits in the single and double tagged samples are shown in Fig. 9.11 and Fig. 9.12. The obtained number of $t \bar{t}$ events, $W+$ jets events and multijet events are in good agreement with the predictions from the $t \bar{t}$ cross section measurement in Tab. 8.12 and Tab. 8.14. 


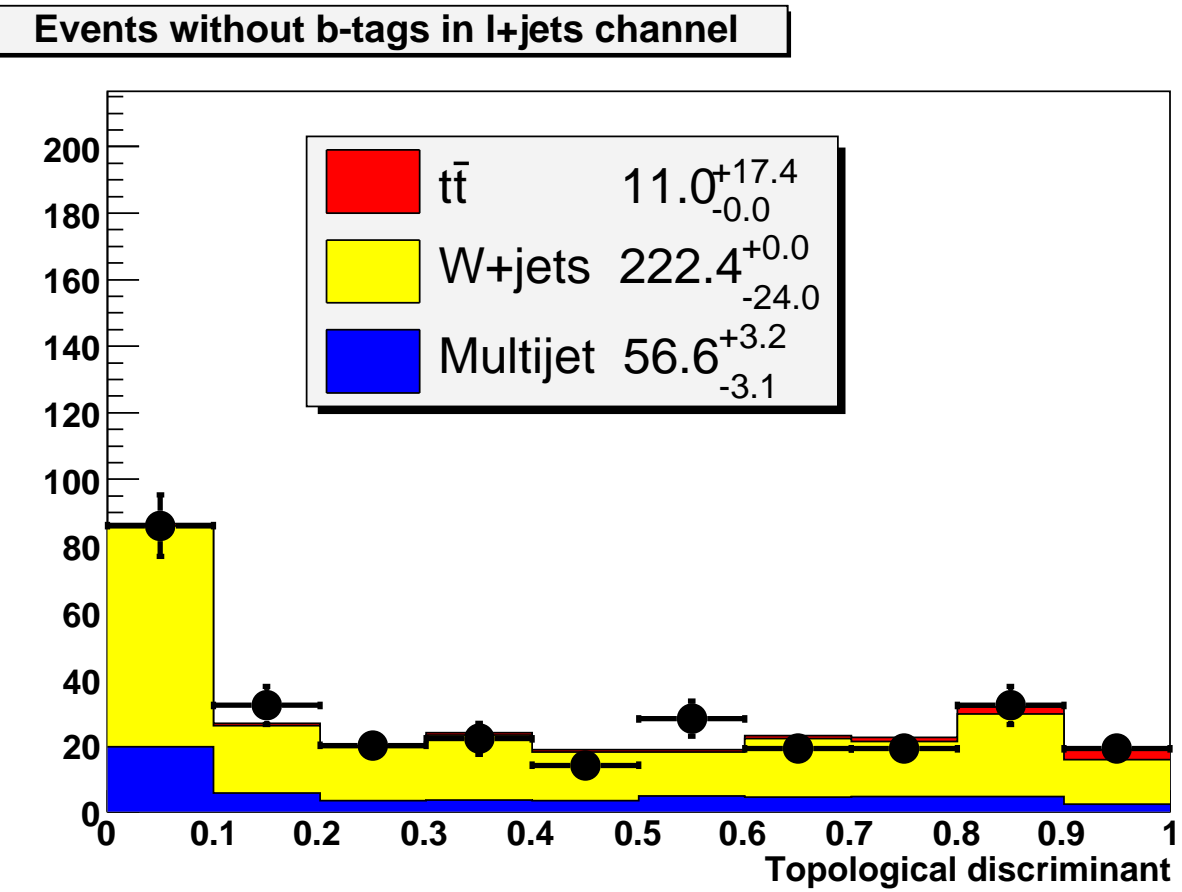

Figure 9.10: Topological likelihood template fit to the $\ell+4$ jets events without $b$-tags. The template fit indicates that there are few $t \bar{t}$ events in this sample. 


\section{Single tagged events in I+jets channel}

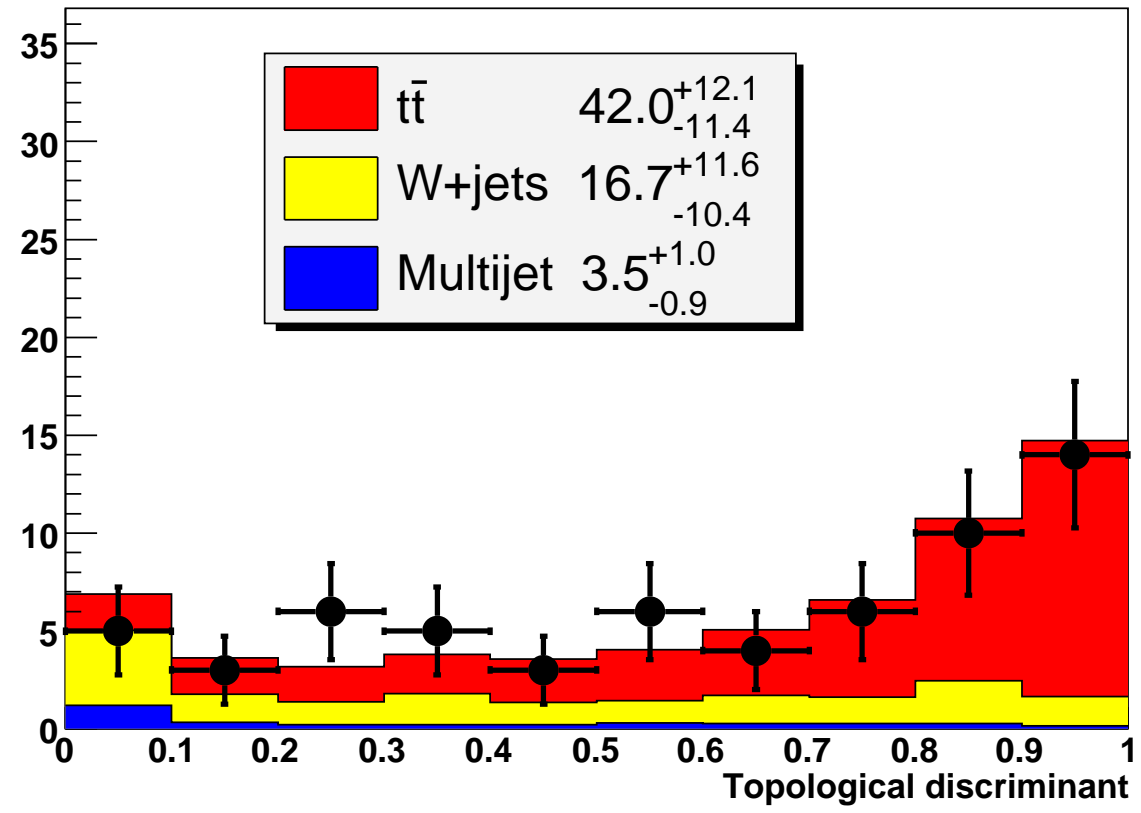

Figure 9.11: Topological likelihood template fit to the single tagged events in the $\ell+4$ jets sample. 


\section{Double tagged events in I+jets channel}

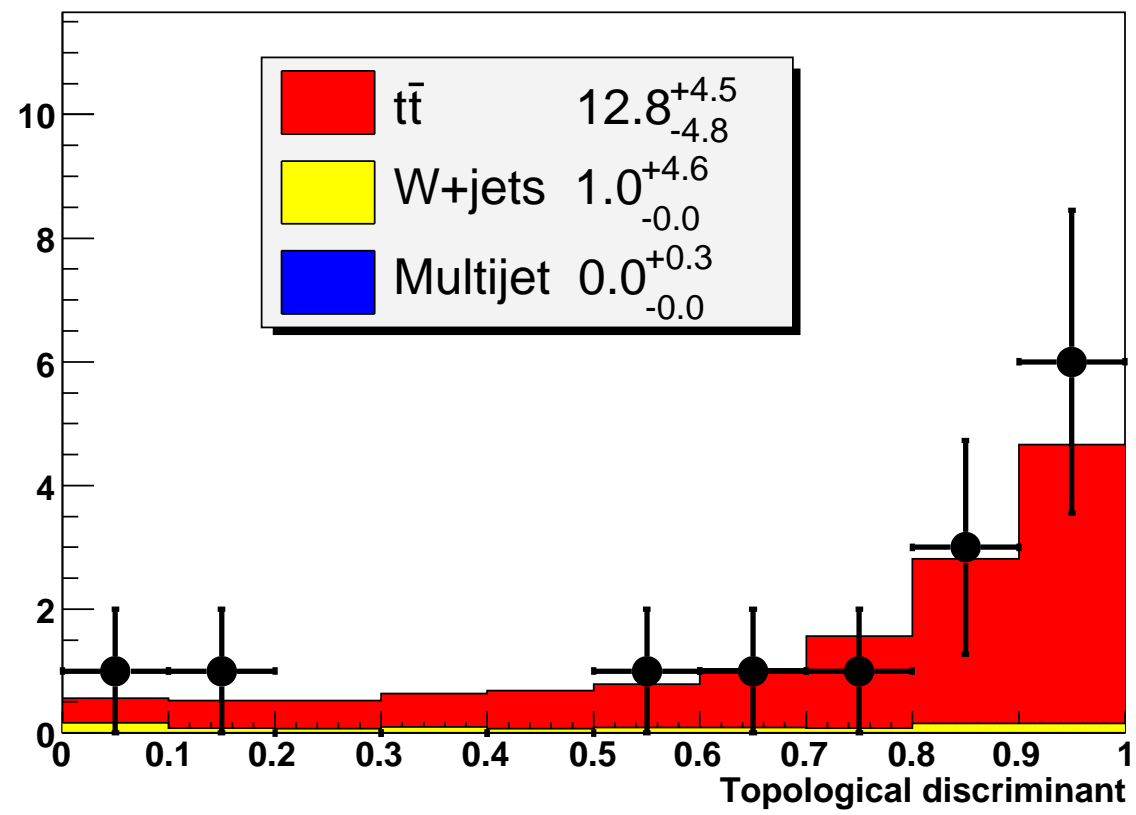

Figure 9.12: Topological likelihood template fit to the double tagged events in the $\ell+4$ jets sample. 


\subsubsection{Systematic Uncertainties}

The sources of systematic uncertainty in the simultaneous measurement of $\sigma_{t \bar{t}}$ and $R$ are identical to the ones in the $t \bar{t}$ cross section measurement, described in Sec. 9.1.1. Some of the sources will affect the topological likelihood templates in Fig. 9.9. The shift in the template shape from the largest systematic uncertainties (jet energy scale, jet energy resolution and jet reconstruction and identification efficiency) is shown in Fig. 9.13 for the $t \bar{t}$ template. The shift in the template shape for the $W+$ jets template is shown in Fig. 9.14. The choice of factorization scale for the generation of the $W+$ jets sample is found to have a significant effect on the template shape for $W+$ jets events.
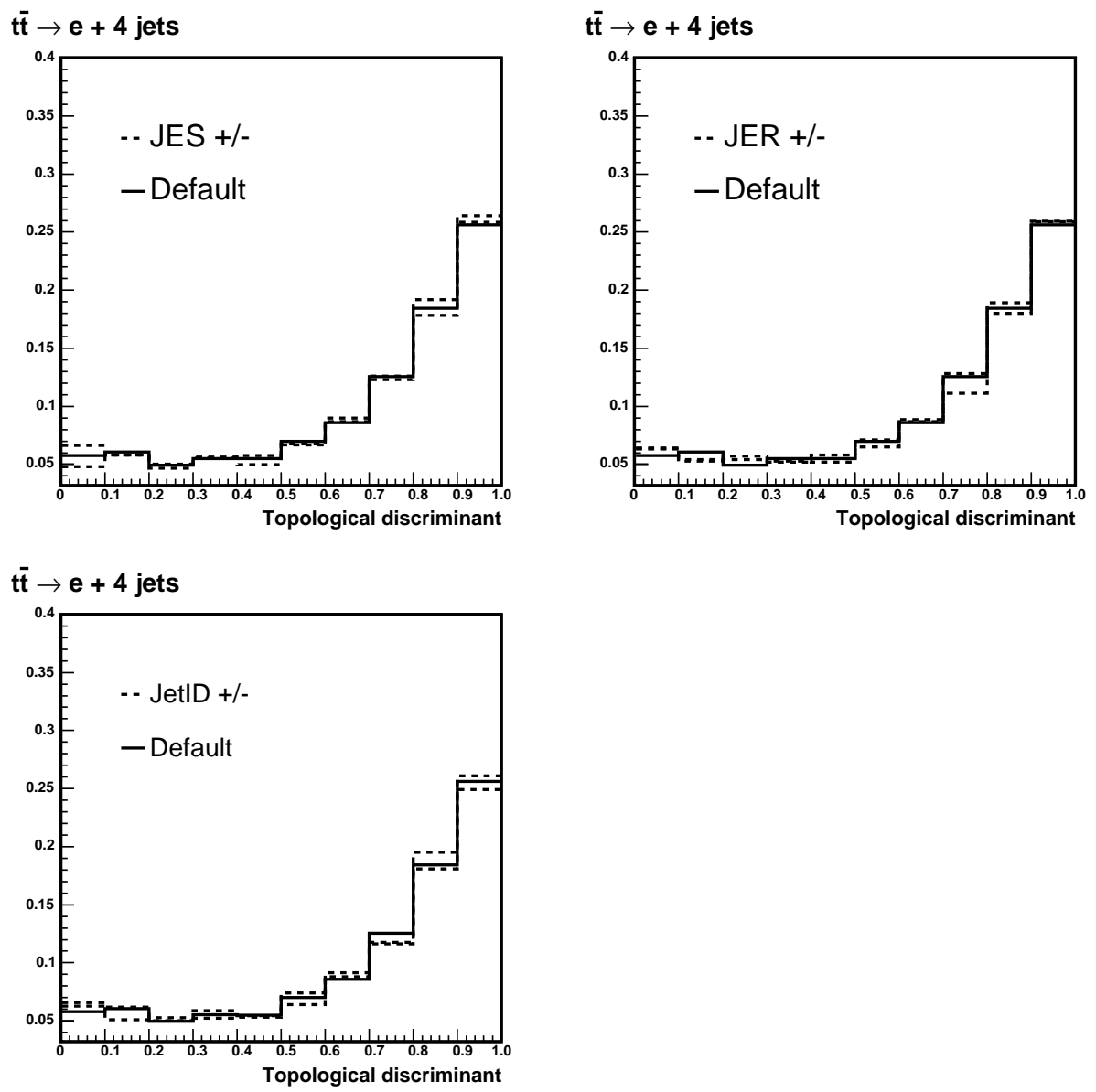

Figure 9.13: Topological likelihood templates in the $e+$ jets channel for $t \bar{t}$. The solid line shows the default template shape, superimposed on the template shapes obtained by varying jet energy scale (JES), jet energy resolution (JER) and jet reconstruction and identification efficiency (JetID). Similar shifts are observed in the $\mu+$ jets channel. 

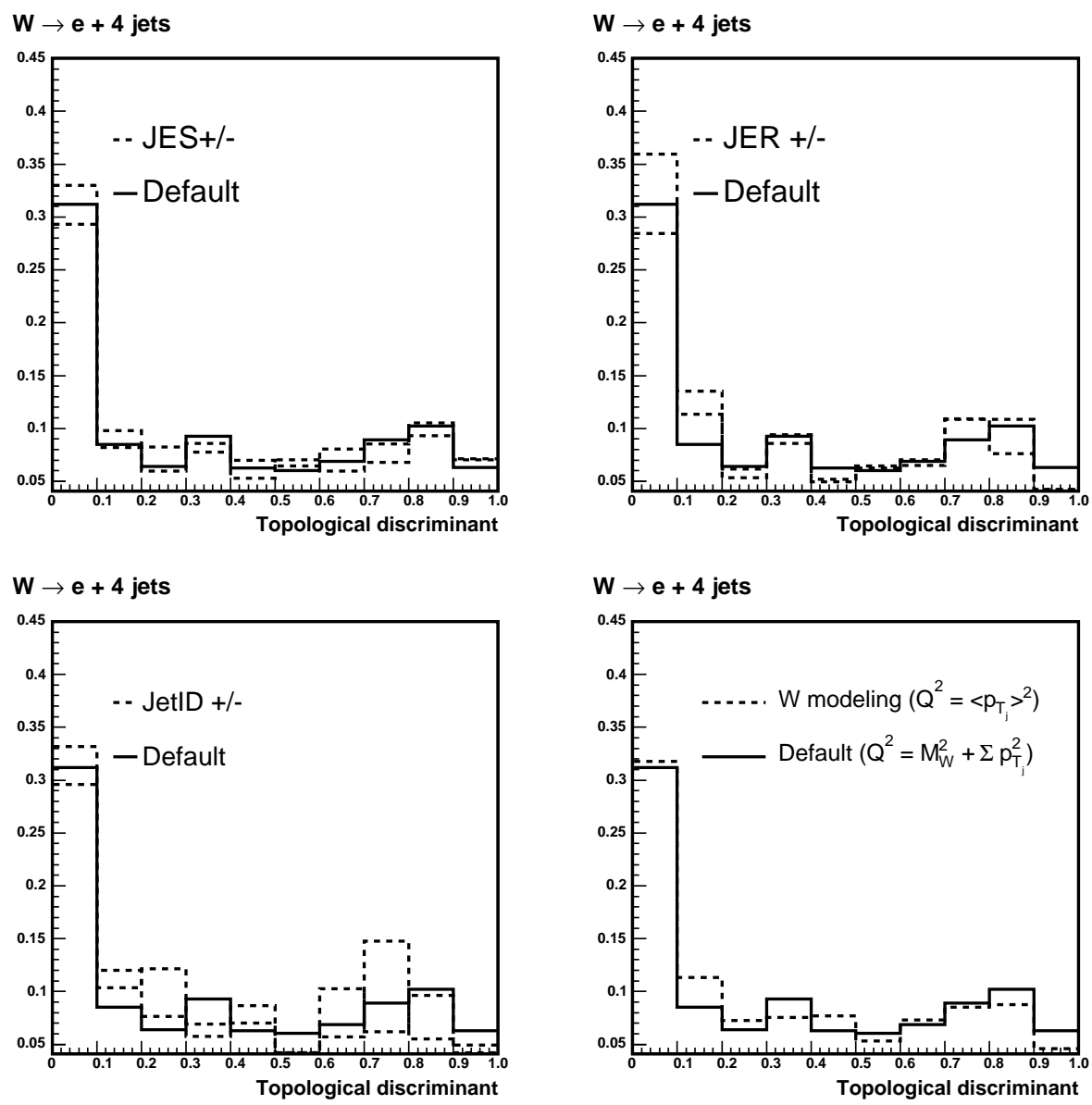

Figure 9.14: Topological likelihood templates in the $e+$ jet channel for $W+$ jets. The solid line shows the default template shape, superimposed on the template shapes obtained by varying jet energy scale (JES), jet energy resolution (JER), jet reconstruction and identification efficiency (JetID) and the factorization scale for the $W+$ jets sample ( $W$-modeling). Similar shifts are observed in the $\mu+$ jets channel. 


\subsubsection{Result}

The result of the simultaneous measurement of $\sigma_{t \bar{t}}$ and $R$ is obtained using an extended version of the nuisance parameter likelihood function in Eq. 9.9. The ratio $R$ is implemented as a free parameter in the likelihood and the constraint from the events without $b$-tags, given in Eq. 9.19, is added.

Using the new nuisance parameter likelihood function, the simultaneous measurement of $\sigma_{t \bar{t}}$ and $R$ in the $\ell+$ jets final state at $\sqrt{s}=1.96 \mathrm{TeV}$ yields:

$$
\begin{aligned}
R & \left.=1.03_{-0.17}^{+0.19} \text { (stat }+ \text { syst }\right) \\
\sigma_{t \bar{t}} & \left.=7.87_{-1.49}^{+1.67} \text { (stat }+ \text { syst }\right) \pm 0.51 \text { (lumi) } \mathrm{pb}
\end{aligned}
$$

The $68 \%$ and $95 \%$ C.L. contours are shown in Fig. 9.15. The list of systematic uncertainties is given in Tab. 9.8 for $R$ and in Tab. 9.9 for $\sigma_{t \bar{t}}$. The results for $\sigma_{t \bar{t}}$ and $R$ above are obtained for a top quark mass of $175 \mathrm{GeV} / c^{2}$. The value for the $t \bar{t}$ cross section is shown as function of the top quark mass in Fig. 9.16(a). A linear fit in the mass region $160-190 \mathrm{GeV} / c^{2}$ indicates that $\sigma_{t \bar{t}}$ varies by $0.04 \mathrm{pb}$ per $\mathrm{GeV} / c^{2}$ away from $175 \mathrm{GeV} / c^{2}$. The value of $R$ as a function of the top quark mass is shown in Fig. 9.16(b). $R$ decreases by 0.001 for each increase of $1 \mathrm{GeV} / c^{2}$ on the top quark mass.

If the experimental ratio $R$ is interpreted in terms of the standard model branching fractions in Eq. 9.1 the value of $R$ is restricted to be in the range $0 \leq R \leq 1$. Using a Bayesian approach with the prior

$$
\pi(R)=\left\{\begin{array}{lc}
1 & \text { if } 0 \leq R \leq 1 \\
0 & \text { if } R<0 \text { or } R>1,
\end{array}\right.
$$

a lower limit on $R$ can be derived. The resulting lower limits on $R$ are

$$
\begin{aligned}
& 68 \% \mathrm{CL}: \quad R>0.78 \\
& 95 \% \mathrm{CL}: \quad R>0.61
\end{aligned}
$$

which are illustrated in Fig. 9.17 (left plot). The lower limit on $\left|V_{t b}\right|$ can be extracted from $R$ assuming that $\left|V_{t b}\right|=\sqrt{R}$. The lower limits on $R$ implies the following lower limits on $\left|V_{t b}\right|$ :

$$
\begin{array}{ll}
68 \% \mathrm{CL}: & \left|V_{t b}\right|>0.88 \\
95 \% \mathrm{CL}: & \left|V_{t b}\right|>0.78
\end{array}
$$

The lower limits on $\left|V_{t b}\right|$ are illustrated in Fig. 9.17 (right plot). The measured value of $R=1.03_{-0.17}^{+0.19}$ is in excellent agreement with the standard model prediction of $R \approx 1$. The value of $\sigma_{t \bar{t}}$ is also in good agreement with the value obtained in the $t \bar{t}$ cross section measurement described in Sec. 9.1. 


\begin{tabular}{|l|c|c|cc|}
\hline Systematic source & $R$ & Offset & $\sigma^{+}$ & $\sigma^{-}$ \\
\hline \hline Statistical uncertainty only & 1.027 & - & +0.172 & -0.155 \\
\hline Preselections efficiencies, $e+$ jets & & $<0.001$ & +0.003 & -0.000 \\
Preselections efficiencies, $\mu+$ jets & & $<0.001$ & +0.000 & -0.000 \\
Electron trigger requirements & & $<0.001$ & +0.003 & -0.000 \\
Muon trigger requirements & & $<0.001$ & +0.000 & -0.000 \\
Jet trigger requirements & & $<0.001$ & +0.000 & -0.000 \\
Jet energy scale & & $<0.001$ & +0.015 & -0.011 \\
Jet energy resolution & & -0.003 & +0.034 & -0.027 \\
Jet reconstruction and identification & & $<0.001$ & +0.004 & -0.003 \\
Taggability & & +0.002 & +0.059 & -0.050 \\
Simulated SVT parameterizations & & $<0.001$ & +0.005 & -0.002 \\
b-tagging efficiency in data & & $<0.001$ & +0.000 & -0.008 \\
Mistag rate & & +0.001 & +0.004 & -0.004 \\
$\varepsilon_{\text {lept }}$ and $\varepsilon_{\text {instr }}$ in $e+$ jets channel & & $<0.002$ & +0.016 & -0.018 \\
$\varepsilon_{\text {lept }}$ and $\varepsilon_{\text {instr }}$ in $\mu+$ jets channel & & $<0.001$ & +0.003 & -0.002 \\
Event statistics for matrix method & & $<0.001$ & +0.022 & -0.013 \\
Limited statistics in simulated samples & & $<0.001$ & +0.012 & -0.009 \\
$W+$ jets flavor fractions, MLM comparison & & $<0.001$ & +0.044 & -0.041 \\
\hline Remaining unc. on $W+$ jets flavor fractions & & - & +0.091 & -0.077 \\
$W+$ jets likelihood template uncertainty & & +0.003 & +0.193 & -0.172 \\
\hline Total systematic uncertainty & & & -0.017 \\
\hline Total unc. (nuisance parameter likelihood) & 1.030 & + & \\
\hline
\end{tabular}

Table 9.8: Summary of the uncertainties on $R$ in Eq. 9.21, obtained using the nuisance parameter likelihood. This likelihood allows systematic uncertainties to shift the central value of $R$. The shift is shown in the column labeled "Offset". The columns labeled $\sigma^{+}$and $\sigma^{-}$show the contribution to the total uncertainty on $R$. 


\begin{tabular}{|l|c|c|cc|}
\hline Systematic source & $\sigma_{t \bar{t}}$ & Offset & $\sigma^{+}$ & $\sigma^{-}$ \\
\hline \hline Statistical uncertainty only & 7.97 & - & +1.42 & -1.27 \\
\hline Preselection efficiencies, $e+$ jets & & -0.02 & +0.18 & -0.14 \\
Preselection efficiencies, $\mu+$ jets & & +0.02 & +0.16 & -0.13 \\
Electron trigger requirements & & $<0.01$ & +0.03 & -0.03 \\
Muon trigger requirements & & +0.07 & +0.33 & -0.25 \\
Jet trigger requirements & & $<0.01$ & +0.00 & -0.01 \\
Jet energy scale & & -0.04 & +0.30 & -0.26 \\
Jet energy resolution & & $<0.01$ & +0.13 & -0.12 \\
Jet reconstruction and identification & & +0.02 & +0.15 & -0.16 \\
Taggability & & $<0.01$ & +0.00 & -0.01 \\
Simulated SVT parameterizations & & $<0.01$ & +0.03 & -0.05 \\
b-tagging efficiency in data & & $<0.01$ & +0.08 & -0.07 \\
Mistag rate & & $<0.01$ & +0.07 & -0.09 \\
$\varepsilon_{\text {lept }}$ and $\varepsilon_{\text {instr }}$ in $e+$ jets channel & & $<0.01$ & +0.13 & -0.00 \\
$\varepsilon_{\text {lept }}$ and $\varepsilon_{\text {instr }}$ in $\mu+$ jets channel & & $<0.01$ & +0.05 & -0.04 \\
Event statistics for matrix method & & -0.04 & +0.19 & -0.19 \\
Limited statistics in simulated samples & & $<0.01$ & +0.07 & -0.06 \\
$W+$ jets flavor fractions, MLM comparison & & -0.04 & +0.46 & -0.48 \\
Remaining unc. on $W+$ jets flavor fractions & & -0.02 & +0.24 & -0.23 \\
$W+$ jets likelihood template uncertainty & & $<0.01$ & +0.41 & -0.30 \\
\hline Total systematic uncertainty & & - & +0.90 & -0.80 \\
\hline Total uncertainty (nuisance par. likelihood) & 7.87 & -0.10 & +1.67 & -1.49 \\
\hline
\end{tabular}

Table 9.9: Summary of the uncertainties on the $t \bar{t}$ cross section in Eq. 9.22, obtained using the nuisance parameter likelihood. This likelihood allows systematic uncertainties to shift the central value of the $t \bar{t}$ cross section. The shift is shown in the column labeled "Offset". The columns labeled $\sigma^{+}$and $\sigma^{-}$show the contribution to the total uncertainty on the cross section. 


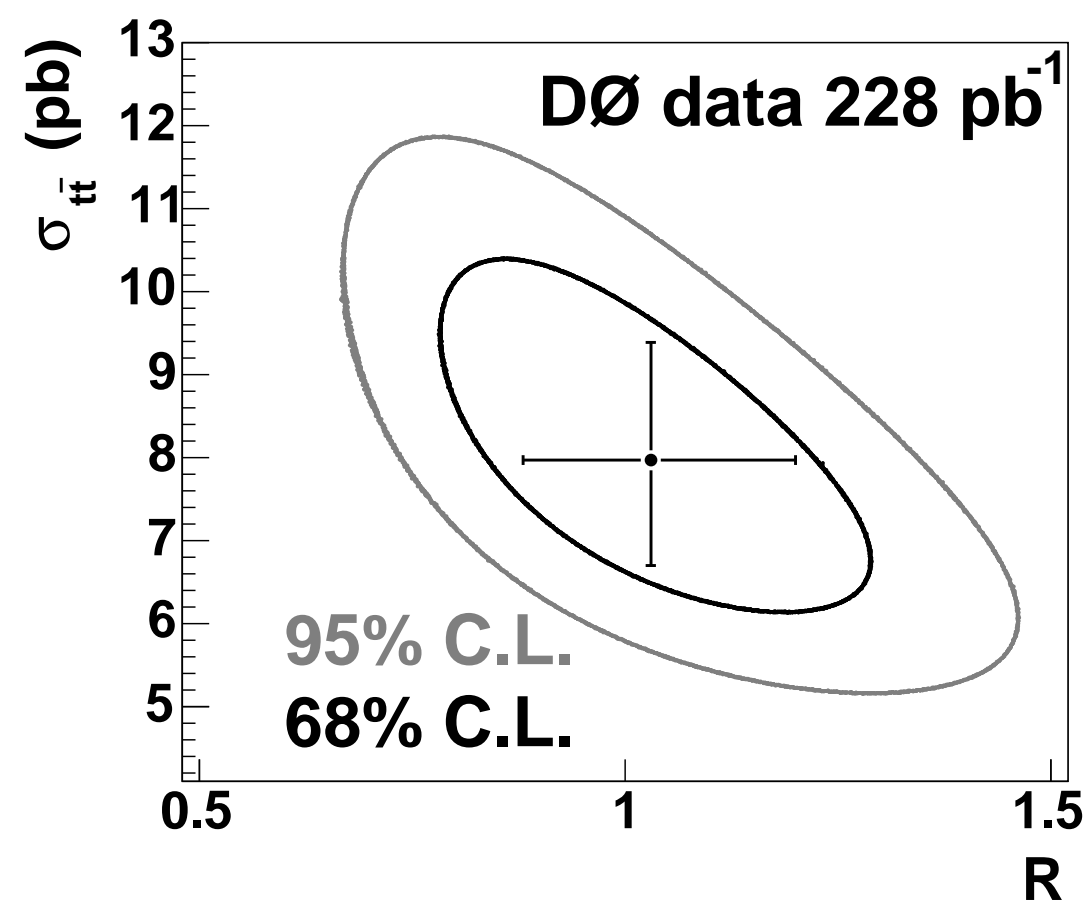

Figure 9.15: The $68 \%$ and $95 \%$ C.L. contours in the plane of $\left(R, \sigma_{t \bar{t}}\right)$. The point with error bars shows the measured values of $\sigma_{t \bar{t}}$ and $R$ with the statistical uncertainties.

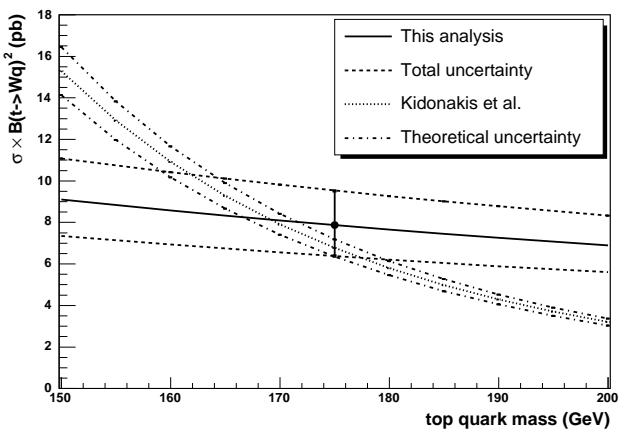

(a) $\sigma_{t \bar{t}}$ dependence on the top quark mass.

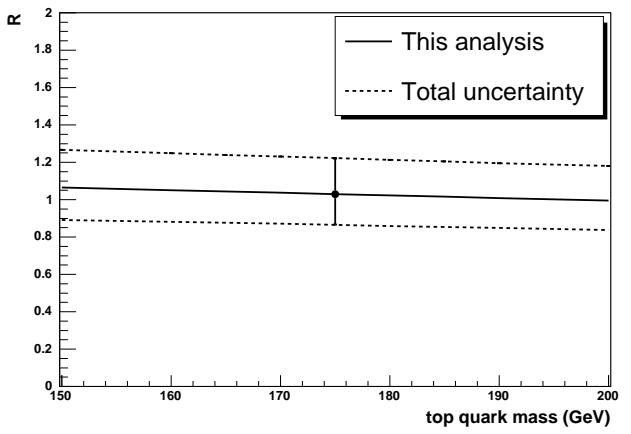

(b) $R$ dependence on the top quark mass.

Figure 9.16: The dependence of $\sigma_{t \bar{t}}$ and $R$ on the mass of the top quark. 

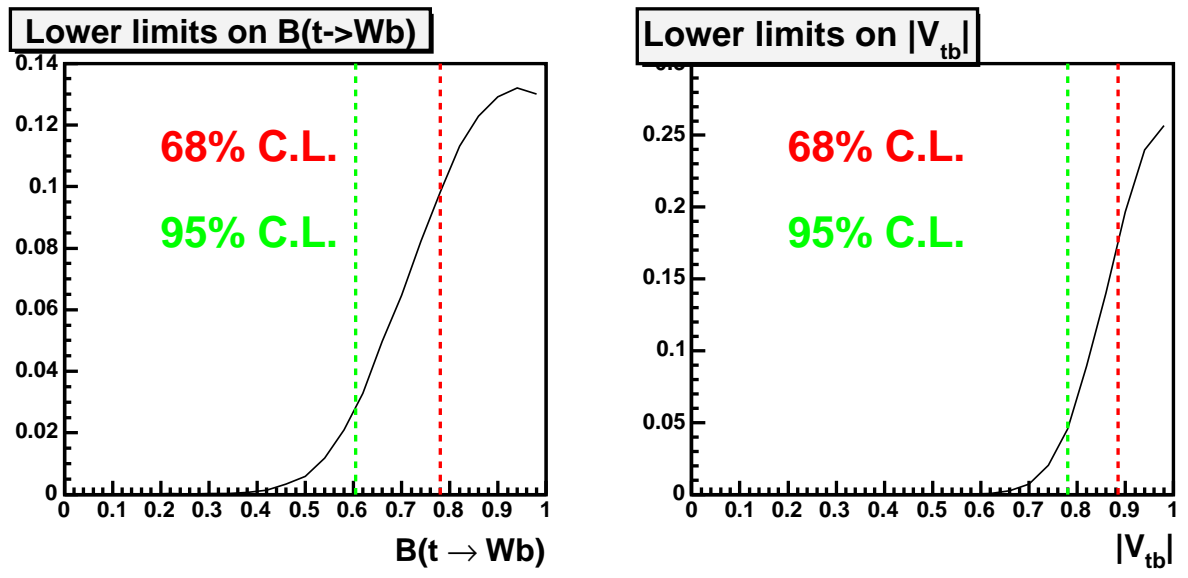

Figure 9.17: The $68 \%$ and 95\% C.L. lower limits on $R$ (left) and $\left|V_{t b}\right|$ (right). 


\section{Chapter 10}

\section{Conclusions and Outlook}

This thesis is based on the results of two papers. The first paper presents a measurement of the $t \bar{t}$ cross section in the $\ell+$ jets channel. The data sample used corresponds to an integrated luminosity of approximately $230 \mathrm{pb}^{-1}$. The second paper uses the same data sample to perform a simultaneous measurement of $\sigma_{t \bar{t}}$ and $R$.

Assuming the standard model decay of the top quark, the measured $t \bar{t}$ production cross section in the $\ell+$ jets final state at $\sqrt{s}=1.96 \mathrm{TeV}$ is:

$$
\left.\sigma_{t \bar{t}}=8.58_{-1.47}^{+1.64} \text { (stat }+ \text { syst }\right) \pm 0.55 \text { (lumi) pb. }
$$

This is in good agreement with the standard model prediction of $6.70_{-0.88}^{+0.71} \mathrm{pb}[26,27]$. When the result in Paper I was presented for the first time at the Winter conferences in 2005 [87], it was one of the most precise single measurements of the $t \bar{t}$ cross section. Figure 10.1 summarizes all the $\mathrm{D} \emptyset$ measurements of the $t \bar{t}$ cross section. The measurement labeled vertex tag $230 \mathrm{pb}^{-1}$ corresponds to the result presented in this thesis. This analysis was the first published top quark measurement from D $\varnothing$ using $b$-tagging [88].

The $t \bar{t}$ cross section measurement has been updated with a larger data sample. The latest cross section results from $\mathrm{D} \varnothing$ and CDF were presented at the LeptonPhoton conference in Uppsala in the Summer of 2005. The result labeled vertex tag $363 \mathrm{pb}^{-1}$ in Fig. 10.1 corresponds to the updated result from $\mathrm{D} \varnothing$ in the $\ell+$ jets channel using $b$-tagging. Figure 10.2 summarizes the results from the CDF collaboration. The measurement in the $\ell+$ jets channel using $b$-tagging continues to be one of the most precise results.

The simultaneous measurement of the ratio $R=B(t \rightarrow W b) / B(t \rightarrow W q)$ and $\sigma_{t \bar{t}}$ is performed using events with 0,1 and 2 secondary vertex tagged jets. It is the first measurement of $R$ in the $\mathrm{D} \varnothing$ collaboration. The result is:

$$
\begin{aligned}
R & =1.03_{-0.17}^{+0.19}(\text { stat }+ \text { syst }) \\
\sigma_{t \bar{t}} & =7.87_{-1.49}^{+1.67}(\text { stat }+ \text { syst }) \pm 0.51(\text { lumi }) \mathrm{pb}
\end{aligned}
$$




\section{Dø Run II Preliminary}

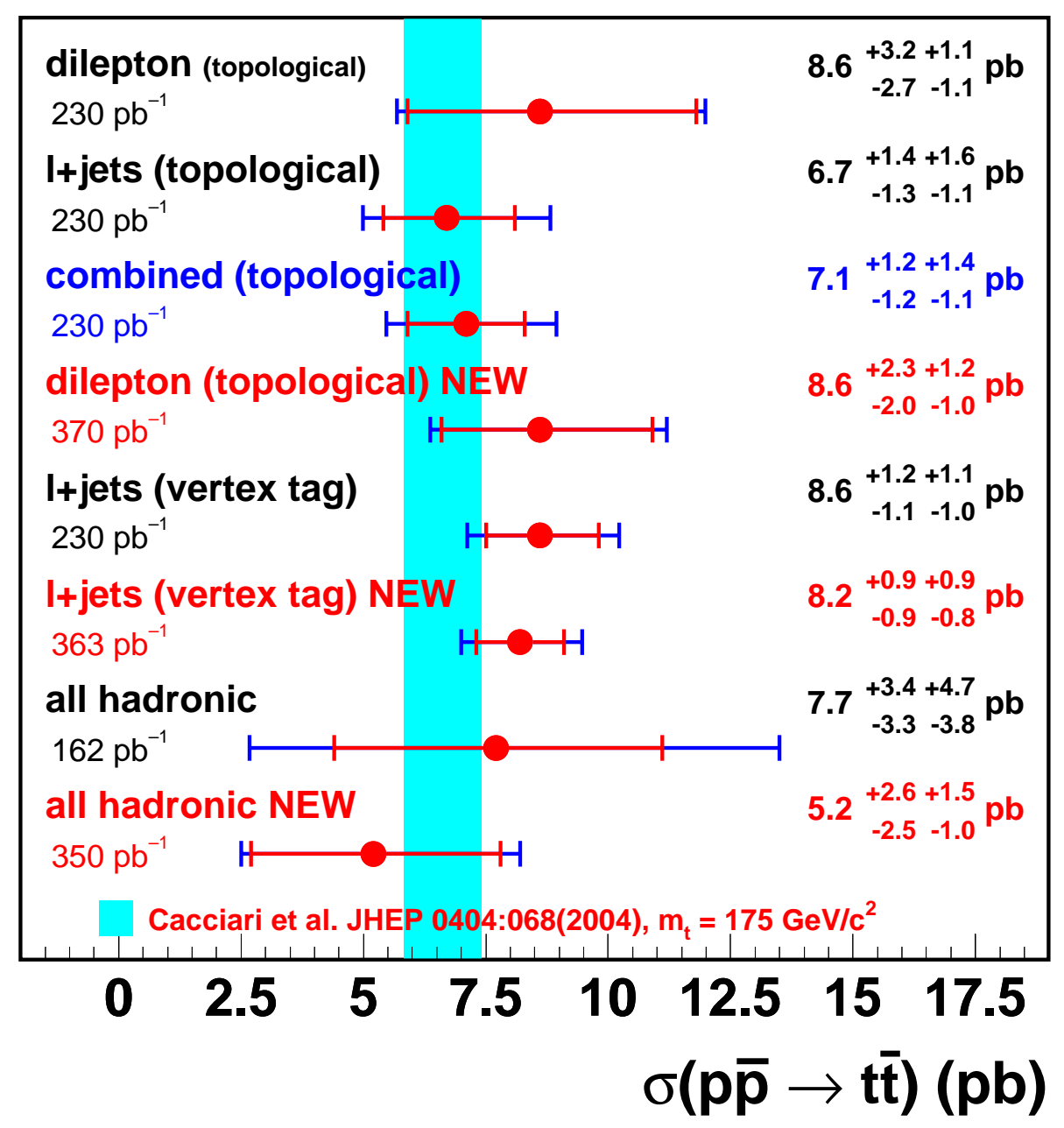

Figure 10.1: Summary of the $t \bar{t}$ cross section measurements performed by the $D \varnothing$ collaboration. The result labeled vertex tag $230 \mathrm{pb}^{-1}$ corresponds to the measurement presented in this thesis. The standard model prediction for the $t \bar{t}$ cross section is overlaid as the vertical band. 


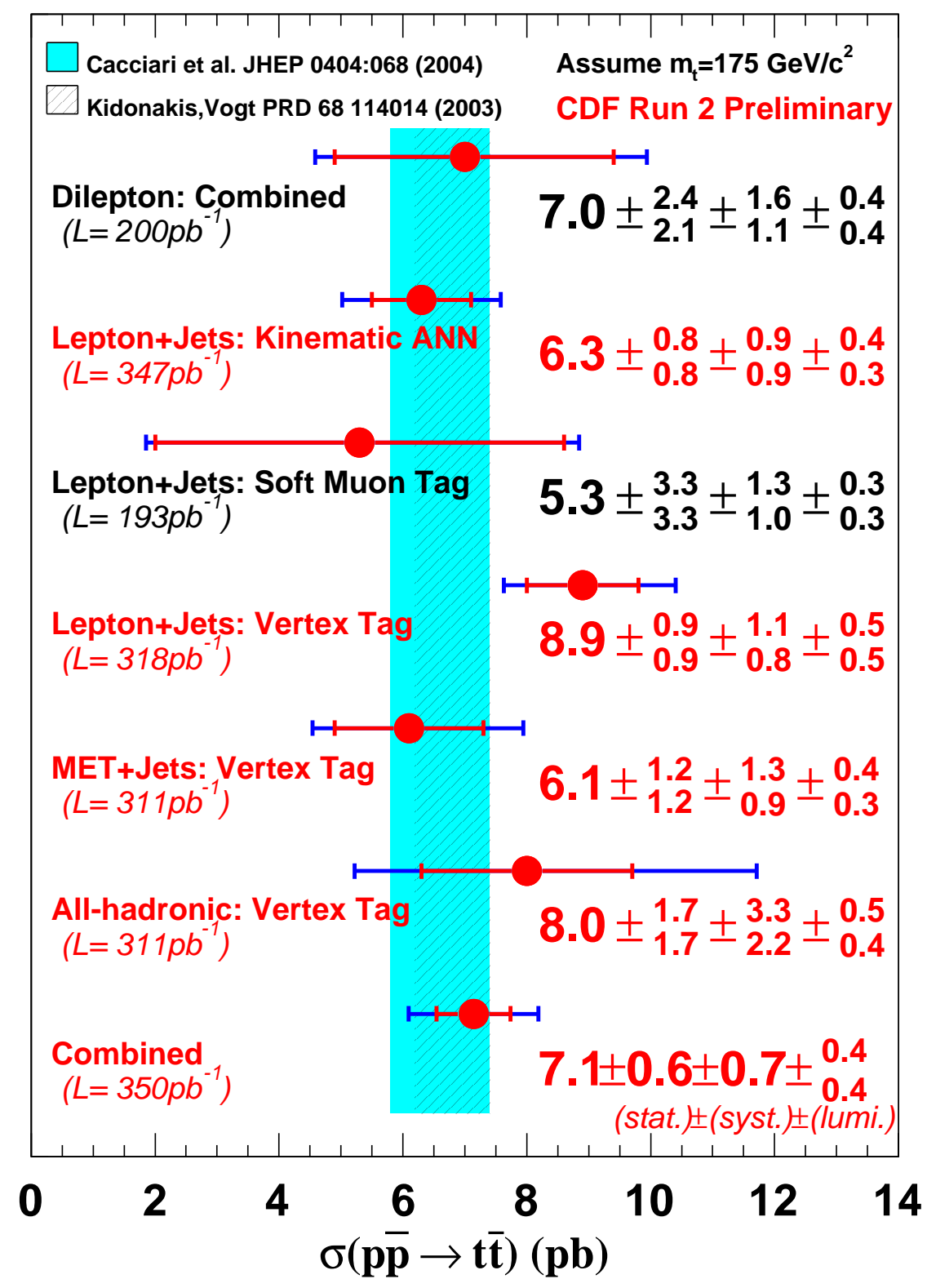

Figure 10.2: Summary of the $t \bar{t}$ cross section measurements presented at the LeptonPhoton conference by the CDF collaboration. The standard model prediction for the $t \bar{t}$ cross section is overlaid as the vertical band. 
A lower limit on $R$ is derived using a Bayesian approach:

$$
\begin{aligned}
& 68 \% \mathrm{CL}: \quad R>0.78 \\
& 95 \% \mathrm{CL}: \quad R>0.61
\end{aligned}
$$

The lower limit on $R$ can be transformed into a lower limit on the CKM matrix element $\left|V_{t b}\right|$,

$$
\begin{array}{ll}
68 \% \mathrm{CL}: & \left|V_{t b}\right|>0.88 \\
95 \% \mathrm{CL}: & \left|V_{t b}\right|>0.78
\end{array}
$$

assuming $\left|V_{t b}\right|=\sqrt{R}$. These results are in good agreement with the predictions from the standard model. CDF has performed a similar analysis of $R$ [89] with the result:

$$
R^{\mathrm{CDF}}=1.12_{-0.19}^{+0.21} \text { (stat) }{ }_{-0.13}^{+0.17} \text { (syst) }
$$

Single top quarks can also be produced via the weak force. This production mode has not yet been observed experimentally due to the large background. The sensitivity of the searches for single top quarks [90,91] should be sufficient for a discovery at the Tevatron in the near future. Measuring the single top quark production cross section will be a direct measurement of the CKM matrix element $\left|V_{t b}\right|$.

The Large Hadron Collider at CERN is projected to start data taking in the year 2007. The LHC will increase both the center-of-mass energy and the instantaneous luminosity by an order of magnitude compared to the Tevatron. More than one $t \bar{t}$ event per second will be produced. The top quark will continue to play a prominent role in the physics program at the LHC. Because of its large mass and intimate connection with the Higgs boson, the top quark could play an important role in the electro-weak symmetry breaking. The large sample of $t \bar{t}$ events will be important to calibrate the detectors during the early period of data taking. Using a sample of $t \bar{t} \rightarrow \ell+$ jets events, the jet energy scale can be calibrated from the invariant mass spectrum of the two jets from the hadronically decaying $W$ boson.

Above all the LHC is a machine to search for new physics beyond the standard model. Time will tell what type of new physics will be discovered, but any scenario where the signature involves high $p_{T}$ leptons, high $\not_{T}$ or several high $p_{T}$ jets will have $t \bar{t}$ events as one of the main backgrounds. Top quark physics therefore continues to be of great interest through 2007 and beyond. 


\section{Bibliography}

[1] D. J. Gross and F. Wilczek, Phys. Rev. D 8 (1973) 3633.

[2] H. D. Politzer, Phys. Rept. 14 (1974) 129.

[3] M. Gell-Mann, Phys. Lett. 8 (1964) 214-215.

[4] S. Weinberg, Phys. Rev. Lett. 19 (1967) 1264.

[5] S. L. Glashow, Nucl. Phys. 22 (1961) 579.

[6] A. Salam and J. C. Ward, Phys. Lett. 13 (1964) 168-171.

[7] F. Mandl and G. Shaw, Quantum field theory, Chichester, UK: Wiley, 1984.

[8] M. Chaichian and N. F. Nelipa, Introduction to Gauge Field Theories, SpringerVerlag Berlin Heidelberg, 1984.

[9] The LEP Collaborations and the LEP Electroweak Working Group, hep-ex/0511027 (2005).

[10] P. W. Higgs, Phys. Lett. 12 (1964) 132-133.

[11] F. Englert and R. Brout, Phys. Rev. Lett. 13 (1964) 321-322.

[12] G. S. Guralnik, C. R. Hagen and T. W. B. Kibble, Phys. Rev. Lett. 13 (1964) 585-587.

[13] Super-Kamiokande Collaboration, Phys. Rev. Lett. 93 (2004) 101801.

[14] SNO Collaboration, Phys. Rev. Lett. 87 (2001) 071301.

[15] A. D. Sakharov, Pisma Zh. Eksp. Teor. Fiz. 5 (1967) 32-35 and JETP Lett. 5 (1967) 24-27.

[16] DØ Collaboration, Phys. Rev. Lett. 74 (1995) 2632.

[17] CDF Collaboration, Phys. Rev. Lett. 74 (1995) 2626. 
[18] P. C. Bhat and W. J. Spaulding, hep-ex/0410046 (2005).

[19] DØ Collaboration, hep-physics/0507191 (2005).

[20] CDF Collaboration, Nucl. Instrum. Meth. A 462 (2001) 170-173.

[21] Paper in preparation. A preliminary version of the paper can be found at http://www-d0.fnal.gov/ asman/d0_private.

[22] S. Eidelman et al., Phys. Lett. B 592 (2004) 1.

[23] Aleph, Delphi, L3 and Opal Collaborations, Phys. Lett. B 565 (2003) 61-75.

[24] S. Catani, hep-ph/0005233 (2000).

[25] CTEQ Collaboration, Eur. Phys. J. C12 (2000) 375-392.

[26] R. Bonciani, S. Catani, M. Mangano, and P. Nason, Nucl. Phys. B 529 (1998) 424 .

[27] M. Cacciari, S. Frixione, G. Ridolfi, M. Mangano, P. Nason, JHEP 404 (2004) 68.

[28] N. Kidonakis and R. Vogt, Phys. Rev. D 68 (2003) 114014.

[29] N. Kidonakis and R. Vogt, Eur. Phys. J. C68 (2004) 466.

[30] N. Kidonakis, E. Laenen, S. Moch and R. Vogt, Phys. Rev. D 64 (2001) 114001.

[31] B. W. Harris et. al, Phys. Rev. D 66 (2002) 054024.

[32] Z. Sullivan, Phys. Rev. D 70 (2004) 114012.

[33] J. Campbell, R. K. Ellis and F. Tramontano, Phys. Rev. D 70 (2004) 094012.

[34] Q.-H. Cao, R. Schwienhorst and C.-P. Yuan, Phys. Rev. D 71 (2005) 054023.

[35] Q.-H. Cao et. al, Phys. Rev. D 72 (2005) 094027.

[36] A. Czarnecki and K. Melnikov, Nucl. Phys. B544 (1999) 520-531.

[37] The CDF Collaboration, the DØ Collaboration and the Tevatron Electroweak Working Group, hep-ex/0507091 (2005).

[38] DØ Collaboration, Phys. Rev. D 58 (1998) 052001.

[39] CDF Collaboration, Phys. Rev. D 63 (2001) 032003.

[40] DØ Collaboration, Nature 429 (2004) 638. 
[41] CDF Collaboration, CDF/ANAL/TOP/PUB/7239 (2004).

CDF Collaboration, CDF Note 7194 (2004).

CDF Collaboration, CDF/PHYS/TOP/PUBLIC/7303 (2005).

[42] CDF Collaboration, CDF/PHYS/TOP/PUBLIC/7102 (2004).

CDF Collaboration, CDF/PHYS/TOP/PUBLIC/7056 (2004).

CDF Collaboration, CDF Note 7637 (2005).

[43] CDF Collaboration, CDF/ANAL/TOP/PUB/7680 (2005).

[44] CDF Collaboration, CDF/ANAL/TOP/PUB/7718 (2005).

[45] DØ Collaboration, D $\varnothing$ Note 4725-CONF (2005).

[46] DØ Collaboration, DØ Note 4728-CONF (2005).

DØ Collaboration, DØ Note 4574-CONF (2004).

[47] DØ Collaboration, D $\varnothing$ Note 4874-CONF (2005).

[48] A. Castro, for the CDF and DØ Collaborations, FERMILAB-CONF-01-095-E (2001).

[49] The ATLAS Collaboration, CERN-LHCC-99-14 (1999).

[50] The ATLAS Collaboration, CERN-LHCC-99-15 (1999).

[51] http://d0server1.fnal.gov/users/stefan/www/CFT_TDR/CFT_TDR.ps

[52] R. Yarema et al., FERMILAB-TM-1892 (1994, revised 1996).

[53] DØ Collaboration, Nucl. Instrum. Meth. A 324 (1993) 53, FERMILAB-PUB-92/162-E.

[54] Private communication with detector experts.

[55] V. M. Abazov et al., Nucl. Instrum. Meth. A 552 (2005) 372-398.

[56] T. Edwards et al., FERMILAB-TM-2278-E (2004).

[57] M. Narain and F. Stichelbaut, DØ Note 3560 (1998).

[58] A. Garcia-Bellido et al., DØ Note 4320 (2004).

[59] A. Schwartzman and M. Narain, DØ Note 4042 (2002).

[60] J. Zhu and V. Buscher, DØ Note 4171 (2003).

[61] J. Kozminski et al., DØ Note 4449 (2004). 
[62] C. Clément et al., DØ Note 4350 (2004).

[63] G. Blazey et al., DØ Note 3750 (2000).

[64] E. Busato and B. Andrieu, DØ Note 4457 (2004).

[65] DØ Jet Energy Scale group, http://www-d0.fnal.gov/phys_id/jes/d0_private/certified/v5.1/links.html

[66] R. Brun and F. Carminati, CERN Program Library Long Writeup W5013 (1993).

[67] M.L. Mangano et al., JHEP 0307 (2003) 001, hep-ph/0206293.

[68] T. Sjöstrand et al., Comput. Phys. Commun. 135 (2001) 238.

[69] R. Fields, Min-Bias and the Underlying Event at the Tevatron and the LHC, talk presented at the Fermilab ME/MC Tuning Workshop, Fermilab, Oct 4, 2002 .

[70] CDF Collaboration, Phys. Rev. D 65 (2002) 092002.

[71] D. Lange, Nucl. Instrum. Meth. A 462 (2001) 152.

[72] S. Jadach et al., Comput. Phys. Commun. 76 (1993) 361.

[73] S. Catani et al., JHEP 0111 (2001) 063.

[74] F. Krauss, JHEP 0208 (2002) 015.

[75] S. Höche et al., hep-ph/0602031 (2006).

[76] J.M. Campbell and R.K. Ellis, Phys. Rev. D 62 (2000) 114012.

[77] J.M. Campbell and R.K. Ellis, Phys. Rev. D 65 (2002) 113007.

[78] J.M. Campbell and J. Huston, submitted to Phys. Rev. D, hep-ph/0405276.

[79] J.M. Campbell and R.K. Ellis, Phys. Rev. D 60 (1999) 113006.

[80] T. Nunnemann, DØ Note 4476 (2004).

[81] CompHEP Collaboration, Nucl. Instrum. Meth. A 534 (2004) 250.

[82] C. Clément et al., DØ Note 4662 (2004).

[83] T. Golling, DØ Note 4667 (2004).

[84] A. Schwartzman and M. Narain, DØ Note 3908 (2001). 
[85] B. Clément et al., DØ Note 4159 (2003).

[86] E. T. Jaynes, Probability Theory, University Press, Cambridge 2003.

[87] DØ Collaboration, hep-ex/0511034 (2005).

[88] DØ Collaboration, Phys. Lett. B 626 (2005) 35.

[89] CDF Collaboration, Phys. Rev. Lett. 95 (2005) 102002.

[90] DØ Collaboration, Phys. Lett. B 622 (2005) 265-276.

[91] CDF Collaboration, Mod. Phys. Lett. A 19 (2004) 1493-1507. 